INEL-96/0375

January 1997

\title{
Performance Assessment for a Hypothetical Low-Level Waste Disposal Facility
}

Cary S. Smith

Michael J. Rohe

Paul D. Ritter

Craig A. Dicke

Arthur S. Rood

Russell S. Garcia

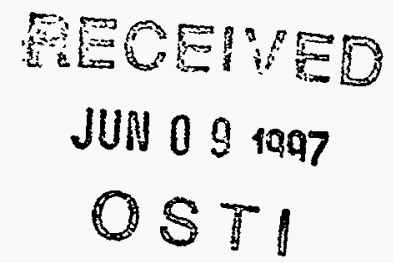




\title{
Performance Assessment for a Hypothetical Low-Level Waste Disposal Facility
}

\author{
Cary S. Smith \\ Michael J. Rohe \\ Paul D. Ritter \\ Craig A. Dicke \\ Arthur S. Rood \\ Russell S. Garcia
}

Published January 1997

\begin{abstract}
Idaho National Engineering Laboratory Lockheed Martin Idaho Technologies Integrated Earth Sciences Department Idaho Falls, Idaho 83415
\end{abstract}

Prepared for the U.S. Department of Energy

Assistant Secretary for Environmental Management Under DOE Idaho Operations Office

Contract DE-AC07-94ID13223 


\section{DISCLAMMER}

Portions of this document may be illegible in electronic image products. Images are produced from the best avaliable original document. 


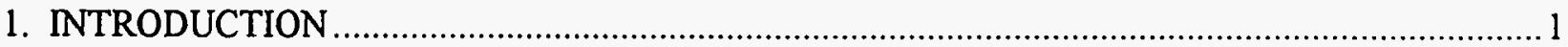

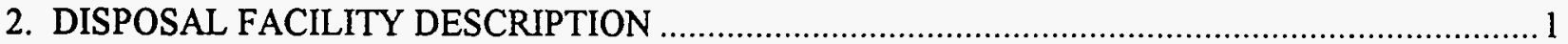

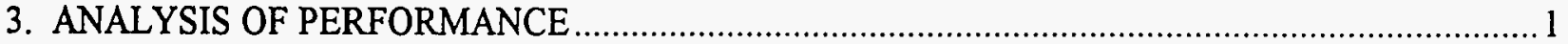

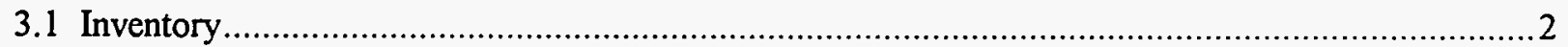

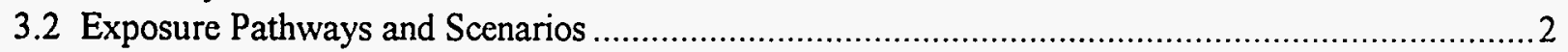

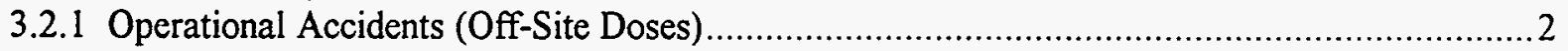

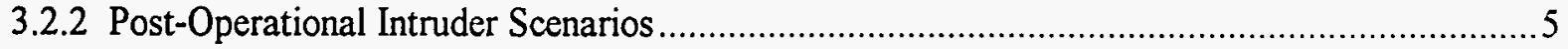

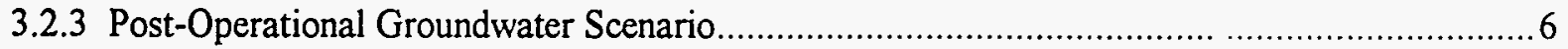

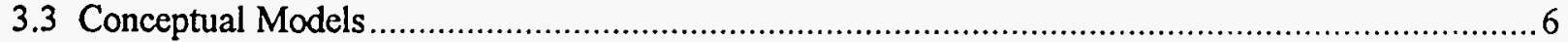

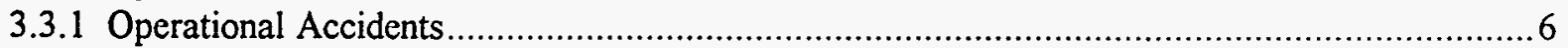

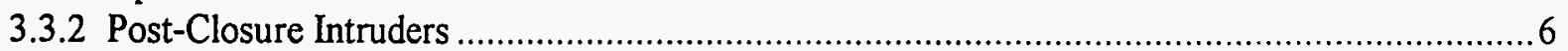

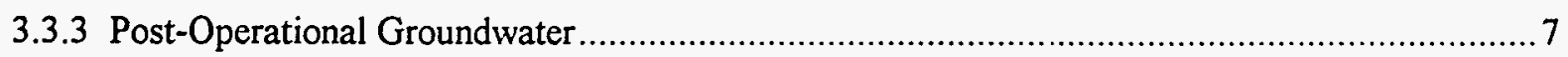

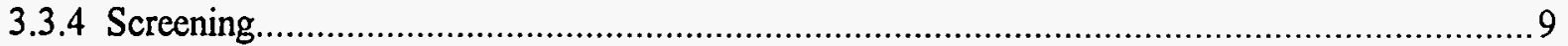

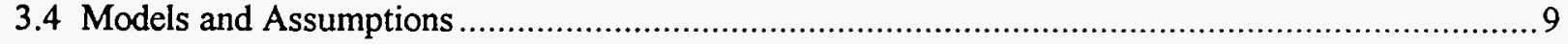

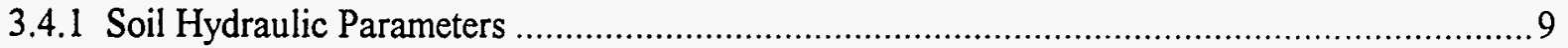

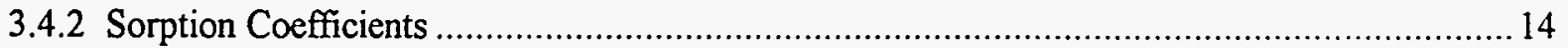

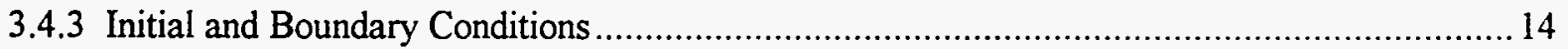

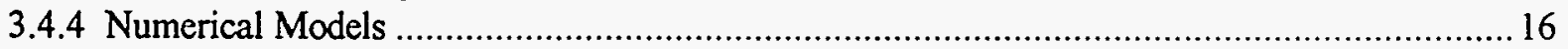

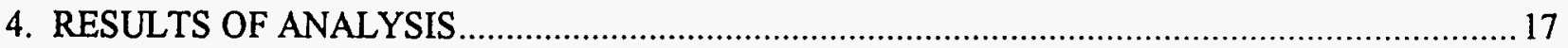

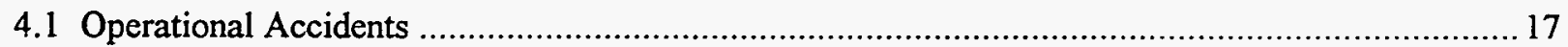

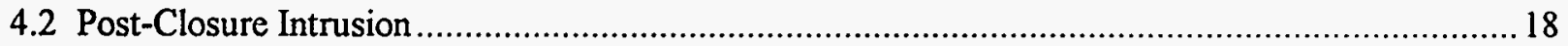

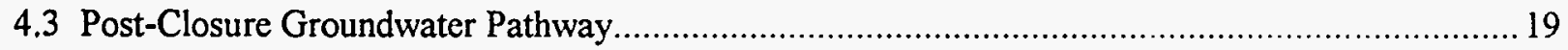

6. REFERENCES

Appendix $\mathrm{A}-$ Best Estimate Inventory for Class $\mathrm{AB}$ and $\mathrm{C}$ Waste ..................................................

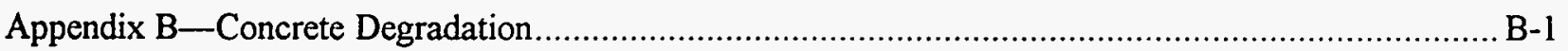

Appendix C-Engineering Design File for Sorption Coefficients ....................................................... C-1

Appendix D—GWSCREEN Model Simulation Output..................................................................... 


\section{FIGURES}

Figure 1. Conceptual model of water movement through the facility.

\section{TABLES}

1. Off-site exposure pathways for operational accidents at the Pennsylvania LLW facility ......................3

2. Hydraulic parameter sources for simulation of unsaturated zone performance analysis .................... 10

3. Hydraulic parameter values for each material type ............................................................... 11

4. Sorption coefficients for loam, concrete, and grouted waste ...................................................... 13

5. Input parameters for the GWSCREEN screening simulations...................................................15

6. Off-site doses (mrem) caused by bounding hypothetical operational accidents at the Pennsylvania

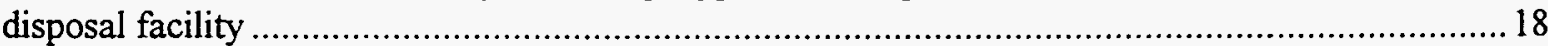

7. Doses (mrem) to individuals intruding into the Pennsylvania disposal facility ................................ 18

8. Percentage of dose from each intruder pathway for Class $A B$ and $C$ waste .................................. 19

9. Maximum committed effective dose equivalents calculated with GWSCREEN for Class AB waste.....19

10. Maximum committed effective dose equivalents calculated with GWSCREEN for Class $C$ waste. ....22

11. Maximum gross alpha activity in groundwater (excluding radium and radon) for Class $A B$ waste using the GWSCREEN code and screening model assumptions and methodology.................................24

12. Maximum gross alpha activity in groundwater (excluding radium and radon) for Class $C$ waste using the GWSCREEN code and screening model assumptions and methodology................................25

13. Initial and best estimate inventory, peak times, and maximum drinking water doses for ${ }^{234} \mathrm{U}$ and ${ }^{14} \mathrm{C}$, Class $\mathrm{AB}$ and $\mathrm{C}$ waste. 


\section{Performance Assessment for a Hypothetical Low-Level Waste Disposal Facility}

\section{INTRODUCTION}

Disposing of low-level waste (LLW) is a concern for many states throughout the United States. A common disposal method is below-grade concrete vaults. Performance assessment analyses make predictions of contaminant release, transport, ingestion, inhalation, or other routes of exposure, and the resulting doses for various disposal methods such as the below-grade concrete vaults. Numerous assumptions are required to simplify the processes associated with the disposal facility to make predictions feasible. In general, these assumptions are made conservatively so as to underestimate the performance of the facility.

The objective of this report is to describe the methodology used in conducting a performance assessment for a hypothetical waste facility located in the northeastern United States using real data as much as possible. This report consists of the following: (a) a description of the disposal facility and site, (b) methods used to analyze performance of the facility, (c) the results of the analysis, and (d) the conclusions of this study.

\section{DISPOSAL FACILITY DESCRIPTION}

The hypothetical LLW site was assumed to be located in the northeastern United States. The exact location was not chosen for political sensitivities. However, meteorological data necessary for the analysis were taken from Harrisburg, Pennsylvania. The facility was designed to contain class A, $\mathrm{B}$, and $\mathrm{C}$ wastes.

The design consists of two types of modular disposal units, which will be constructed of reinforced concrete. The larger vault $(122 \mathrm{~m}$ by $610 \mathrm{~m}$ by $7.6 \mathrm{~m}$ thick) will contain class $\mathrm{A}$ and $\mathrm{B}$ waste, while the smaller vault ( $24 \mathrm{~m}$ by $49 \mathrm{~m}$ by $3 \mathrm{~m}$ thick) will contain class $C$ waste. These units will consist of a base pad, supporting walls, and a sloping roof. The roof will be sloped to promote drainage of any water from the top of the modules. Upon closure, the units will be collectively covered with an engineered barrier intended to function throughout the period of institutional control (a 100year period following the end of the facility operational period).

The engineered earth cover will consist of several layers designed to minimize water infiltration and intrusion of biota down to the concrete modules. Infiltrating water will be diverted by encouraging evapotranspiration and redirecting water away from the disposal units.

\section{ANALYSIS OF PERFORMANCE}

This section describes the methodology used to evaluate the long-term performance of the facility. Each model component is described in this section of the report. These components are the inventory, exposure pathways, flow and transport models, and model simulations and assumptions. The source term describes the expected waste stream from generators, divided into two different classes of commercial LLW: class A and B wastes and class $\mathrm{C}$ wastes. Following the source term description is a detailed explanation of each pathway considered. The final two sections describe the conceptual and numerical models chosen to estimate the doses for each pathway. 


\subsection{Inventory}

The inventory of disposed radionuclides was provided by W. P. Dornsife, of the Pennsylvania Department of Environmental Protection on May 20, 1996 (Appendix A). Representative annual average inventories were derived from the combined inventories of expected waste shipments from a variety of commercial generators for an average year. This was then multiplied by the operating period for the facility, 30 years, to achieve the total inventory considered in this analysis.

\subsection{Exposure Pathways and Scenarios}

The exposure pathways considered in this analysis were external exposure, inhalation, ingestion, and airborne immersion. These exposure pathways were applied to different exposure scenarios depending on the assumptions used to generate the scenario. The scenarios considered were the following:

1. Exposure to members of the public through accidental release during operations (operational accidents).

2. Intrusion by a member of the public at the end of institutional control (post-operational intruder).

3. Groundwater ingestion by a member of the public living downgradient from the facility (postoperational groundwater).

The first exposure scenario was considered to occur only during the operational period of the facility. The operational period of the facility starts at the first shipment of waste and continues thereafter for 30 years. This exposure scenario considered exposure to the public through accidental releases only and evaluated the inhalation, external exposure, and air immersion exposure pathways.

The second exposure scenario considered doses to an individual member of the public who inadvertently intrudes into the closed facility following the facility's operational and institutional control period. Inhalation, external exposure, and ingestion exposure pathways are considered.

The third exposure scenario considered doses to the public that results from consumption of contaminated groundwater derived from a well located downgrading from the disposal the facility. Ingestion is the only exposure pathway included in this scenario. Each of these exposure scenarios are described in detail in the next sections.

\subsubsection{Operational Accidents (Off-Site Doses)}

Operational accident scenarios originally developed for the North Carolina disposal facility license application were used to estimate potential doses caused by accidents at the Pennsylvania facility. The design and planned operations for these disposal facilities are assumed to be similar, so adjustments to the source/receptor distances represented most of the adaptation of the North Carolina design basis accident scenarios to the Pennsylvania case. The sequence of events occurring in accidents initiated by natural events (e.g., earthquakes and tornadoes) is also assumed to be the samein particular, the exposure of individuals to released radioactive materials or penetrating radiation. The pathways by which individuals located off the Pennsylvania disposal facility site might be exposed are summarized in Table 1. Descriptions of the accident scenarios are provided in Sections 3.2.1.1 through 3.2.1.4. 
3.2.1.1 Fire in the Waste Packaging Building A number of human actions and natural events could cause a fire in the Waste Packaging Building. If a fire occurred, as-received, ungrouted, unsolidified waste would be more significantly affected than the stabilized waste packages. To bound these accidents, it is assumed that a liner of dewatered resin is exposed to a fire caused by ignition of the truck tractor fuel tanks. It is assumed that the fire lasts 30 minutes, which corresponds to a Severity II accident with a release fraction of 0.01 (Transportation EIS-NRC, 1977). Although dewatered resins consist primarily of millimeter diameter beads with a small quantity of crushed material (called fines), an aerosol fraction of 0.1 corresponding to fine powder is assumed (Neuhauser and Knipe, 1992). Per the North Carolina Safety Analysis Report (SAR), ${ }^{1}$ the product of the release fraction and aerosol fraction, 0.001 , corresponds to the flammability factor (as described in Oztunali and Roles, 1986). Radionuclide-specific release fractions (Oztunali and Roles, 1986) for materials involved in fires are also applied to determine the amount of radioactive material released to the air.

The fire is assumed to destroy the high-efficiency particulate air filters servicing the Waste Packaging Building and result in the release of a contaminated plume. An earlier analysis of a similar disposal site showed that the inventory of radioactive material already present on the high-efficiency particulate air filters at the time of the accident would be small when compared to that in the waste and can be ignored (CNSI, 1990). The release point is a vent on the roof of the building and is treated as a ground level release. Building wake effects were not considered, resulting in lower dispersion and a more conservative dose estimate. The minimum distance from the packaging building to the facility boundary is $1,600 \mathrm{ft}$, and an individual is assumed to remain at this position for 10 minutes during plume passage. Atmospheric stability and winds are assumed to be class $F$ and $2 \mathrm{~m} / \mathrm{s}$ during the fire, resulting in relatively little dilution of the released radioactive material.

Table 1. Off-site exposure pathways for operational accidents at the Pennsylvania LLW facility.

\begin{tabular}{lccc}
\multicolumn{1}{c}{ Scenario } & Inhalation & Immersion & Direct radiation ${ }^{2}$ \\
\hline $\begin{array}{l}\text { Fire in waste packaging } \\
\text { building }\end{array}$ & Yes & Yes & No \\
$\begin{array}{l}\text { Crane malfunction } \\
\text { suspended container }\end{array}$ & No & No & Yes \\
$\begin{array}{l}\text { Crane malfunction -- } \\
\text { dropped container }\end{array}$ & No & No & Yes \\
$\begin{array}{l}\text { Tornado } \\
\text { Earthquake }\end{array}$ & No & No & Yes \\
\hline $\begin{array}{l}\text { a. Direct radiation is penetrating radiation (photons) originating from materials contained within the } \\
\text { Pennsylvania Low-Level Waste Disposal facility buildings or intact waste containers. }\end{array}$ & Yes \\
\hline
\end{tabular}

3.2.1.1. Crane Malfunction. Two overhead bridge cranes will be used during Facility operations. A 40-ton overhead bridge crane in the Waste Packaging Building will be used to (a) off-

1. Personal Communication from Don Ballman to Paul Ritter, "Transmittal of Accident Scenario Information from the North Carolina License Application (referred to as the North Carolina SAR), December 28, 1995. 
load liners and some drums and boxes and (b) place the waste containers inside overpacks prior to grouting. A 50-ton overhead bridge crane will be used in the Movable Building to place the stabilized and better shielded waste packages into the disposal modules. Contact exposure rates on waste packages will be limited to 1 roentgen per hour, and the structural integrity of the waste packages is sufficient to sustain any possible drop with little chance of releases. Accordingly, the consequences of crane malfunctions in the Movable Building are less severe than those in the Waste Packaging Building, and only accidents in the Waste Packaging Building were considered. A failure of Waste Packaging Building overhead bridge crane during transfers could result in a radiation source being suspended above work areas in the Waste Packaging Building or cause waste containers to be dropped to the floor.

Liners with contact exposure rates of 20 roentgen per hour or more will be lifted with the liner inside a shield bell for transfer to a shielded overpack. Liners with contact exposure rates of more than 500 roentgen per hour will not be accepted at the Facility without special review and are outside the scope of this evaluation.

3.2.1.2.1 Container Suspended from the Overhead Bridge Crane A container suspended from the overhead crane could cause exposure of the public to gamma radiation. It is assumed that the shield bell containing a 500-roentgen-per-hour source is suspended $16 \mathrm{ft}$ above the Waste Packaging Building floor. This source was modeled in the MicroShield computer program (Grove Engineering, Inc., 1990; 1992) as a cylindrical source with a 3-in.-thick cylindrical iron shield to represent the shield bell. A member of the public is assumed to stand along the restricted area fence $1,600 \mathrm{ft}$ away from the source for 15 minutes.

The North Carolina SAR scenario involves a $97-\mathrm{ft}^{3}$ resin liner generating a $500-\mathrm{r} / \mathrm{h}$ radiation field near contact (i.e., at $10 \mathrm{~cm}$ from the midline on the side of the container). For this performance assessment, the liner was assumed to be cylindrical with the height equal to its diameter. The liner was assumed to contain radionuclides in the proportions in Table 6-28 of the North Carolina LLW Facility SAR, with total activity sufficient to generate a $500-\mathrm{r} / \mathrm{h}$ field at $10 \mathrm{~cm}$ from the surface of the liner. The liner was also modeled assuming the presence of ${ }^{60} \mathrm{Co}$ only, at the activity required to generate 500 $\mathrm{r} / \mathrm{h}$ at $10 \mathrm{~cm}$. The results for the latter case were somewhat greater and are reported in Section 4.1.

3.2.1.2.2 Container Dropped from the Overhead Crane The dropped container bounds accidents involving rupture of containers after receipt at the Facility in situations where only direct radiation exposure occurs, i.e., where there is no release of radioactivity from a building. The dropped container results in loss of the shielding provided by the 3-in.-thick iron shield bell described in Section 3.2.1.2.1. A container could be ruptured because of a drop initiated by (a) the same events that result in a suspended container, (b) a plane crashing into the Waste Packaging Building, or (c) a tornado. Since the greatest force would be generated by a drop from the crane to the floor, this scenario bounds accidents involving container rupture.

It is assumed that a member of the public stands in the unrestricted area 1,600 $\mathrm{ft}$ from the liner for 15 minutes, which is the length of time required to place temporary shielding. The high-efficiency particulati air filters prevent release of airborne contamination from the building. No credit is taken for the shielding provided by the building in estimating dose to the individual standing $1,600 \mathrm{ft}$ away in the unrestricted area.

3.2.1.3 Tornado. Tornados could cause considerable damage to buildings at the Facility. They may also generate projectiles that could damage waste containers or waste packages causing the 
release of radioactive material. Respirable radioactive material released during a tornado would be very rapidly dispersed, so the effects of container damage caused by wind-driven missiles are bounded by the dropped container accident. After the storm has passed, a member of the public or rescue worker could approach a container thrown into the unrestricted area and be exposed to direct radiation before facility workers could locate and retrieve the container. For the Pennsylvania LLW Performance Assessment, a tornado is assumed to destroy the Waste Packaging Building and throw a hypothetical drum of dry active waste, described in Table 6-29 of the North Carolina LLW SAR, into the unrestricted area where it is examined at a distance of $1 \mathrm{~m}$ for 30 seconds by a member of the general public. The exposure rate is 512 milliroentgen per hour, resulting in a dose of 4.3 millirem.

3.2.1.4 Earthquake. Earthquakes could also cause the overhead bridge cranes to malfunction, which could result in suspension or dropping of one or more waste contairiers or waste packages. Collapse of an overhead bridge crane or the concrete walls of the Waste Packaging Building could crush waste containers and result in release of radioactive material. Containers could also be shaken off trucks and rupture, releasing radioactive material. It is very unlikely that these events would cause release of radioactive material from the better-stabilized waste packages.

An earthquake with a ground acceleration in excess of the facility design specifications is assumed to occur and destroy the Waste Packaging Building. The overhead bridge crane collapses, crushing a group of 14 hypothetical drums of dry active waste that have been removed from a shipping cask.

\subsubsection{Post-Operational Intruder Scenarios}

3.2.2.1 End of the Institutional Control Period. An individual was assumed to construct a house above a disposal module at the end of the institutional control period (100 years post-closure). The roof of the module was assumed to be intact and prevent the intruder from excavating into waste. The intruder was assumed to use the roof of the module as the floor of the foundation for the house, and to use the basement of the house for routine household activities and for bedrooms. The module was assumed to contain the typical inventory of waste radionuclides at the time of closure. The intruder would receive a dose caused by penetrating radiation fields (gamma and $\mathrm{x}$-ray photons) emitted by the 100-year decayed inventory of the module.

3.2.2.2 500 Years After the End of Institutional Control. An individual was assumed to construct a house above a disposal module 500 years after the end of operations. The concrete roof of the madule was assumed to resemble soil. The intruder excavates a vertical-sided 10-ft-deep basement hole, removing the former roof material $(61 \mathrm{~cm}$ thick) and a $0.3-\mathrm{m}$ thick layer of waste from an area $10 \mathrm{~m}$ by $10 \mathrm{~m}$. The intruder spreads the waste and soil over the $2200 \mathrm{~m}^{2}$ yard that surrounds the house. The intruder raises vegetables grown in the contaminated soil and consumes meat from animals given fodder grown on the contaminated soil. The intruder receives doses caused by penetrating radiation fields (gamma and $\mathrm{x}$-ray photons) emitted by the undisturbed portion of the 500 year decayed inventory of the module (below and surrounding the lower part of the foundation) and the disturbed portion of this inventory (present in the top $15 \mathrm{~cm}$ of the soil in the yard). The intruder also receives doses because of consumption of contaminated home-grown foods and inadvertent ingestion of contaminated soil.

The disposal modules are designed to withstand the maximum credible earthquake. After the end of the institutional control period, the eventual consequences of damage from a severe earthquake are bounded by the assumptions made in estimating the source term caused by excavation. 


\subsubsection{Post-Operational Groundwater Scenario}

For the post-operational groundwater scenario, the receptor is assumed to reside at the waste buffer zone which is $100 \mathrm{~m}$ from the downgradient edge of the waste vaults. The receptor is assumed to have drilled a well at that location and used the water derived from that well as their primary source of water. The well is screened from the surface of the aquifer down to $15 \mathrm{~m}$. The receptor is assumed to drink $2 \mathrm{~L}$ of water a day for 365 days per year. Exposure is achieved by consumption of well water containing dissolved radionuclides.

\subsection{Conceptual Models}

Conceptual models are used to describe model compartments, flow paths, transport pathways, sources, and sinks, or may be simply a verbal description of the system to be modeled. A conceptual model should state the simplifying limiting assumptions that translate the real world into a mathematical formulation of the processes that control contaminant movement. The conceptual model for water movement and dissolved mass transport used in this study is illustrated in Figure 1.

\subsubsection{Operational Accidents}

3.3.1.1 Crane Accidents. The severity level of this type of accident depends on the impact or crush force to which the container is subjected. Assuming that the liner weighs $6,000 \mathrm{lb}$, the force of impact would be 27,000 Newtons. This is Severity Level III with a release fraction of 0.1 that, when combined with the aerosol fraction of 0.1 , yields a volume release fraction of 0.01 . A dispersibility factor or respirable fraction of 0.05 for small powder is assumed.

3.3.1.2 Earthquakes. It was assumed that the earthquake causes the crane beam to drop, crushing 14 containers and releasing 0.001 of the radioactivity in the containers (per the North Carolina SAR). It was also assumed that the Waste Packaging Building is destroyed and that the airborne material could reach a member of the general public in the unrestricted area. Atmospheric dispersion was modeled for class $\mathrm{F}$ stability conditions with $2-\mathrm{m} / \mathrm{s}$ wind speed in the direction of the nearest facility boundary, where a member of the public was assumed to remain for the duration of the passage of the contaminated plume.

\subsubsection{Post-Closure Intruders}

3.3.2.1 100 Years Post-Closure. For the 100-year post-closure intrusion scenario, penetrating radiation from buried waste causes doses to the intruders. The doses were estimated assuming 1/3 time occupancy $(2922 \mathrm{~h} / \mathrm{y})$ of the basement of a house that uses the waste module roof as the basement floor. For modeling purposes, the waste volume was treated as a rectangular prism with $2 \mathrm{ft}$ of concrete shielding provided over the entire extent of the upper surface of the waste. Additional shielding that would be provided by the soil surrounding the basement walls was not included in the model.

3.3.2.2 500 Years Post-Closure. Doses to the intruder for the 500-year post-closure scenario would be caused by the undisturbed waste under and surrounding the lower part of the foundation, and by waste relocated to the ground surface by excavation of a basement for a house. Other processes that might cause upward migration of the waste from deep soil are considered to be of minor importance as compared to excavation. The GENII model for estimating doses to an intruder requires input of soil concentrations -- these soil concentrations are derived from the estimated amounts of waste radioactivity brought to the surface by excavation work. A complete description of the GENII model for estimating intruder doses is provided in Napier et al. (1988). 
Doses caused by penetrating radiation from the undisturbed waste were modeled by subdividing the source volume into subvolumes that could be more easily managed with MicroShield. The shielding provided by the concrete foundation was accounted for in the modeling.

A 10 foot $(3.05 \mathrm{~m})$ deep excavation was assumed to be made in the center of a module. This results in the transfer of a $0.3-\mathrm{m}$ by $10-\mathrm{m}$ by $10-\mathrm{m}\left(30 \mathrm{~m}^{3}\right)$ volume of waste to the surface soil. The excavated soil and waste was assumed to be evenly distributed around the house in a $2,200-\mathrm{m}^{2}$ yard, resulting in a contaminated layer of soil approximately 6 in. thick.

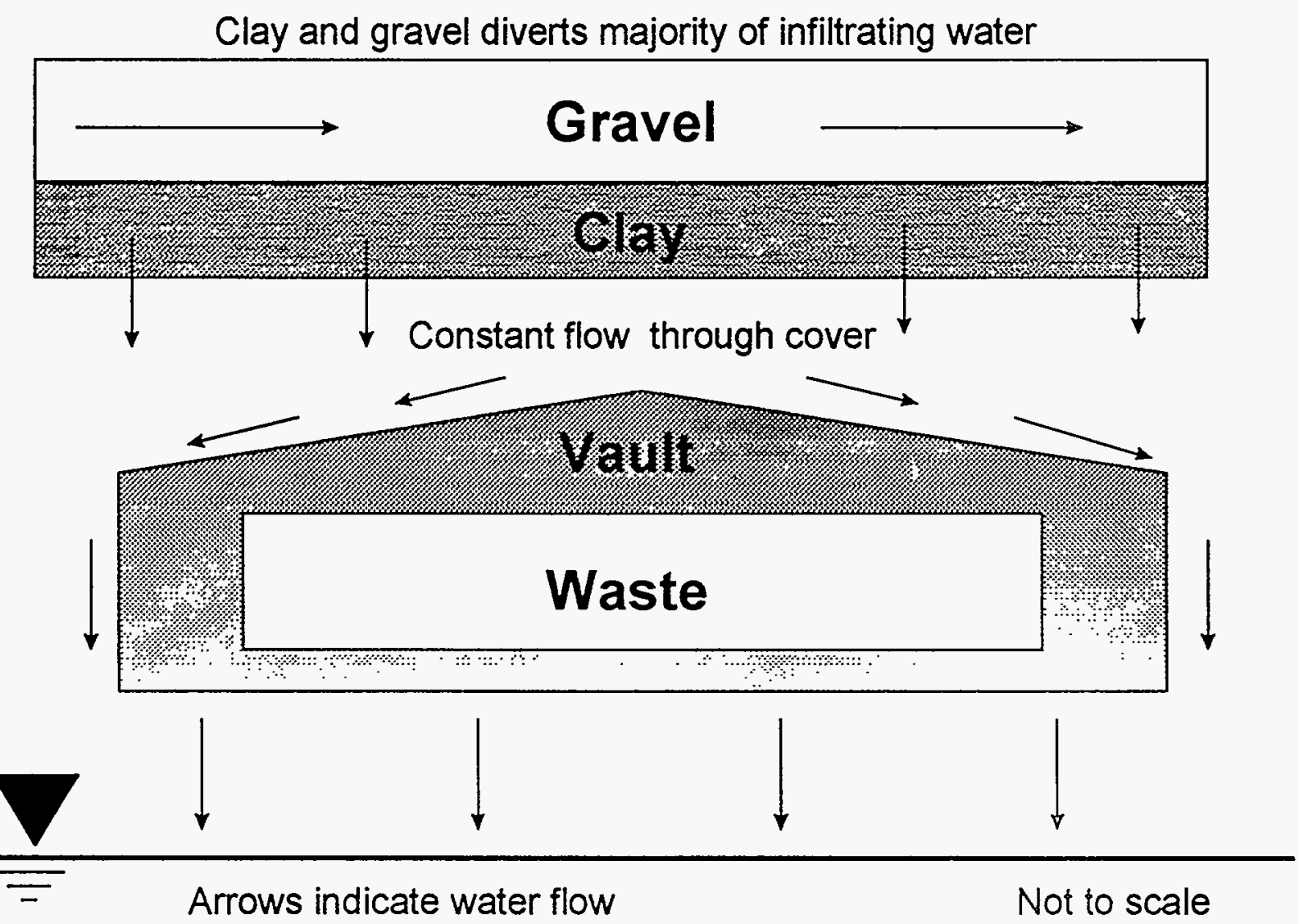

Figure 1. Conceptual model for hypothetical waste facility. The gravel and clay cap divert most of the infiltrating water away from the concrete vault. A small amount of water migrates through the cap and concrete vault and into the waste while the facility is intact. After complete failure (1000 years), water is nut diverted by the cap or restricted by the concrete vault and is allowed to migrate freely through the facility.

\subsubsection{Post-Operational Groundwater}

The conceptual model developed for the groundwater pathway analysis is a simplification of a natural groundwater system, thus providing feasible solutions, yet is complex enough to provide adequate physical description. The groundwater pathway system in this analysis is simplified by dividing the modeling domain into three parts; a waste zone, unsaturated zone, and aquifer. 
The waste zone consists of waste-containing vaults surrounded by a thin layer of sand and buried in homogeneous, isotropic, unconsolidated backfill and native soil (Figure 1). The unsaturated zone consists of the native soil between the base of the backfill soil and the upper surface of the aquifer. The backfill, native soil, and aquifer materials are assumed to have the properties of loam. The model is simplified by examining a single vault. Although a single conceptual model describes the waste and unsaturated zone processes for this analysis, two sets of numerical models were actually required since the designs differ for class $A$ and $B$ waste vaults and class $C$ waste vaults. Land surface provides the physical upper boundary of the system, while the top of the hypothetical aquifer provides the lower boundary. Natural precipitation infiltrates from land surface and reaches the buried waste vault. Contaminants leach from the vault at rates proportional to the amount of water that comes in contact with the waste, and the sorptive properties of the nuclide. The amount of water that comes in contact with waste is dependent on the integrity of the vault

The conceptual model for the waste zone includes three integrity scenarios. The vault is assumed to be fully intact for the first 500 years of service. From 500 years to 1,000 years following closure of the facility, it is assumed that several fractures have formed in the vault, providing increased contact between infiltrating water and waste. After 1,000 years, the vault fails completely and water is allowed to infiltrate as if no barriers or containment existed.

Based on the assumptions of a uniform mixture of vault contents, uniform vault material properties, and homogeneous and isotropic soil properties, it was determined that the waste zone system could adequately be described by a two-dimensional profile model that considers the width and height of a waste vault but not the vault's length. Therefore, all input fluxes and resulting concentrations required correction to a unit length basis; however, this proved less cumbersome and less resource-intensive than modeling the system in all three spatial dimensions. The design of both classes of vaults is symmetrical with respect to vault width. The apex of the sloped roof is at the centerline of the vault (Figure 1). It was assumed that this symmetry of design, when constructed of uniform materials and placed in homogeneous, isotropic medium, would perform with similar symmetry. It was therefore determined that the numerical model setup could be further simplified by limiting the conceptual model to only one-half of a vault. The final conceptual model for the vault and unsaturated zone is depicted in Figure 1.

To complete the groundwater pathway model, the contaminant fluxes from the waste and unsaturated models are used as input to the saturated zone model. The saturated portion conceptually consists of a homogeneous, isotropic fully saturated porous media of finite thickness where flow is uniform and constant throughout the model domain. The system is bounded at top by the aquifer surface. The flux of leached radionuclides provides a transient mass flux into the aquifer system. The lower boundary of the aquifer was arbitrarily chosen as the typical well screen thickness in drinking water wells. The upgradient boundary to this model is defined by the natural flux of groundwater into the system while the downgradient boundary consists of the natural flux of groundwater out of the system. Instantaneous complete vertical mixing in the aquifer is assumed.

Concentrations are evaluated at a point, $100 \mathrm{~m}$ downgradient from the downgradient edge of the waste facility. A receptor is assumed to drill a well at that location. The conceptual model provides for advective transport and dispersion of contaminants from where they enter the aquifer (below the source) to the downgradient location of the receptor well. 


\subsubsection{Screening}

The inventory of LLW radionuclides was subjected to a screening procedure in order to reduce the number of radionuclides for which a detailed analysis was required. Screening was based on an absolute method where groundwater concentrations and the 50-year Committed Effective Dose Equivalent (CEDE) are conservatively estimated and compared to a set criteria. The criteria used in this analysis was $4 \mathrm{mrem} / \mathrm{y}$ CEDE for the beta-gamma emitting nuclides. The $4 \mathrm{mrem} / \mathrm{y}$ standard as stated in 40 CFR 141 is based on a whole body dose computed using the International Commission on Radiation Protection (ICRP) 2 methodology. Proposed changes to the drinking water standard as stated in the July 18, 1991 Federal Register uses the same dose limit ( $4 \mathrm{mrem} / \mathrm{y})$, but computes the dose using ICRP 30 methodology, which calculated the 50-year CEDE. For this analysis, we have used the more recent ICRP 30 methodology to compute the 50-year CEDE and compared this value to the $4 \mathrm{mrem} / \mathrm{y}$ limit. The receptor is assumed to drink 2 Liters of water per day for 365 days per year. Actinides were also compared to this standard, along with the limit for maximum alpha activity in water of 15 $\mathrm{pCi} / \mathrm{L}$ (exluding uranium and radon) and maximum Ra-226/Ra-228 concentrations of $5 \mathrm{pCi} / \mathrm{L}$ as stated in 40 CFR 141.

\subsection{Models and Assumptions}

Five computer models were used for this analysis. For the screening of contaminants via the groundwater pathway, the GWSCREEN (Rood, 1994) code was used. For the detailed groundwater pathway analysis, the PORFLOW code (Runchal and Sagar, 1991) was used to model contaminant leaching from the vaults, transport through the unsaturated zone, and transport in the aquifer. Intruder doses were calculated using the GENII code (Napier et al., 1988) and MicroShield code. Operational accident doses were calculated with the RSAC5 code (Wenzel, 1995). Each code is described at the end of this section. Input parameters and model assumptions are also discussed in this section.

\subsubsection{Soil Hydraulic Parameters}

The soil hydraulic parameters define the interaction between the water and the soil matrix. Thin layers of soils with large grains, such as sand and gravel, provide less resistance to vertical water flow than layers with smaller pore sizes, such as clay, concrete, and grout material. There exists functional relationships describing the interaction between water and the soil such as given by van Genuchten (1978) and presented here in the following equations.

$$
\theta=\theta_{r}-\frac{\left(\theta_{s}-\theta_{r}\right)}{\left[1-(\alpha h)^{n}\right]^{l-\frac{l}{n}}}
$$

where

$$
\begin{array}{rll}
\mathrm{h} & = & \text { suction head } \\
\theta & = & \text { volumetric moisture content } \\
\theta_{r} & = & \text { residual moisture content } \\
\theta_{s} & = & \text { effective porosity } \\
\mathrm{n} & =\text { pore-size distribution index } \\
\alpha & =\text { inverse air-entry potential. }
\end{array}
$$


The equation for the hydraulic conductivity curve is

$$
K(h)=K_{s} \frac{\left(1-(\alpha h)^{n-1}\left[1+(\alpha h)^{n}\right]^{1-1 / n}\right)^{2}}{\left[1+(\alpha h)^{n}\right]^{\alpha(1-l / n)}}
$$

where

$$
\begin{array}{lll}
\mathrm{K}(\mathrm{h}) & = & \text { unsaturated hydraulic conductivity } \\
\mathrm{K}_{\mathrm{s}}= & \text { saturated hydraulic conductivity } \\
\mathrm{l}= & \text { pore interaction term. }
\end{array}
$$

Thus, a fine-grained material will tend to retard the movement of infiltrating water more than a coarsegrained material; this, of course, assumes a homogeneous material. Proper characterization of the porous media to be used in the facility would have to be done before a final analysis could be completed. However, for the purpose of this analysis, the following soil moisture characteristics described in the ensuing sections were assumed to represent the soils at the facility. Table 2 lists each soil type and the source for the parameters that define the soil moisture characteristic curve.

3.4.1.1 Loam. Carsel and Parrish (1988) used a statistical multiple regression technique for estimating hydraulic parameters for various soils types. Because the actual location of the hypothetical site is not yet known, proxy data for a typical sandy clay loam was selected. However, since the soil will be compacted as it is backfilled around the concrete vaults, it is reasonable to expect the hydraulic properties to change during this compaction. The compacted hydraulic parameters were determined in four steps from the uncompacted parameters. First, the porosity and the pore size distribution index (n) were reduced $25 \%$ to account for the reduction of pore space sizes during compaction. After compaction, the reduction in pore sizes will cause the soil to behave similar to a finer textured soil, which will tend to drain through larger range of capillary pressures corresponding to a soil with a smaller $\mathrm{n}$ value.

Table 2. Hydraulic parameter sources for simulation of unsaturated zone performance analysis.

\begin{tabular}{ll}
\multicolumn{1}{c}{ Material Type } & \multicolumn{1}{c}{ Source of Hydraulic Parameters } \\
\hline $\begin{array}{l}\text { Compacted backfill } \\
\text { (loam) }\end{array}$ & $\begin{array}{l}\text { Calculated using technique described by } \\
\text { Carsel and Parrish (1988) } \\
\text { Fractures }\end{array}$ \\
Sand & Assumed \\
Concrete & IAEA (1995) \\
Waste & Yu et al. (1993) \\
\hline
\end{tabular}

Second, the compacted bulk density was calculated from the particle density and compacted porosity using the relation 


$$
\rho_{p}=\frac{\rho_{b c}}{l-\theta_{s}}
$$

where

$$
\begin{aligned}
& \rho_{p}=\text { particle density } \\
& \rho_{b c}=\text { compacted bulk density } \\
& \theta_{s}=\text { compacted porosity. }
\end{aligned}
$$

Third, the inverse air entry potential ( $\alpha$ ) in Equation (3) was calculated from the uncompacted loam air entry potential and from an empirical relation (Campbell, 1985). The relation is

$$
\psi_{e}=\psi_{e s}\left(\rho_{b c} / \rho_{b u c}\right)^{0.67 b}
$$

where

$$
\begin{aligned}
& \psi_{e s}=\text { uncompacted silt air-entry potential } \\
& \rho_{b c}=\text { compacted bulk density } \\
& \rho_{b u c}=\text { uncompacted bulk density } \\
& b=-2 \psi_{e s}+0.2 \sigma_{g} \text { in which } \sigma_{g} \text { is the particle size geometric standard deviation. }
\end{aligned}
$$

Fourth, the saturated hydraulic conductivity was reduced to represent the reduced porosity and increased tortuosity. The residual moisture content was unchanged after compaction because the residual moisture content is a function of the total surface area of the soil particles, which is not changed by compaction. Table 3 lists the hydraulic parameters used in Equations (1) and (2) for each soil type.

Table 3. Hydraulic parameter values for each material type.

\begin{tabular}{lcccccc}
\hline \multicolumn{1}{c}{ Soil Type } & $\begin{array}{c}\mathrm{K}_{\mathrm{s}} \\
(\mathrm{cm} / \mathrm{s})\end{array}$ & $\begin{array}{c}\theta_{s} \\
(\mathrm{cc} / \mathrm{cc})\end{array}$ & $\begin{array}{c}\theta_{r} \\
(\mathrm{cc} / \mathrm{cc})\end{array}$ & $\begin{array}{c}\alpha \\
(1 / \mathrm{cm})\end{array}$ & $\mathrm{n}$ & $\begin{array}{c}\rho_{b} \\
(\mathrm{~g} / \mathrm{cc})\end{array}$ \\
\hline $\begin{array}{l}\text { Compacted } \\
\text { and loam }\end{array}$ & $3.64 \times 10^{-4}$ & 0.39 & 0.1 & 0.059 & 1.48 & 1.6 \\
Fractures & $5.00 \times 10^{-3}$ & 0.38 & 0.372 & $5.98 \times 10^{-4}$ & 3.43 & 1.6 \\
Sand & $5.00 \times 10^{-3}$ & 0.38 & 0.04 & 0.155 & 2.43 & 1.6 \\
Concrete & $1.00 \times 10^{-10}$ & 0.15 & 0.147 & $5.98 \times 10^{-4}$ & 3.43 & 1.6 \\
Waste & $1.00 \times 10^{-10}$ & 0.50 & 0.497 & $5.98 \times 10^{-4}$ & 3.43 & 0.8 \\
\hline
\end{tabular}

3.4.1.2 Fractures. The concrete degradation process developed for this analysis assumes fractures in module concrete walls begin to affect facility performance approximately 500 years after they were built. The introduction of fractures into the simulation model after the first 500 years necessitated defining a moisture characteristic curve that allows water to flow through an element of 
the model domain that had previously been defined as concrete or waste. The flow and transport code PORFLOW, used to prepare these simulations, implements fractures using an equivalent porous media approach. As a result, the fracture and surrounding matrix capillary pressures are the same. The moisture characteristic curve defined for the fracture zone must allow for flow under capillary pressures common to the concrete in the unsaturated zone. Defining the fracture's moisture characteristic curve parameters with the values used for the larger pore-space sand results in a tension head in the fracture that is insufficient to draw flow into the fracture; likewise, if the fracture is described using concrete parameters only, the resulting hydraulic conductivity does not allow sufficient flow through the fracture. To circumvent the code's equivalent porous media implementation of fractures, it was necessary to assign the fracture's hydraulic conductivity a value similar to that of sand and to assign the moisture characteristic curve parameters the values used for concrete. By doing this, the fracture remains saturated under high capillary pressures, yet is able to transmit water at the higher hydraulic conductivity rate. The hydraulic conductivities and moisture characteristic curve parameters used to represent the various materials are summarized in Table 3.

3.4.1.3 Sand. The purpose for the sand placed around the vault was to drain water away from the system. As with the other materials, it was not known the exact type of material that will be used in the final design, so we defined generic parameters to this material.

3.4.1.4 Concrete and Waste. A low-level radioactive waste containment system consisting of a concrete outer shell and concrete overpacks around the waste with sand backfill has been developed for use in disposal in Pennsylvania. The concrete overpacks provide primary containment during vault operations, and the concrete shell provides structural stability for the disposal and will also act as a hydrologic barrier until failure due to fracturing or other degradation processes. Models have been applied to provide estimates of concrete degradation and rebar corrosion due to reactions with soil water chemical constituents in contact with the concrete vault. The models described by Walton et al. (1990) were used to estimate concrete degradation and rebar corrosion processes. Concrete degradation processes considered are (a) magnesium and sulfate attack, (b) calcium leaching (both geologically and concrete controlled), and (c) carbonation. Rebar corrosion due to chloride attack and oxygen-limited corrosion is also considered for the vaults. A brief discussion of each process considered is provided (Appendix $B$ ), along with estimated degradation and corrosion rates for the concrete and rebar, respectively.

The degradation processes have been modeled separately rather than integrated because degradation is typically dominated by a single process. Parametric estimates of degradation are used to evaluate wide ranges of scenarios. Through-running fractures are assumed to occur at 500 years, and complete hydrologic failure occurs at 1,000 years as a first approximation. This assumption was based on calculations made for the Savannah River Plant Intermediate Level Non-Tritium waste vaults. The degradation model estimates support the assumption that the vaults will provide the described hydrologic isolation under the assumed conditions. Magnesium and sulfate attack has the greatest impact on the concrete and is predicted to reach the rebar in approximately 3,000 years. This would result in more rapid corrosion of the rebar, but will not impact the vault performance if the vault interior is backfilled (i.e., limited tensile stress).

Results indicate that moving the steel reinforcement deeper into the concrete would enhance the long-term durability of the vaults by keeping the passivation layer stable for a longer period of time. The concrete mix design might be modified after site selection to optimize the long-term performance of the vault. Because the vault design has not been finalized, the effort for this level of calculation is not warranted. 
The concrete hydraulic parameters were assumed to be similar to those used for the concrete vaults constructed to contain low and intermediate waste at the Savannah River Site (Yu et al., 1993). Because the waste is placed in concrete containers and the containers are grouted into the vaults, it was assumed that the waste's hydrological properties were the same as the concrete. Thus, all of the parameters were the same except for the bulk density. The bulk density of the waste was assigned half the value in the waste as that for the concrete. The rationale for this was to account for voids in the concrete waste containers and the waste itself. The actual values for the hydraulic parameters are also listed in Table 3.

Table 4. Sorption coefficients $(\mathrm{mL} / \mathrm{g})$ for loam, concrete, and grouted waste

\begin{tabular}{|c|c|c|c|}
\hline Element & $\begin{array}{l}\text { Backfill (loam) } \\
\text { and aquifer }^{d}\end{array}$ & Concrete & Waste \\
\hline Actinium & 15 & 0 & 0 \\
\hline Americium & 9,600 & $5,000^{\mathrm{a}}$ & $5,000^{a}$ \\
\hline Antimony & 150 & 0 & 0 \\
\hline Cadmium & 40 & $100^{\mathrm{b}}$ & $100^{\mathrm{b}}$ \\
\hline Carbon & 20 & $100^{\mathrm{a}}$ & $100^{\mathrm{a}}$ \\
\hline Cesium & 4600 & $20^{c}$ & $20^{c}$ \\
\hline Cobalt & 1,300 & $100^{\mathrm{b}}$ & $100^{\mathrm{b}}$ \\
\hline Curium & 18,000 & $100^{\mathrm{b}}$ & $100^{\mathrm{b}}$ \\
\hline Europium & 0 & $5,000^{\mathrm{a}}$ & $5,000^{2}$ \\
\hline Hydrogen (Tritium) & 0 & 0 & 0 \\
\hline Iodine & 5 & $5^{a}$ & $5^{\mathrm{a}}$ \\
\hline Iron & 800 & $100^{\mathrm{d}}$ & $100^{d}$ \\
\hline Lead & 0 & 16,000 & 16,000 \\
\hline Neptunium & 25 & $5,000^{\mathrm{a}}$ & $5,000^{\mathrm{a}}$ \\
\hline Niobium & 550 & $1,000^{\mathrm{a}}$ & $1,000^{\mathrm{a}}$ \\
\hline Nickel & 300 & $100^{a}$ & $100^{a}$ \\
\hline Plutonium & 1200 & $5,000^{\mathrm{a}}$ & $5,000^{\mathrm{a}}$ \\
\hline Promethium & 0 & $5,000^{a}$ & $5,000^{\mathrm{a}}$ \\
\hline Protactinium & 15 & $1,500^{\mathrm{a}}$ & $1,500^{\mathrm{a}}$ \\
\hline Radium & 36,000 & $100^{a}$ & $100^{\mathrm{a}}$ \\
\hline Sodium & 0 & 0 & 0 \\
\hline Strontium & 20 & $3^{a}$ & $3^{\mathrm{a}}$ \\
\hline Technetium & 0.1 & $1.0^{\mathrm{c}}$ & $1.0^{\mathrm{c}}$ \\
\hline Thorium & 3,300 & $5,000^{\mathrm{a}}$ & $5000^{\mathrm{a}}$ \\
\hline Uranium & 15 & $1,500^{\mathrm{a}}$ & $1,500^{\mathrm{a}}$ \\
\hline \multicolumn{4}{|l|}{ Krupka 1996} \\
\hline b DOE/ID-10521/2 1996 & & & \\
\hline c Allard 1985 & & & \\
\hline d Sheppard and Thibault 1990 & & & \\
\hline
\end{tabular}




\subsubsection{Sorption Coefficients}

Transport of radionuclides out of the vaults and in the subsurface environment is modeled using distribution coefficients to describe the partitioning between the aqueous and solid phases in the porous media. Contaminants that are released from their primary containment will come into contact with the concrete vault. The current mix design consists of Type II OPC with sand, fly ash, and aggregate. The properties of concrete provide a good transport barrier for many radionuclides (Table 4) and many of the long-lived radionuclides are readily immobilized (highly sorbed) within the concrete. Addition of slag into the cement would improve the retention of reducible contaminants like technetium. This may be an option to enhance performance if technetium is shown to be a problem.

The concrete $\mathrm{K}_{d}$ 's respond to changes in the concrete $\mathrm{pH}$. However, the change in concrete $\mathrm{pH}$ is gradual (thousands to millions of years [Walton et al., 1990]), and variation in the chemical control on contaminants will remain stable for an extended period of time. The location of the facility has not been selected, and site-specific distribution coefficients for the backfill and porous media are not available. Because of the lack of site-specific information, conservative $K_{d}$ values from available literature were selected for the backfill and porous media (Table 4). For further information on $\mathrm{K}_{d}$ selection for Performance Assessments, see Appendix C.

\subsubsection{Initial and Boundary Conditions}

For the flow and transport simulation in the unsaturated zone model, the saturation level of the backfill and native soil surrounding the waste vault was set to a value of 1.0 ; that is, the surrounding backfill and soil were assumed to be fully saturated and, as the simulations progressed, the soil was allowed to come to a value of saturation consistent with the infiltration rate of precipitation and the specified moisture characteristic curve defined for this material type. Initial contaminant conditions were simulated with unit concentration to minimize the number of simulations. The vertical boundaries of the unsaturated zone were fixed spatially and temporally as no-flux boundaries. The top horizontal boundary was set as a fixed flux of $58 \mathrm{~cm} / \mathrm{yr}$ of infiltrating precipitation. The bottom horizontal boundary was simulated as specified-concentration boundary; that is, the contaminant concentrations just beneath this boundary were always set to zero. This, in effect, conservatively maximizes the flux of contaminants to the aquifer.

Initial conditions for contaminant transport in the saturated zone were based largely upon output from the unsaturated zone simulations. The upper boundary of the aquifer was established as the output flux of the unsaturated simulations. The lower boundary was specified as no-flux. The vertical upgradient and downgradient boundaries were each established as specified-concentration boundaries, with the contaminant concentrations just outside the boundaries specified as zero concentration. This conservatively maximized transport of contaminants to the downgradient receptor locations. Groundwater velocity in the horizontal direction was specified as $25 \mathrm{~m} / \mathrm{yr}$ throughout the aquifer, while the vertical velocity was set to $0 \mathrm{~m} / \mathrm{y} r$. Initial conditions for contaminant concentrations were always set as zero-concentration everywhere in the aquifer. The flow field was assumed to be uniform throughout the aquifer.

3.4.3.1 Screening Model Assumptions The groundwater contaminant transport code, GWSCREEN (Rood 1994) was used to perform the screening calculations. The following simplifying, and conservative assumptions were made for these calculations:

- All waste was assumed to be placed instantaneously into a volume comprising the open pit area of the waste facility. 
- The waste was assumed to be homogeneously mixed with a soil matrix that has the same hydrologic properties as backfill soil (loam) and the same sorptive properties of the waste grout (cement).

- No credit was taken for engineered barriers to restrict water movement in and out of the waste.

- Leaching of radionuclides from the soil was described by first-order kinetics which is a function of the net infiltration and sorptive properties of the nuclide.

- The waste vaults were placed directly over the aquifer.

- Transport in the saturated zone is described by a semi-analytical solution to the advectiondispersion equation for an aquifer of infinite lateral extent, finite thickness, and constant, steadystate flow.

- A receptor was placed at the edge of the waste facility buffer zone which is 100 downgradient from the downgradient edge of the waste. This distance was $405 \mathrm{~m}$ downgradient from the center of the $A B$ vaults and $125 \mathrm{~m}$ downgradient from the center of the $C$ vaults.

- The receptor was assumed to drink $2 \mathrm{~L}$ of water per day for 365 days per year.

- Impacts are calculated for the year the groundwater concentration peaks

- Radioactive progeny are assumed to travel at the same rate of the pare.1t.

Values for GWSCREEN input variables are summarized in Table 5. GWSCREEN output files for these screening calculations are presented in Appendix D. A prescreening procedure was applied to the inventory prior to running GWSCREEN. Any nuclide with a half-life less than 1 year was eliminated from consideration. Nuclides with half-lives less than 1 year are essentially decayed away at the end of the 100 year institutional control period.

Table 5. Input parameters for the GWSCREEN screening simulations.

\begin{tabular}{lcc}
\hline Parameter Name & Value for AB Vaults & Value for C Vaults \\
\hline Source length parallel to groundwater flow $(\mathrm{m})$ & 610 & 49 \\
Source width perpendicular to groundwater flow $(\mathrm{m})$ & 122 & 24 \\
Source thickness $(\mathrm{m})$ & 7.6 & 3 \\
Bulk Density of source material $\left(\mathrm{g} / \mathrm{cm}^{3}\right)$ & 1.6 & 1.6 \\
Percolation (infiltration) rate $(\mathrm{m} / \mathrm{y})$ & 0.58 & 0.58 \\
$\alpha$ for source material $(1 / \mathrm{cm})$ & 0.059 & 0.059 \\
n for source material & 1.48 & 1.48 \\
Saturated hydraulic conductivity for source material $(\mathrm{m} / \mathrm{y})$ & 114.8 & 114.8 \\
Residual moisture content for source material & 0.1 & 0.1 \\
Total porosity for source material $\left(\mathrm{m}^{3} / \mathrm{m}^{3}\right)$ & 0.39 & 0.39 \\
Aquifer pore velocity $(\mathrm{m} / \mathrm{y})$ & 62.5 & 62.5 \\
Longitudinal dispersivity $(\mathrm{m})$ & 10 & 10 \\
Transverse dispersivity $(\mathrm{m})$ & 5 & 5 \\
Well screen thickness $(\mathrm{m})$ & 15 & 15 \\
Aquifer bulk density $\left(\mathrm{g} / \mathrm{cm}^{3}\right)$ & 1.6 & 1.6 \\
\hline
\end{tabular}




\subsubsection{Numerical Models}

3.4.4.1 GWSCREEN The GWSCREEN model is a groundwater assessment code that was developed for assessment of the groundwater pathway from the leaching of radioactive and nonradioactive contaminants from surface or buried sources. The code was designed for implementation in the Track 1 and Track 2 assessment of Comprehensive Environmental Response, Compensation, and Liability Act (CERCLA) sites identified as low probability hazard at the Idaho National Engineering Laboratory. The code has been adapted to many other applications including low-level waste performance assessment.

The model calculates the limiting soil concentration and inventory so that after leaching and transport of the contaminant to the aquifer, regulatory contaminant levels in groundwater are not exceeded. It can also calculate groundwater concentrations and corresponding health impacts (radiological dose) as a function of time and distance away from the source. The code uses a mass conservation approach to model three processes: (a) contaminant release from a source volume, (b) contaminant transport in the unsaturated zone, and (c) contaminant transport in the saturated zone. The source model considers the sorptive properties and solubility of the contaminant. Transport in the unsaturated zone is described by a plug flow model. Transport in the saturated zone is calculated using a semi-analytical solution to the advection dispersion equation in groundwater for transient mass fluxes. Transport of radioactive progeny is accomplished by assuming the radioactive progeny travel at the same rate as their parent.

GWSCREEN results are comparable with results from other codes using similar algorithms and have also been shown to provide bounding estimates of groundwater concentrations when compared to results from numerical codes. A formal verification and validation report was completed (Smith and Whitaker 1993). GWSCREEN was not intended to be a predictive tool. Rather, it provides a framework to calculate bounding impacts to groundwater from the leaching of surficial and buried contamination.

3.4.4.2 PORFLOW. The PORFLOW computer code, Version 2.39 (Runchal and Sagar 1991) was developed to evaluate hazardous and radioactive waste problens. The computer code is based on the numerical solution of the general equations for (a) time-dependent, porous flow, (b) transport of a reactive solute, and (c) coupled heat transport. The governing equations describing these processes are approximated by an integrated finite-difference technique. Both direct and iterative solution approaches are available and can be used to solve the finite-difference equations.

The code is capable of solving for fluid flow, heat, and mass transport in porous media, which can be heterogenous, anisotropic, variably saturated, and/or discreetly fractured. Specific capabilities of the code include the ability to simulate the following: multiple phases, up to four species at a time, chemical reactions, four-member chain decay with differential transport of decay members, arbitrary sources and sinks, and variable density as a function of either concentration or temperature.

Limitations of the model include (a) single temperature for all phases, (b) one-phase heat and mass transport so that there is no heat transfer between phases, (c) reversible linear Freundlich isotherm to describe partitioning, and (d) one-way chain decay so that daughter products can only occur along one chain per simulation.

An earlier version of the PORFLOW code, called PORFLO-3, was verified against a number of known analytical solutions and benchmarked tested by making code-to-code comparisons for several 
flow and transport simulations. The results of the verification and benchmark testing are documented in Magnuson et al. (1990).

3.4.4.3 MicroShield. The MicroShield model (Grove Engineering, 1992) was used to estimate doses to individuals occupying the basement of a house built such that the basement floor slab is the existing roof of a class A and B waste module. The waste in the module was the only source of penetrating radiation accounted for in the MicroShield calculations (penetrating radiation from surface soil contamination is accounted for by GENII, see Section 3.4.3.4). The inventory in the module was estimated by taking the entire class A and B inventory, calculating the inventory following 100 years of decay, and dividing the decayed inventory by the projected waste volume. The changes in the inventories of the waste radionuclides caused by decay and daughter ingrowth were evaluated using the decay routine in MicroShield. Because of the geometry of the source and basement, the penetrating radiation doses were evaluated for several sub-volumes and summed to arrive at the total dose. The same approach was used to estimate penetrating radiation doses for the intrusion scenario at 500 years.

MicroShield was also used to estimate doses for the crane accident scenarios (occurring during operations). In these scenarios, a waste container is either suspended from the crane with a small shield, or dropped and left temporarily unshielded, but not breached. The waste container was assumed to be a $97-\mathrm{ft}^{3}$ resin liner with the radionuclide composition specified in the North Carolina SAR, generating a $500-\mathrm{r} / \mathrm{h}$ field at $10 \mathrm{~cm}$ from the surface (midline of axis). The inventory of the container was determined by scaling the total activity in the proportions specified in the North Carolina SAR to generate the $500-\mathrm{r} / \mathrm{h}$ field specified in the North Carolina SAR.

3.4.4.4 GENII. The GENII model (Napier et al., 1988) accounts for internal and penetrating radiation caused by contaminated surface soil. The concentration of radionuclides in waste excavated 500 years post-closure was estimated using RSAC-5. Electronic data transfers were used to copy the RSAC-5 inventory output to the GENII input.

Penetrating radiation doses were estimated using a point-kernel subroutine in GENII that is based on the IsoShield model, which is similar to MicroShield. GENII also calculates doses caused by intake of radionuclides in plant and animal products that are consumed by individuals, inhalation of resuspended soil, and inadvertent ingestion of soil. Intakes of radionuclides in foodstuffs is modeled using U.S. NRC Regulatory Guide 1.07 methods and a set of environmental parameters developed for the Hanford site in western Washington state.

3.4.4.5 RSAC-5. The RSAC-5 code calculates doses caused by brief releases of radionuclides to the atmosphere. RSAC-5 has been validated and verified in accordance with ANSI/ANS-10.4 (1987), "American National Standard Guidelines for the Verification and Validation of Scientific and Engineering Programs for the Nuclear Industry." The code performs Gaussian plume dispersion modeling applying Pasquill-Gifford diffusion parameters, and calculates doses through inhalation, ingestion, immersion, ground surface, and cloud gamma radiation. The RSAC- 5 code was used to calculate the doses caused by the fire and earthquake accident scenarios. RSAC- 5 was also used to generate the decayed waste inventories for input to GENII.

\section{RESULTS OF ANALYSIS}

\subsection{Operational Accidents}

Table 6 summarizes the results of analysis of accidents at the Pennsylvania disposal facility. The 
results are presented for intake through inhalation, exposure to penetrating radiation caused by immersion in contaminated air, and exposure to penetrating radiation emitted by waste that is contained but is either closer than usual to the dose receptor, or unshielded, i.e., direct radiation.

\subsection{Post-Closure Intrusion}

Table 7 summarizes the results of analysis of intrusion into the Pennsylvania disposal facility following closure. The results are presented as calculated by GENII for intake through inhalation, exposure to penetrating radiation caused by immersion in contaminated air, and exposure to direct

Table 6. Off-site doses (mrem) caused by bounding hypothetical operational accidents at the Pennsylvania disposal facility.

\begin{tabular}{lcccc}
\hline \multicolumn{1}{c}{ Scenario } & $\begin{array}{c}\text { Inhalation } \\
(\mathrm{mrem})\end{array}$ & $\begin{array}{c}\text { Immersion } \\
(\mathrm{mrem})\end{array}$ & $\begin{array}{c}\text { Direct radiation } \\
(\mathrm{mrem})\end{array}$ & $\begin{array}{c}\text { Total } \\
(\mathrm{mrem})\end{array}$ \\
\hline $\begin{array}{l}\text { Fire in waste packaging } \\
\text { building }\end{array}$ & $8.6 \times 10^{-2}$ & $8.2 \times 10^{-4}$ & -- & $8.7 \times 10^{-4}$ \\
$\begin{array}{l}\text { Crane malfunction } \\
\text { suspended container }\end{array}$ & & $1.2 \times 10^{-3}$ & $1.2 \times 10^{-3}$ \\
$\begin{array}{l}\text { Crane malfunction - } \\
\text { dropped container }\end{array}$ & & $2.8 \times 10^{-2}$ & $2.8 \times 10^{-2}$ \\
$\begin{array}{l}\text { Tornado } \\
\text { Earthquake }\end{array}$ & $2.5 \times 10^{-1}$ & $8.3 \times 10^{-3}$ & $4.3 \times 10^{0}$ & $4.3 \times 10^{0}$ \\
\hline
\end{tabular}

Table 7. Doses (mrem) to individuals intruding into the Pennsylvania disposal facility.

\begin{tabular}{|c|c|c|c|c|c|}
\hline & $\begin{array}{l}\text { Ingestion } \\
\text { (mrem) }\end{array}$ & $\begin{array}{l}\text { Inhalation } \\
\text { (mrem) }\end{array}$ & $\begin{array}{l}\text { Direct radiation } \\
\text { surface soil } \\
\quad(\mathrm{mrem})\end{array}$ & $\begin{array}{l}\text { Direct radiation } \\
\text { buried waste } \\
\text { (mrem) }\end{array}$ & $\begin{array}{l}\text { Total EDE } \\
\text { (mrem) }\end{array}$ \\
\hline $\begin{array}{l}\text { Class A and B } \\
100 \text {-year } \\
\text { closure }\end{array}$ & -- & -- & -- & 0.21 & 0.21 \\
\hline $\begin{array}{l}\text { Class C 100-year } \\
\text { post-closure }\end{array}$ & -- & -- & -- & 1.6 & 1.6 \\
\hline $\begin{array}{l}\text { Class A and B } \\
\begin{array}{l}500 \text {-year } \\
\text { closure }\end{array}\end{array}$ & 8.8 & 0.4 & 2.8 & 7.8 & 20 \\
\hline $\begin{array}{l}\text { Class C 500-year } \\
\text { post-closure }\end{array}$ & 337 & 1.8 & 183 & 175 & 697 \\
\hline
\end{tabular}


effective dose equivalent (EDE) for inhalation and ingestion, and the external EDE for immersion and direct radiation. The total EDE is defined as the sum of the committed and external EDEs. The total EDE reported by GENII and presented in Table 6 may not be the exact sum of the committed and external EDEs because of rounding.

Tables 5 and 6 present doses for the 100 year post closure and 500 year post closure scenarios. For the 100 year post closure scenario, $100 \%$ of the dose is from direct radiation. Class $A B$ waste would cause a dose of $0.21 \mathrm{mrem}$ and Class $C$ waste would cause $1.6 \mathrm{mrem}$. For both $A B$ and $C$ waste, most of the dose would be caused by ${ }^{137} \mathrm{Cs}$. For the 500 year post closure scenario, the ingestion pathway was the highest contributor for both $A B$ and $C$ waste. The percent of dose attributed to each pathway is given in Table 8 . The primary ingestion dose contributor was ${ }^{228} \mathrm{Ra}$ for the class $A B$ waste and ${ }^{63} \mathrm{Ni}$ for class $\mathrm{C}$ waste. ${ }^{94} \mathrm{Nb}$ was the major contributor to the direct radiation pathway for class $\mathrm{C}$ waste intrusion at 500 years $-{ }^{228} \mathrm{Ra}$ and progeny were the major direct radiation contributors for class $\mathrm{AB}$ waste.

Table 8. Percentage of dose from each intruder pathway for Class $\mathrm{AB}$ and $\mathrm{C}$ waste.

\begin{tabular}{|l|l|l|l|l|}
\hline Waste Type & Ingestion & Inhalation & $\begin{array}{l}\text { Direct radiation } \\
\text { surface soil }\end{array}$ & $\begin{array}{l}\text { Direct radiation } \\
\text { buried waste }\end{array}$ \\
\hline Class $\mathrm{AB}$ & $61 \%$ & $4 \%$ & $2 \%$ & $33 \%$ \\
\hline Class C & $80 \%$ & $2 \%$ & $7 \%$ & $11 \%$ \\
\hline
\end{tabular}

\subsection{Post-Closure Groundwater Pathway}

Groundwater pathway impacts were evaluated using the assumptions and conceptual model described in Section 3.4.3.1 and the GWSCREEN code. Only nuclides that had a half-life of greater than one year were analyzed. The CEDE for each nuclide in Class AB waste (Table 9) and Class $C$ waste (Table 10) was below the $4 \mathrm{mrem} / \mathrm{y}$ beta gamma dose criteria except ${ }^{3} \mathrm{H}$. The ${ }^{14} \mathrm{C}$ CEDE was close enough to the $4 \mathrm{mrem} / \mathrm{y}$ limit $(3.7 \mathrm{mrem} / \mathrm{y})$ for Class $\mathrm{C}$ waste that this nuclide was included in the more detailed analysis. Tritium had maximum CEDE values of $1.4 \times 10^{4}$ mrem for Class $A B$ waste and $1.3 \times 10^{3} \mathrm{mrem} / \mathrm{y}$ for class $C$ waste. Carbon- 14 had maximum doses of $1.7 \mathrm{mrem} / \mathrm{y}$ and 3.7 $\mathrm{mrem} / \mathrm{y}$ for Class $\mathrm{AB}$ and $\mathrm{C}$ waste respectively. Gross alpha activities were below the $15 \mathrm{pCi} / \mathrm{L}$ limit for all alpha-emitting nuclides (excluding radium and radon) in Class $\mathrm{AB}$ and $\mathrm{C}$ waste (Table 11 and 12) and $\mathrm{Ra}-2267 / 228$ concentrations were below the $5 \mathrm{pCi} / \mathrm{L}$ limit.

Table 9. Maximum committed effective dose equivalents calculated with GWSCREEN for Class AB waste.

\begin{tabular}{|c|c|c|c|c|c|c|c|c|}
\hline Nuclide & Progeny & Inventory & $\begin{array}{c}\text { Factor } \\
\text { (rem/Ci) }\end{array}$ & Half Life & Kd Source & $\begin{array}{c}\mathrm{Kd} \\
\text { Aquifer }\end{array}$ & $\begin{array}{c}\text { CEDE } \\
(\mathrm{mrem} / \mathrm{y})\end{array}$ & Peak Time \\
\hline Am-24l & & $1.29 \mathrm{E}-01$ & $4.50 E+06$ & $4.32 E+02$ & $5.00 \mathrm{E}+03$ & $500 E+03$ & $3.24 \mathrm{E}-13$ & $7.71 \mathrm{E}+03$ \\
\hline & $\mathrm{Np}-237$ & & $3.90 \mathrm{E}+06$ & $2.10 E+06$ & & $2.50 \mathrm{E}+01$ & $2.67 E-09$ & \\
\hline & U-233 & & $2.70 E+05$ & $1.59 E+05$ & & $1.50 \mathrm{E}+01$ & $9.32 E-12$ & \\
\hline & Th-229 & & $3.91 E+06$ & $7.34 \mathrm{E}+03$ & & $3.30 \mathrm{E}+03$ & $1.71 \mathrm{E}-13$ & \\
\hline To & & & & & & & 2.68E-09 & \\
\hline Am-243 & & $1.00 \mathrm{E}-05$ & $4.50 \mathrm{E}+06$ & $7.38 E+03$ & $5.00 \mathrm{E}+03$ & $5.00 \mathrm{E}+03$ & 7.07E-09 & $3.44 \mathrm{E}+04$ \\
\hline
\end{tabular}




\begin{tabular}{|c|c|c|c|c|c|c|c|c|}
\hline Nuclide & Progeny & Inventory & $\begin{array}{c}\text { Factor } \\
\text { (rem/Ci) }\end{array}$ & Half Life & Kd Source & $\begin{array}{c}\mathrm{Kd} \\
\text { Aquifer }\end{array}$ & $\begin{array}{c}\mathrm{CEDE} \\
(\mathrm{mrem} / \mathrm{y})\end{array}$ & Peak Time \\
\hline & $\mathrm{Pu}-239$ & & $4.30 \mathrm{E}+06$ & $2.41 \mathrm{E}+04$ & & $1.20 \mathrm{E}+03$ & $1.05 \mathrm{E}-07$ & \\
\hline & U-235 & & $2.51 E+05$ & $7.04 \mathrm{E}+08$ & & $1.50 \mathrm{E}+01$ & $1.66 \mathrm{E}-11$ & \\
\hline & $\mathrm{Pa}-231$ & & $1.10 E+07$ & $3.28 \mathrm{E}+04$ & & $1.50 \mathrm{E}+01$ & $1.94 \mathrm{E}-10$ & \\
\hline & Ac- 227 & & $1.46 \mathrm{E}+07$ & $2.18 \mathrm{E}+01$ & & $1.50 \mathrm{E}+01$ & $2.56 \mathrm{E}-10$ & \\
\hline \multicolumn{2}{|c|}{ Total } & & & & & & 1.12E-07 & \\
\hline $\mathrm{Ba}-133$ & & $1.85 \mathrm{E}-02$ & $3.20 \mathrm{E}+03$ & $1.05 \mathrm{E}+01$ & $1.00 \mathrm{E}+01$ & $0.00 \mathrm{E}+00$ & $1.32 \mathrm{E}-03$ & $1.03 E+01$ \\
\hline$C-14$ & & $2.31 \mathrm{E}+02$ & $2.10 \mathrm{E}+03$ & $5.70 \mathrm{E}+03$ & $1.00 \mathrm{E}+02$ & $2.00 \mathrm{E}+01$ & $1.70 \mathrm{E}+00$ & $9.35 E+02$ \\
\hline Cd-109 & & $4.20 \mathrm{E}-02$ & $1.20 \mathrm{E}+04$ & $1.27 \mathrm{E}+00$ & $1.00 \mathrm{E}+02$ & $4.00 \mathrm{E}+01$ & $1.61 \mathrm{E}-21$ & $3.64 \mathrm{E}+01$ \\
\hline $\mathrm{Cl}-36$ & & $1.39 \mathrm{E}-01$ & $3.00 \mathrm{E}+03$ & $3.01 \mathrm{E}+05$ & $1.00 \mathrm{E}+00$ & $0.00 \mathrm{E}+00$ & $1.38 \mathrm{E}-01$ & $1.19 E+01$ \\
\hline $\mathrm{Cm}-243$ & & $3.30 \mathrm{E}-02$ & $1.10 \mathrm{E}+05$ & $2.85 E+01$ & $1.00 E+02$ & $1.80 E+04$ & $0.00 \mathrm{E}+00$ & $0.00 \mathrm{E}+00$ \\
\hline & $\mathrm{U}-235$ & & $2.50 \mathrm{E}+05$ & $7.04 \mathrm{E}+11$ & & $1.50 \mathrm{E}+01$ & $0.00 \mathrm{E}+00$ & \\
\hline & $\mathrm{Pa}-2$ & & $1.10 \mathrm{E}+07$ & $3.28 \mathrm{E}+04$ & & $1.50 E+01$ & $0.00 \mathrm{E}+00$ & \\
\hline & Ac- 22 & & $1.46 \mathrm{E}+07$ & $2.18 \mathrm{E}+01$ & & $1.50 \mathrm{E}+01$ & $0.00 \mathrm{E}+00$ & \\
\hline \multicolumn{2}{|c|}{ Total } & & & & & & $0.00 \mathrm{E}+00$ & \\
\hline \multirow[t]{6}{*}{$\mathrm{Cm}-244$} & & $1.32 \mathrm{E}-01$ & $2.30 \mathrm{E}+06$ & $1.81 E+01$ & $1.00 \mathrm{E}+02$ & $1.80 E+04$ & $0.00 \mathrm{E}+00$ & $0.00 \mathrm{E}+00$ \\
\hline & $P u-240$ & & $2.29 \mathrm{E}+06$ & $6.56 \mathrm{E}+03$ & & $1.20 \mathrm{E}+03$ & $0.00 \mathrm{E}+00$ & \\
\hline & U-236 & & $2.50 \mathrm{E}+05$ & $2.43 \mathrm{E}+07$ & & $1.50 \mathrm{E}+01$ & $0.00 \mathrm{E}+00$ & \\
\hline & Th-232 & & $2.80 \mathrm{E}+06$ & $1.41 E+10$ & & $3.30 \mathrm{E}+03$ & $0.00 \mathrm{E}+00$ & \\
\hline & $\mathrm{Ra}-228$ & & $1.20 \mathrm{E}+06$ & $5.75 E+00$ & & $3.60 E+04$ & $0.00 \mathrm{E}+00$ & \\
\hline & Th-228 & & $7.50 \mathrm{E}+05$ & $1.91 E+00$ & & $3.30 E+03$ & $0.00 \mathrm{E}+00$ & \\
\hline \multicolumn{2}{|c|}{ Total } & & & & & & $0.00 \mathrm{E}+00$ & \\
\hline Co-60 & & $4.73 E+04$ & $2.60 E+04$ & $5.75 E+00$ & $1.00 \mathrm{E}+02$ & $1.30 E+03$ & $0.00 \mathrm{E}+00$ & $0.00 \mathrm{E}+00$ \\
\hline Cs- 134 & & $7.63 E+03$ & $7.40 \mathrm{E}+04$ & $2.06 \mathrm{E}+00$ & $2.00 \mathrm{E}+0 \mathrm{I}$ & $4.60 \mathrm{E}+03$ & $0.00 \mathrm{E}+00$ & $0.00 \mathrm{E}+00$ \\
\hline Cs-137 & & $1.27 \mathrm{E}+04$ & $5.00 \mathrm{E}+04$ & $3.00 \mathrm{E}+01$ & $2.00 \mathrm{E}+01$ & $4.60 \mathrm{E}+03$ & $0.00 \mathrm{E}+00$ & $0.00 \mathrm{E}+00$ \\
\hline Eu-155 & & $1.60 \mathrm{E}-03$ & $1.30 \mathrm{E}+03$ & $4.96 \mathrm{E}+00$ & $5.00 \mathrm{E}+03$ & $0.00 \mathrm{E}+00$ & 4.96E-08 & $7.61 \mathrm{E}+00$ \\
\hline Fe-55 & & $1.71 E+05$ & $5.80 \mathrm{E}+01$ & $2.73 E+00$ & $1.00 \mathrm{E}+02$ & $8.00 \mathrm{E}+02$ & $0.00 \mathrm{E}+00$ & $0.00 \mathrm{E}+00$ \\
\hline $\mathrm{H}-3$ & & $3.53 E+05$ & 6.30 & $1.23 \mathrm{E}+01$ & $0.00 \mathrm{E}+00$ & $0.00 \mathrm{E}+00$ & $1.37 \mathrm{E}+04$ & $6.60 \mathrm{E}+00$ \\
\hline $\mathrm{I}-129$ & & $4.11 \mathrm{E}-03$ & $2.80 \mathrm{E}+05$ & $1.57 \mathrm{E}+07$ & $5.00 \mathrm{E}+00$ & $5.00 \mathrm{E}+00$ & $4.58 \mathrm{E}-02$ & $1.95 \mathrm{E}+02$ \\
\hline $\mathrm{Na}-22$ & & $1.36 \mathrm{E}-01$ & $1.20 \mathrm{E}+04$ & $2.60 \mathrm{E}+00$ & $0.00 \mathrm{E}+00$ & $0.00 \mathrm{E}+00$ & $3.49 \mathrm{E}-01$ & $4.06 \mathrm{E}+00$ \\
\hline $\mathrm{Nb}-94$ & & $2.67 \mathrm{E}-01$ & $5.10 E+03$ & $2.00 E+04$ & $1.00 \mathrm{E}+03$ & $5.50 \mathrm{E}+02$ & $1.92 \mathrm{E}-04$ & $1.76 \mathrm{E}+04$ \\
\hline $\mathrm{Ni}-63$ & & $5.03 E+01$ & $5.40 E+01$ & $1.00 \mathrm{E}+02$ & $1.00 \mathrm{E}+02$ & $3.00 \mathrm{E}+02$ & $1.77 \mathrm{E}-08$ & $9.47 \mathrm{E}+02$ \\
\hline $\mathrm{Ni}-59$ & & $5.03 E+01$ & $2.10 E+02$ & $7.50 E+04$ & $1.00 \mathrm{E}+02$ & $3.00 E+02$ & $9.05 \mathrm{E}-03$ & $7.09 \mathrm{E}+03$ \\
\hline $\mathrm{Pb}-210$ & & $7.39 \mathrm{E}-01$ & $6.70 E+06$ & $2.23 \mathrm{E}+01$ & $0.00 \mathrm{E}+00$ & $1.60 \mathrm{E}+04$ & $0.00 \mathrm{E}+00$ & $0.00 \mathrm{E}+00$ \\
\hline Pm-147 & & $9.05 E+00$ & $9.50 E+01$ & $2.62 \mathrm{E}+00$ & $5.00 \mathrm{E}+03$ & $0.00 \mathrm{E}+00$ & $9.59 \mathrm{E}-06$ & $5.05 \mathrm{E}+00$ \\
\hline \multirow[t]{5}{*}{$\mathrm{Pu}-238$} & & $3.15 \mathrm{E}-01$ & $3.80 \mathrm{E}+06$ & $8.77 \mathrm{E}+01$ & $5.00 \mathrm{E}+03$ & $1.20 \mathrm{E}+03$ & $7.77 \mathrm{E}-14$ & $1.69 E+03$ \\
\hline & U-234 & & $2.60 \mathrm{E}+05$ & $2.45 \mathrm{E}+05$ & & $1.50 \mathrm{E}+01$ & $9.58 \mathrm{E}-11$ & \\
\hline & TH-230 & & $5.30 \mathrm{E}+05$ & $7.54 \mathrm{E}+03$ & & $3.30 \mathrm{E}+03$ & $1.21 \mathrm{E}-13$ & \\
\hline & $\mathrm{RA}-226$ & & $1.10 \mathrm{E}+06$ & $1.60 E+03$ & & $3.60 \mathrm{E}+04$ & $6.48 \mathrm{E}-15$ & \\
\hline & PB-210 & & $6.70 E+06$ & $2.23 E+01$ & & $1.60 \mathrm{E}+04$ & $8.56 \mathrm{E}-14$ & \\
\hline Total & & & & & & & $9.61 \mathrm{E}-11$ & \\
\hline \multirow[t]{3}{*}{$\mathrm{Pu}-239$} & & $2.28 \mathrm{E}-01$ & $4.30 \mathrm{E}+06$ & $2.41 E+04$ & $5.00 \mathrm{E}+03$ & $1.20 \mathrm{E}+03$ & $2.07 \mathrm{E}-02$ & $3.32 \mathrm{E}+0$ \\
\hline & U-235 & & $2.50 \mathrm{E}+05$ & $7.04 E+11$ & & $1.50 \mathrm{E}+01$ & 5.17E-09 & \\
\hline & $\mathrm{Pa}-23 \mathrm{I}$ & & $1.10 \mathrm{E}+07$ & $3.28 \mathrm{E}+04$ & & $1.50 \mathrm{E}+01$ & $7.28 \mathrm{E}-08$ & \\
\hline
\end{tabular}




\begin{tabular}{|c|c|c|c|c|c|c|c|c|}
\hline Nuclide & Progeny & Inventory & $\begin{array}{c}\text { Factor } \\
\text { (rem/Ci) }\end{array}$ & Half Life & Kd Source & $\begin{array}{c}\mathrm{Kd} \\
\text { Aquifer } \\
(\mathrm{mL} / \mathrm{g})\end{array}$ & $\begin{array}{c}\text { CEDE } \\
(\mathrm{mrem} / \mathrm{y})\end{array}$ & Peak Time \\
\hline & Ac- 227 & & $1.46 \mathrm{E}+07$ & $2.18 E+01$ & & $1.50 \mathrm{E}+01$ & $9.65 E-08$ & \\
\hline Total & & & & & & & $2.07 \mathrm{E}-02$ & \\
\hline $\mathrm{Pu}-240$ & & 4.70E-02 & $2.29 E+06$ & $6.56 \mathrm{E}+03$ & $5.00 \mathrm{E}+03$ & $1.20 \mathrm{E}+03$ & $4.21 \mathrm{E}-04$ & $1.63 E+04$ \\
\hline & U-236 & & $2.50 \mathrm{E}+05$ & $2.43 E+07$ & & $1.50 \mathrm{E}+01$ & $4.51 E-06$ & \\
\hline & Th-232 & & $2.80 \mathrm{E}+06$ & $1.41 \mathrm{E}+10$ & & $3.30 \mathrm{E}+03$ & 1.19E- 13 & \\
\hline & Ra-228 & & $1.20 \mathrm{E}+06$ & $5.75 \mathrm{E}+00$ & & $3.60 \mathrm{E}+04$ & $4.69 E-15$ & \\
\hline & Th-228 & & $7.50 \mathrm{E}+05$ & $1.91 E+00$ & & $3.30 \mathrm{E}+03$ & $3.19 \mathrm{E}-14$ & \\
\hline Total & & & & & & & $4.26 \mathrm{E}-04$ & \\
\hline $\mathrm{Pu}-241$ & & $2.63 E+02$ & $8.20 E+04$ & $1.44 \mathrm{E}+01$ & $5.00 \mathrm{E}+03$ & $1.20 \mathrm{E}+03$ & $0.00 E+00$ & $0.00 \mathrm{E}+00$ \\
\hline & Am-241 & & $4.50 \mathrm{E}+06$ & $4.32 \mathrm{E}+02$ & & $5.00 \mathrm{E}+03$ & $0.00 E+00$ & \\
\hline & $\mathrm{Np}-237$ & & $3.90 \mathrm{E}+06$ & $2.10 \mathrm{E}+06$ & & $2.50 \mathrm{E}+0 \mathrm{I}$ & $0.00 \mathrm{E}+00$ & \\
\hline & U-233 & & $2.70 E+05$ & $1.59 E+05$ & & $1.50 \mathrm{E}+01$ & $0.00 \mathrm{E}+00$ & \\
\hline & Th-229 & & $3.91 E+06$ & $7.34 \mathrm{E}+03$ & & $3.30 \mathrm{E}+03$ & $0.00 E+00$ & \\
\hline Total & & & & & & & $0.00 \mathrm{E}+00$ & \\
\hline $\mathrm{Ra}-226$ & & $7.40 \mathrm{E}-03$ & $1.10 \mathrm{E}+06$ & $1.60 \mathrm{E}+03$ & $1.00 \mathrm{E}+02$ & $3.60 \mathrm{E}+04$ & $7.06 \mathrm{E}-18$ & $3.80 \mathrm{E}+04$ \\
\hline & $\mathrm{Pb}-210$ & & $6.70 \mathrm{E}+06$ & $2.23 E+01$ & & $1.60 \mathrm{E}+04$ & $9.81 \mathrm{E}-17$ & \\
\hline Total & & & & & & & $1.05 \mathrm{E}-16$ & \\
\hline $\mathrm{Sb}-125$ & & $9.95 \mathrm{E}+01$ & $2.60 \mathrm{E}+03$ & $2.73 E+00$ & $0.00 \mathrm{E}+00$ & $1.50 \mathrm{E}+02$ & $8.93 E-22$ & $9.99 E+01$ \\
\hline $\mathrm{Sr}-90$ & & $1.73 E+02$ & $1.30 \mathrm{E}+05$ & $2.91 \mathrm{E}+01$ & $3.00 \mathrm{E}+00$ & $2.00 \mathrm{E}+01$ & $3.81 E+00$ & $1.29 \mathrm{E}+02$ \\
\hline Tc-99 & & $1.09 \mathrm{E}-01$ & $1.30 \mathrm{E}+03$ & $2.13 E+05$ & $1.00 \mathrm{E}+00$ & $1.00 \mathrm{E}-01$ & $4.30 \mathrm{E}-02$ & $1.60 \mathrm{E}+01$ \\
\hline Th-228 & & 7.37E-04 & $7.50 \mathrm{E}+05$ & $1.91 \mathrm{E}+00$ & $3.30 \mathrm{E}+03$ & $3.30 \mathrm{E}+03$ & $0.00 \mathrm{E}+00$ & $0.00 \mathrm{E}+00$ \\
\hline Th-230 & & $3.98 \mathrm{E}-03$ & $5.30 \mathrm{E}+05$ & $7.54 \mathrm{E}+03$ & $3.30 \mathrm{E}+03$ & $3.30 \mathrm{E}+03$ & $1.78 \mathrm{E}-06$ & $2.82 E+04$ \\
\hline & $\mathrm{RA}-226$ & & $1.10 \mathrm{E}+06$ & $1.60 \mathrm{E}+03$ & & $3.60 \mathrm{E}+04$ & $4.31 \mathrm{E}-07$ & \\
\hline & PB-210 & & $6.70 E+06$ & $2.23 E+01$ & & $1.60 \mathrm{E}+04$ & $5.92 \mathrm{E}-06$ & \\
\hline Total & & & & & & & $8.13 E-06$ & \\
\hline Th-232 & & $3.78 \mathrm{E}-01$ & $2.80 \mathrm{E}+06$ & $1.41 E+10$ & $3.30 \mathrm{E}+03$ & $3.30 \mathrm{E}+03$ & $6.67 \mathrm{E}-02$ & $1.23 E+05$ \\
\hline & $\mathrm{Ra}-228$ & & $1.20 \mathrm{E}+06$ & $5.75 E+00$ & & $3.60 \mathrm{E}+04$ & $2.62 \mathrm{E}-03$ & \\
\hline & Th-228 & & $7.50 \mathrm{E}+05$ & $1.91 E+00$ & & $3.30 \mathrm{E}+03$ & $1.79 \mathrm{E}-02$ & \\
\hline Total & & & & & & & $8.71 \mathrm{E}-02$ & \\
\hline U-232 & & $2.47 \mathrm{E}-03$ & $1.30 \mathrm{E}+06$ & $7.00 \mathrm{E}+01$ & $1.50 E+03$ & $1.50 \mathrm{E}+01$ & $3.18 \mathrm{E}-05$ & $1.93 E+02$ \\
\hline & Th-228 & & $7.51 \mathrm{E}+05$ & $1.91 E+00$ & & $3.30 \mathrm{E}+03$ & $8.73 \mathrm{E}-08$ & \\
\hline Total & & & & & & & $3.19 \mathrm{E}-05$ & \\
\hline U-233 & & $1.92 \mathrm{E}-01$ & $2.70 \mathrm{E}+05$ & $1.59 E+05$ & $1.50 \mathrm{E}+03$ & $1.50 \mathrm{E}+01$ & $1.74 \mathrm{E}-02$ & $9.43 E+02$ \\
\hline & Th-229 & & $1.00 \mathrm{E}+06$ & $7.34 \mathrm{E}+03$ & & $3.30 \mathrm{E}+03$ & $2.55 \mathrm{E}-05$ & \\
\hline Total & & & & & & & $1.75 \mathrm{E}-02$ & \\
\hline $\mathrm{U}-234$ & & $8.27 \mathrm{E}-02$ & $2.60 \mathrm{E}+05$ & $2.45 E+05$ & $1.50 \mathrm{E}+03$ & $1.50 \mathrm{E}+01$ & $7.24 \mathrm{E}-03$ & $9.46 \mathrm{E}+02$ \\
\hline & TH-230 & & $5.30 E+05$ & $7.54 \mathrm{E}+03$ & & $3.30 \mathrm{E}+03$ & $5.69 \mathrm{E}-06$ & \\
\hline & RA-226 & & $1.10 E+06$ & $1.60 \mathrm{E}+03$ & & $3.60 E+04$ & $1.97 \mathrm{E}-07$ & \\
\hline & PB-210 & & $6.70 E+06$ & $2.23 \mathrm{E}+01$ & & $1.60 \mathrm{E}+04$ & $2.54 \mathrm{E}-06$ & \\
\hline Total & & & & & & & $7.25 \mathrm{E}-03$ & \\
\hline $\mathrm{U}-235$ & & $2.81 \mathrm{E}-03$ & $2.50 \mathrm{E}+05$ & $7.04 \mathrm{E}+11$ & $1.50 \mathrm{E}+03$ & $1.50 \mathrm{E}+01$ & $2.38 \mathrm{E}-04$ & $9.60 \mathrm{E}+02$ \\
\hline & $\mathrm{Pa}-231$ & & $1.10 \mathrm{E}+07$ & $3.28 E+04$ & & $1.50 \mathrm{E}+01$ & 2. $10 \mathrm{E}-04$ & \\
\hline & Ac-227 & & $1.46 \mathrm{E}+07$ & $2.18 \mathrm{E}+01$ & & $1.50 \mathrm{E}+01$ & $2.70 \mathrm{E}-04$ & \\
\hline Total & & & & & & & $7.17 E-04$ & \\
\hline
\end{tabular}




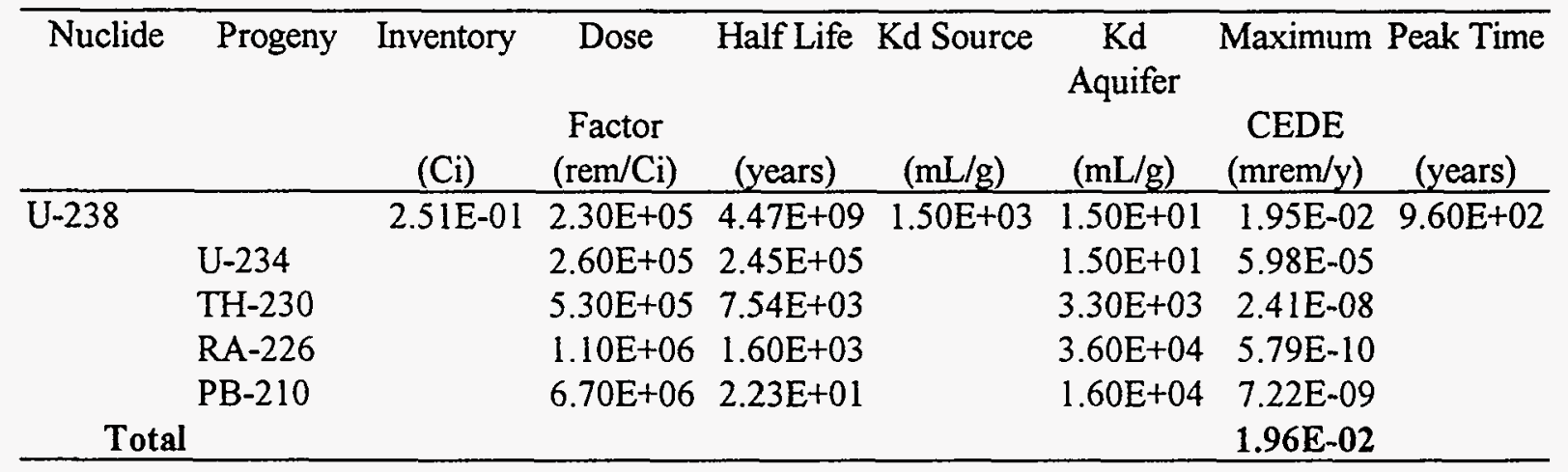

Table 10. Maximum committed effective dose equivalents calculated with GWSCREEN for Class C waste.

\begin{tabular}{|c|c|c|c|c|c|c|c|c|}
\hline \multirow[t]{2}{*}{ Nuclide } & \multirow[t]{2}{*}{ Progeny } & \multirow{2}{*}{$\begin{array}{l}\text { Inventory } \\
\text { (Ci) }\end{array}$} & \multirow{2}{*}{$\begin{array}{l}\text { Dose } \\
\text { Factor } \\
\text { (rem/Ci) }\end{array}$} & \multirow{2}{*}{$\begin{array}{l}\text { Half Life } \\
\text { (years) }\end{array}$} & \multirow{2}{*}{$\begin{array}{l}\text { Kd Source } \\
(\mathrm{mL} / \mathrm{g})\end{array}$} & \multirow{2}{*}{$\begin{array}{c}\mathrm{Kd} \\
\text { Aquifer } \\
(\mathrm{mL} / \mathrm{g})\end{array}$} & \multicolumn{2}{|c|}{ Maximum Peak Time } \\
\hline & & & & & & & $\begin{array}{c}\text { CEDE } \\
(\mathrm{mrem} / \mathrm{y})\end{array}$ & (years) \\
\hline Am-241 & & $1.50 \mathrm{E}-02$ & $4.50 E+06$ & $4.32 E+02$ & $5.00 \mathrm{E}+03$ & $5.00 \mathrm{E}+03$ & $3.16 \mathrm{E}-12$ & $7.61 \mathrm{E}+03$ \\
\hline & $\mathrm{Np}-237$ & & $3.90 \mathrm{E}+06$ & $2.10 \mathrm{E}+06$ & & $2.50 \mathrm{E}+01$ & $2.23 E-08$ & \\
\hline & U-233 & & $2.70 E+05$ & $1.59 E+05$ & & $1.50 \mathrm{E}+01$ & $7.68 \mathrm{E}-11$ & \\
\hline & Th-229 & & $3.91 \mathrm{E}+06$ & $7.34 \mathrm{E}+03$ & & $3.30 \mathrm{E}+03$ & $1.39 \mathrm{E}-12$ & \\
\hline Total & & & & & & & 2.24E-08 & \\
\hline Am-243 & & $1.43 E-05$ & $4.50 E+06$ & $7.38 E+03$ & $5.00 \mathrm{E}+03$ & $5.00 \mathrm{E}+03$ & 4.20E-07 & $3.00 E+04$ \\
\hline & $\mathrm{Pu}-239$ & & $4.30 \mathrm{E}+06$ & $2.41 E+04$ & & $1.20 \mathrm{E}+03$ & $4.48 \mathrm{E}-06$ & \\
\hline & U-235 & & $2.51 E+05$ & $7.04 \mathrm{E}+08$ & & $1.50 \mathrm{E}+01$ & $5.65 \mathrm{E}-10$ & \\
\hline & $\mathrm{Pa}-231$ & & $1.10 \mathrm{E}+07$ & $3.28 E+04$ & & $1.50 \mathrm{E}+01$ & 5.73E-09 & \\
\hline & $\mathrm{Ac}-227$ & & $1.46 \mathrm{E}+07$ & $2.18 \mathrm{E}+01$ & & $1.50 \mathrm{E}+01$ & 7.59E-09 & \\
\hline Total & & & & & & & & \\
\hline $\mathrm{Ba}-133$ & & $1.00 \mathrm{E}-04$ & $3.20 \mathrm{E}+03$ & $1.05 E+01$ & $1.00 \mathrm{E}+01$ & $0.00 \mathrm{E}+00$ & 05 & $3.81 E+00$ \\
\hline C-14 & & $8.84 E+01$ & $2.10 \mathrm{E}+03$ & $5.70 E+03$ & $1.00 \mathrm{E}+02$ & $2.00 \mathrm{E}+01$ & $3.70 E+00$ & $2.98 E+02$ \\
\hline Cd-109 & & $7.63 \mathrm{E}-04$ & $1.20 \mathrm{E}+04$ & $1.27 \mathrm{E}+00$ & $1.00 \mathrm{E}+02$ & $4.00 \mathrm{E}+01$ & $2.77 \mathrm{E}-21$ & $3.62 \mathrm{E}+01$ \\
\hline $\mathrm{Cm}-243$ & & $5.74 \mathrm{E}-03$ & $1.10 E+05$ & $2.85 E+01$ & $1.00 \mathrm{E}+02$ & $1.80 \mathrm{E}+04$ & $0.00 \mathrm{E}+00$ & $0.00 \mathrm{E}+00$ \\
\hline & $\mathrm{Pu}-23$ & & $4.30 E+06$ & $2.41 \mathrm{E}+04$ & & $1.20 \mathrm{E}+03$ & $0.00 E+00$ & \\
\hline & U-235 & & $2.50 \mathrm{E}+05$ & $7.04 \mathrm{E}+11$ & & $1.50 \mathrm{E}+01$ & $0.00 \mathrm{E}+00$ & \\
\hline & $\mathrm{Pa}-231$ & & $1.10 E+07$ & $3.28 E+04$ & & $1.50 \mathrm{E}+01$ & $0.00 \mathrm{E}+00$ & \\
\hline & Ac- -227 & & $1.46 \mathrm{E}+07$ & $2.18 \mathrm{E}+01$ & & $1.50 \mathrm{E}+01$ & $0.00 \mathrm{E}+00$ & \\
\hline Total & & & & & & & 0.00 & \\
\hline $\mathrm{Cm}-244$ & & $1.86 \mathrm{E}-02$ & $2.30 \mathrm{E}+06$ & $1.81 E+01$ & $1.00 \mathrm{E}+02$ & $1.80 E+04$ & $0.00 \mathrm{E}+00$ & $0.00 \mathrm{E}+00$ \\
\hline & $\mathrm{Pu}-240$ & & $2.29 \mathrm{E}+06$ & $6.56 \mathrm{E}+03$ & & $1.20 \mathrm{E}+03$ & $0.00 \mathrm{E}+00$ & \\
\hline & U-236 & & $2.50 \mathrm{E}+05$ & $2.43 E+07$ & & $1.50 \mathrm{E}+01$ & $0.00 \mathrm{E}+00$ & \\
\hline & Th-232 & & $2.80 \mathrm{E}+06$ & $1.41 \mathrm{E}+10$ & & $3.30 \mathrm{E}+03$ & $0.00 \mathrm{E}+00$ & \\
\hline & $\mathrm{Ra}-228$ & & $1.20 \mathrm{E}+06$ & $5.75 E+00$ & & $3.60 \mathrm{E}+04$ & $0.00 \mathrm{E}+00$ & \\
\hline & Th-228 & & $7.50 \mathrm{E}+05$ & $1.91 E+00$ & & $3.30 \mathrm{E}+03$ & $0.00 \mathrm{E}+00$ & \\
\hline Total & & & & & & & $0.00 \mathrm{E}+00$ & \\
\hline Co-60 & & $7.75 \mathrm{E}+05$ & $2.60 E+04$ & $5.75 \mathrm{E}+00$ & $1.00 \mathrm{E}+02$ & $1.30 \mathrm{E}+03$ & $0.00 \mathrm{E}+00$ & $0.00 \mathrm{E}+00$ \\
\hline Cs- 134 & & $5.75 \mathrm{E}+01$ & $7.40 \mathrm{E}+04$ & $2.06 \mathrm{E}+00$ & $2.00 \mathrm{E}+01$ & $4.60 \mathrm{E}+03$ & $0.00 E+00$ & $0.00 \mathrm{E}+00$ \\
\hline Cs-137 & & $4.23 E+02$ & $5.00 \mathrm{E}+04$ & $3.00 \mathrm{E}+01$ & $2.00 \mathrm{E}+01$ & $4.60 \mathrm{E}+03$ & $0.00 \mathrm{E}+00$ & $0.00 \mathrm{E}+00$ \\
\hline
\end{tabular}




\begin{tabular}{|c|c|c|c|c|c|c|c|c|}
\hline \multirow[t]{2}{*}{ Nuclide } & \multirow[t]{2}{*}{ Progeny } & \multirow{2}{*}{$\begin{array}{l}\text { Inventory } \\
\text { (Ci) }\end{array}$} & \multirow{2}{*}{$\begin{array}{c}\text { Dose } \\
\text { Factor } \\
(\mathrm{rem} / \mathrm{Ci})\end{array}$} & \multirow{2}{*}{$\begin{array}{c}\text { Half Life } \\
\text { (years) }\end{array}$} & \multirow{2}{*}{$\begin{array}{l}\text { Kd Source } \\
(\mathrm{mL} / \mathrm{g})\end{array}$} & \multirow{2}{*}{$\begin{array}{c}\mathrm{Kd} \\
\text { Aquifer } \\
(\mathrm{mL} / \mathrm{g})\end{array}$} & \multicolumn{2}{|c|}{ Maximum Peak Time } \\
\hline & & & & & & & $\begin{array}{c}\text { CEDE } \\
(\mathrm{mrem} / \mathrm{y})\end{array}$ & (years) \\
\hline $\mathrm{Fe}-55$ & & $1.03 E+06$ & $5.80 \mathrm{E}+01$ & $2.73 \mathrm{E}+00$ & $1.00 \mathrm{E}+02$ & $8.00 \mathrm{E}+02$ & $0.00 \mathrm{E}+00$ & $0.00 \mathrm{E}+00$ \\
\hline H-3 & & $3.77 \mathrm{E}+03$ & $6.30 \mathrm{E}+01$ & $1.23 E+01$ & $0.00 \mathrm{E}+00$ & $0.00 E+00$ & $1.30 \mathrm{E}+03$ & $2.56 \mathrm{E}+00$ \\
\hline $\mathrm{I}-129$ & & 8.65E-04 & $2.80 E+05$ & $1.57 \mathrm{E}+07$ & $5.00 \mathrm{E}+00$ & $5.00 \mathrm{E}+00$ & $6.08 \mathrm{E}-02$ & $5.91 E+01$ \\
\hline $\mathrm{Nb}-94$ & & $1.58 \mathrm{E}+00$ & $5.10 \mathrm{E}+03$ & $2.00 E+04$ & $1.00 \mathrm{E}+03$ & $5.50 E+02$ & $1.04 \mathrm{E}-02$ & $6.48 E+03$ \\
\hline $\mathrm{Ni}-59$ & & $5.90 \mathrm{E}+02$ & $3.50 E+03$ & $7.50 \mathrm{E}+04$ & $1.00 \mathrm{E}+02$ & $3.00 E+02$ & $1.43 E+01$ & $2.75 E+03$ \\
\hline Ni-63 & & $4.38 E+04$ & $5.40 E+01$ & $1.00 \mathrm{E}+02$ & $1.00 \mathrm{E}+02$ & $3.00 \mathrm{E}+02$ & $9.57 \mathrm{E}-04$ & $9.14 \mathrm{E}+02$ \\
\hline $\mathrm{Np}-237$ & & $7.86 \mathrm{E}-05$ & $3.90 \mathrm{E}+06$ & $2.10 E+06$ & $5.00 \mathrm{E}+03$ & $2.50 \mathrm{E}+01$ & $1.55 \mathrm{E}-04$ & $6.24 E+02$ \\
\hline & U-233 & & $2.70 E+05$ & $1.59 E+05$ & & $1.50 \mathrm{E}+01$ & $4.84 \mathrm{E}-08$ & \\
\hline & Th-229 & & $3.91 E+06$ & $7.34 \mathrm{E}+03$ & & $3.30 \mathrm{E}+03$ & $9.35 \mathrm{E}-11$ & \\
\hline Total & & & & & & & $1.55 \mathrm{E}-04$ & \\
\hline $\mathrm{Pb}-210$ & & $2.00 \mathrm{E}-0 \mathrm{I}$ & $6.70 E+06$ & $2.23 E+01$ & $0.00 \mathrm{E}+00$ & $1.60 \mathrm{E}+04$ & $0.00 \mathrm{E}+00$ & $0.00 \mathrm{E}+00$ \\
\hline $\mathrm{Pu}-238$ & & $1.21 \mathrm{E}-01$ & $3.80 E+06$ & $8.77 \mathrm{E}+01$ & $5.00 \mathrm{E}+03$ & $1.20 \mathrm{E}+03$ & $2.58 \mathrm{E}-12$ & $1.68 E+03$ \\
\hline & U-234 & & $2.60 \mathrm{E}+05$ & $2.45 E+05$ & & $1.50 \mathrm{E}+01$ & $2.77 \mathrm{E}-09$ & \\
\hline & TH-230 & & $5.30 \mathrm{E}+05$ & $7.54 E+03$ & & $3.30 \mathrm{E}+03$ & $3.47 \mathrm{E}-12$ & \\
\hline & RA-226 & & $1.10 \mathrm{E}+06$ & $1.60 \mathrm{E}+03$ & & $3.60 \mathrm{E}+04$ & $1.84 \mathrm{E}-13$ & \\
\hline & PB-210 & & $6.70 \mathrm{E}+06$ & $2.23 \mathrm{E}+01$ & & $1.60 \mathrm{E}+04$ & $2.43 E-12$ & \\
\hline Total & & & & & & & $2.78 \mathrm{E}-09$ & \\
\hline $\mathrm{Pu}-239$ & & $9.70 \mathrm{E}-03$ & $4.30 \mathrm{E}+06$ & $2.41 \mathrm{E}+04$ & $5.00 \mathrm{E}+03$ & $1.20 E+03$ & $1.05 \mathrm{E}-02$ & $1.47 \mathrm{E}+04$ \\
\hline & U-235 & & $2.50 \mathrm{E}+05$ & $7.04 \mathrm{E}+11$ & & $1.50 \mathrm{E}+01$ & $8.62 E-10$ & \\
\hline & $\mathrm{Pa}-23 \mathrm{I}$ & & $1.10 \mathrm{E}+07$ & $3.28 E+04$ & & $1.50 \mathrm{E}+01$ & $5.66 \mathrm{E}-09$ & \\
\hline & Ac-227 & & $1.46 \mathrm{E}+07$ & $2.18 \mathrm{E}+01$ & & $1.50 \mathrm{E}+01$ & $7.48 \mathrm{E}-09$ & \\
\hline Total & & & & & & & $1.05 E-02$ & \\
\hline $\mathrm{Pu}-241$ & & $1.86 \mathrm{E}+01$ & $8.20 E+04$ & $1.44 \mathrm{E}+01$ & $5.00 \mathrm{E}+03$ & $1.20 \mathrm{E}+03$ & $0.00 \mathrm{E}+00$ & $0.00 \mathrm{E}+00$ \\
\hline & Am-241 & & $4.50 \mathrm{E}+06$ & $4.32 \mathrm{E}+02$ & & $5.00 \mathrm{E}+03$ & $0.00 E+00$ & \\
\hline & Np-237 & & $3.90 \mathrm{E}+06$ & $2.10 \mathrm{E}+06$ & & $2.50 E+01$ & $0.00 E+00$ & \\
\hline & U-233 & & $2.70 \mathrm{E}+05$ & $1.59 \mathrm{E}+05$ & & $1.50 \mathrm{E}+01$ & $0.00 \mathrm{E}+00$ & \\
\hline & Th-229 & & $3.91 E+06$ & $7.34 \mathrm{E}+03$ & & $3.30 \mathrm{E}+03$ & $0.00 \mathrm{E}+00$ & \\
\hline Total & & & & & & & $0.00 \mathrm{E}+00$ & \\
\hline & & 7.93E-08 & 4.10E+06 & $8.30 \mathrm{E}+07$ & $5.00 \mathrm{E}+03$ & $1.20 E+03$ & $1.29 \mathrm{E}-07$ & $1.75 E+04$ \\
\hline & U-238 & & $2.30 \mathrm{E}+05$ & $4.47 E+09$ & & $1.50 E+01$ & $1.55 E-12$ & \\
\hline & U-234 & & $2.60 \mathrm{E}+05$ & $2.45 \mathrm{E}+05$ & & $1.50 \mathrm{E}+01$ & $4.28 E-14$ & \\
\hline & TH-230 & & $5.30 \mathrm{E}+05$ & $7.54 \mathrm{E}+03$ & & $3.30 \mathrm{E}+03$ & $1.52 \mathrm{E}-16$ & \\
\hline & RA-226 & & $1.10 \mathrm{E}+06$ & $1.60 \mathrm{E}+03$ & & $3.60 \mathrm{E}+04$ & $2.07 \mathrm{E}-17$ & \\
\hline & PB-210 & & $6.70 E+06$ & $2.23 E+01$ & & $1.60 \mathrm{E}+04$ & $2.82 E-16$ & \\
\hline Total & & & & & & & $1.29 \mathrm{E}-07$ & \\
\hline $\mathrm{Sb}-125$ & & $3.55 E+02$ & $2.60 E+03$ & $2.73 E+00$ & $0.00 \mathrm{E}+00$ & $1.50 E+02$ & $1.75 \mathrm{E}-19$ & $9.89 \mathrm{E}+01$ \\
\hline Sr-90 & & $1.30 \mathrm{E}+00$ & $1.30 \mathrm{E}+05$ & $2.91 \mathrm{E}+01$ & $3.00 \mathrm{E}+00$ & $2.00 \mathrm{E}+01$ & $9.24 \mathrm{E}-01$ & $1.09 \mathrm{E}+02$ \\
\hline Tc-99 & & $5.61 \mathrm{E}-03$ & $1.30 \mathrm{E}+03$ & $2.13 E+05$ & $1.00 \mathrm{E}+00$ & $1.00 \mathrm{E}-01$ & $1.18 \mathrm{E}-02$ & $4.91 E+00$ \\
\hline
\end{tabular}


Table 11. Maximum gross alpha activity in groundwater (excluding radium and radon) for Class AB waste using the GWSCREEN code and screening model assumptions and methodology.

\begin{tabular}{|c|c|c|}
\hline Radionuclide & Progeny & $\begin{array}{l}\text { Maximum } \\
\text { Alpha Activity } \\
(\mathrm{pCi} / \mathrm{L})\end{array}$ \\
\hline \multirow[t]{4}{*}{$\mathrm{Am}-241$} & & $9.86 \mathrm{E}-14$ \\
\hline & $\mathrm{Np}-237$ & $9.39 \mathrm{E}-10$ \\
\hline & U-233 & $4.73 \mathrm{E}-11$ \\
\hline & Th-229 & $5.98 \mathrm{E}-14$ \\
\hline Total & & $9.86 \mathrm{E}-10$ \\
\hline \multirow[t]{4}{*}{$\mathrm{Am}-243$} & & $2.15 \mathrm{E}-09$ \\
\hline & $\mathrm{Pu}-239$ & $3.34 \mathrm{E}-08$ \\
\hline & $\mathrm{U}-235$ & $9.05 \mathrm{E}-11$ \\
\hline & $\mathrm{Pa}-231$ & $2.41 E-11$ \\
\hline Total & & $3.56 \mathrm{E}-08$ \\
\hline \multirow[t]{4}{*}{$\mathrm{Cm}-243$} & & $0.00 \mathrm{E}+00$ \\
\hline & $\mathrm{Pu}-239$ & $0.00 \mathrm{E}+00$ \\
\hline & U-235 & $0.00 \mathrm{E}+00$ \\
\hline & $\mathrm{Pa}-231$ & $0.00 \mathrm{E}+00$ \\
\hline Total & & $0.00 \mathrm{E}+00$ \\
\hline \multirow[t]{5}{*}{$\mathrm{Cm}-244$} & & $0.00 \mathrm{E}+00$ \\
\hline & $\mathrm{Pu}-240$ & $0.00 \mathrm{E}+00$ \\
\hline & U-236 & $0.00 E+00$ \\
\hline & Th-232 & $0.00 \mathrm{E}+00$ \\
\hline & Th-228 & $0.00 \mathrm{E}+00$ \\
\hline Total & & $0.00 \mathrm{E}+00$ \\
\hline \multirow[t]{3}{*}{$\mathrm{Pu}-238$} & & $2.80 \mathrm{E}-14$ \\
\hline & U-234 & $5.05 E-10$ \\
\hline & TH-230 & $3.13 E-13$ \\
\hline Total & & $5.05 \mathrm{E}-10$ \\
\hline \multirow[t]{3}{*}{ Pu-239 } & & $6.59 \mathrm{E}-03$ \\
\hline & $\mathrm{U}-235$ & $2.83 \mathrm{E}-08$ \\
\hline & $\mathrm{Pa}-231$ & $9.06 \mathrm{E}-09$ \\
\hline Total & & $6.59 \mathrm{E}-03$ \\
\hline \multirow[t]{4}{*}{ Pu-240 } & & $2.52 \mathrm{E}-04$ \\
\hline & U-236 & $2.47 \mathrm{E}-05$ \\
\hline & Th-232 & $5.84 \mathrm{E}-14$ \\
\hline & Th-228 & $5.83 E-14$ \\
\hline Total & & $2.77 \mathrm{E}-04$ \\
\hline Pu-241 & Am-241 & $\begin{array}{l}0.00 \mathrm{E}+00 \\
0.00 \mathrm{E}+00\end{array}$ \\
\hline
\end{tabular}




\begin{tabular}{|c|c|c|}
\hline Radionuclide & Progeny & $\begin{array}{c}\text { Maximum } \\
\text { Alpha Activity } \\
(\mathrm{pCi} / \mathrm{L})\end{array}$ \\
\hline & $\mathrm{Np}-237$ & $0.00 \mathrm{E}+00$ \\
\hline & U-233 & $0.00 \mathrm{E}+00$ \\
\hline & Th-229 & $0.00 \mathrm{E}+00$ \\
\hline Total & & $0.00 \mathrm{E}+00$ \\
\hline Th-228 & & $0.00 \mathrm{E}+00$ \\
\hline Th-230 & & $4.61 E-06$ \\
\hline Th-232 & & $3.26 \mathrm{E}-02$ \\
\hline & Th-228 & $3.26 \mathrm{E}-02$ \\
\hline Total & & $6.52 \mathrm{E}-02$ \\
\hline$U-232$ & & $3.35 \mathrm{E}-05$ \\
\hline & Th-228 & $1.59 \mathrm{E}-07$ \\
\hline Total & & $3.37 \mathrm{E}-05$ \\
\hline U-233 & & $8.85 \mathrm{E}-02$ \\
\hline & Th-229 & $3.49 \mathrm{E}-05$ \\
\hline Total & & $8.85 \mathrm{E}-02$ \\
\hline U-234 & & $3.82 \mathrm{E}-02$ \\
\hline & TH-230 & $1.47 \mathrm{E}-05$ \\
\hline Total & & $3.82 \mathrm{E}-02$ \\
\hline U-235 & & $1.30 \mathrm{E}-03$ \\
\hline & $\mathrm{Pa}-231$ & $2.61 \mathrm{E}-05$ \\
\hline Total & & $1.33 \mathrm{E}-03$ \\
\hline U-238 & & $1.16 \mathrm{E}-01$ \\
\hline & U-234 & $3.15 \mathrm{E}-04$ \\
\hline & TH-230 & $6.24 \mathrm{E}-08$ \\
\hline Total & & $1.16 \mathrm{E}-01$ \\
\hline
\end{tabular}

Table 12. Maximum gross alpha activity in groundwater (excluding radium and radon) for Class $C$ waste using the GWSCREEN code and screening model assumptions and methodology.

\begin{tabular}{llc}
\hline Radionuclide & Progeny & $\begin{array}{c}\text { Maximum } \\
\text { Alpha Activity } \\
(\mathrm{pCi} / \mathrm{L})\end{array}$ \\
\hline Am-241 & $\mathrm{Np}-237$ & $9.62 \mathrm{E}-13$ \\
& $\mathrm{U}-233$ & $7.84 \mathrm{E}-09$ \\
& $\mathrm{Th}-229$ & $3.90 \mathrm{E}-10$ \\
Total & & $4.87 \mathrm{E}-13$ \\
& & $8.23 \mathrm{E}-09$
\end{tabular}




\begin{tabular}{|c|c|c|}
\hline Radionuclide & Progeny & $\begin{array}{c}\text { Maximum } \\
\text { Alpha Activity } \\
(\mathrm{pCi} / \mathrm{L})\end{array}$ \\
\hline \multirow[t]{5}{*}{ Am-243 } & & $1.28 \mathrm{E}-07$ \\
\hline & $\mathrm{Pu}-239$ & $1.43 \mathrm{E}-06$ \\
\hline & U-235 & $3.09 \mathrm{E}-09$ \\
\hline & $\mathrm{Pa}-231$ & $7.13 \mathrm{E}-10$ \\
\hline & Ac- 227 & 7.12E-10 \\
\hline Total & & $1.56 \mathrm{E}-06$ \\
\hline \multirow[t]{5}{*}{$\mathrm{Cm}-243$} & & $0.00 \mathrm{E}+00$ \\
\hline & $\mathrm{Pu}-239$ & $0.00 \mathrm{E}+00$ \\
\hline & $\mathrm{U}-235$ & $0.00 \mathrm{E}+00$ \\
\hline & $\mathrm{Pa}-231$ & $0.00 \mathrm{E}+00$ \\
\hline & Ac-227 & $0.00 \mathrm{E}+00$ \\
\hline Total & & $0.00 \mathrm{E}+00$ \\
\hline \multirow[t]{6}{*}{$\mathrm{Cm}-244$} & & $0.00 \mathrm{E}+00$ \\
\hline & $\mathrm{Pu}-240$ & $0.00 \mathrm{E}+00$ \\
\hline & U-236 & $0.00 \mathrm{E}+00$ \\
\hline & Th-232 & $0.00 \mathrm{E}+00$ \\
\hline & $\mathrm{Ra}-228$ & $0.00 \mathrm{E}+00$ \\
\hline & Th-228 & $0.00 \mathrm{E}+00$ \\
\hline Total & & $0.00 \mathrm{E}+00$ \\
\hline \multirow[t]{3}{*}{ Np-237 } & & $5.46 \mathrm{E}-05$ \\
\hline & U-233 & $2.45 \mathrm{E}-07$ \\
\hline & Th-229 & $3.28 \mathrm{E}-11$ \\
\hline Total & & $5.48 \mathrm{E}-05$ \\
\hline \multirow[t]{5}{*}{ Pu-238 } & & $9.30 \mathrm{E}-13$ \\
\hline & U-234 & $1.46 \mathrm{E}-08$ \\
\hline & TH-230 & $8.97 \mathrm{E}-12$ \\
\hline & $\mathrm{RA}-226$ & $2.29 \mathrm{E}-13$ \\
\hline & PB-210 & $4.97 \mathrm{E}-13$ \\
\hline Total & & i. $46 \mathrm{E}-08$ \\
\hline \multirow[t]{4}{*}{ Pu-239 } & & $3.34 \mathrm{E}-03$ \\
\hline & U-235 & 4.72E-09 \\
\hline & $\mathrm{Pa}-231$ & $7.05 \mathrm{E}-10$ \\
\hline & Ac- 227 & $7.02 \mathrm{E}-10$ \\
\hline Total & & $3.34 \mathrm{E}-03$ \\
\hline \multirow[t]{4}{*}{$\mathrm{Pu}-241$} & & $0.00 \mathrm{E}+00$ \\
\hline & Am-241 & $0.00 \mathrm{E}+00$ \\
\hline & $\mathrm{Np}-237$ & $0.00 \mathrm{E}+00$ \\
\hline & U-233 & $0.00 \mathrm{E}+00$ \\
\hline
\end{tabular}




\begin{tabular}{llc}
\hline Radionuclide & Progeny & $\begin{array}{c}\text { Maximum } \\
\text { Alpha Activity } \\
(\mathrm{pCi} / \mathrm{L})\end{array}$ \\
\hline \multirow{3}{*}{ Total } & Th-229 & $0.00 \mathrm{E}+00$ \\
& & $0.00 \mathrm{E}+00$ \\
Pu-242 & & \\
& & $4.32 \mathrm{E}-08$ \\
& $\mathrm{U}-238$ & $9.24 \mathrm{E}-12$ \\
& $\mathrm{U}-234$ & $2.25 \mathrm{E}-13$ \\
& TH-230 & $3.92 \mathrm{E}-16$ \\
Total & RA-226 & $2.58 \mathrm{E}-17$ \\
& PB-210 & $5.77 \mathrm{E}-17$ \\
& & $4.32 \mathrm{E}-08$ \\
\hline
\end{tabular}

The PORFLO model was employed to better simulate the actual performance of the facility for ${ }^{3} \mathrm{H}$ and ${ }^{14} \mathrm{C}$. Waste grouting, engineered barriers, and cover emplacement were accounted for in the PORFLO simulation. These simulations were performed early in the project for inventory and sorption coefficient estimates that were different from those received as the "best estimate values" in May, 1996 (see Appendix A). The initial inventory estimate did not include ${ }^{14} \mathrm{C}$ and the sorption coefficients assigned to each element were different than were in the best estimate. Tritium was included in the initial inventory estimate and a $\mathrm{K}_{d}$ value of 0 was assumed in all cases. Therefore, the ${ }^{3} \mathrm{H}$ dose needed only to be adjusted for the final inventory. Because ${ }^{14} \mathrm{C}$ was not included in the initial inventory estimate, a "surrogate" nuclide that had similar sorptive properties was selected to be used in its place. The dispersive behavior of the surrogate nuclide would then mimic that ${ }^{14}{ }^{14} \mathrm{C}$ thereby providing a means to estimate the ${ }^{14} \mathrm{C}$ concentration in groundwater. Once a ${ }^{14} \mathrm{C}$ groundwater concentration was estimated, the CEDE for ${ }^{14} \mathrm{C}$ could be determined. Uranium was investigated as a possible surrogate. The uranium sorption coefficient used in the initial analysis was $100 \mathrm{~mL} / \mathrm{g}$ for concrete and $0.03 \mathrm{~mL} / \mathrm{g}$ for loam. The carbon $\mathrm{K}_{d}$ values used in this analysis were $100 \mathrm{~mL} / \mathrm{g}$ for concrete and $20 \mathrm{~mL} / \mathrm{g}$ for loam. A higher $K_{d}$ value has the effect of reducing the aqueous phase concentration. Therefore, using uranium as the surrogate contaminant with the previously stated $K_{d}$ values will result in overestimates of the ${ }^{14} \mathrm{C}$ concentration in groundwater because carbon has a higher sorption coefficient in loam (20 $\mathrm{mL} / \mathrm{g}$ compared to $0.03 \mathrm{~mL} / \mathrm{g}$ ). The ${ }^{234} \mathrm{U}$ isotope was used as the surrogate because it was represented in Class $A B$ and $C$ waste. Corrections were made for the different nuclide specific decay rates.

For tritium, the dose based on the best estimate inventory is given by

$$
\mathrm{D}=\mathrm{D}_{\text {old }} \times \mathrm{I}_{\text {new }} \mathrm{I}_{\text {old }}
$$

where

$$
\begin{aligned}
& \mathrm{D}=\text { radiological dose (50-year committed effective dose equivalent, mrem) for best estimate } \\
& \quad \text { inventory } \\
& \mathrm{D}_{\text {old }}=\text { dose calculated using initial inventory estimate }(\mathrm{mrem}) \\
& \mathrm{I}_{\text {new }}=\text { best estimate inventory }(\mathrm{Ci}) \\
& \mathrm{I}_{\text {old }}=\text { initial inventory }(\mathrm{Ci}) .
\end{aligned}
$$

For Class $\mathrm{AB}$ waste, the ${ }^{3} \mathrm{H}$ dose calculated from the PORFLO generated groundwater concentrations and the initial inventory of $4,650 \mathrm{Ci}$ was $1.3 \times 10^{-10}$ mrem. The best estimate ${ }^{3} \mathrm{H}$ inventory was $353,309 \mathrm{Ci}$. The best estimate ${ }^{3} \mathrm{H}$ dose is then $1.3 \times 10^{-10} \mathrm{mrem} \times 353,309 \mathrm{Ci} / 4,650 \mathrm{Ci}$ 
$=9.9 \times 10^{-9} \mathrm{mrem}$. For Class $\mathrm{C}$ waste, the dose calculated from PORFLO generated groundwater concentrations and an inventory of $7 \mathrm{Ci}$ was $9.8 \times 10^{-13} \mathrm{mrem}$. The best estimate inventory was 3,770 $\mathrm{Ci}$. Therefore, the ${ }^{3} \mathrm{H}$ dose for Class $\mathrm{C}$ waste is $9.8 \times 10^{-13} \mathrm{mrem} \times 3,770 \mathrm{Ci} / 7 \mathrm{Ci}=5.2 \times 10^{-10} \mathrm{mrem}$. These doses are notably lower than the $4 \mathrm{mrem} / \mathrm{y}$ dose limit.

For the ${ }^{14} \mathrm{C}$ dose calculation, the following equation was used to estimate the dose:

$$
\mathrm{D}=\mathrm{D}_{\text {old }} \times \mathrm{DCF}_{\mathrm{C}-14} \times \mathrm{I}_{\mathrm{C}-14} \times \mathrm{DC} /\left(\mathrm{DCF}_{\mathrm{U}-234} \mathrm{I}_{\mathrm{U}-234}\right)
$$

where

$\mathrm{D}=$ radiological dose (50-year committed effective dose equivalent, mrem) for best estimate inventory

$D_{\text {old }}=$ dose calculated for U-234 using initial inventory (mrem)

$\mathrm{DCF}_{\mathrm{U}-234}=$ dose conversion factor for ${ }^{234} \mathrm{U}(\mathrm{mrem} / \mathrm{Ci})$

$\mathrm{DCF}_{\mathrm{C}-14}=$ dose conversion factor for ${ }^{14} \mathrm{C}(\mathrm{mrem} / \mathrm{Ci})$

$\mathrm{I}_{\mathrm{U}-234}=$ initial inventory for ${ }^{234} \mathrm{U}(\mathrm{Ci})$

$\mathrm{I}_{\mathrm{C}-14}={ }^{14} \mathrm{C}$ inventory $(\mathrm{Ci})$

$\mathrm{DC}=$ decay correction

The decay correction term (DC) is the ratio of the term, $e^{\lambda t}$ for ${ }^{234} \mathrm{U}$ and ${ }^{14} \mathrm{C}\left({ }^{23-4} \mathrm{U} /{ }^{14} \mathrm{C}\right)$ where $\lambda=$ the decay rate constant and $t=$ the time to maximum (peak) concentration for ${ }^{234} U$.

Estimates of the maximum ${ }^{14} \mathrm{C}$ dose using ${ }^{234} \mathrm{U}$ as a surrogate and Equation 6 were $0.77 \mathrm{mrem} / \mathrm{y}$ for Class $\mathrm{AB}$ waste and $0.016 \mathrm{mrem} / \mathrm{y}$ for Class $\mathrm{C}$ waste (Table 13). The estimated doses for ${ }^{14} \mathrm{C}$ and ${ }^{3} \mathrm{H}$ are all below the $4 \mathrm{mrem} / \mathrm{y}$ EDE groundwater dose criteria for fission and activation products..

Table 13. Initial and best estimate inventory, peak times, and maximum drinking water doses for ${ }^{234} U$ and ${ }^{14} \mathrm{C}$, Class $\mathrm{AB}$ and $\mathrm{C}$ waste.

\begin{tabular}{lllll}
\hline & Class AB & Class AB & Class C & Class C \\
& $\mathrm{C}-14$ & $\mathrm{U}-234$ & $\mathrm{C}-14$ & $\mathrm{U}-234$ \\
\hline Dose Conversion Factor (mrem/Ci) & $2.1 \mathrm{E}+06$ & $2.8 \mathrm{E}+08$ & $2.1 \mathrm{E}+06$ & $2.8 \mathrm{E}+08$ \\
Initial Inventory Estimate $(\mathrm{Ci})$ & none & $3.96 \mathrm{E}-01$ & none & $3.96 \mathrm{E}-01$ \\
Best Estimate Inventory $(\mathrm{Ci})$ & 231 & $\mathrm{n} / \mathrm{a}$ & $3.77 \mathrm{E}+03$ & $\mathrm{n} / \mathrm{a}$ \\
Initial ${ }^{234} \mathrm{U}$ Dose Estimate (mrem) & $\mathrm{n} / \mathrm{a}$ & 0.19 & $\mathrm{n} / \mathrm{a}$ & 0.01 \\
Peak Time (years) & $\mathrm{n} / \mathrm{a}$ & $1.3 \mathrm{E}+03$ & $\mathrm{n} / \mathrm{a}$ & $1.18 \mathrm{E}+03$ \\
Maximum ${ }^{14} \mathrm{C}$ dose (mrem) & 0.77 & $\mathrm{n} / \mathrm{a}$ & 0.016 & $\mathrm{n} / \mathrm{a}$ \\
\hline $\mathrm{n} / \mathrm{a}=$ not applicable & & & & \\
\hline
\end{tabular}

Note that the ratio of the ${ }^{3} \mathrm{H}$ dose based on the PORFLO model to that based on the GWSCREEN model was much greater than the similar ratio for ${ }^{14} \mathrm{C}$. For example, the ${ }^{3} \mathrm{H}$ dose for Class $\mathrm{AB}$ waste and the GWSCREEN model was $1.4 \times 10^{4} \mathrm{mrem}$. The dose using groundwater concentrations calculated with PORFLO was $9.9 \times 10^{-9}$ mrem. The ratio of the two doses is $1.4 \times 10^{12}$. The dose ratio for Class $A B{ }^{14} \mathrm{C}$ was $1.7 \mathrm{mrem} / 0.77 \mathrm{mrem}=2.2$. This difference is due to two factors:

- Tritium has a much shorter half-life than ${ }^{14} \mathrm{C}\left(12.3\right.$ years to 5740 year for $\left.{ }^{14} \mathrm{C}\right)$ Therefore, waste containment increases hold-up time and allows more of the nuclide to decay before coming in contact with water.

- The ${ }^{14} \mathrm{C}$ groundwater concentrations calculated with PORFLO used a $\mathrm{K}_{d}$ value of $0.03 \mathrm{~mL} / \mathrm{g}$ for the aquifer (loam) compared to $20 \mathrm{~mL} / \mathrm{g}$ used in the GWSCREEN analysis. 
Accounting for the waste facility's engineered barriers and containment structures have a greater effect on doses estimated for short lived nuclides because a greater fraction of the activity decays before water comes in contact with the waste material and leaching begins. For longer lived nuclides (half life $>1,000$ years ), engineered barriers are not as effective in reducing estimated doses. Higher sorption coefficients in the waste form have a greater impact on reducing doses than engineered barriers for long half-lived nuclides because the nuclide leach rates are reduced both before and after failure of the containment system.

\section{REFERENCES}

Allard, B., 1985. Radionuclide Sorption on Concrete. Nationale Genossenschaft fur die Langerung Radioaktiver Abfalle, NAGRA-NTB-85-21, November.

ANSI/ANS-10.4, 1987, "American National Standard Guidelines for the Verification and Validation of Scientific and Engineering Programs for the Nuclear Industry."

Campbell, G. S., 1985, Soil Physics with Basic, Elsevier, New York.

Carsel, R. F. and R. S. Parrish, 1988, "Developing Joint Probability Distributions of Soil Water Retention Characteristics," Water Resources Research, Vol. 24, pp. 755-769.

Grove Engineering, 1992, MicroShield, Version 4.0, User's Manual, by C. A. Negin and G. Worku.

International Atomic Energy Agency, 1995, Specification for Test Case 2c, Coordinated Research Programme on "The Safety Assessment of Near Surface Radioactive Waste Disposal Facilities (NSARS)."

Kanipe, F. L. and K. S. Neuhauser, 1992, RADTRAN 4:User Guide, Volume 3, NTIS 1990 (\#22-24)1995(\#1-12), Sandia National Laboratory, January.

Napier, B. A., D. L. Strenge, R. A. Peloquin, and J. V. Ramsdell, 1988, GENII- The Hanford Environmental Radiation Dosimetry Software System, PNL-6584, Pacific Northwest Laboratory, December.

Neuhauser and Knipe, 1992 (RadTran?)

Oztunali and Roles, 1986, Update of Part 61 Impacts Analysis Methodology, NUREG/CR-4370.

Rood, A. S., 1994, GWSCREEN: A Semi-Analytical Model for the Assessment of the Groundwater Pathway from Surface or Buried Contamination: Theory and User's Manual, EGG-GEO10158, Revision 2, Idaho National Engineering Laboratory, June.

Runchal, A. K. and B. Sagar, 1991, PORFLOW-A Model for Fluid Flow. Heat and Mass transport in 
Multifluid, Multiphase, Fractured or Porous Media, User's Manual, Version 2.39, ACRi/016/Rev. F, Analytical and Computational Research, Inc., Los Angeles, CA, June.

Sheppard, M. I. and D. H. Thibault, 1990. "Default Soil/Liquid Partition Coefficients (Kds, for Four Major Soil Types: A Compendium". Health Physics, 59:4 pp471-482.

Smith, C. S. and Whitker, C. A., 1993, Independent Verification and Limited Benchmarking Testing of the GWSCREEN Computed Code, EGG-GEO-0799, Idaho National Engineering Laboratory.

van Genuchten, M. Th., 1978, Calculation the Unsaturated Hydraulic Conductivity with a New Closed-Form Analytical Model, 78-WR-08, Water Resources Program, Department of Civil Engineering, Princeton University, Princeton, NJ.

Walton, J. C., L. E. Plansky, and R. W. Smith, 1990, Models for Estimation of Service Life of Concrete Barriers in Low-Level Radioactive Waste Disposal, NUREG/CR-5542, U.S. Nuclear Regulatory Commission.

Waters, R. 1996. PerformanceEvaluation of the Technical Capabilities of DOE Sites for Disposal of Mixed Low-Level Waste: Technical Basis and Discussion of Results. DOE-ID-10521, Volume 2, March.

Wenzel, D. R., 1995, The Radiological Safety Analysis Computer Program (RSAC-5) User's Manual, WINCO-1 123, Rev. 1, Idaho National Engineering Laboratory, February.

Yu, A. D., C. A. Langton, and M. G. Serrato, 1993, "Physical Properties Measurements Program," WSRC-93-894, Westinghouse Savannah River Company, Aiken, SC. 


\section{Appendix A}

\section{Best Estimate Inventory for Class $A B$ and $C$ Waste}

Best estimate inventories for Class $A B$ and $C$ waste were received from W. P. Dornsife of the Pennsylvania Department of Environmental Resources on May 20, 1996. Nuclides were prescreened for the groundwater pathway based on the half-life (see the last column in Tables A-1 and A-2). If the half-life was less than one year, then the nuclide was eliminated from the groundwater pathway analysis.

Table A-1 Best estimate inventories for Class AB waste. The 30 year total is 10 times the "Total $A+B$ " column and was used in the groundwater analysis.

\begin{tabular}{|c|c|c|c|c|c|c|c|}
\hline Nuclide & Half-Life & & $\begin{array}{c}\text { Class A } \\
(\mathrm{mCi}) \\
\end{array}$ & $\begin{array}{c}\text { Class B } \\
(\mathrm{mCi}) \\
\end{array}$ & $\begin{array}{c}\text { Total } A+B \\
(m C i)\end{array}$ & $\begin{array}{c}30 \text { year total } \\
(\mathrm{mCi})\end{array}$ & $\begin{array}{c}\text { Pre- } \\
\text { Screened? }\end{array}$ \\
\hline AG-110M & $2.50 \mathrm{E}+02$ & $\mathrm{~d}$ & $1.06 \mathrm{E}+04$ & $6.80 \mathrm{E}+04$ & $7.86 \mathrm{E}+04$ & $7.86 \mathrm{E}+05$ & yes \\
\hline AM-241 & $4.32 E+02$ & $\mathrm{yr}$ & $9.72 \mathrm{E}+00$ & $3.19 \mathrm{E}+00$ & $1.29 \mathrm{E}+01$ & $1.29 E+02$ & \\
\hline AM-243 & $7.38 \mathrm{E}+03$ & $\mathrm{yr}$ & $1.00 \mathrm{E}-03$ & & $1.00 \mathrm{E}-03$ & $1.00 \mathrm{E}-02$ & \\
\hline $\mathrm{BA}-133$ & $1.05 E+01$ & $\mathrm{yr}$ & $1.85 \mathrm{E}+00$ & 4.29E-03 & $1.85 E+00$ & $1.85 E+01$ & \\
\hline BA-140 & $1.28 \mathrm{E}+01$ & $\mathrm{~d}$ & $3.88 E+04$ & $3.33 E+03$ & $4.21 \mathrm{E}+04$ & $4.21 E+05$ & yes \\
\hline BE-7 & $5.34 \mathrm{E}+01$ & d & $1.00 E+03$ & & $1.00 \mathrm{E}+03$ & $1.00 E+04$ & yes \\
\hline C-14 & $5.73 E+03$ & yr & $2.03 E+04$ & $2.79 E+03$ & $2.31 \mathrm{E}+04$ & $2.31 E+05$ & \\
\hline $\mathrm{CA}-45$ & $1.63 E+02$ & $\mathrm{~d}$ & $3.17 \mathrm{E}+02$ & & $3.17 \mathrm{E}+02$ & $3.17 \mathrm{E}+03$ & yes \\
\hline CD-109 & $1.27 \mathrm{E}+00$ & yr & $4.25 E+00$ & $1.32 \mathrm{E}-02$ & $4.26 E+00$ & $4.26 E+01$ & \\
\hline CE-139 & $1.38 \mathrm{E}+02$ & $\mathrm{~d}$ & $6.42 \mathrm{E}-01$ & & $6.42 \mathrm{E}-01$ & $6.42 \mathrm{E}+00$ & yes \\
\hline CE-14I & $3.25 \mathrm{E}+01$ & d & $3.43 E+03$ & $3.47 \mathrm{E}+02$ & $3.78 \mathrm{E}+03$ & $3.78 E+04$ & yes \\
\hline CE-144 & $2.84 \mathrm{E}+02$ & $\mathrm{~d}$ & $1.78 \mathrm{E}+03$ & $2.70 E+02$ & $2.05 E+03$ & $2.05 E+04$ & yes \\
\hline CF-252 & $2.64 \mathrm{E}+00$ & $\mathrm{yr}$ & $1.00 \mathrm{E}-03$ & & $1.00 \mathrm{E}-03$ & $1.00 \mathrm{E}-02$ & \\
\hline CL-36 & $3.01 E+05$ & yr & $1.31 E+01$ & & $1.31 E+01$ & $1.31 \mathrm{E}+02$ & \\
\hline CM-242 & $1.63 E+02$ & $\mathrm{~d}$ & $2.86 E+01$ & $3.82 E+01$ & $6.67 E+01$ & $6.67 E+02$ & yes \\
\hline CM-243 & $2.85 E+01$ & yr & $3.10 \mathrm{E}+00$ & $2.12 \mathrm{E}-01$ & $3.32 E+00$ & $3.32 E+01$ & \\
\hline CM-244 & $1.81 E+01$ & yr & $7.41 \mathrm{E}+00$ & $5.82 \mathrm{E}+00$ & $1.32 \mathrm{E}+01$ & $1.32 \mathrm{E}+02$ & \\
\hline $\mathrm{CO}-57$ & $2.71 \mathrm{E}+02$ & $d$ & $4.27 E+02$ & $4.45 E+03$ & $4.88 E+03$ & $4.88 E+04$ & yes \\
\hline $\mathrm{CO}-58$ & $7.08 \mathrm{E}+01$ & $d$ & $2.37 E+05$ & $1.02 E+06$ & $1.26 E+06$ & $1.26 E+07$ & yes \\
\hline $\mathrm{CO}-60$ & $5.27 \mathrm{E}+00$ & $y \mathrm{r}$ & $1.76 \mathrm{E}+06$ & $2.97 \mathrm{E}+06$ & $4.73 E+06$ & $4.73 E+07$ & \\
\hline CR-5 I & $2.77 \mathrm{E}+01$ & $\mathrm{~d}$ & $8.16 \mathrm{E}+05$ & $1.40 \mathrm{E}+06$ & $2.21 E+06$ & $2.21 E \div 07$ & yes \\
\hline CS-134 & $2.06 \mathrm{E}+00$ & yr & $1.76 \mathrm{E}+05$ & $5.87 E+05$ & $7.63 \mathrm{E}+05$ & $7.63 \mathrm{E}+06$ & \\
\hline CS-137 & $3.00 \mathrm{E}+01$ & $\mathrm{yT}$ & $4.09 E+05$ & $8.56 \mathrm{E}+05$ & $1.27 E+06$ & $1.27 E+07$ & \\
\hline EU-152 & $1.36 \mathrm{E}+01$ & $y r$ & $2.53 E+01$ & & $2.53 \mathrm{E}+01$ & $2.53 \mathrm{E}+02$ & \\
\hline EU-154 & $8.60 E+00$ & $\mathrm{yr}$ & $1.15 \mathrm{E}-01$ & & $1.15 \mathrm{E}-01$ & $1.15 E+00$ & \\
\hline EU-155 & $4.96 \mathrm{E}+00$ & $y r$ & $1.61 \mathrm{E}-01$ & & $1.61 \mathrm{E}-01$ & $1.61 E+00$ & \\
\hline FE-55 & $2.70 \mathrm{E}+00$ & $y \mathrm{~T}$ & $5.20 E+06$ & $1.19 \mathrm{E}+07$ & $1.71 \mathrm{E}+07$ & $1.71 E+08$ & \\
\hline FE-59 & $4.46 \mathrm{E}+01$ & $\mathrm{~d}$ & $1.23 E+05$ & $5.26 E+04$ & $1.76 E+05$ & $1.76 E+06$ & yes \\
\hline GD-153 & $2.42 E+02$ & $d$ & $1.91 E+02$ & $2.91 \mathrm{E}+02$ & $4.83 E+02$ & $4.83 E+03$ & yes \\
\hline GE-68 & $2.71 \mathrm{E}+02$ & $d$ & $2.41 E-01$ & & $2.41 \mathrm{E}-01$ & $2.41 E+00$ & yes \\
\hline $\mathrm{H}-3$ & $1.23 \mathrm{E}+01$ & yr & $3.11 E+06$ & $3.22 E+07$ & $3.53 \mathrm{E}+07$ & $3.53 E+08$ & \\
\hline HF-181 & $4.24 \mathrm{E}+01$ & $\mathrm{~d}$ & $5.07 \mathrm{E}+02$ & & $5.07 \mathrm{E}+02$ & $5.07 \mathrm{E}+03$ & yes \\
\hline I- 125 & $6.01 \mathrm{E}+01$ & d & $1.26 \mathrm{E}+04$ & $1.11 \mathrm{E}+02$ & $1.27 \mathrm{E}+04$ & $1.27 \mathrm{E}+05$ & yes \\
\hline
\end{tabular}




\begin{tabular}{|c|c|c|c|c|c|c|c|}
\hline Nuclide & Half-Life & & $\begin{array}{c}\text { Class A } \\
(\mathrm{mCi})\end{array}$ & $\begin{array}{c}\text { Class B } \\
(\mathrm{mCi})\end{array}$ & $\begin{array}{c}\text { Total } A+B \\
(m C i)\end{array}$ & $\begin{array}{l}30 \text { year total } \\
(\mathrm{mCi})\end{array}$ & $\begin{array}{c}\text { Pre- } \\
\text { Screened? }\end{array}$ \\
\hline$I-129$ & $1.57 \mathrm{E}+07$ & $\mathrm{yr}$ & $3.31 \mathrm{E}-01$ & $8.06 \mathrm{E}-02$ & $4.11 \mathrm{E}-01$ & $4.11 \mathrm{E}+00$ & \\
\hline$I-131$ & $8.04 \mathrm{E}+00$ & $\mathrm{~d}$ & $1.62 E+05$ & $1.87 \mathrm{E}+04$ & $1.81 E+05$ & $1.81 E+06$ & yes \\
\hline IR-192 & $7.40 \mathrm{E}+01$ & d & $1.12 \mathrm{E}+01$ & & $1.12 \mathrm{E}+01$ & $1.12 E+02$ & yes \\
\hline MN-54 & $3.13 E+02$ & $\mathrm{~d}$ & $1.82 E+06$ & $1.58 \mathrm{E}+06$ & $3.40 \mathrm{E}+06$ & $3.40 \mathrm{E}+07$ & yes \\
\hline NA-22 & $2.60 \mathrm{E}+00$ & $y^{t}$ & $1.37 \mathrm{E}+01$ & $2.02 \mathrm{E}-02$ & $1.37 \mathrm{E}+01$ & 1.37E+02 & \\
\hline NB-94 & $2.03 E+04$ & yr & $7.74 \mathrm{E}+00$ & $1.90 \mathrm{E}+01$ & $2.68 E+01$ & $2.68 E+02$ & \\
\hline NB-95 & $3.51 \mathrm{E}+01$ & $\mathrm{~d}$ & $2.33 E+03$ & $1.48 \mathrm{E}+03$ & $3.81 E+03$ & $3.81 E+04$ & yes \\
\hline NI-59 & $7.50 \mathrm{E}+04$ & $\mathrm{yr}$ & $1.52 E+03$ & $3.52 \mathrm{E}+03$ & $5.03 E+03$ & $5.03 E+04$ & \\
\hline NI-63 & $1.00 \mathrm{E}+02$ & $\mathrm{yr}$ & $1.25 \mathrm{E}+05$ & $4.69 E+05$ & $5.94 E+05$ & $5.94 E+06$ & \\
\hline NP-237 & $2.10 \mathrm{E}+06$ & $\mathrm{yr}$ & $0.00 E+00$ & & $0.00 \mathrm{E}+00$ & $0.00 \mathrm{E}+00$ & \\
\hline P-32 & $1.43 E+01$ & $\mathrm{~d}$ & $1.87 \mathrm{E}+03$ & $1.25 \mathrm{E}+02$ & $2.00 \mathrm{E}+03$ & $2.00 \mathrm{E}+04$ & yes \\
\hline P-33 & $2.54 \mathrm{E}+01$ & $\mathrm{~d}$ & $1.20 E+01$ & $1.00 \mathrm{E}-01$ & $1.21 \mathrm{E}+01$ & $1.21 E+02$ & yes \\
\hline PB-210 & $2.23 E+01$ & $\mathrm{yr}$ & $7.33 E+00$ & $6.50 \mathrm{E}-02$ & $7.39 \mathrm{E}+00$ & $7.39 E+01$ & \\
\hline PM-147 & $2.62 \mathrm{E}+00$ & $\mathrm{yr}$ & $9.04 E+02$ & $1.73 E+00$ & $9.05 E+02$ & $9.05 E+03$ & \\
\hline PO-210 & $1.38 \mathrm{E}+02$ & $\mathrm{~d}$ & $1.06 E+01$ & & $1.06 \mathrm{E}+01$ & $1.06 \mathrm{E}+02$ & yes \\
\hline PU-238 & $8.78 \mathrm{E}+01$ & $\mathrm{yr}$ & $1.33 E+01$ & $1.82 \mathrm{E}+01$ & $3.15 \mathrm{E}+01$ & $3.15 \mathrm{E}+02$ & \\
\hline PU-239 & $2.41 \mathrm{E}+04$ & yr & $1.73 E+01$ & $5.51 \mathrm{E}+00$ & $2.28 \mathrm{E}+01$ & $2.28 \mathrm{E}+02$ & \\
\hline PU-240 & $6.57 \mathrm{E}+03$ & yr & $3.49 E+00$ & $1.29 E+00$ & $4.79 E+00$ & $4.79 E+01$ & \\
\hline PU-241 & $1.44 E+01$ & yr & $1.51 E+03$ & $1.12 E+03$ & $2.63 E+03$ & $2.63 E+04$ & \\
\hline PU-242 & $8.30 \mathrm{E}+07$ & yr & $0.00 \mathrm{E}+00$ & & $0.00 \mathrm{E}+00$ & $0.00 \mathrm{E}+00$ & \\
\hline RA-226 & $1.60 \mathrm{E}+03$ & $\mathrm{yr}$ & $7.43 \mathrm{E}-01$ & & $7.43 \mathrm{E}-01$ & $7.43 E+00$ & \\
\hline RB-86 & $1.02 \mathrm{E}+00$ & $\min$ & $1.25 \mathrm{E}+02$ & $1.00 \mathrm{E}-02$ & $1.25 E+02$ & $1.25 E+03$ & yes \\
\hline RU-103 & $3.94 \mathrm{E}+0 \mathrm{I}$ & $\mathrm{d}$ & $3.26 \mathrm{E}+01$ & $3.11 \mathrm{E}+02$ & $3.44 E+02$ & $3.44 E+03$ & yes \\
\hline RU-106 & $3.68 \mathrm{E}+02$ & d & $3.48 \mathrm{E}+02$ & & $3.48 E+02$ & $3.48 E+03$ & yes \\
\hline S-35 & $8.74 \mathrm{E}+01$ & d & $4.89 E+03$ & $1.43 \mathrm{E}+02$ & $5.03 E+03$ & $5.03 E+04$ & yes \\
\hline SB-124 & $6.02 E+01$ & d & $2.92 \mathrm{E}+03$ & $9.41 E+03$ & $1.23 \mathrm{E}+04$ & $1.23 \mathrm{E}+05$ & yes \\
\hline SB-125 & $2.77 \mathrm{E}+00$ & $\mathrm{yr}$ & $4.17 \mathrm{E}+03$ & $5.78 \mathrm{E}+03$ & $9.95 E+03$ & $9.95 E+04$ & \\
\hline $\mathrm{SC}-46$ & $8.38 E+01$ & $\mathrm{~d}$ & $2.16 \mathrm{E}+01$ & & $2.16 \mathrm{E}+01$ & $2.16 \mathrm{E}+02$ & yes \\
\hline SE-75 & $1.20 \mathrm{E}+02$ & $\mathrm{~d}$ & $5.42 \mathrm{E}-01$ & & $5.42 \mathrm{E}-01$ & $5.42 \mathrm{E}+00$ & yes \\
\hline SN-113 & $2.14 \mathrm{E}+01$ & $\min$ & $4.51 \mathrm{E}+01$ & $2.28 \mathrm{E}+03$ & $2.32 \mathrm{E}+03$ & $2.32 E+04$ & yes \\
\hline Sr-85 & $6.48 E+01$ & $d$ & $1.66 \mathrm{E}+01$ & & $1.66 \mathrm{E}+01$ & $1.66 \mathrm{E}+02$ & yes \\
\hline Sr-89 & $5.05 \mathrm{E}+01$ & $\mathrm{~d}$ & $3.56 \mathrm{E}+03$ & $2.78 E+04$ & $3.14 E+04$ & $3.14 \mathrm{E}+05$ & yes \\
\hline Sr-90 & $2.91 E+01$ & yt & $4.04 E+03$ & $1.32 \mathrm{E}+04$ & $1.73 E+04$ & $1.73 E+05$ & \\
\hline Ta-182 & $1.15 E+02$ & $\mathrm{~d}$ & $2.85 \mathrm{E}+01$ & $1.00 \mathrm{E}-03$ & $2.85 E+01$ & $2.85 E+02$ & yes \\
\hline Tc-99 & $2.13 E+05$ & $y T$ & $6.99 E+00$ & $3.94 E+00$ & $1.09 E+01$ & $1.09 E+02$ & \\
\hline $\mathrm{Te}-125 \mathrm{M}$ & $5.80 \mathrm{E}+01$ & $\mathrm{~d}$ & $2.02 E+01$ & $4.91 E+00$ & $2.51 E+01$ & $2.51 \mathrm{E}+02$ & yes \\
\hline Th (NAT.) & -- & --- & $2.39 E+01$ & & $2.39 E+01$ & $2.39 \mathrm{E}+02$ & yes \\
\hline TH-228 & $1.91 \mathrm{E}+00$ & $\mathrm{yr}$ & $7.37 \mathrm{E}-02$ & & 7.37E-02 & 7.37E-01 & \\
\hline Th-230 & $7.70 \mathrm{E}+04$ & $\mathrm{yr}$ & $3.98 \mathrm{E}-01$ & & $3.98 \mathrm{E}-01$ & $3.98 \mathrm{E}+00$ & \\
\hline Th-232 & $1.41 E+10$ & yr & $3.79 E+01$ & & $3.79 \mathrm{E}+01$ & $3.79 E+02$ & \\
\hline Th-234 & $2.4 \mathrm{IE}+01$ & $\mathrm{~d}$ & $1.63 E+01$ & & $1.63 E+01$ & $1.63 \mathrm{E}+02$ & yes \\
\hline TRU & -.- & --- & $3.97 \mathrm{E}+01$ & & $3.97 \mathrm{E}+01$ & $3.97 \mathrm{E}+02$ & yes \\
\hline U (NAT.) & -- & --- & $1.87 \mathrm{E}+01$ & & $1.87 \mathrm{E}+01$ & $1.87 \mathrm{E}+02$ & yes \\
\hline $\mathrm{U}-232$ & $7.20 \mathrm{E}+01$ & $\mathrm{yr}$ & $2.48 \mathrm{E}-01$ & & $2.48 \mathrm{E}-01$ & $2.48 \mathrm{E}+00$ & \\
\hline $\mathrm{U}-233$ & $1.59 E+05$ & $\mathrm{yr}$ & $1.92 \mathrm{E}+01$ & & $1.92 \mathrm{E}+01$ & $1.92 E+02$ & \\
\hline $\mathrm{U}-234$ & $2.45 E+05$ & $\mathrm{yr}$ & $8.28 \mathrm{E}+00$ & & $8.28 \mathrm{E}+00$ & $8.28 \mathrm{E}+01$ & \\
\hline
\end{tabular}




\begin{tabular}{lccccccc}
\hline Nuclide & Half-Life & & $\begin{array}{c}\text { Class A } \\
(\mathbf{m C i})\end{array}$ & $\begin{array}{c}\text { Class B } \\
(\mathbf{m C i})\end{array}$ & $\begin{array}{c}\text { Total A+B } \\
(\mathbf{m C i})\end{array}$ & $\begin{array}{c}\text { 30 year total } \\
(\mathbf{m C i})\end{array}$ & $\begin{array}{c}\text { Pre- } \\
\text { Screened? }\end{array}$ \\
\hline $\mathrm{U}-235$ & $7.04 \mathrm{E}+08$ & $\mathrm{yr}$ & $2.81 \mathrm{E}-01$ & & $2.81 \mathrm{E}-01$ & $2.81 \mathrm{E}+00$ & \\
$\mathrm{U}-236$ & $2.43 \mathrm{E}+07$ & $\mathrm{yr}$ & $0.00 \mathrm{E}+00$ & & $0.00 \mathrm{E}+00$ & $0.00 \mathrm{E}+00$ & \\
$\mathrm{U}-238$ & $4.47 \mathrm{E}+09$ & $\mathrm{yr}$ & $2.52 \mathrm{E}+01$ & & $2.52 \mathrm{E}+01$ & $2.52 \mathrm{E}+02$ & \\
$\mathrm{Y}-88$ & $1.07 \mathrm{E}+02$ & $\mathrm{~d}$ & $5.45 \mathrm{E}-01$ & & $5.45 \mathrm{E}-01$ & $5.45 \mathrm{E}+00$ & yes \\
$\mathrm{Zn}-65$ & $2.44 \mathrm{E}+02$ & $\mathrm{~d}$ & $1.79 \mathrm{E}+06$ & $2.59 \mathrm{E}+06$ & $4.39 \mathrm{E}+06$ & $4.39 \mathrm{E}+07$ & yes \\
Zr-95 & $6.40 \mathrm{E}+01$ & $\mathrm{~d}$ & $6.14 \mathrm{E}+02$ & $3.06 \mathrm{E}+02$ & $9.20 \mathrm{E}+02$ & $9.20 \mathrm{E}+03$ & yes \\
\hline
\end{tabular}

Table A-2 Best estimate inventories for Class $C$ waste. The 30 year total is 10 times the "Total Class C" column and was used in the groundwater analysis.

\begin{tabular}{|c|c|c|c|c|c|}
\hline Nuclide & Half-Life & & $\begin{array}{c}\text { Class C } \\
(\mathrm{mCi})\end{array}$ & $\begin{array}{c}\text { Total Class C } \\
(\mathrm{mCi})\end{array}$ & $\begin{array}{c}\text { Pre- } \\
\text { Screened? }\end{array}$ \\
\hline $\mathrm{Ag}-110 \mathrm{M}$ & $2.50 \mathrm{E}+02$ & $d$ & $1.42 E+03$ & $1.42 \mathrm{E}+04$ & yes \\
\hline $\mathrm{Am}-241$ & $4.32 E+02$ & yr & $1.51 \mathrm{E}+00$ & $1.51 E+01$ & \\
\hline Am-243 & $7.38 \mathrm{E}+03$ & yr & $1.44 \mathrm{E}-03$ & $1.44 \mathrm{E}-02$ & \\
\hline $\mathrm{Ba}-133$ & $1.05 \mathrm{E}+01$ & $\mathrm{yr}$ & $1.00 \mathrm{E}-02$ & $1.00 \mathrm{E}-01$ & \\
\hline $\mathrm{Ba}-140$ & $1.28 \mathrm{E}+01$ & $\mathrm{~d}$ & $1.73 E+01$ & $1.73 E+02$ & yes \\
\hline $\mathrm{Be}-7$ & $5.34 \mathrm{E}+01$ & d & $1.22 \mathrm{E}+03$ & $1.22 \mathrm{E}+04$ & yes \\
\hline C-14 & $5.73 E+03$ & yr & $8.84 \mathrm{E}+03$ & $8.84 E+04$ & \\
\hline CD-109 & $1.27 \mathrm{E}+00$ & $\mathrm{yr}$ & $7.63 \mathrm{E}-02$ & 7.63E-01 & \\
\hline CE-139 & $1.38 \mathrm{E}+02$ & d & 2.30E-03 & $2.30 \mathrm{E}-02$ & yes \\
\hline Ce-141 & $3.25 \mathrm{E}+01$ & d & $6.99 \mathrm{E}-01$ & $6.99 E+00$ & yes \\
\hline Ce-144 & $2.84 \mathrm{E}+02$ & d & $3.86 E+04$ & $3.86 \mathrm{E}+05$ & yes \\
\hline CM-242 & $1.63 E+02$ & d & $3.46 \mathrm{E}+01$ & $3.46 E+02$ & yes \\
\hline CM-243 & $2.85 E+01$ & $\mathrm{yr}$ & $5.74 \mathrm{E}-01$ & $5.74 \mathrm{E}+00$ & \\
\hline CM-244 & $1.81 \mathrm{E}+01$ & yr & $1.86 \mathrm{E}+00$ & $1.86 \mathrm{E}+01$ & \\
\hline Co-57 & $2.71 \mathrm{E}+02$ & $\mathrm{~d}$ & $3.03 E+03$ & $3.03 E+04$ & yes \\
\hline Co- 58 & $7.08 \mathrm{E}+01$ & $\mathrm{~d}$ & $9.19 E+05$ & $9.19 E+06$ & yes \\
\hline Co-60 & $5.27 \mathrm{E}+00$ & $\mathrm{yr}$ & $7.75 \mathrm{E}+07$ & $7.75 \mathrm{E}+08$ & \\
\hline $\mathrm{Cr}-51$ & $2.77 \mathrm{E}+01$ & $\mathrm{~d}$ & $1.23 E+06$ & $1.23 E+07$ & yes \\
\hline Cs- 134 & $2.06 \mathrm{E}+00$ & $y \tau$ & $5.73 E+03$ & $5.73 E+04$ & \\
\hline Cs- 137 & $3.00 \mathrm{E}+01$ & $y r$ & $4.22 E+04$ & $4.22 E+05$ & \\
\hline Eu-154 & $8.60 \mathrm{E}+00$ & yr & $0.00 E+00$ & $0.00 \mathrm{E}+00$ & \\
\hline Eu-155 & $4.96 E+00$ & $y r$ & $0.00 \mathrm{E}+00$ & $0.00 \mathrm{E}+00$ & \\
\hline $\mathrm{Fe}-55$ & $2.70 E+00$ & $y^{\prime} r$ & $1.03 E+08$ & $1.03 E+09$ & \\
\hline Fe-59 & $4.46 E+01$ & $\mathrm{~d}$ & $8.15 E+04$ & $8.15 E+05$ & yes \\
\hline $\mathrm{H}-3$ & $1.23 \mathrm{E}+01$ & yr & $3.77 E+05$ & $3.77 E+06$ & \\
\hline I- 129 & $1.57 \mathrm{E}+07$ & yr & $8.65 \mathrm{E}-02$ & $8.65 \mathrm{E}-01$ & \\
\hline$[-131$ & $8.04 E+00$ & $\mathrm{~d}$ & $9.48 \mathrm{E}+02$ & $9.48 E+03$ & yes \\
\hline $\mathrm{Mn}-54$ & $3.13 E+02$ & d & $1.08 E+07$ & $1.08 E+08$ & yes \\
\hline Nb-94 & $2.03 E+04$ & $\mathrm{yr}$ & $1.58 \mathrm{E}+02$ & $1.58 \mathrm{E}+03$ & \\
\hline $\mathrm{Nb}-95$ & $3.51 \mathrm{E}+01$ & $\mathrm{~d}$ & $3.06 \mathrm{E}+04$ & $3.06 E+05$ & yes \\
\hline $\mathrm{Ni}-59$ & $7.50 \mathrm{E}+04$ & yr & $5.90 \mathrm{E}+04$ & $5.90 E+05$ & \\
\hline $\mathrm{Ni}-63$ & $1.00 \mathrm{E}+02$ & $\mathrm{yr}$ & $4.38 \mathrm{E}+06$ & $4.38 \mathrm{E}+07$ & \\
\hline $\mathrm{Np}-237$ & $2.10 \mathrm{E}+06$ & $\mathrm{yr}$ & $7.86 \mathrm{E}-03$ & $7.86 \mathrm{E}-02$ & \\
\hline
\end{tabular}




\begin{tabular}{|c|c|c|c|c|c|}
\hline Nuclide & Half-Life & & $\begin{array}{c}\text { Class C } \\
(\mathrm{mCi}) \\
\end{array}$ & $\begin{array}{c}\text { Total Class C } \\
(\mathrm{mCi})\end{array}$ & $\begin{array}{c}\text { Pre- } \\
\text { Screened? }\end{array}$ \\
\hline P-32 & $1.43 \mathrm{E}+0 \mathrm{l}$ & $\mathrm{d}$ & $1.59 E+03$ & $1.59 \mathrm{E}+04$ & yes \\
\hline $\mathrm{Pb}-10$ & $2.23 E+01$ & $\mathrm{yr}$ & $2.00 \mathrm{E}+01$ & $2.00 \mathrm{E}+02$ & \\
\hline$P m-47$ & $2.62 \mathrm{E}+00$ & $\mathrm{yr}$ & $0.00 \mathrm{E}+00$ & $0.00 E+00$ & \\
\hline $\mathrm{Pu}-238$ & $8.78 E+01$ & yr & $1.22 E+01$ & $1.22 E+02$ & \\
\hline Pu-239 & $2.41 \mathrm{E}+04$ & $\mathrm{yr}$ & $9.71 \mathrm{E}-01$ & $9.71 E+00$ & \\
\hline $\mathrm{Pu}-240$ & $6.57 E+03$ & yr & $0.00 \mathrm{E}+00$ & $0.00 \mathrm{E}+00$ & \\
\hline $\mathrm{Pu}-241$ & $1.44 E+01$ & yr & $1.86 E+03$ & $1.86 E+04$ & \\
\hline Pu-242 & $8.30 E+07$ & $\mathrm{yr}$ & 7.93E-06 & 7.93E-05 & \\
\hline $\mathrm{Ra}-226$ & $1.60 \mathrm{E}+03$ & $\mathrm{yr}$ & $0.00 \mathrm{E}+00$ & $0.00 \mathrm{E}+00$ & \\
\hline $\mathrm{Ru}-103$ & $3.94 \mathrm{E}+01$ & d & $2.08 E+03$ & $2.08 E+04$ & yes \\
\hline Ru-106 & $3.68 E+02$ & $d$ & $3.03 E+03$ & $3.03 E+04$ & yes \\
\hline Sb-124 & $6.02 E+01$ & $d$ & $2.54 \mathrm{E}+02$ & $2.54 \mathrm{E}+03$ & yes \\
\hline Sb- 125 & $2.77 \mathrm{E}+00$ & $\mathrm{yr}$ & $3.55 E+04$ & $3.55 E+05$ & \\
\hline $\mathrm{Sn}-113$ & $2.14 E+01$ & $\min$ & $2.74 \mathrm{E}+02$ & $2.74 \mathrm{E}+03$ & yes \\
\hline Sr-89 & $5.05 E+01$ & $\mathrm{~d}$ & $6.09 E+02$ & $6.09 \mathrm{E}+03$ & yes \\
\hline Sr-90 & $2.91 E+01$ & yr & $1.30 \mathrm{E}+02$ & $1.30 E+03$ & \\
\hline Tc-99 & $2.13 E+05$ & $\mathrm{yr}$ & $5.61 \mathrm{E}-01$ & $5.61 \mathrm{E}+00$ & \\
\hline $\mathrm{Te}-125 \mathrm{M}$ & $5.80 \mathrm{E}+01$ & d & $9.02 \mathrm{E}+01$ & $9.02 E+02$ & yes \\
\hline TRU & --- & --- & $4.67 \mathrm{E}-01$ & $4.67 \mathrm{E}+00$ & yes \\
\hline U-234 & $2.45 E+05$ & yr & $0.00 \mathrm{E}+00$ & $0.00 \mathrm{E}+00$ & \\
\hline $\mathrm{U}-235$ & $7.04 \mathrm{E}+08$ & $\mathrm{yr}$ & $0.00 \mathrm{E}+00$ & $0.00 \mathrm{E}+00$ & \\
\hline U-236 & $2.43 E+07$ & yr & $0.00 \mathrm{E}+00$ & $0.00 \mathrm{E}+00$ & \\
\hline U-238 & $4.47 E+09$ & $\mathrm{yr}$ & $0.00 \mathrm{E}+00$ & $0.00 \mathrm{E}+00$ & \\
\hline Y-88 & $1.07 \mathrm{E}+02$ & d & $7.60 \mathrm{E}-03$ & $7.60 \mathrm{E}-02$ & yes \\
\hline $\mathrm{Zn}-65$ & $2.44 E+02$ & $d$ & $3.93 E+05$ & $3.93 \mathrm{E}+06$ & yes \\
\hline Zr-95 & $6.40 \mathrm{E}+0 \mathrm{l}$ & $\mathrm{d}$ & $4.04 E+03$ & $4.04 E+04$ & yes \\
\hline
\end{tabular}




\section{Appendix B \\ Concrete Degradation and Steel Reinforcement Corrosion}

A waste containment system consisting of a concrete outer shell. concrete overpacks. and sand backfill has been developed for use in the disposal of low-level radioactive waste (LLW) in Pennsylvania. The concrete overpacks provide primary containment during vault operations, and the concrete shell provides structural stability for the disposal and will also act as a hydrologic barrier until failure due to fracturing or complete degradation. The models described below provide estimates of concrete degradation and rebar corrosion due to reactions with soil water chemical constituents in contact with the concrete vault. A water composition data set was selected based on availability because a site has not been selected for the facility. The degradation processes have been modeled separately rather than integrated because degradation is typically dominated by a single process. Parametric estimates of degradation are ured to evaluate wide ranges of scenarios. Fractures that run the entire height of the vaults are assumed to occur at 500 years, and complete hydrologic failure occurs at 1.000 years as a first approximation. This assumption was based on calculations made for the Savannah River Plant Intermediate Level Non-Tritium " a〉te vaults. Because the vault design has not been finalized. the effort for this level of calculation is not warranted.

\section{Disposal Vault Description}

As discussed previously in the document, the vaults that will contain the Class $A$ and $B$ waste have dimensions of $122 \mathrm{~m} \times 610 \mathrm{~m} \times 7.6 \mathrm{~m}$ and Class $\mathrm{C}$ vaults have dimensions of $2+\mathrm{m} \times 49 \mathrm{~m} \times 3 \mathrm{~m}$. Vault floors and roofs will be comprised of 24-inch thick steel reinforced concrete. and the udewalls will be 20inch thick steel reinforced concrete with a minimum rebar coverage of 3 incher. The mix design used in the concrete degradation estimates is shown in Table 1.

Table 1. Concrete mix design for low-level waste vaults.

\begin{tabular}{|c|c|c|}
\hline Component & Weight Percent & Lbs/Yd \\
\hline \hline Type II OPC & 14.7 & 674 \\
\hline Fly Ash & 3.67 & 168.3 \\
\hline Sand & 29.88 & 1.370 \\
\hline Fine Aggregate & 5.75 & 26.3 .6 \\
\hline Small Aggregate & 14.7 & 674 \\
\hline Medium Aggregate & 31.25 & 14.32 .8 \\
\hline Water & NA & 2.56 \\
\hline Total & & 4841 \\
\hline
\end{tabular}

Appendix B Page 1 


\section{Groundwater Chemistry Data}

A groundwater composition was selected from the literature based on availability because no site has been selected for the LLW facility at the time of these calculations. The groundwater composition selected (Table 2) is from a Mississippian Sandstone from Clawford County. PA (Todd 1980). How representative this water composition is of waters that will be present at the selected site is unknown. Degradation estimates have been made with varying component concentrations to evaluate the impact of different water compositions. The key component concentrations for concrete degradation are calcium. inorganic carbon, magnesium, sulfate. chloride. and oxygen. Refinements to the calculations based on site-specific water composition data may be needed upon final site selection.

Table 2. Groundwater composition data.

\begin{tabular}{|c|c|c|}
\hline Component & $\begin{array}{c}\text { Concentration } \\
(\mathrm{mg} / \mathrm{L})\end{array}$ & $\begin{array}{c}\text { Concentration } \\
(\mathrm{M})\end{array}$ \\
\hline \hline $\mathrm{SiO}_{2}$ & 14 & $2.33 \mathrm{E}-4$ \\
\hline $\mathrm{Fe}$ & 1.3 & $2.32 \mathrm{E}-5$ \\
\hline $\mathrm{Ca}$ & 44 & $1.10 \mathrm{E}-3$ \\
\hline $\mathrm{Mg}$ & 11 & $4.53 \mathrm{E}-4$ \\
\hline $\mathrm{Na}$ & 60 & $2.61 \mathrm{E}-3$ \\
\hline $\mathrm{K}$ & 4.1 & $1.05 \mathrm{E}-4$ \\
\hline $\mathrm{HCO}$ & 327 & $5.36 \mathrm{E}-3$ \\
\hline $\mathrm{SO}_{4}$ & 22 & $2.30 \mathrm{E}-4$ \\
\hline $\mathrm{Cl}_{3}$ & 4.4 & $1.24 \mathrm{E}-4$ \\
\hline $\mathrm{F}$ & 0.2 & $1.05 \mathrm{E}-5$ \\
\hline $\mathrm{NO}_{3}$ & 2.0 & $3.22 \mathrm{E}-5$ \\
\hline
\end{tabular}

Other parameters: Speculic Conductance 533. pH 7.4. PO 4.

Al. In. Cu. Zn. and CO.3 were below detection limsts.

\section{Concrete Degradation Modeling Approach}

The models described by Walton et al. (1990) were used to estimate concrete degradation and rebar corrosion processes. Concrete degradation processes considered are (1) magnesium and sulfate attack, (2) calcium leaching (both geologically and concrete controlled), and (3) carbonation. Rebar corrosion due to chloride iutack and oxygen limited corrosion are also considered for the vaults. A brief discussion of each process considered is provided along with estimated degradation and corrosion rates for the concrete and rebar. respectively. 


\section{Magnesium and Sulfate Attack}

Degradation of cement can involve reaction of magnesium $(\mathrm{Mg})$ and sulfate $\left(\mathrm{SO}_{4}\right)$ contained in contact water with a solid phase in the cement. The solid formed by the reaction has a greater volume than the solid it replaced (e.g., sulfate reacting with portlandite to form gypsum, monosulphoaluminate, and ettringite) causing stress. and possible cracking and/or exfoliation of the concrete. This type of concrete degradation has been modeled based on observations made during the Norwick Park study in which the loss of cement at concrete block corners was measured after 5 vears of exposure to a 0.19 mole/ $\mathrm{L} \mathrm{Nil}_{2} \mathrm{SO}_{4}$ solution. The loss rate indicates a linear relationship with time and is assumed to be proportional to $\mathrm{SO}_{4}$ concentration in the solution and tricalciumaluminate $(\mathrm{C} 3 \mathrm{~A})$ content in the cement. Concrete degradation in a $\mathrm{MgSO}_{4}$ solution is approximately twice that found in comparable $\mathrm{Na}_{2} \mathrm{SO}_{4}$ solutions. This is attributed to reactions between magnesium ( $\mathrm{Mg}$ ) and $\mathrm{C} 3 \mathrm{~A}$ as well as sulfate and $\mathrm{C} 3 \mathrm{~A}$ reactions. Therefore, the depth of degradation is estimated based on $\mathrm{Mg}$ and $\mathrm{SO}_{\downarrow}$ concentrations in the contact water and the amount of $\mathrm{C} 3 \mathrm{~A}$ in the cement (Equation 1).

$$
M S_{1}=0.55 \times C 3 \mathrm{~A} \times \mathrm{C}_{. M g S O_{4}} \times t
$$

where: $\quad \mathrm{MS}_{\mathrm{x}} \quad$ penetration depth of $\mathrm{Mg}$ plus $\mathrm{SO}_{+}$attack $(\mathrm{cm})$.

$$
\begin{array}{ll}
\mathrm{C} 3 \mathrm{~A} & \text { weight percent of } \mathrm{C} 3 \mathrm{~A} \text { in unhydrated cement (unitless) } \\
\mathrm{C}_{\mathrm{mgsot}} & \text { concentration of } \mathrm{Mg} \text { plus } \mathrm{SO}_{4} \text { in contact water (mole/L) } \\
\mathrm{t} & \text { time (years). }
\end{array}
$$

Initial estimates of $\mathrm{MgSO}_{4}$ degradation used a C.3A value of 8 weight percent (Rust Geotech) and a $\mathrm{Mg}$ plus $\mathrm{SO}_{4}$ concentration of $6.83 \mathrm{E}-4$ mole/Liter (see groundwater composition section), respectively. The $\mathrm{C} 3 \mathrm{~A}$ value is an upper bound as specified in the concrete mix design. These parameter values result in approximately $15-\mathrm{cm}$ (6 inches) of concrete degradation and does not impact the hydrologic integrity of the vault until well after the failure time of 1,000 years used in the transport modeling.

A parametric evaluation of magnesium and sulfate attack was conducted in order to address some of the uncertainties in the model and the parameter values used in the calculation. Concrete degradation as a function of $\mathrm{MgSO}_{+}$concentration in the contact water and time is shown in Figure 2. These parameters were selected because all other parameters are constants in this case. The estimate 1 an conducted for the time to complete hydrologic failure ( 1.000 years) used in the transport modeling. Contact water Mg plus $\mathrm{SO}_{4}$ concentrations were varied an order of magnitude above and below the concentration indicated in the water chemistry data $\left(\sim 10^{-3} \mathrm{M}\right)$. 


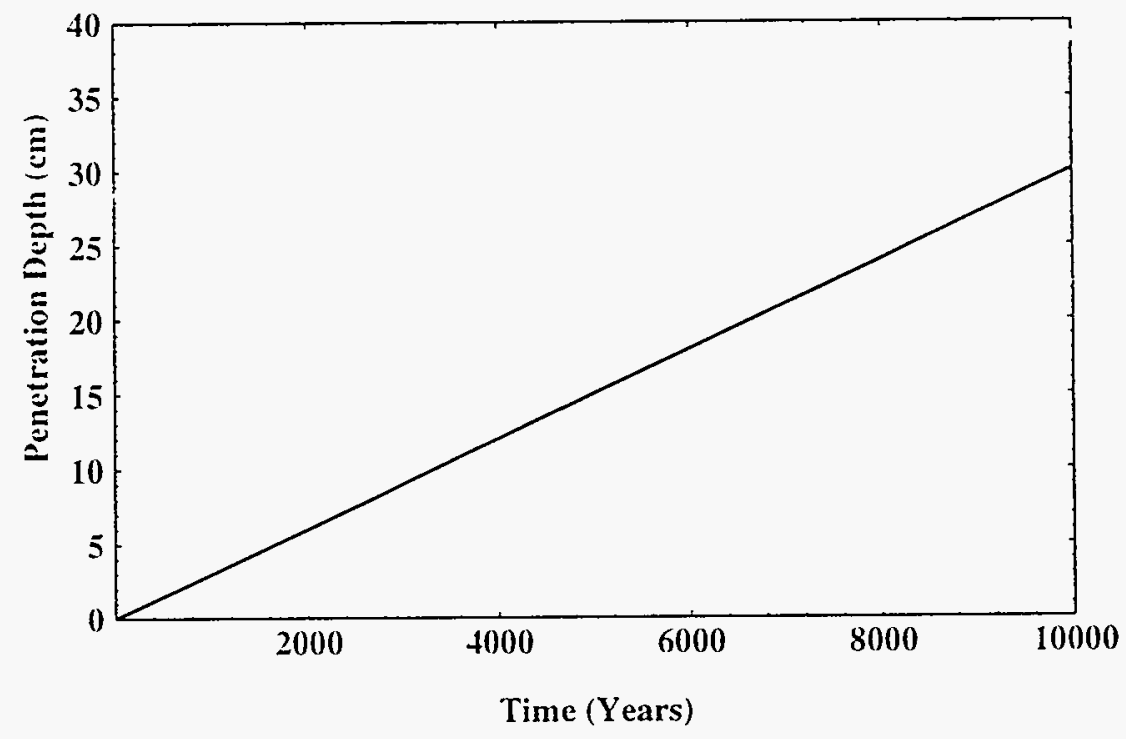

Figure 1. Magnesium and sulfate attack on concrete using 8 weight percent tricalcium aluminate and a $\mathrm{Mg}$ plus $\mathrm{SO} 4$ concentration of $6.83 \mathrm{E}-4 \mathrm{M}$.

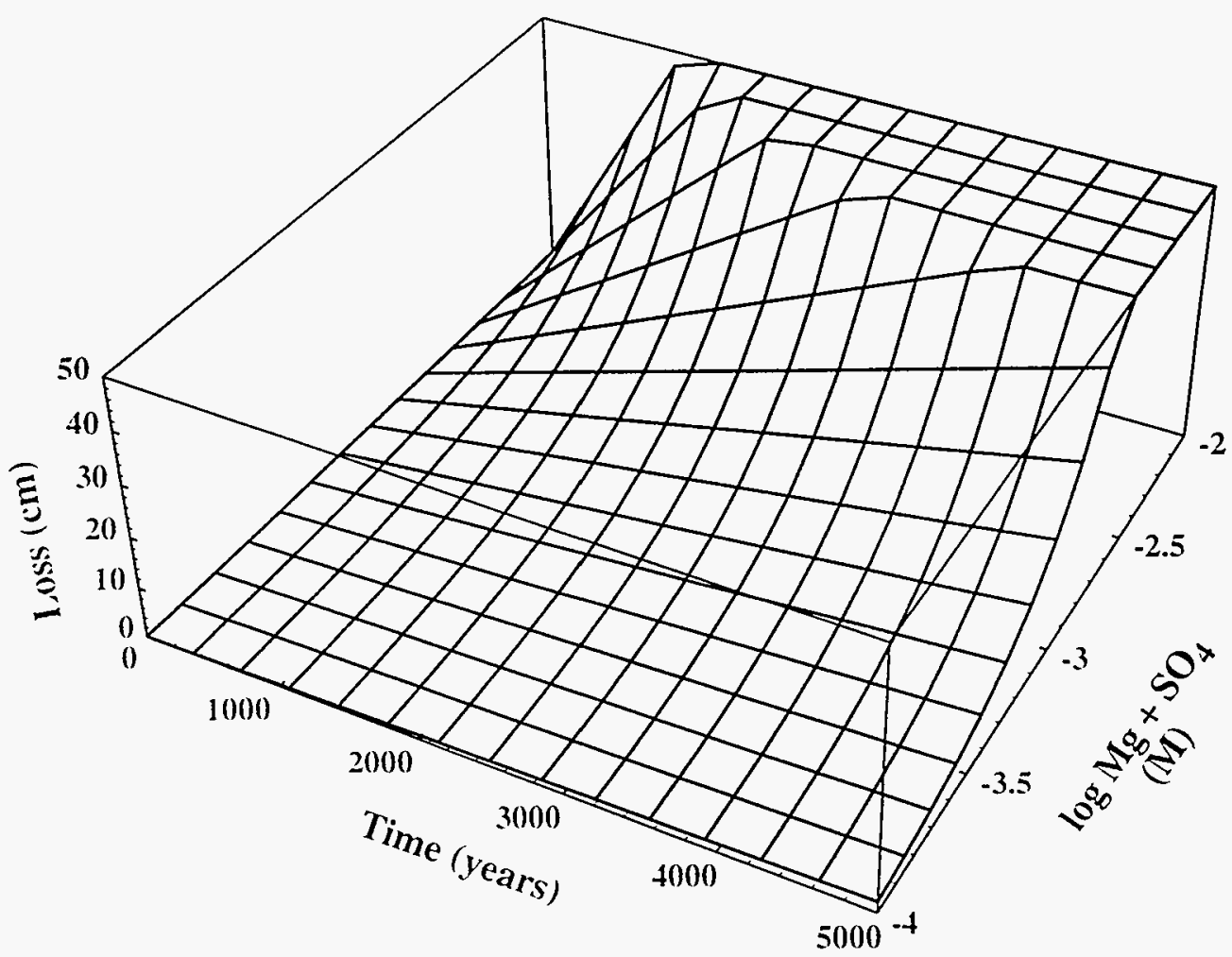

Figure 2. Concrete degradation as a function of time and magnesium plus sulfate water concentrations. 
The parametric evaluation indicates that over the time period of interest. magnesium plus sulfate concentrations less than $10^{-3} \mathrm{M}$ have negligible impact on the concrete vaults from a hydrologic performance perspective (20-inch or 50-cm thickness). $\mathrm{Mg}$ plus $\mathrm{SO}_{+}$concentrations between $10^{-3}$ to $10^{-2}$ $\mathrm{M}$ predicted degradation ranges from approximately 30 to 100 percent degradation at 5,000 years. At the maximum concentration used, the vault will be severely degraded at 1,000 years. Without site-specific information, it is not possible to determine the potential for these high concentrations of $\mathrm{Mg}_{\mathrm{g}}$ plus $\mathrm{SO}_{4}$. In general, $\mathrm{Mg}$ and $\mathrm{SO}_{4}$ concentrations in the upper range selected in this evaluation are present in arid environments and are not likely in the northeastem L.S. Sulfur resistant cements. which contain less C3A, could be used to lessen the impact of high concentration $\mathrm{Mg}$ plus SO4 solutior , if the se are found to be a problem after a site has been selected. It has also been found that reducing the 1 ater-to-cement ratio will improve the lifetime of concrete significantly due to the associated decrease in cement porosity and permeability (Stark, 1982).

\section{Calcium Leaching}

Leaching of cement components occurs due to water contact with the cements under conditions of high water percolation. The movement of water through the cement causes the initial loss of alkali components (e.g., Na and K) with later removal of calcium hydroxide, which results in reduced cement strength. Experimental and modeling techniques (Atkinson. 1985; Atkinson et al., 1988) of the leaching process have resulted in the identification of four stages in the leaching process:

1) Alkali metal oxides and hydroxides control $\mathrm{pH}$ at approximately 13 .

2) Controls $\mathrm{pH}$ at 12.5 following leaching of alkali metals.

3) Calcium silicate hydrate gel phases begin to dissolve incongruently following $\left(0 \ldots\right.$ of $\mathrm{Ca}(\mathrm{OH})_{2}$ and the pH slowly moves to 10.5 .

4) Congruent dissolution of calcium silicate hydrate gel holds $\mathrm{pH}$ at 10.5 .

Two models have been developed to describe leaching based on the material controlling the rate of degradation (i.e., concrete and geology controlled leaching). Each of these models have some common parameters, although the geology controlled leaching model requires additional parameters that involve the properties of the surrounding material. Concrete controlled leaching has been described by a shrinking core model (Atkinson and Hearne. 1984). This model assumes that removal of Ca from the exterior of the concrete is rapid relative to the movement of Ca through the concrete (i.e.. diffuvon controlled release). This results in the formation of a sharply delineated rind of altered concrete surrounding an intact inner zone of concrete.

Concrete controlled leaching assumes that water tends to flow through the more permeable materials that surround the intact concrete structure rather than into the concrete. The permeability and subsequent flow rate in leached/degraded concrete will be greater than for intact concrete. This mat ledd to conditions such that diffusion is no longer the dominant mode of transport. The intrinsic diffusion coefficient $\left(D_{1}\right)$ in the model is for the degraded portion of the concrete (Equation 2). An initial estimate of $10^{-6} \mathrm{~cm}^{2} / \mathrm{s}$ for the diffusion coefficient was used for modeling. This is a conservative value considermg that the free water diffusion coefficient is $10^{-5} \mathrm{~cm}^{2} / \mathrm{s}$. Calcium concentrations in the pore water. groundwater. and bulk concrete are 2.7E-3 moles/Liter (Walton et al.. 1990). $1.1 \mathrm{E}-3$ moles/Liter ( $44 \mathrm{ppm}$ ). and $1.85 \mathrm{moles} / \mathrm{L}$, respectively. 


$$
C L_{x}=\left(2 D i\left(\frac{C a_{p u}-C a_{g w}}{C a_{c s}}\right) t\right)^{1 / 2}
$$

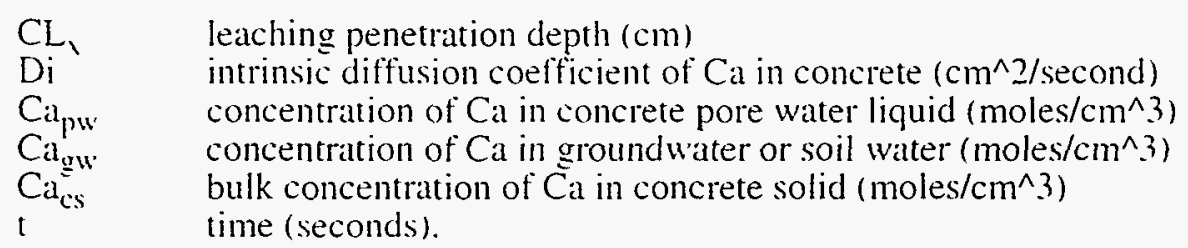

Estimated degradation resulting from concrete controlled leaching with the parameter values listed is negligible. At 5.000 years. the depth of degradation is approximately half a centimeter (0.2 inches). Increasing the diffusion coefficient to its maximum value of $10^{-5} \mathrm{~cm}^{2} / \mathrm{s}$ results in only $1.5-\mathrm{cm}$ of degradation (see Appendix B Attachmentl for degradation plots). The diffusion coefficient was varied to this extent because this is the parameter that is the most poorly defined. Using the free water diffusion coefficient insures that the evaluation is conservative from this perspective.

Geologically Controlled Calcium Leaching becomes important when diffusion of calcium into the material surrounding the concrete controls the leaching rate. Atkinson and Hearne (1984) developed a model to evaluate leaching under these conditions (Equation 3). In this case, the parameters related to transport are for the geologic material and not the concrete.

The lack of site information requires the use of hypothetical parameter values for the media properties that surround the concrete vaults. The porosity of the geologic material is assumed to be 30 percent, the retardation factor for calcium in the geologic material is 5 , and the effective diffusion coefficient is assumed to be $10^{-6} \mathrm{~cm}^{2} / \mathrm{s}$.

$$
G L x=2 \phi \frac{C a_{p w}-C a_{g w}}{C a_{c \cdot s}}\left(\frac{R_{d} D_{e} t}{\pi}\right)^{1 / 2}
$$

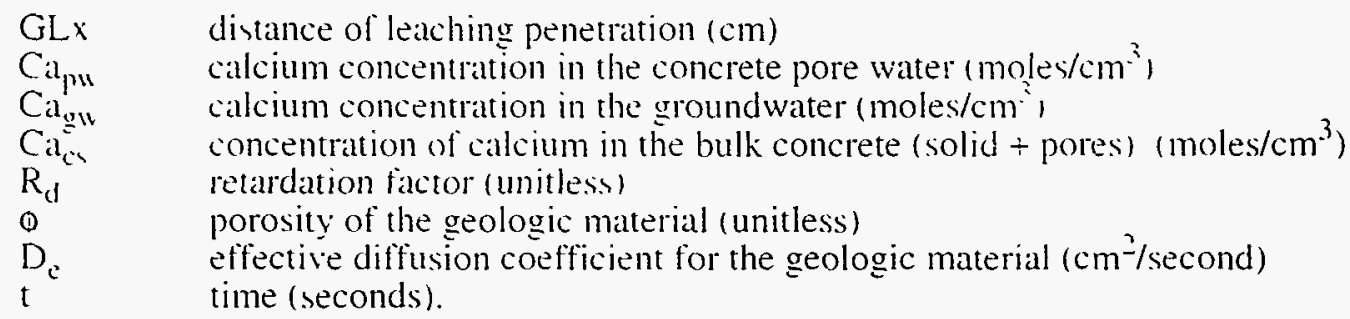

As with concrete controlled leaching, no significant loss of concrete is predicted considering geologically controlled leaching. The leaching evaluation may need to be updated after a site has been selected and characterized for the low-level radioactive waste disposal facility to determine if the assumed values are approprate. 


\section{Carbonation}

Carbonation involves the reaction of carbonate in infiltrating water or carbon dioxide in the vapor phase with calcium hydroxide in cement to form carbonates. This process does not decrease the effective thickness of the concrete as other degradation processes, but it can cause minor shrinkage or decrease the $\mathrm{pH}$ of the cement. Shrinkage due to carbonation is thought to occur as minerals are removed from the areas of compressive stress and redeposited in regions of lower stress. The strength of concrete generally increases under carbonation with the exception of high sulfate concretes. Carbonation forces the local $\mathrm{pH}$ toward neutrality, going from over 12 to about 8 . thereby removing some chemical barrier benefits of the concrete and providing a potentially corrosive environment for steel reinforcements if water. moisture and oxygen can penetrate.

The rate of carbonation is dependent upon water saturation or relative humidity of the environment. As relative humidity increases from 0 to $100 \%$, the rate of carbonation passes through a maximum at $50 \%$ (Verbeck, 1958). The maximum rate of carbonation occurs because the availability of water limits the reaction of carbon dioxide with calcium hydroxide to form calcium carbonate up to a relative humidity of $50 \%$. The decrease in carbonation above $50 \%$ relative humidity results from the decrease in the diffusion rate of carbon dioxide as the water content increases in the concrete (vapor phase diffusion is approximately four orders of magnitude more rapid than aqueous diffusion). Aqueous phase diffusion generally controls the transport of carbon dioxide in concrete even in the vadose zone because the concrete tends to stay saturated due to small pore sizes relative to surrounding material.

A shrinking core model for carbonation can be formulated by evaluating the migration rate of carbon dioxide into the saturated concrete in relation to the initial amount of calcium hydroxide (Equation 4). The assumption that $40 \%$ of the total $\mathrm{CaO}$ is hydrated $\left(\mathrm{Ca}(\mathrm{OH})_{2}\right)$ has been used in the calculations. Complete hydration of the cement seldom occurs and a value of $40 \%$ is a reasonable approximation of the probable hydration level. The range of $\mathrm{CaO}$ for portland cement ranges from 60 to $67 \%$ with a value of $65 \%$ being selected for the carbonation estimates. The range of diffusion coefficient for carbonate used in the calculations was from $10^{-8}$ to $10^{-5} \mathrm{~cm}^{2} / \mathrm{sec}$. These values bound the diffusion of carbonate in intact concrete to the free water diffusion coefficient. respectively. The bicarbonate concentration in the Pennsylvania groundwater composition used in the calculations is $5.36 \mathrm{E}-3 \mathrm{M}(.327 \mathrm{ppm})$.

$$
\left.C a r b_{1}=2 D, \frac{C_{g u}}{C_{a b}}\right)^{1} 2
$$

$\begin{array}{ll}\text { Carb } & \text { distance of carbonation penetration }(\mathrm{cm}) \\ \mathrm{D}_{\mathrm{i}} & \text { intrinsic diffusion coefficient of } \mathrm{Ca} \text { in concrete }\left(\mathrm{cm}^{2} / \mathrm{second}\right) \\ \mathrm{C}_{\mathrm{gw}} & \left.\text { concentration of total inorganic carbon in ground or soil water (moles } / \mathrm{cm}^{3}\right) \\ \mathrm{Cag}_{\mathrm{b}} & \text { bulk concentration of calcium hydroxide in concrete solid (moles/cm } \\ \mathrm{t} & \text { time (seconds). }\end{array}$

Carbonation evaluatıons were made over a range of inorganic carbon concentrations of $5 \times 10^{-2}$ to $5 \times 10^{-4}$ $M$ to estimate the impact of variation in this parameter on the carbonation penetration over time. Results using a diffusion coefficient of $10^{-6} \mathrm{~cm}^{2} / \mathrm{s}$ indicate that the lower concentration will not penetrate the vault wall thickness until well after the 5,000 year time period. Also, the depth of carbonation will not reach the

\footnotetext{
Appendix B Page 7
} 
steel reinforcement (assuming a 3-inch coverage) before 4.000 years after the vaults are capped. High carbon concentrations show a much more rapid carbonation of the vault. In this case, the entire wall thickness will be carbonated in 1.400 years. Using the groundwater concentration for carbon estimates a $30-\mathrm{cm}$ carbonation depth at 5.000 years and approximately 300 years to reach the steel reinforcement. It is important to keep in mind that the diffusion coefficient used in these calculations is conservative for intact concrete and the that carbon concentrations may be higher than the groundwater at the actual site location.

The depth of carbonation has also been evaluated over a range of carbon diffusion coefficients ranging from intact concrete to diffusion in water (Figure 3). Results indicate that for diffusion coefficients pertaining to intact to partially intact concrete carbonation will not penetrate the entire vault wall thickness until after 5.000 years. The steel reinforcement may be subjected to reduced $\mathrm{pH}$ due to carbonation after 1,000 years for all but the completely intact concrete case. It may be beneficial to place the rebar deeper into the concrete to provide longer protection to the reinforcing steel if this does not pose a construction problem.

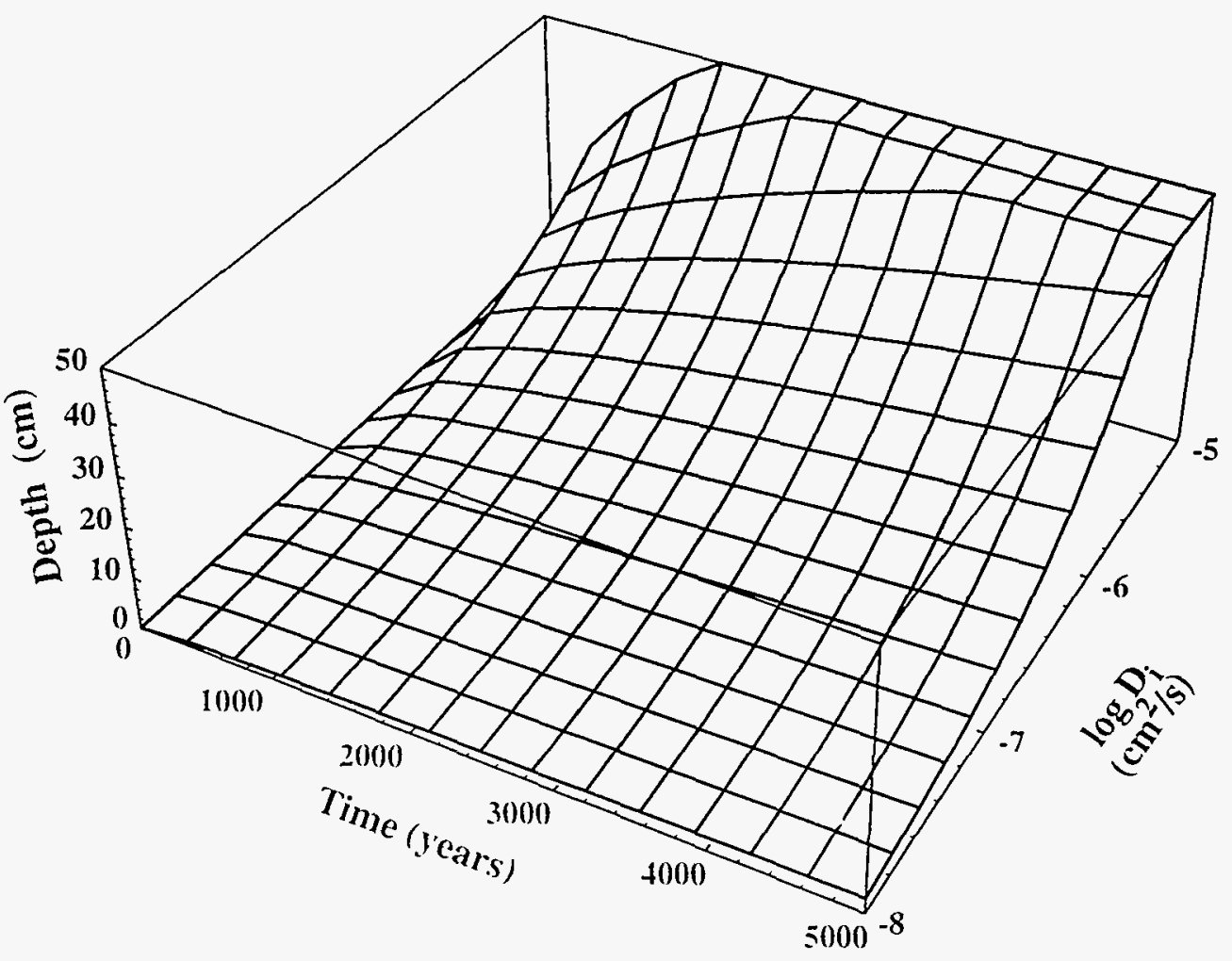

Figure 3. Depth of carbonation over a range of diffusion coefficients representing intact concrete to diffusion in water. 


\section{Steel Reinforcement Corrosion}

One of the properties of concrete that makes it an attractive material for use in low-level radioactive waste disposal structures is its high compressive strength. However. concrete has a relatively low tensile strength. Steel reinforcement is used in concrete to increase tensile strength of structures. The concrete cover over the steel reinforcement provides a protective environment from corrosive agents such as oxygen and chloride by creating a passivation layer around the steel. Breakdown of the passivation layer allows corrosion to occur. Which over time will reduce the tensile strength of the concrete structure and may result in structural instability. Also. the steel corrosion products have a greater molar volume than the solids they replace. This expansion can cause spalling or cracking of the concrete. thereby increasing the permeability of the concrete.

Prediction of steel reinforcement corrosion is conducted by viewing the time to breahup of the passivation layer (initiation of corrosion) and corrosion rate subsequent to breakup of the passa ation layer separately. An empirical model for time to onset of corrosion and a corrosion rate model are ured to calculate these processes.

Chloride Attack involves the disruption of the passive layer that has historically been associated with contacting the steel reinforcement with chloride ions (ACI. 1985). The chloride concentration that will lead to steel corrosion decreases with reduced alkalinity in the concrete because the passivation layer is less stable. Depassivation of steel reinforcement can occur when the $\mathrm{pH}$ near the rebar drops below 9 (Papadakis et al., 1989). As stated previously, carbonation and leaching are procenes that will lead to gradual lowering of $\mathrm{pH}$ and eventual loss of passivity. In low chloride environments, carbonation or leaching may be important processes in depassivation. For the Pennsylvania $\backslash$ aults. carbonation and leaching are not significant processes over the time frame requiring an intact hydrologic barrier. Decreasing the $\mathrm{pH}$ of the concrete below 9 generally takes on the order of thousands to millions of years because the processes that cause the $\mathrm{pH}$ to drop are slow (Walton et al., 1990).

The empirical model to predict the time to depassivation was developed by Stratful and modified by Clear (1976). Clear's study of steel corrosion in concrete slabs indicates a link between the time until depassivation with the depth of cover over the reinforcement and the water cement ratio. The chloride content of the contact water is also a factor in the estimating the time to once of steel corrosion (Equation 5). Potential limitations of this model are that the observations that were uned as a basis for the model are for corrosion of bridge decks and that soils do not typically have chlorde concentrations in this range. Also, the model does not consider a chloride threshold that would negalte corrosion at some low chloride concentrations (thought to be a conservative limitation).

The parameters required for this model are the concrete coverage over the steel reinforcement, the water to cement ratio. and the chloride concentration in the contact water. The concrete mu design and the vault design specify a water-to-cement ratio of 0.38 and a minimum steel reinforcement coverage of 3 inches. The chloride concentration used in the corrosion estimate (1.2+E- $+\mathrm{M}$ or $4.4 \mathrm{ppm})$ is taken from the water chemistry described above. 


$$
C L_{t}=\frac{129 \operatorname{cov}^{1.22}}{\| \mathrm{Crcl}_{g w}^{0 .+2}}
$$

$\begin{array}{ll}\mathrm{CL}_{l} & \text { time to onset of corrosion (year) } \\ \mathrm{cov} & \text { thickness of concrete over the rebar (inches) } \\ w \mathrm{cr} & \text { water to cement ratio (by mass) } \\ \mathrm{cl}_{\mathrm{gw}} & \text { chloride ion concentration in the groundwater (ppm) }\end{array}$

These model parameters were used as a basis to estimate time to onset of steel reinforcement corrosion. The time to onset of corrosion was evaluated by varying the chloride concentration from $10^{-4}$ to $10^{-2}$ moles $/ \mathrm{L}$ at the concrete coverage at the water-to-cement ratio listed above. Time to depassivation ranges from 700 to 100 years under these conditions (see Attachment I. Appendix B). The reference water composition for the evaluation is approximately $10^{-4}$ moles/ $\mathrm{L}$ indicating that depassivation could take a significant amount of time.

Time to onset of corrosion was also evaluated for a range of reinforcement depths from 2.5 to $20 \mathrm{~cm}$ ( 1 to 8 inches) within the concrete using the reference water chloride concentration. Results indicate that placing the reinforcement deeper into the concrete $(20 \mathrm{~cm}$ or 8 inches) would increase the time to onset of corrosion from 700 years to 2.400 years. Increasing the coverage would also provide additional time before leaching or carbonation could increase corrosion.

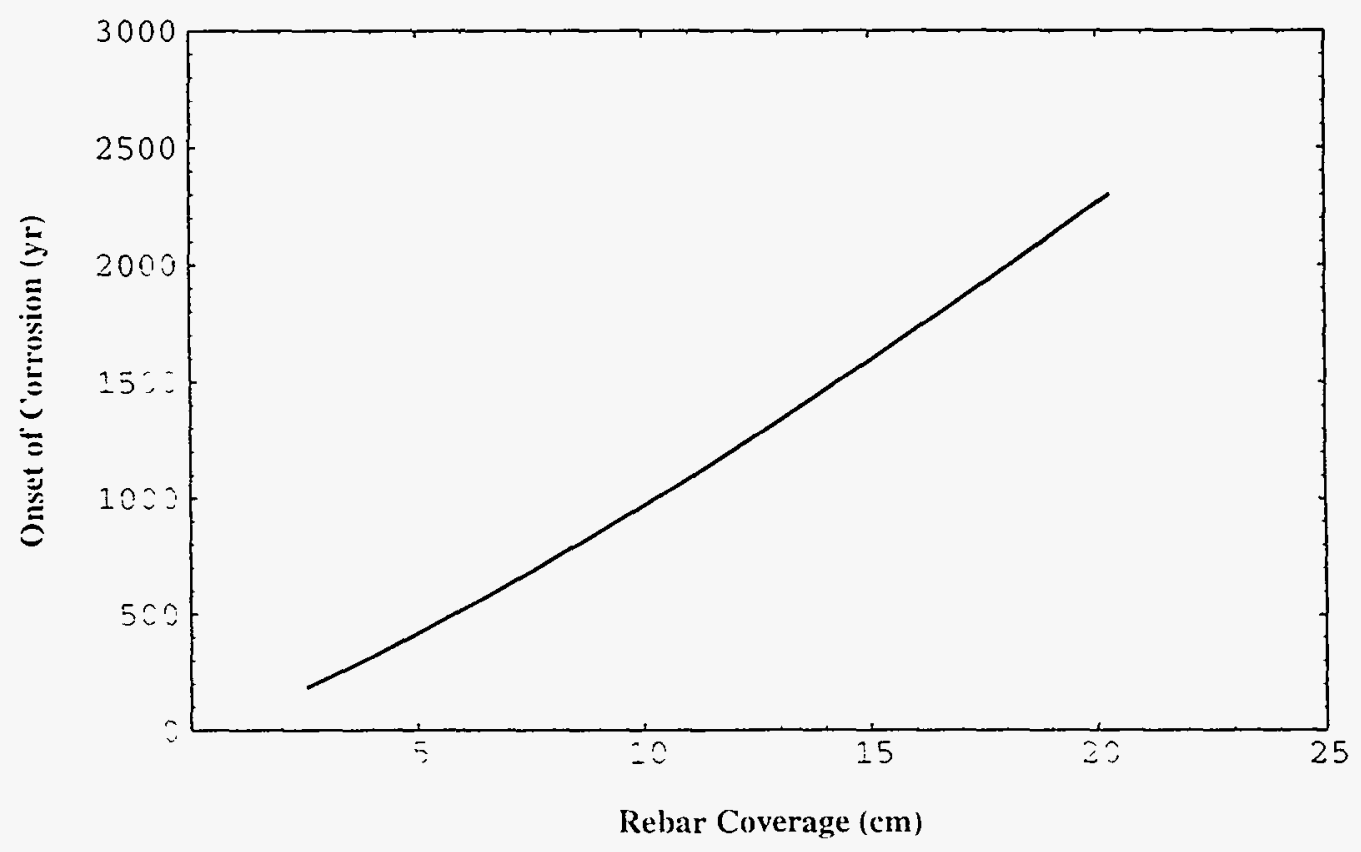

Figure 4. Time to onset of corrosion for variable coverages of steel reinforcement at $1.24 \times 10^{-4} \mathrm{M}$ chloride concentrations. 
Oxygen Diffusion Limited Rebar Corrosion can begin following the disruption of the passivation layer surrounding the rebar by chloride attack. The smoothest way of modeling corrosion of rebar is to assume that diffusion limits the supply of oxygen to the rebar for reaction. Corrosion is then a factor of the diffusion rate and contact water concentration of oxygen, the diameter and spacing of the rebar, and the coverage thickness of concrete over the rebar (Equation 6). The model does not account for the increased transport rate of oxygen to the rebar as the effective thickness of the concrete is decreased due to concrete degradation processes. This is not practical given the lack of site-specific data al ailable for use in the modeling. To compensate for this. the time to onset of corrosion is neglected and corrosion is initiated at the time of emplacement.

The rebar used in the modeling is assumed to be \#+ (0.5-inch diameter) in size on 1-foot spacing with 3 inches of concrete coverage. The oxygen concentration in water in equilibrium with the atmosphere is approximately $10 \mathrm{ppm}$ or $3.125 \mathrm{E}-4$ moles/Liter and will be used in the corrosion calculations because no other data are available. Several diffusion coefficients for oxygen in concrete have been used because this is the parameter with the most uncertainty.

$$
R R=\left(1-\frac{4 \times 9 \frac{c^{3} m^{3}}{m o l e} \operatorname{sr} D_{i} \sigma_{g u} t}{\pi d^{2} \operatorname{cov}}\right) 100
$$

$\begin{array}{ll}\mathrm{RR} & \text { percent rebar remaining } \\ \mathrm{cov} & \text { depth of reinforcement below surface }(\mathrm{cm}) \\ \mathrm{D}_{\mathrm{i}} & \text { oxygen diffusion coefficient in concrete }\left(\mathrm{cm}^{2} / \mathrm{second}\right) \\ \mathrm{O}_{\mathrm{gw}} & \text { concentration of oxygen in surrounding groundwater }\left(\mathrm{mole} / \mathrm{cm}^{3}\right) \\ \mathrm{d}^{3} & \text { diameter of reinforcement }(\mathrm{cm}) \\ \mathrm{Sr} & \text { spacing between reinforcement bars }(\mathrm{cm}) \\ \mathrm{t} & \text { time (seconds). }\end{array}$

Results of these calculations show that 4 percent of the rebar is lost using an intact concrete diffusion coefficient of $10^{-7} \mathrm{~cm}^{2} / \mathrm{s}$. A diffusion coefficient of $2 \times 10^{-6} \mathrm{~cm}^{2} / \mathrm{s}$ causes a significant increase in the degradation rate. At 1.000 years. only 40 percent of the rebar remains. The worst-cile xenario. which uses the free water diffusion coefficient. indicates that all the rebar will be corroded after 350 years. This is highly conservative and should be used only as an evimate of the upper bound. If we consider the time to onset of corrosion (700 vears), the amount of rebar remanning at 1.000 years is approymately 90 percent using a diffusion coefficient appropriate for degraded concrete $\left(2 \times 10^{-6} \mathrm{~cm}^{2} / \mathrm{s}\right)$. 


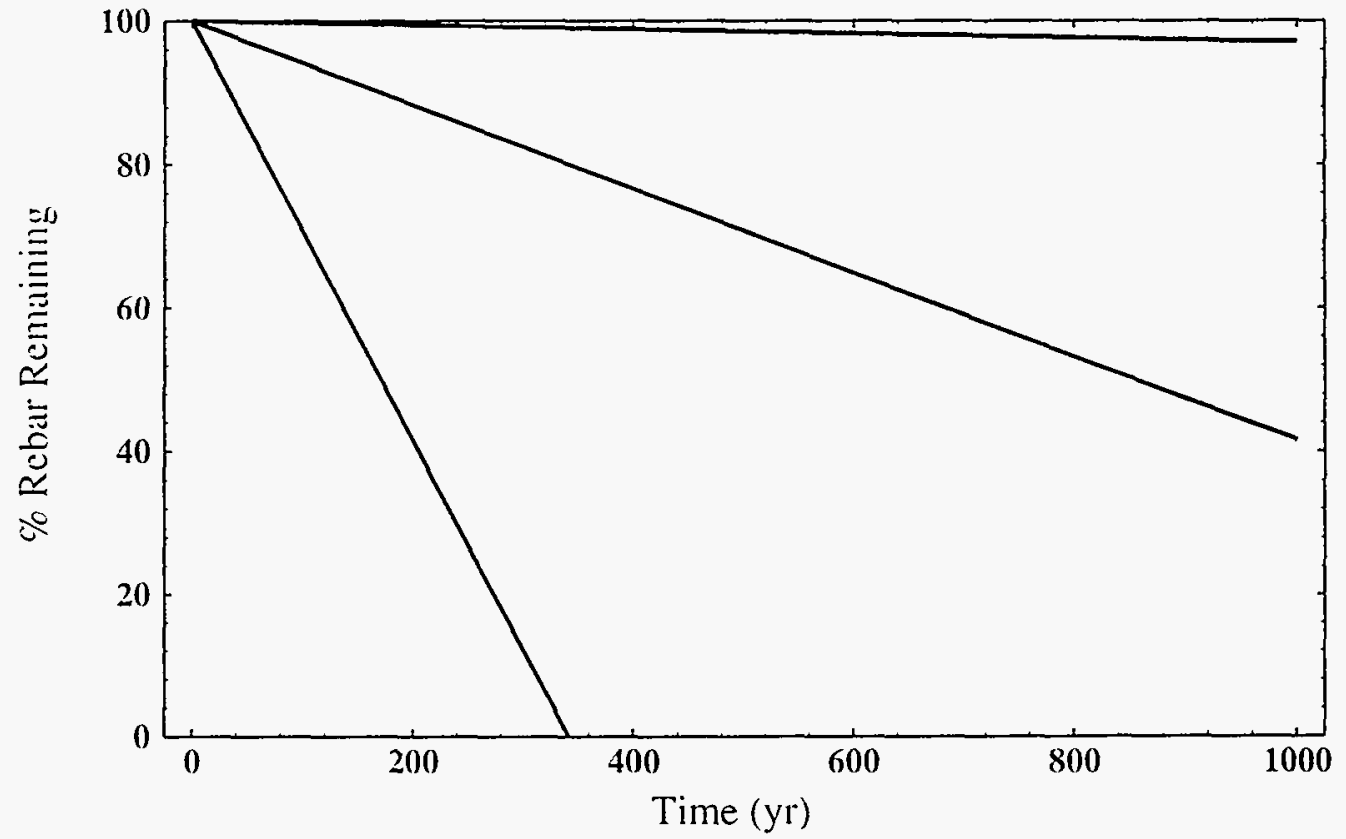

Figure 5. Percent steel reinforcement remaining considering three oxygen diffusion coefficients.

\section{Summary of Degradation and Corrosion Estimates}

Magnesium and sulfate attack, calcium leaching, carbonation of the concrete and rebar corrosion are not significant degradation processes in the "Proposed Environment." Based on these "Environmental" assumptions, the concrete vault shell will not be degraded due to any of the modeled processes for at least 1,000 years. Magnesium and sulfate attack has the greatest impact on the concrete and is predicted to reach the rebar in approximately 3.000 years. This would result in more rapid corrosion of the rebar, but will not impact the vault performance if the sault interior is backfilled.

Results indicate that moving the steel reinforcement deeper into the concrete would enhance the long-term durability of the vaults by keeping the passivation layer stable for a longer period of time. The concrete mix design might be modified after site selection to optimize the long-term performance of the vault.

\section{REFERENCES}

American Concrete Institute Committee 222. 1985. Corrosion of Metals in Concrete. ACI Journal, p. 3-32.

Atkin..on. A.. 1985. The Time Dependence of pH Within a Repository for Radioactive Waste Disposal, AERE Harwell Report No. DOE/RW/85.062.

Atkinson. A. and Hearne. J.A.. 1984. An Assessment of the Long-Term Durability of Concrete in Radioactive Waste Repositories. AERE-R11465. Harwell, UK. 
Atkinson, A., Ewart, F.T.. Pugh, S.Y.R., Rees. H.H.. Sharland. S.M.. Tasker. P.W.. and Wilkins, J.D., 1988. Experimental and Modeling Studies of the Near-Field Chemistry for Nirex Repository Concepts, Near-Field Assessment of Repositories for Low and Medium Level Radioactive Waste, Nuclear Energy Agency, OECD, Paris, France. p. 143-157.

Clear, K.C., 1976. Time to Corrosion of Reinforcing Steel in Concrete Slabs, Volume 3. Performance After 830 Daily Salt Applications. Federal Highway Adminıstration Report No. FHWA-RD-76-70. NTIS PB-258. p. 446.

Papadakis, V.G., Vayenas. C.G.. Fardis, M.N.. 1989. A Reaction Engineering Approakch to the Problem of Concrete Carbonation. AIChE Journal, Volume 35. No. 10. p. 16.39-1650.

Todd, D.K., 1980. Groundwater Hydrology.(Second Edition), John Wiley and Sons. New York, pp. 278.

Verbeck, G.J., 1958. Carbonation of Hydrated Portland Cement. PCA Research Department Bulletin 87.

Walton, J.C., Plansky. L.E., and Smith. R.W.. 1990. Models for Estimation of Service Life of Concrete Barriers in Low-Level Radioactive Waste Disposal. U.S. Nuclear Regulatory Commision report NUREG/ CR-5542, EGG-2597. 


\section{Appendix C}

Engineering Design File for Sorption Coefficients 


\section{E.TGINEERING DESIGN FILE}

Project/Task Pennsvlvania Performance Assessment Support

Subtask Distribution Coeficients Selection for PA's

EDF Page 1 of 13

\section{TITLE: Distribution Coefficients}

\section{SUMMARY}

This EDF documents the defintion of distribution coefficients $\left(\mathrm{K}_{d}\right)$ and how $\mathrm{K}_{\mathrm{d}}$ 's are determined by experimental methods or selected by literature review for use in Performance Assessmenis (PA). This document describes how disirbution coefficients are determined by batch and column experiments and how $\mathrm{K}_{\mathrm{d}}$ 's are selected for use in performance assessments The primary focus will be on selection $K_{j} s$ for use in PA and the important aspects to consider dunng the selection process. Contaminant movement is a funcuon of water movement through the subsurface and geochemical interacuons between the porous media. groundwater, and contaminant. The majonty of current iransport models depend upon $\mathrm{K}_{d}$ 's to predict the geochemical interactions between contaminants and porous media because it is the least complex numerical means of describing sorption. The $\mathrm{K}_{d}$ used in transport modeling is a bulk term that encompasses all processes that remove a contaminant from the solution and is defined as the amount of contaminant removed from solution per mass of solsd ( $S$ ) divided by the amount of contaminant in a volume of solution (C) (typical units are $\mathrm{mL} / \mathrm{g}$ ).

$$
K_{d}=\frac{S}{C}
$$

Distribution coefficients are used in all phases of the groundwater pathu ay analysis in PA. Screening calculations use $K_{d}$ 's to determine which contaminants are key to the performance of the facility. Soil or geologic material $K_{d}$

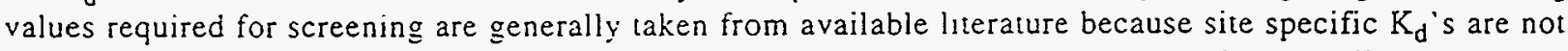
available. Key contaminants are modeled using a numerical flow and transport code to predict movement considering the wide vanety of materials associated with the disposal facility (e.g. grout/concrete, clay, sand and gravel). The equation that forms the basis for both screening and numerical contaminant movement evaluations is given below. More detailed evaluation of the $K_{d}$ is recommended for this portion of the PA and may require experimental determinations with site-specific materials.

$$
v_{c}=\frac{v_{w}}{1-\frac{\rho_{b}}{\theta} K_{d}}
$$

where $\quad \rho_{b}=$ bulk density of geologic material, $\theta=$ porssai of geologic matenal.

$$
P_{i}=\text { velocit! of the contaminant. } \quad v_{\mathrm{u}}=\text { velocity of the water }
$$

Controls on contaminant removal from solution can be separated into soilc characteristics and solution composition for field conditions. Laboratory expenments have additionai controls such as the solid solution ratio and contact time in addition to the field controls. Ion exchange, non-speciñc adsorption, specific adsorption, and precipltation are the mechan:sms that are associated with the solid phase charactenstics. Solution characteristics are inorganic or organic complexation and colloid formation that result in changes in solution speciation. A bnef descripuon of each mechanısm that contributes to the partitioning of contaminants is given in the EDF.

Disinbution icomplete package.: Disinbution isummary page only.
C.A. Dicke, R.S. Garcia. S O Magnuson, C.S. Smith, A.S. Rood

G.J. Stormberg, LITCO Project EDF File Log

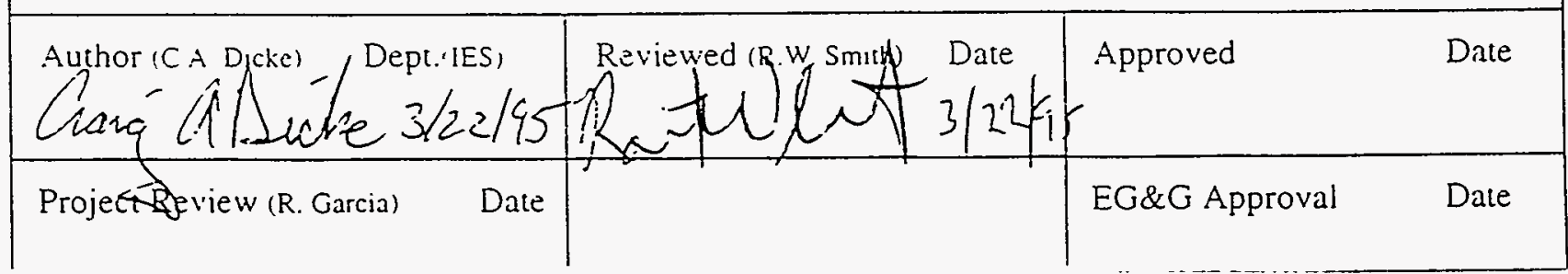




\section{DISTRIBUTION COEFFICIENTS IN PERFORMANCE ASSESSMENT}

Regulations for the siting and design of Low-Level Waste (LLW) facilities requires that the movement of contaminants in the local environment be addressed as part of a Performance Assessment (PA). Contaminant movement is a function of water movement through the subsurface and geochemical interactions between the porous media, groundwater, and contaminant. The majority of current transport models depend upon distribution coefficients $\left(\mathrm{K}_{\mathrm{d}}\right)$ derived from linear isotherms to predict the geochemical interactions between contaminants and the porous media. The distribution coefficient is defined as the amount of contaminant removed from solution per mass of solid divided by the amount of contaminant in a volume of solution (typical units are $\mathrm{mL} / \mathrm{g}$ ).

Distribution coefficients are used in all phases of the groundwater pathway analysis in Performance Assessment. Screening calculations use $\mathrm{K}_{d}$ 's to determine which contaminants are key to the performance of the facility (Seitz, 1995). Soil or geologic material $K_{d}$ values are required for this portion of the PA and are often taken from available literature because site specific $K_{d}$ 's are not available. The movement of key contaminants identified in the screening calculations are modeled using a groundwater flow and transport code to predict if regulatory limits will be exceeded. A more rigorous evaluation of the distribution coefficients used in this portion of the PA is often necessary. Also, a wide variety of materials associated with the disposal facility (e.g. grout/ concrete, clay, sand and gravel) as well as the local soil or geologic material will need distribution coefficients for transport modeling. These values are obtained from site specific experiments if available, literature review, or calculated using available information.

This document describes how distribution coefficients are determined by batch and column experiments and how $\mathrm{K}_{\mathrm{d}}$ 's are selected for use in performance assessments. The primary focus will be on selection $\mathrm{K}_{d}$ 's for use in PA and the important aspects to consider during the selection process. The Langmuir and Freundlich isotherms that are used in some transport codes to describe sorption will be briefly described in Appendix $\mathrm{A}$.

\section{Sorption in Contaminant Transport Modeling}

Contaminant mobility is one of the key criteria in evaluating performance assessment of Low-Level Waste (LLW) disposal facilities. Mobility of contaminants is generally incoreorated into transport

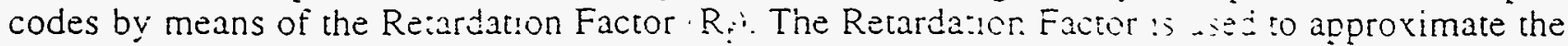
movement of contaminants in groundwate , Equation 1 ).

$$
R_{i}=1+\frac{\rho_{b}}{\theta} \frac{S}{C}
$$

where $\quad R_{i}=$ retardation factor,

$\rho_{b}=$ bulk density of geologic material,

$\theta=$ porosity of geologic material.

$\mathrm{S}=$ mass of contaminant adsorbed per unit mass or geologic material, and

$C=$ mass of contaminant in solution per unit voinme of solute.

The average velocity of a contaminant is then calculated based on the groundwater velocity and retardation factor (Equation 2). This estimate assumes that the contaminant concentration is much lower than the total concentration of cations in the groundwater (i.e. competition is not a factor). It is also important to remember that this calculation provides the location of the mean concentration in the plume and not the leading edge of the plume. 


$$
V_{c}=\frac{V_{w}}{R_{f}}
$$

where $\quad V_{c}=$ velocity of the contaminant,

$V_{\mathrm{w}}=$ velocity of the water, and

$R_{\mathrm{f}}=$ retardation factor.

This basic equation for predicting the contaminant velocity is used in the screening process discussea by Seitz (1995). For the screening process the distance to the receptor and groundwater velocity is used to determine the groundwater travel time. The radionuclide travel time (RTT) is estimated by Equation 3 as show'n in Seitz (1995). This method can be used for the waste inventory as discussed by Seitz (1995) to identify key contaminants.

$$
R T T=\frac{X}{V_{w}} R_{f}
$$

where $\quad$ RTT $=$ radionuclide travel time for mean concentration.

$\mathrm{X}=$ distance to compliance point.

$V_{w}=$ velocity' of the water, and

$\mathrm{R}_{\mathrm{f}}=$ retardation facior .

Inventories often contain many radionuclides (hundreds), some metals (tens), and miscellaneous organic and inorganic constituents (tens). Ideally sorption data would be available for all contaminants in the inventory for site conditions. This is unlikely due to the cost and time involved in conducted this level of experimentation. Therefore, it is usually necessary to use $\mathrm{K}_{d}$ 's from literature to establish a list of contaminants that represent the most risk in the performance of the facility. The lack of data during the screening phase of Performance Assessments precludes the use of more refined isotherms, but this is generally not a problem because the difference between linear and non-linear isotherms is minimal under normal environmental conditions (Sheppard and Thibault, 1990). It is recommended that values from Sheppard and Thibault (1990) be used for screening purposes becalise the distribution coefficients should represent generic conditions assumed at this point in the PA process.

Groundwater pathway calculations for Performance Assessment are also based on Equation 2 for estimating the contaminant mobility in the numeric models. The numerical modeling requires distribution coefficients for many iypes of adsorbents. Construction of Low-Level Waste disposal facilities often involves the use of concrete. Concrete is a very good zorting environment for may radionuclides. particularly actinides. Also. the concrete mix can be adjusted to optimize the sorption capability for mobile constituents like technetium. Addition of materials like blast furnace slag and fly ash that create reducing conditions in the concrete will cause reduction of the technetium to a less soluble phase. Clay and gravel may also be used in the construction of the drainage system at the disposal facility. Distribution coefficients for these materials is often taken from the literature. Site specific sorption parameters may need to be determined for specific contaminants for the soil at the facility location. This can be kept to a minimum by conductirg sensitivity calculations using the flow and transport code.

\section{Factors That Influence Distribution Coefficients}

One of the major problems with the use of $\mathrm{K}_{d}$ 's in modeling is that variations of several orders of magnitude have been measured for a site-specific material with no insight into the reason for the variation (Kent et al., 1988). Controls on contaminant removal from solution can be separated into 
solid characteristics and solution composition for field conditions. Laboratory experiments have additional controls like the solid solution ratio and contact time in addition to the field controls. Ion exchange, non-specific adsorption, specific adsorption, and precipitation are the mechanisms that are associated with the solid phase characteristics. The sorption mechanism that controls contaminant removal is dependent on the solid types that are present in the solid material. Removal of contaminants from solution by these mechanisms also depends on the form that the contaminant takes in solution which is a response to the composition of the solution. Solution speciation can effect the removal of contaminants from solution by decreasing the availability of the contaminant form that could be removed from solution to another form that will remain in solution (e.g. $\mathrm{UO}_{2}$ complexing to $\mathrm{UO}_{2} \mathrm{CO}_{3}$ ). Three mechanisms, inorganic or organic complexation and colloid formation, result in changes in solution speciation (Figure 1). A brief description of each mechanism that contributes to the partitioning of contaminants is given below.

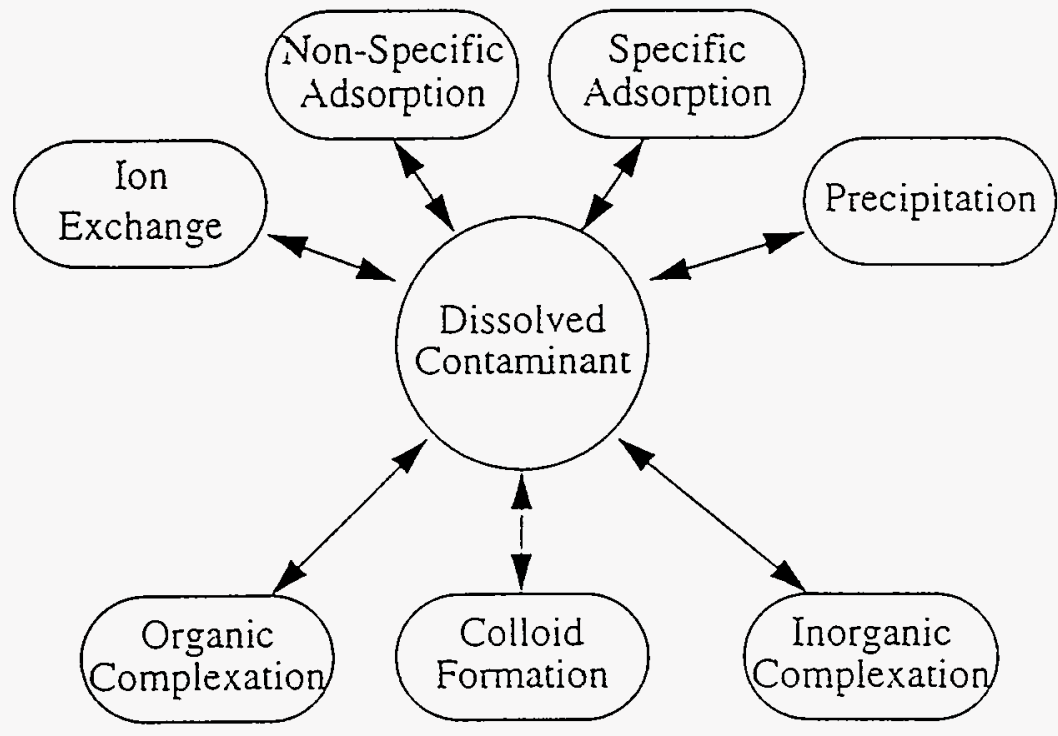

Figure 1. Mechanisms that control solid/solution partitioning of contaminants.

Ion Exchange: Fixed charge sorption slies will have an ion of opposite charge associated with it. Changes in the solution composition wan cause the ions in these exchange sites to be replaced by ocher ions based on concentration and affinity of the available :ons. These reactions are not tied to $\mathrm{pH}$ as are the non-specitic acisorbed ions because the charge is $:$ ixed. The cation exchange capacity, $\mathrm{CEC}$, is a measure of a material's capacity to bind cations. These types of bonds are more easily broken and the cation is then mobilized due to changes in the solution chemistry.

Non-Specific Adsorption: Vion-specific adsorption occurs to compensate for charges associated with specifically adsorbed ions. The ion exchange is conceptualized to occur between the solution and the specifically adsorbed ions due to adjustments in the electrostatics brought on changes in solution chemistry. These types of bonds are associated with variably charged surfaces and form to neutralize the overall surface charge (i.e. surface charge plus non-specific charge equals zero). Most porous media have a net negative charge which results in preferential sorption of cations.

Specific Adsorption: This type of sorption involves both physical and electrostatic binding to the solid surface. The adsorbent is positioned closer to the solid surface than occurs in ion exchange reactions and the bond is stronger. 
Precipitation: Removal by precipitation can occur within the solution or on the solid surface. Precipitation occurs due to the reaction of two solution components when present in concentrations that exceed the solubility product for the reaction. Surface precipitating occurs when the concentration of a contaminant on the solid surface builds to a high level and precipitates on the solid surface.

Organic Complexation: Organic matter in soils can serve as a removal mechanism for contaminants and will therefore contribute to the $\mathrm{K}_{\mathrm{d}}$. Another issue that must be considered in many Performance Assessments is the presence of man-made organic compounds in the inventory. These compounds are generally solvents (e.g. trichloroethylene, carbon tetrachloride, and trichloroethane) used in materials processing and decontamination activities (e.g. citrate and ethylenediaminetetraacetic acid (EDTA)). Compounds such as EDTA form strong bonds with many cation and do not interact with solids because the charge of the complex is balanced.

Inorganic Complexation: Most metals and radionuclides will interact with solution components to some degree. Formation of hydroxide. carbonate. sulfate, chloride or nitrate species is common due to the higher concentration of these anions in solution. Often the sorbing phase of a contaminant is either a free ion or a hydroxide complex which is a function of the solution $\mathrm{pH}$. Formation of other complexes will then decrease the concentration of contaminant in the form that will sorb resulting in higher mobility of the contaminant (i.e. lower $\mathrm{K}_{d}$ ).

Colloid Formation: A colloid is a substance made up of particles that are larger than molecules, but are too small to be seen with an optical microscope (approximately 1 to $200 \mathrm{~nm}$ ). These particles can be either positively or negatively charged and will attract oppositely charged ions to neutralize the charge. Colloids can be an important transport process because contaminants can attach to the colloid and neutralize the charge of the particles. The particles will then move at approximately the speed of water with little or no interaction with the porous media.

\section{Solid Material Characteristics}

The mechanism or mechanisms that control sorption is dependent on the solid phases present in contact with the solution. The mineralogy of the geologic material or soil that control sorption include primary and secondary crystalline minerals and amorphous coatings such as hydrous iron, manganese. and aluminium oxides. Minerals important in sorption can be broken into four general categories (oxides. multiple site-type oxides, fixed charge, and salt type) based on the type of adsorption sites available on the solid. Secondary minerals and amorphous phases tend to dominate sorption when present even when present at low concentrations in comparison to mass of primary minerals. This is due in large part to the very high surface areas of these types of minerals.

Oxides have been characterized more extensively than any other of adsorbent type. Common minerals that are included in this category are: gibbsite, ferrihydrite, hematite, goethite, organic matter. cristobalite, opal, and quartz. These minerals are present in sediments and rock either as primary or secondary minerals (Kent et al. 1988). An important characteristic of these minerals is that their adsorption sites are surface hydroxyl groups that are amphoteric (i.e. act as acid or base by adsorbing protons in response to changes in $\mathrm{pH}$ ). At low $\mathrm{pH}$ the sorption sites will have a high concentration of protons making the surface charge positive. At high $\mathrm{pH}$ protons leave sorption sites to come into equilibrium with the solution. Therefore, cations will sorb more readily on oxides in higher $\mathrm{pH}$ solutions because the surface charge will tend to be negative. 
Multiple site-type oxides have not been characterized to the extent of simple oxides. These minerals exhibit both variable and fixed charge types making them more difficult to characterize. Some common minerals from this type of solid are: ilmenite, orthoclase, plagioclase. pyroxene, sanidine. zircon. Many of these minerals are feldspars formed as primary minerals and do not have the sorptive capacity of their alteration products (Kent et al., 1983). The fixed negative charge of these minerals is generated by the $\mathrm{SiO}_{4}$ and $\mathrm{AlO}_{4}$ tetrahedra framework that forms the basis of the feldspar minerals. The interstitial sites of the feldspars are filled by $\mathrm{Ca}^{2-} . \mathrm{va}^{+}$, and $\mathrm{K}^{+}$ions that balance the negative charge. Fixed charge at the surface occurs when one of the interstitial carions is removed from the solid leaving a negatively charged site to be filled. Hydration of surface layers may result in the formation of surface hydroxyl sites of variable charge as with the simple oxides.

Fixed charge minerals have sorption sites that remain negative even with changes in soiution composition and the sorption of contaminants on these minerals is controlled by ion exchange. Many of the minerals of this type are secondary minerals with open crystal strucrures. high surface area, high ion-exchange capacities, and high sorption capacities making them important in porous and fractured media. Examples of tixed charge minerals include: epidote, g! ass. biorite. chlorite. kaolinite, smectites, and zeolites.

Sorption related to salt type minerals is different from the mineral types listed above in that the solid coordination is a cation to anion bond as apposed to a cation to 4 or six oxide cons (Kent et al., 1988). Sorption may result from exchange of cations or anions between the solid and solution (e.g. $\mathrm{Sr}^{2+}$ replaces $\mathrm{Ca}^{2+}$ at the calcite surface). Examples of these minerals are: apatite, calcite, and dolomite.

It seems reasonable to assume that surface area would be a major controlling iactor in the uptake of contaminants by solids, but this is not always the case as shown in Sheppard and Thibault (1990). They provide a breakdown of distribution cesfificients for a wide varnety of eiements for the main soil types (i.e. sand, loam, clay, and organic ricn) and found that increased distrioution coefficients are not the rule for decreasing grain size (increased surface area). This is possibly a result of not looking at the mineralogic aspect of the sorbing material. Wide variations in sorption can be accounted for by minimal concentrations oi highly reactive minerals such as oxides (e.g. iron, manganese. aluminum, and silica) and clays ie.g. smectites, zeolites, illite, kaolinite, etc.). This information is often unavailable. Also, oxide contents of only several percent can control the

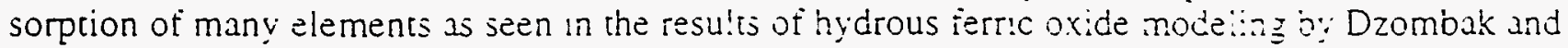
.Morel (1990).

\section{Solution Characteristics}

Water properties that impact the sorption of contaminants are: pH. Eh. temperazure. dissolved organic content, major cation concentration $\mathrm{Ca} . \mathrm{Mg}$, . $\mathrm{Va}, \mathrm{K}$ ). major anıon concentration $1 \mathrm{Cl}^{\mathrm{H}} \mathrm{HCO}_{3}$, $\mathrm{CO}_{3}, \mathrm{SO}_{4}$ ), and minor constrtuents such as naturaliv occurring stable isotopes of $\mathrm{Sr}, \mathrm{C}$, and I. All of these properties affect the speciation of solution components as discussed in general terms above.

The $\mathrm{pH}$ of the solution will impact the sorption of a contaminant in several ways. Solids with variable charged sorption sites in contact with the solution $w_{11}$ equilibrate to changes in the solution $\mathrm{pH}$. This may result in increased or decreased sorption depending on the charge of the contaminant and the change in the $\mathrm{pH}$. Also. changes in the $\mathrm{pH}$ will lead to changing speciation of solution components. Increasing $\mathrm{pH}$ may lead to formation of hydroxide species that do not sorb to solids in contact with the solution or precipitation of carbonate species may become important. In general cations sorb better as $\mathrm{pH}$ is increased. 
The redox potential symbolized by Eh measures the tendency of a solution to allow a reaction to proceed. Therefore. this potential is important for elements that can exist in more than one oxidation state such as technetium. chromium. uranium. plutonium, neptunium, and americium. Elements such as technetium and chromium that are insoluble in reducing conditions are very soluble in their oxidized state. The other radionuclides listed can exist in as many as four oxidation states. Each oxidation state may sorb to different degrees because the controlling mechanism may change with the oxidation state.

Temperature variations are not as critical for sorption in low-level waste storage facilities because the waste package does not generate the heat as seen in the high-level waste program. Organic content can impact sorption by complexing with the contaminant thereby reducing the concentration in solution. Organic material may not be part of the solid matrix, but is present as fine particles that will move with the water. Sorption onto this material will provide a means of rapid transport with minimal interaction with solids in the flow path. This is essentially colloidal transport.

Solution composition can impact sorption dramatically. Generally this is reflected by the major cation ( $\mathrm{Ca}, \mathrm{Mg}, \mathrm{Na}, \mathrm{K}$ ) and anion $\left(\mathrm{Cl}, \mathrm{HCO}_{3}, \mathrm{CO}_{3}, \mathrm{SO}_{4}\right.$ ) concentrations. High concentrations of cations can reduce sorption of positively charged contaminants by competing for available sorption sites. Anıon concentrations may result in complexation of positively charged contaminants in forms that will not sorb. Precipitation of contaminants as carbonate or sulfate species will remove contaminants from solution. This is the one of the reasons why the solution composition should be considered in selecting $\mathrm{K}_{d}$ 's from literature. The concentration of the contaminant itself is also important because in general the $\mathrm{K}_{\mathrm{d}}$ decreases with increased contaminant concentration due to competition for adsorption sites.

\section{Experimental Sorption Conditions}

The experımental conditions used to generate a distribution coefficient should also be evaluated to determine that the experiment is applicable to the field conditions. The contact time used in the experiment should be long enough to ensure equilibrium is attained. This requires experimental work to determine the time period required for equilibrium. A senes of sorption experiments are run under the same conditions for differing time intervals. Plotting the concentration sorbed versus time should vield a curve the flattens out at a specific time. After this point equilibrium is assumed for the experiment.

The solid solution ratio used in the experiment can also impact the $\mathrm{K}_{\mathrm{d}}$ produced. This will be seen as decreased sorption as the at:o decreases for the same contaminant concentration due to competition. The decrease is not always seen because it is also a function of the sorption capacity of the solid in comparison to the concentration of the contaminant. Consideration should be given to the solid/solution ratio used in expermments versus what is present in the field. in most cases this is significantly different.

Batch experiments often use a soll/solution ratio of 1 gram of adsorbent to $10 \mathrm{~mL}$ of solute. This does not represent the conditions seen in the field which are more in line with $50 \mathrm{~g}$ of soi', to $10 \mathrm{~mL}$ of solution. Assuming 50 percent saturation in a soil results in a ratio of $100 \mathrm{~g}$ of soil to $10 \mathrm{~mL}$ of solution. Column experiments provide a more realistic view of transport, but are often not practical because of the long duration of experiments involving moderate to highly reactive contaminants. 


\section{Distribution Coefficients and Sorption}

For the purposes of this report the term sorption is used to describe all processes that result in removal of a contaminant from the aqueous phase. Adsorption refers to an actual chemical process that bonds contaminants to a solid. The distribution coefficient $\left(\mathrm{K}_{\mathrm{f}}\right)$ used in transport modeling is a bulk term that encompasses all processes that remove a contaminant from the solution. This coefficient results from fitting a Linear Isotherm to experimental results and neglects the actual mechanism or mechanisms responsible for the contaminant removal.

The distribution coefficient is the least complex numerical means of describing sorption and for this reason has been widely incorporated into transport codes. Distribution coefficients are determined by measuring the amount of a known contaminant that remains in solution aiter contact with a known mass of a solid (Equation 3). The contaminant $\mathrm{K}_{\mathrm{d}}$ is then determined by simply recasting Equation 3 (Equation 4).

$$
S=K_{i} C
$$

or

$$
K_{d}=\frac{S}{C}
$$

where $\quad S=$ mass of contaminant adsorbed per unit mass of geologic matenal.

$\mathrm{C}=$ mass of contaminant in solution per unit volume of solute, and

$\mathrm{K}_{\mathrm{d}}=$ distribution coefficient.

Several shortcomings must be kept in mind when using $K_{d}$ 's for Performance Assessments (P.A) and other modeling activities. First. $K_{d}$ 's partition the contaminant into the solid and liquid phases with no upper limit to the amount of contaminant that can be sorbed. This implies that there is an infinite number of adsorption sites in the soil or geologic material. In dilute plumes this assumption is generally not a problem because the number of adsorption sites will be numerous in relation to the contaminant concentration. Experiments should be conducted to determine the contaminant concentration that causes the linear relationship to breakdown (Figure 2). Nonlinear experimental results require a different isotherm like the Freundlich to describe the sorption ' See Appendix A).

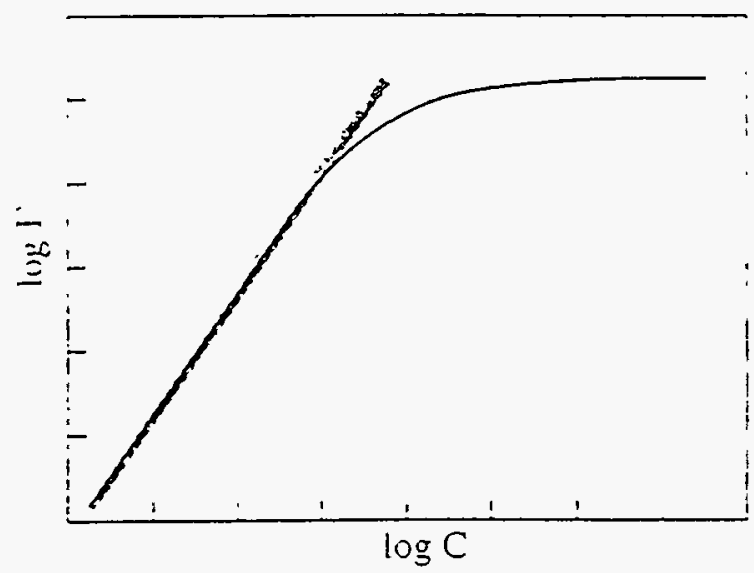

Figure 2. Characteristic adsorption isotherm. Linear portion of the plot can be described by a Kd. To caprure the information in the remander of the plot another isotherm like the Langmuir Isotherm. 
An example of an adsorption isotherm is given in Figure 2 where $C$ represents the concentration of the contaminant in moles $/ \mathrm{L}$ and $\Gamma$ is the site density in moles/mole. The gray zone represents the portion of the isotherm that can be described by a $K_{d}$ (i.e. the relationship is between $C$ and $\Gamma$ is linear). The line begins to flatten because the concentration of available adsorption sites are becoming limited. Often a series of isotherms are produced for different $\mathrm{pH}$ conditions in order to determine the sensitivity of the contaminant to $\mathrm{pH}$ variations.

Distribution coefficients range from $0 \mathrm{mLig}$. no sorption. for conservative contaminants like tritum to 100's of thousands for reactive contaminants on high surface area materials (e.g. plutonium sorption on tuff). It is important to keep the amount of contaminant actually being removed from solution in perspective particularly with high $\mathrm{K}_{\mathrm{d}}$ values (Figure 3). Almost all of the contaminant is sorbed by the time the $\mathrm{K}_{\mathrm{d}}$ reaches a value of $100 \mathrm{~mL} / \mathrm{g}$. After this point the relative gain in percent sorbed is very low in comparison to the $K_{d}$ value. However, the $K_{d}$ range above $100 \mathrm{~mL} / \mathrm{g}$ can be significant when dealing with very long lived radionuclides that have high dose conversion factors (i.e. represent a significant hazard in small quantuties for extended periods of time).

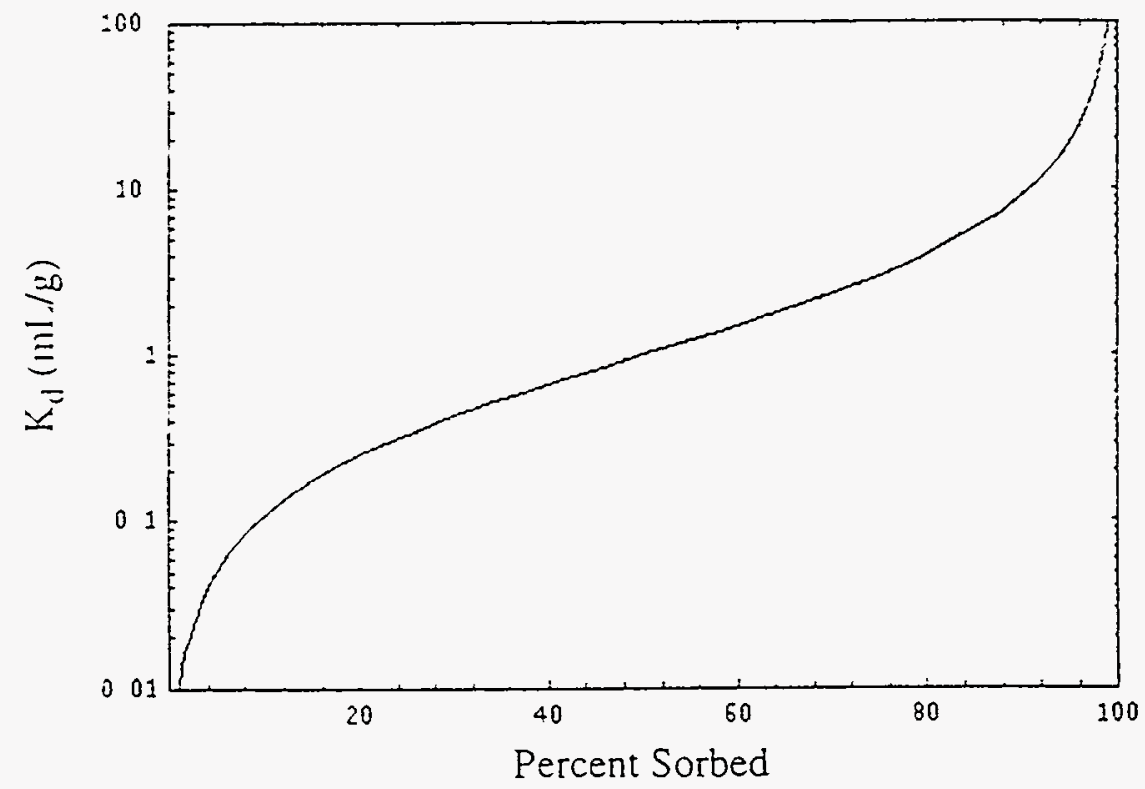

Figure 3. Relationship of distribution coefficient to the percent of contaminant sorbed.

\section{Distribution Coefficient Determination}

A variety of methods can be used to determine the distribution coefficient for a specific contaminant, solution and solid. Batch experiments are the most predominant method because results can be obtained relatively quickly at reasonable costs for most contaminants. Column experiments require more time to setup and can take extensive time periods to complete for reactive contaminants (i.e. achieve breakthrough of the tracer). In situ studies are potentially the most userul, but are not often feasible due to costs and regulatory constraints. Estimating a $\mathrm{K}_{d}$ using existing values for non-site speciric materials is the least favorable method. 


\section{Experimental Methods}

The first requirement in conducting batch or column experiments is to determine the procedure to be used in the investigation. This entails determining the appropriate mineralogy, solution composition, solid/solution ratio (batch). contact time (batch) or flow velocity (column), particle size, temperature, and atmospheric conditions. The preparation of the solid and solution including the contaminant concentration to be used must also be determined. The first part of defining the procedures should involve some basic modeling of the system to evaluate possible complicating factors in the experiment such as precipitation of the contaminant. When actinide or other elements that can be present in more than one oxidation stace are the targeted ccntaminant it is critical to prepare the contaminant correctly to ensure that oxidation states are maintained.

\section{Batch}

Solid Preparation: Batch experiments are conducted by contacting the solid phase of interest with a contaminant in the selected solution chemistry. The solid phase is ground or crushed if it is consolidated material and the material is sieved to collect the size distribution of interest in the study. Typically the fine grained particles are removed from use in the batch experiments. The remaining solid is then contacted with the water selected for use in the batcn experiment to bring the freshly ground sample to equilibrium with the water composition. A split sample from the solid material is often used to equilibrate a water sample with the solid. Both types of samples are then centrifuged to separate the phases and the equilibrated water is also filtered to ensure no fine materials are included in the water. The filtered water is then used to prepare the tracer solution for the batch experiment.

Experiment: The prepared solid and tracer solution are then mixed at the spectried solid solution ratio. Solution volumes of between 10 to $50 \mathrm{ml}$ are often used in contact with 1 gram of solid. Batch experiments are usually conducted in either polyethylene or polycarbonate tubes with sealed tops. The tube or group of tubes are placed on a shaker and agitated for the required contact time. The tubes are then centrifuged and the liquid is extracted and the soluble contaminant concentration is determined. This procedure is followed for all solid/solution ratios and solution compositions of interest.

\section{Column}

In order to conduct a usetul column experment it is necessary to have an uncersianding of system that is being investigated. Therefore, batih experments are conducted z:: or :0 the column experiment to determine if the tume period required to conduct ine experiment is reasonable ti.e. weeks or months and not years I. Hignly sorptive materlals and reactlve contamsnants will not show contaminant concentrations i i.e. breakthrough) :or extensive periods of time and are not practical for column studies. Column experiments are particularly useful in determining retardation of contaminants that do not show appreciable sorption on a solid because the breakthrough of the contaminant can be monitored more precisely.

Solid Preparation: The preparation for column experiments is simllar to batch experiments. Unconsolidated material will be sieved and the grain size distribution of interest will be collected and contacted with the solution water to bring the water and solid into equilibrium. Unconsolidated material is packed in the column in lifts at specified compaction pressures to obtain the hydraulic properties that are of interest. Core samples may also be used in column experıments, but may be restricted to relatively permeable materials in order to obtain results in a reasonable time frame. Consolidated materials may also be ground or crushed and used in column experiments. In these 
experiments the ground material must be equilibrated with the solution composition prior to addition of the tracer.

Experiment: Column experiments begin by saturating the column with the water composition of interest. This should be done by gradually pushing water up through the bottom of the column to reduce the potential of air pockets forming within the column. After the column is saturated the flow field is established in the column and the contaminant is added after steady-state conditions have been achieved. The effluent water is sampled at prescribed iniervals for contaminant analysis and the resulting piot of time versus concentration will allow distribution coefficient to be determined using Equations 2.3. and 4.

\section{Literature Review}

Several compendia of distribution coefficients from the literature (Sheppard and Thibault, 1990: Coughtrey et al. 1985: Ames and Ra1. 1978: Isherwood. 1981) and a database containing $K_{d}$ 's for a wide variety of geologic material. single mineral phases and solution compositions (Ticknor and Ruegger. 1989) are available. A literarure review should first focus on any sorption work that has been done to characternze the facilit; site. However, site-specific information is often not available particularly during the screening phase of a PA. Lacking site specific information the next approach is to use the available literature values after comparing the experimental conditions (e.g. mineralogy, solution composition, contaminant concentration, etc.) of the literature value with site conditions. A more detailed description of the affects of solid and solution characteristics is provided in the next section. Most of the compendium's do not detail the experimental conditions and only give $\mathrm{K}_{\mathrm{d}}$ ranges and a suggested value for a given contaminant.

Sheppard and Thibault (1990) have used soil grain size classifications and organic content as a means of further separating their $\mathrm{K}_{d}$ listing. The NEA database (Ticknor and Ruegger, 1989) provides mineralogic and water chemistry data for each $\mathrm{K}_{d}$ in the listing. This provides the user with the opportunity to try to match site conditions when selecting a $\mathrm{K}_{\mathrm{d}}$. If no site specific information is available a generic approach must be taken. For this it is recommended that the sand values of Sheppard and Thibault (1990) be used. These values should be conservative for most conditions.

\section{References}

Ames. L.L.. and Rai. D., 1978. Radionuclide interactions with Soil and Rock Media, Volume 1 and 2. Las Vegas, NV, L'S. EP.A. Office of Radiation Programs: EPA 520/6-78-007-b.

Coughtrey. P.J.. Jackson. D.. and Thome. M.C.. 1985. Radionuclide Distnibution and Transport in Terrestrial and Aquanc Ecosystems: A Compendium of Data. Netherlands: A. A. Balkema.

Isherwood, D.. 1981. Geoscience Data Base Handbook for Modeling a Nuclear Waste Repository, Washington. D.C.: L.S. NRC: NLREG/CR-0912, Volume 1, LCRL-52719, Volume 1.

Kent. D.B. Tnpathi. V.S., Ball. A.B., and Leckie. J.O.. 1988. Surface-Complexation Modeling of Radionuclide Adsorption in Subsurface Environments, NUREG/CR-4807, SAND86-7175, 113 p.

Langmuir. I. 1918. The Adsorption of Gases on Plane Surfaces of Glass, . Mica and Platinum, Journal of Amencan Chemistry Society, Volume 40. pp. 1361. 
Onishi, Y.. Seme, R.J.. Amold, E.M., Cowan. C.E., and Thompson. F.L., 1981. Critical Review: Radionuclide Transport, Sediment Transpor, and Water Quality .Wathematical .Modeling; and Radionuclide Adsorption/Desorption .Mechanisms, NUREG/CR-1322, PNL-2901, Richland, WA.

Seitz, R.R., 1995. Screening Calculations for Hypothetical LLW Disposal Facility - Drinking Water Pathway. Numberless LITCO EDF. p. 25.

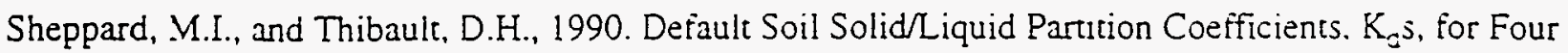
Major Soil Types: A Compendium. Health Physics, Volume 59, no. 4, pp. $4,1-432$.

Ticknor, K.V., and Ruegger. B.. 1989. A Guide to the .VEA's Sorption Data Base. version 2.0. (Septemuer), $19 \mathrm{p}$. 



\section{Appendix D}

\section{GWSCREEN Model Simulation Output}

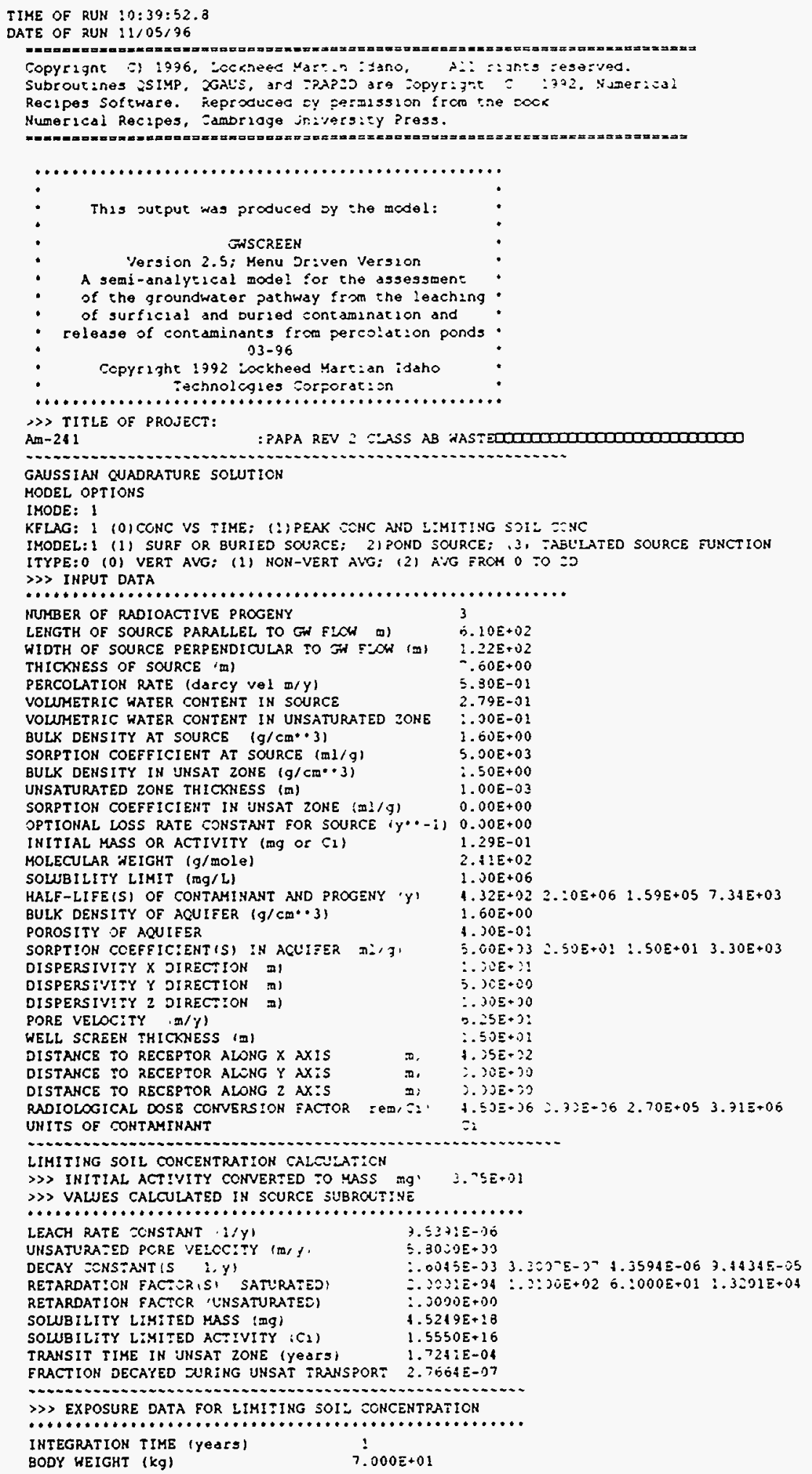

D-1 


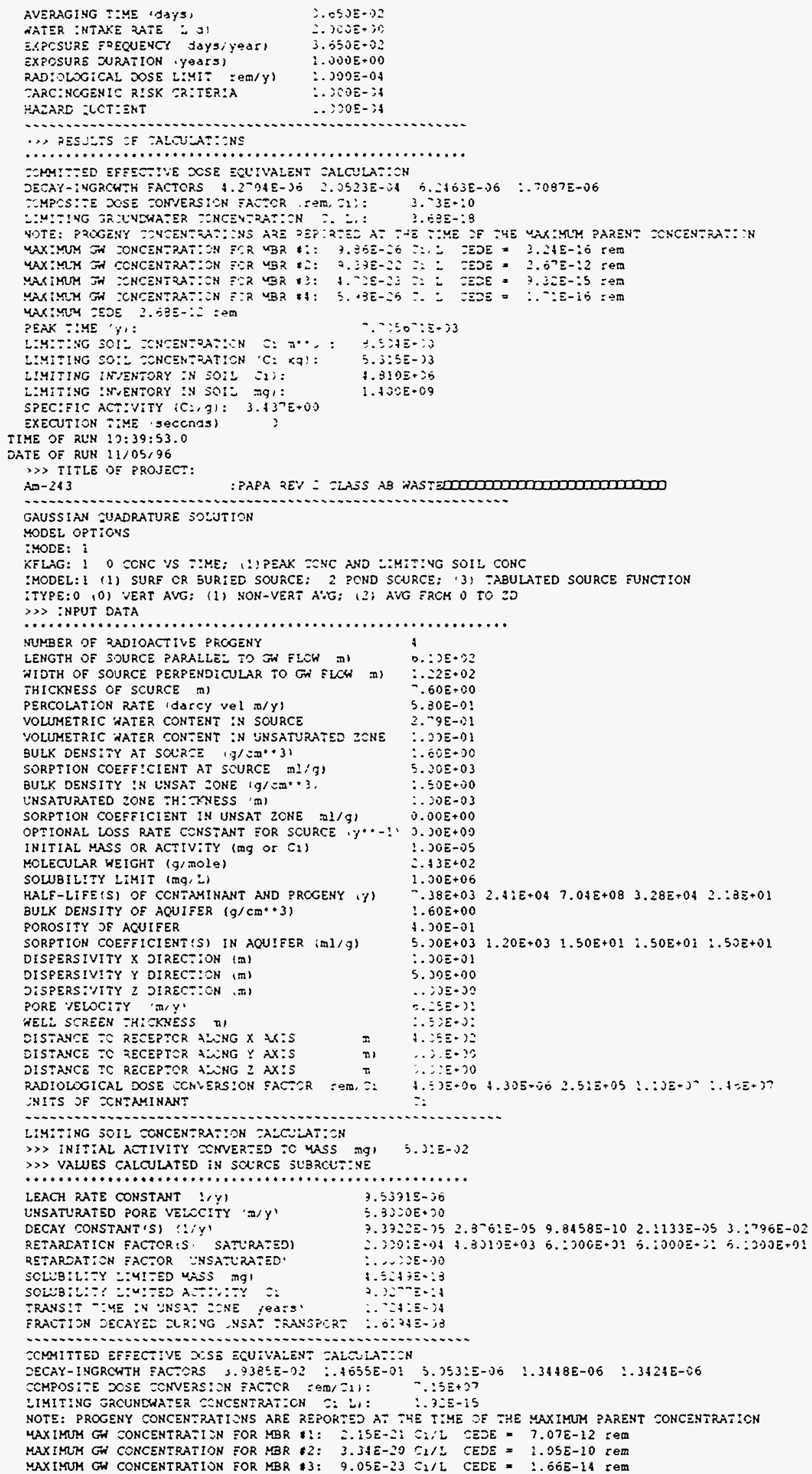




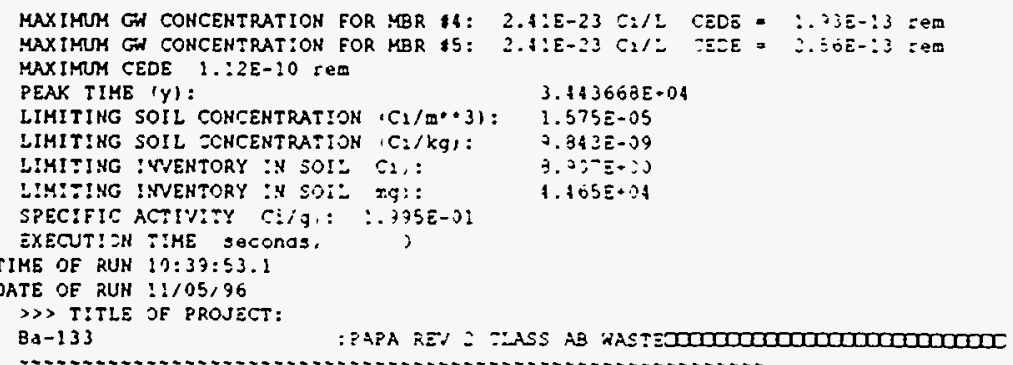

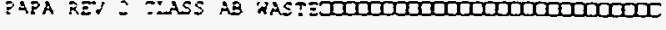

GAUSSIAN QUADRATURE SOLUTION

MODEL OPTICNS

IMODE: 1

KFLAG: 1 (0)TCNC US TIME: : PEAK ZONC ANC L:MIT:NG EO:Z ZOHE

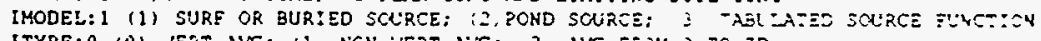

ITYPE: 0 (0) VERT AVG; 12 . NCH-JERT AVG; 2 AVG ERLM; :O 23

$\gg$ INPUT -ATA

MUMBER OF RADIOACT:VE PROGENT

LENGTH OF SCURCE PARAEIEL TO $\mathrm{G}$ :LLW $\mathrm{im}$

WIDTH OF SOURCE PERPENDIOJIAR TO $\mathrm{FN}$ ELON $: \mathrm{ml} \quad 2.22 \mathrm{I}+02$

THICKMESS OF SOURCE (m)

PERCOLATION RATE (darCY VOL $\mathrm{m} / \mathrm{Y}$ ) $5.80 \mathrm{E}-0$

VOLUMERIC HATER SONTENT :N SOLRCE 2.73E-O

VOUMETRIC WATER GCNTENT IN JNSATURATEO ZCNE 1.JOE-;

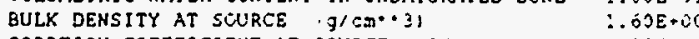

SORPTION COEFFICIENT AT SOURCE $\mathrm{mi} / \mathrm{gl} \quad: .00 \mathrm{E} \cdot \mathrm{O}$

BULK DENSITY IN UNSAT ZONE,$\left.g / C \mathbb{C O}^{*} 33\right)$

UNSATURATED ZONE THICKNESS

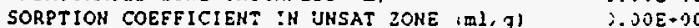

OPTIONAL LOSS RATE CONSTANT FOR SCURCE $\left., \ldots-1,0,30 \varepsilon_{*}\right)$

INITIAL MASS OR ACTIVITY (mg or $(\mathrm{I}) \quad$

MOLECULAR WEIGHT Ig/mOlO,

SOUUBILITY LIMIT (mg/L) $1.30 E .06$

HALF-LIFE(S) OF CONTAMINANT AND PROGENY (Y) $1.25 E+91$

BULK DENSITY OF AQU:FER $(\mathrm{g} / \mathrm{em} \cdots 3) \quad \therefore .00 E+D$

POROSITY OF AQUIFER

SORPTION COEFEICIENT(S) IN AOUIFER (mL/g)

DISPERSIVITY $X$ DIRECTION $\mathrm{m}$ ) :OOE+0

DISPERSIVITY $Y$ OIRECTION (D)

DISPERSIVITY $Z$ DIRECTION (a)

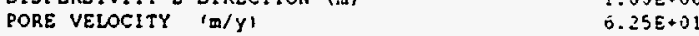

WELL SCREEH THICKMESS (m)

DISTANCE TO RECEPTOR ALONG X AXIS mI 1.05E+02

DISTANCE TO RECEPTOR ALCNG $!$ AXIS

DISTAHCE TO RECEPTCR ALONG 2 AX!5

RADIOLOGICAL DOSE CONVERSION FACTOR $r \mathrm{em} / \mathrm{C}: 1,3.20 E+23$

UNITS OF CONTAMINANT

LIMITING SOIL CONCENTRATIOU CALCULATION

$\gg \gg$ INITIAL ACTIVITY CONVERTED TO MASS mg) $7.25 E-02$ $\gg$ VALUES CALCULATED IN SOURCE SUBROUTINE

$5.8000 E+00$

RETARDATION EACTOR, SI (SATURATEDI

RETARDATION EACTOR ISI USATURATEDI

SOLWBILITY LIMITEO MASS $\mathrm{mgl}$

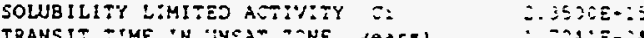

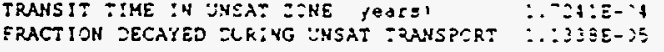

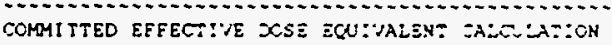

COMPOSITS DOSE CONVERS:CN FACTCR rem, $\tau:$ :

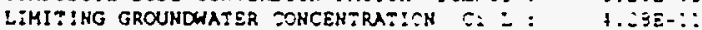

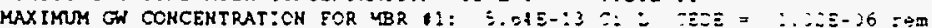

MAXIMM CEDE $1.32 \mathrm{E}-36=0 \mathrm{~m}$

PEAX TIME (Y):

LIMITIHG SOIL CONCEHTRATION 'C: II*+3,: :.4B:5-)6

LIMITING SOIL COKCENTRATION, $\left.\mathrm{C}_{1}, \mathrm{~kg}\right): \quad \vdots 55 \mathrm{JE}-\mathrm{p}$

LIMITING INVENTORY IN SOIL (CI::

LIMITING INVENTORY IN SOIL (mg): $\quad 5.497 E+30$

SPECIF:C ACTIVITY, C:/q1: $2.552 \mathrm{E}+32$

EXECLTICH T:ME iseconds)

TIME OF RUN :0:39:53.3

DATE OF RUN : $105,90^{\circ}$

$\gg>$ TILE OF PROSECO

C-11

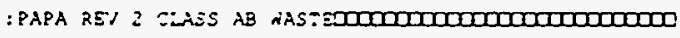

GAUSSIAM QUADRATURE SOWTION

MODEL OPTIONS

IMOOE : 1

KFLAG: 1 :O\}CONC YS TIME; I: MPEAK CONC AVD LIMIT:NG SOIL ECNC

IMODEL: 1 (!) SURF OR BLRIEE SOLRCE; , 2:PCND SOURCE: 3 :ABÜATES SOURCE FUNCTION

ITYPE: 0 (0) VERT AVG: (i) WON-VERT AVG: 3) AVG ERCM $0: 0 ~ Z O$

\\INPUT DATA

..........................................

D-3 


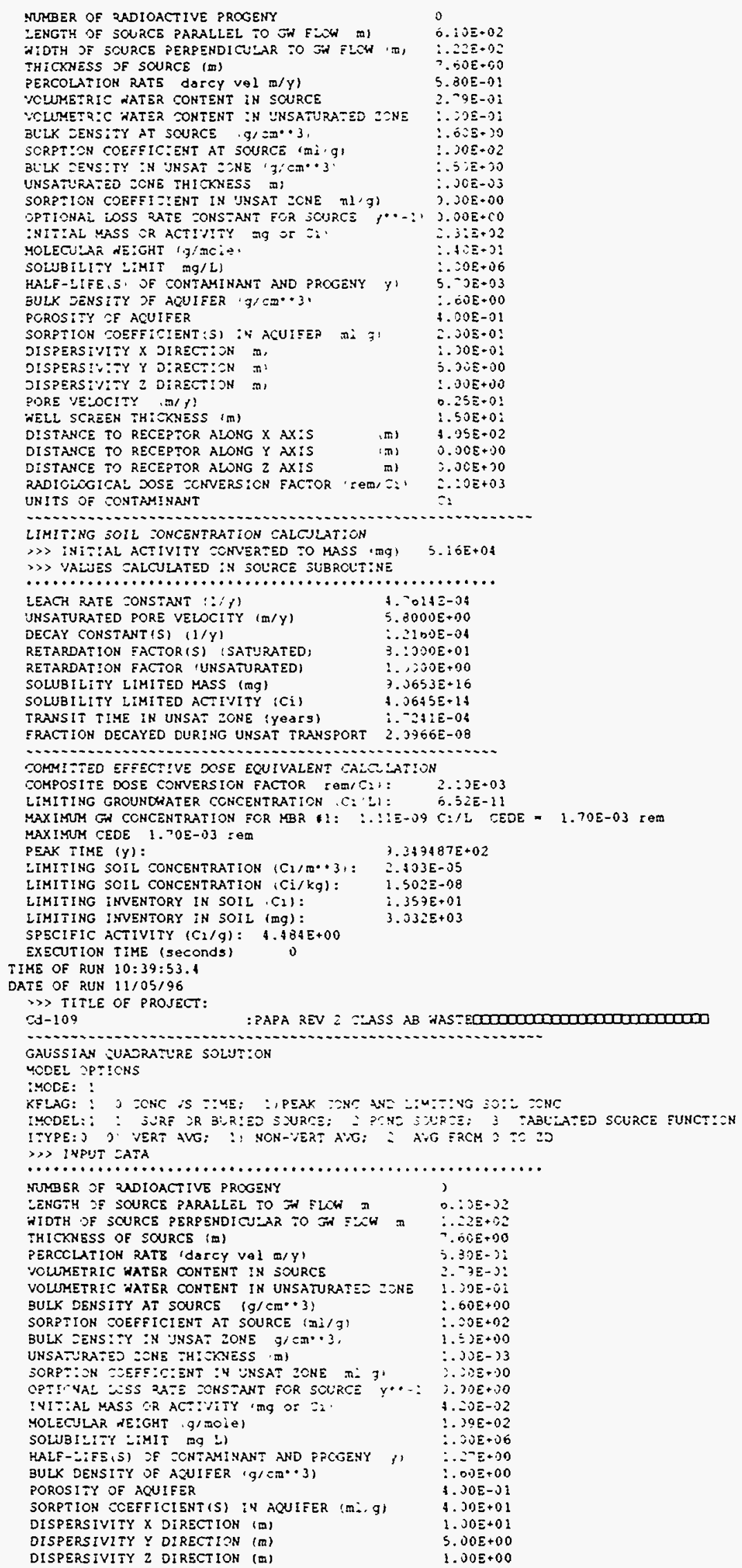




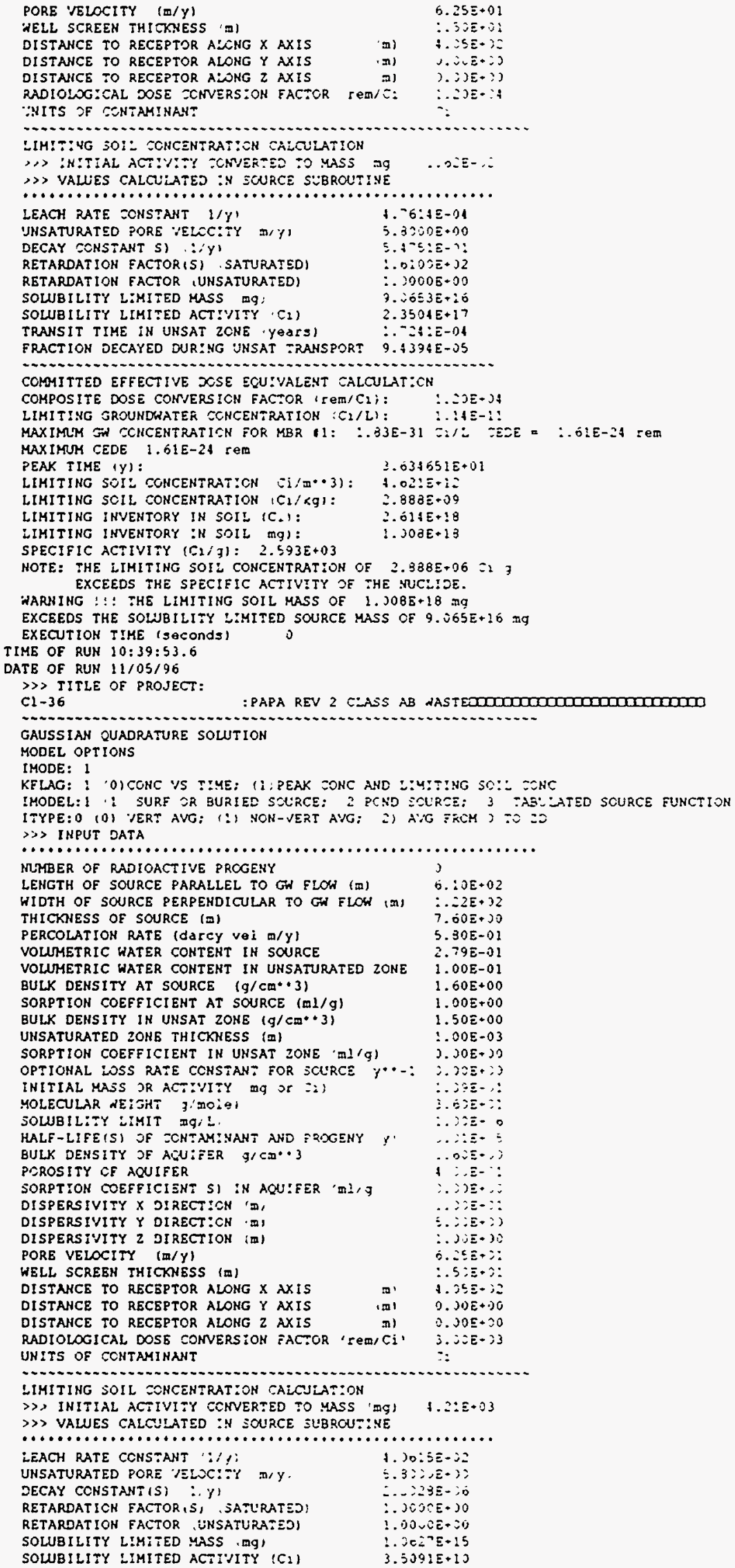




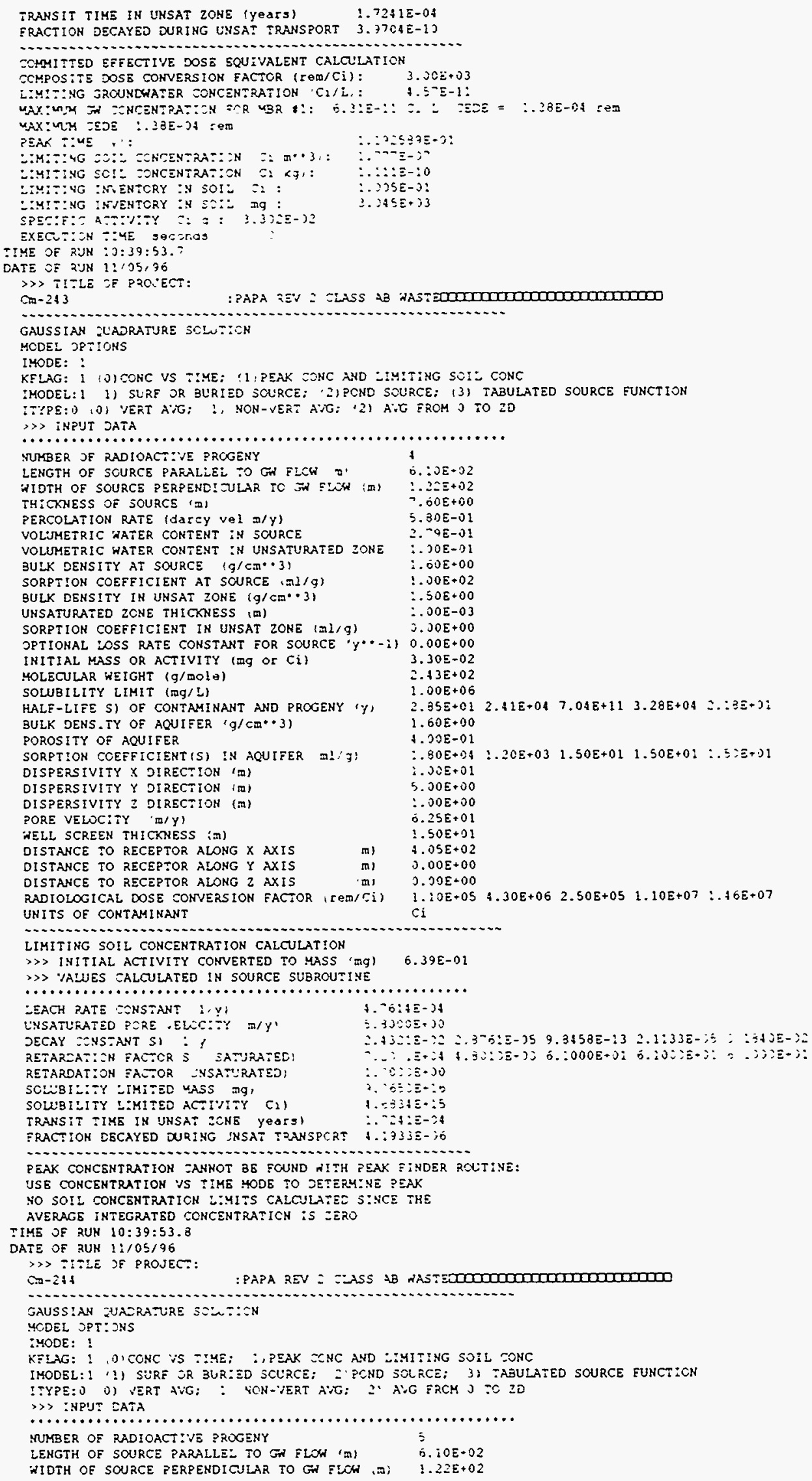




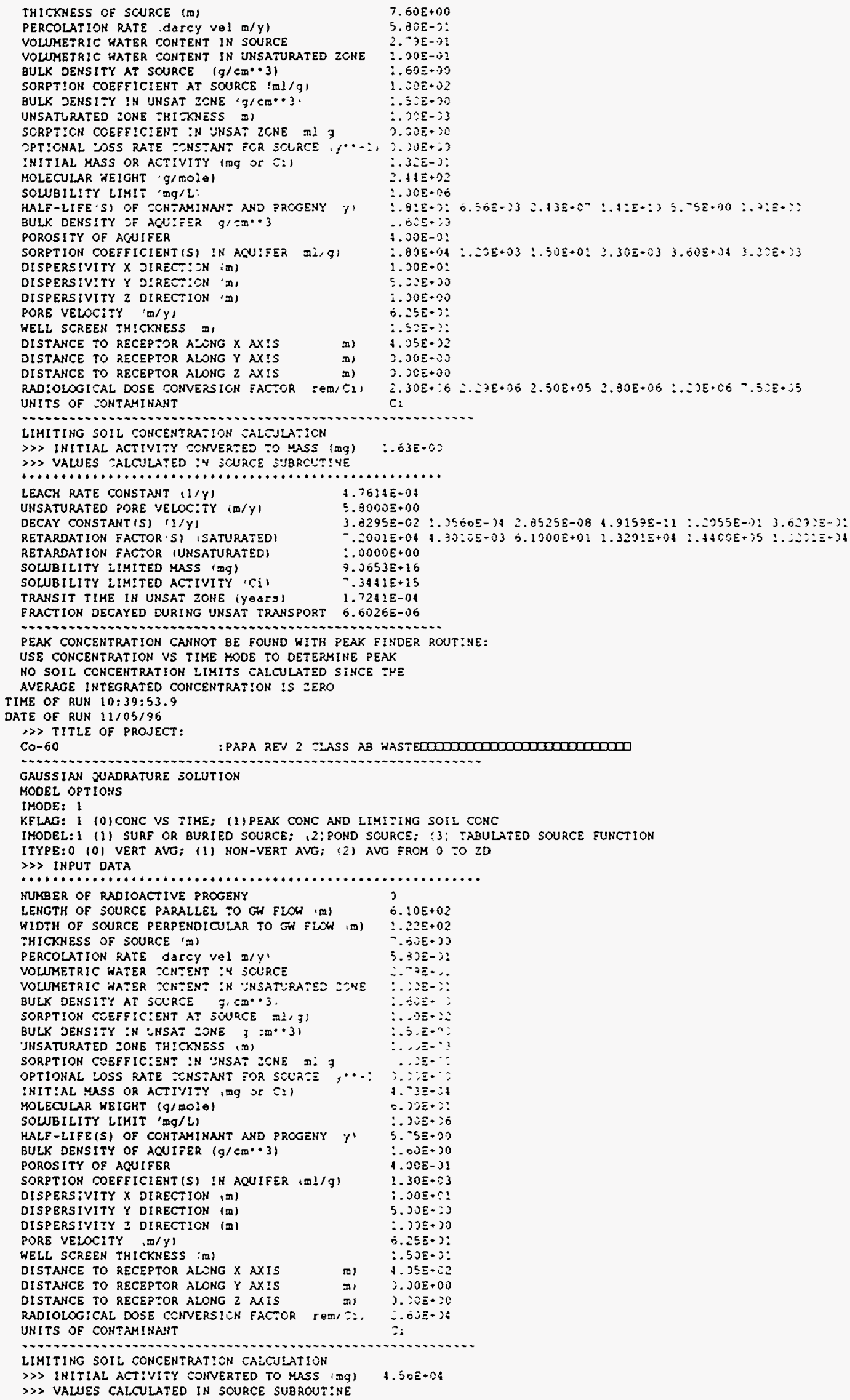




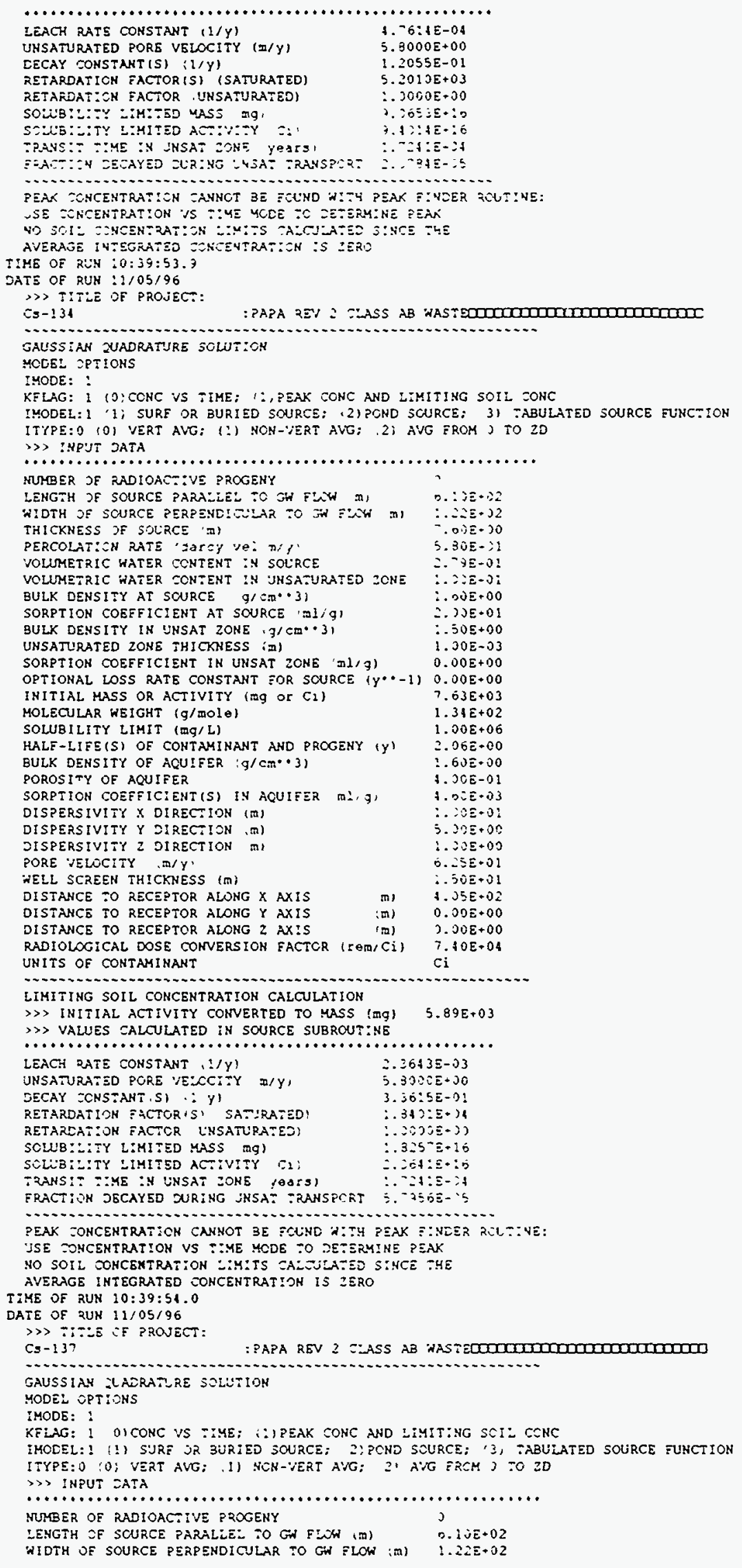




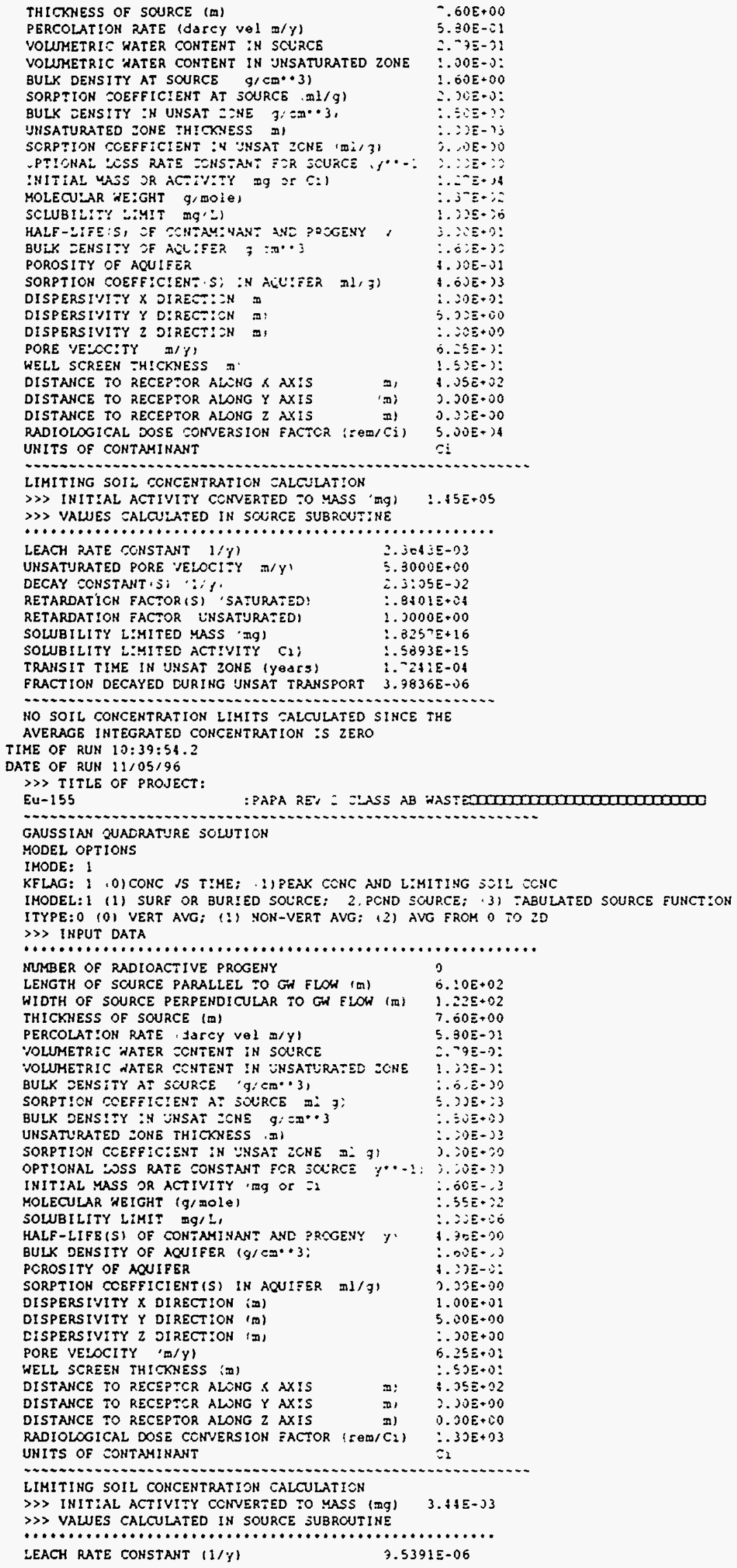




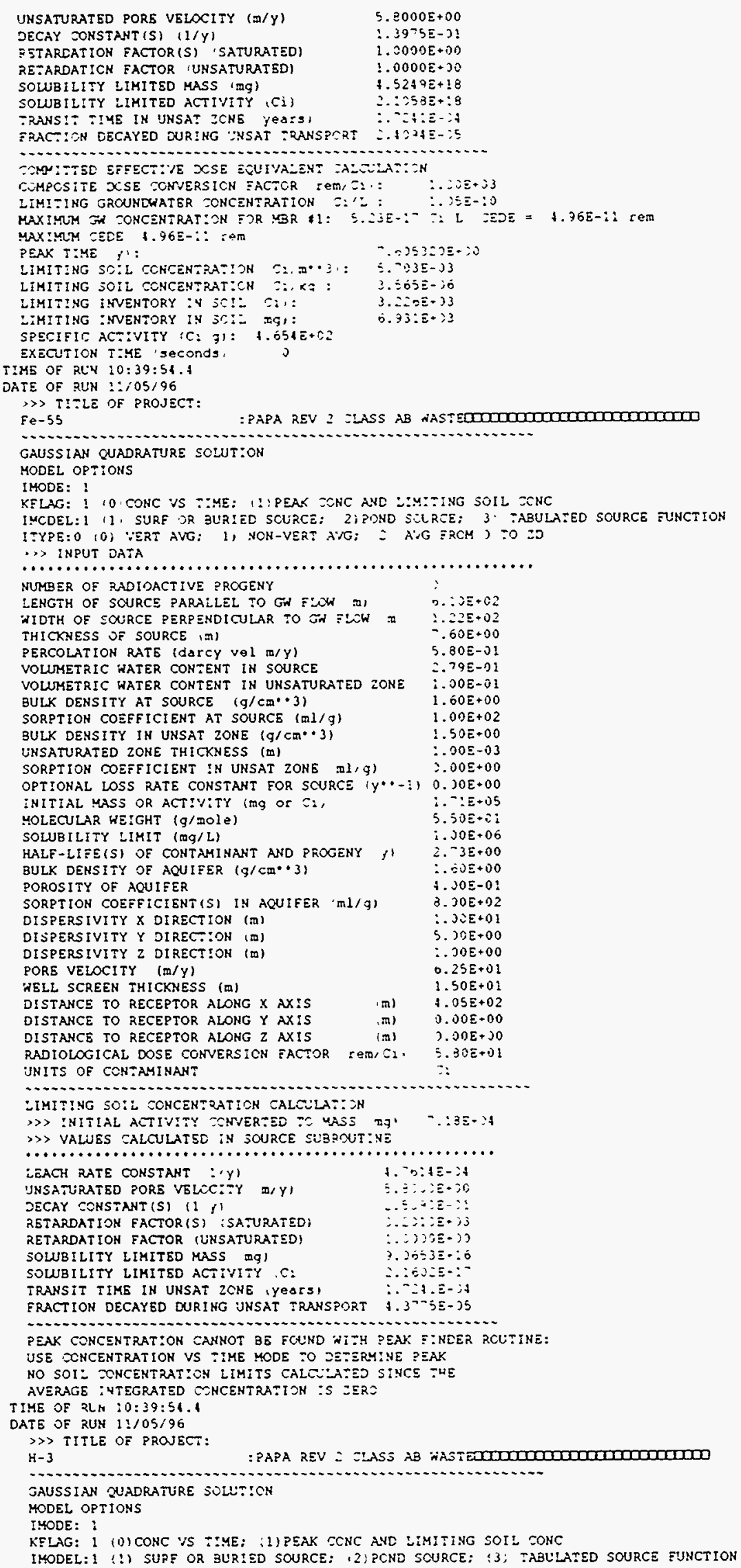


ITYPE:0 (0) VERT AVG; (1) NON-VERT AVG: (2) AVG ERCM 0 :O 20 $\gg$ INPUT DATA

\begin{tabular}{|c|c|}
\hline 3ER OF RADIOACTIVE PROGENY & 0 \\
\hline LENGTH OF SOURCE PARALEEL TO FN FLON (m) & $6 .: 0 E+02$ \\
\hline $\begin{array}{l}\text { WIDTH OF SOURCE PERPENDI UULAR TO FH FOON (m) } \\
\text { THICKNESS OF SOURCE }(\mathrm{m})\end{array}$ & $\begin{array}{l}1.22 E \cdot 02 \\
\because .0) E+20\end{array}$ \\
\hline PERCOLATION RATE dASCY Jel $\mathrm{m} / \mathrm{yl}$ & $5-j 3 E-3:$ \\
\hline VOLUMETRIC NATER ICNTENT IN SCURCE & $\therefore 99-\because:$ \\
\hline YOLLMETRIC WATER ZCNTËT :N SNSATLRATSE ZLNE & $\therefore: 2 \Xi-\grave{i}:$ \\
\hline BULK DENSITY AT SCURCE $(g /=8 \cdots 3)$ & $\therefore 60 \mathrm{E}+09$ \\
\hline SORPTICN COEFFICIENT AT SCURCE $\mathrm{m}: / \mathrm{gl}$ & $\therefore .0 \div E+3 j$ \\
\hline BULK DENS:TY IN UNSAT ZCNE $\quad \exists /=\pi \cdots 3$ ! & $: .50 E+20$ \\
\hline UNSATURATED ZONE :H: FNESS & $: .228-23$ \\
\hline JORFT:ON COEFF:Z:ENT :4 .SSAT 2ONE DI & $\therefore 20 \varepsilon-20$ \\
\hline GPTIONAL LOSS RATE ISNSTANT IOR SCLRCE,$\cdots+:$ & $3.20 \varepsilon+00$ \\
\hline IHITIAL MASS OR ACTIV:TY mg or $Z:$ & $3.3 j E+05$ \\
\hline MOLECLAR NEIGHT (q/MOLE) & $3.30 E+30$ \\
\hline SOLUBILIT! LIM!T SgIL, & $\therefore \supset 0 \bar{E}+1) 6$ \\
\hline HALF-LIFE(S) SF TCNTAKENANT AND ?ROGENY & $\therefore .23 E+1 \mathrm{i}$ \\
\hline BULK DENSITY SF ACLIFER $\quad$ / $/ \mathrm{O}+3$ & $\therefore 60 E \cdot 30$ \\
\hline POROSITY OF ACUIFER & $4.2(5-3:$ \\
\hline SORPTION COEFFIC:ENT'SI IN ACUIFER & $0.20 E-100$ \\
\hline DISPERSIVITY X DIRECTICN (回) & $1.20 E+01$ \\
\hline DISPERSIVITY Y DIRECTION (D) & $5.30 E+00$ \\
\hline DISPERSIVITY $Z$ DIRECIION (m) & $1.00 E+00$ \\
\hline PORE VELOCITY $(\mathrm{m} / Y)^{\prime}$ & $6.25 E+01$ \\
\hline WELL SCREEN THICKNESS $(\mathrm{m})$ & $2.50 E+01$ \\
\hline DISTAHCE TO RECEPTCR ALONG $X$ AXIS & $4.055+02$ \\
\hline DISTANCE TO RECEPTOR A:CHG Y AXIS & $3.305+00$ \\
\hline DISTANCE TO RECEPTOR ALONG 2 AX:S & $2.005 \cdot 30$ \\
\hline RADIOLOGICAL DOSE CONVERS:ON FACTOR & $0.30 E+32$ \\
\hline UNITS OF ZONTAMINANT & $=2$ \\
\hline
\end{tabular}

LIMITING SOIL GCNCENTRAT:ON IALCUMAT:ON

$\Longrightarrow$ INIT:AL ACTIYITY ZCNVERTED TO YASS IgI $3.04 E+04$ $\gg$ VALUES CALCILATEO IN SOLACE SUBRCUT:NE

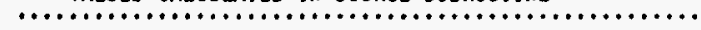

LEACH RATE CCNSTANT (l'Y) $=-353 E-0]$

UHSATURATED PORE VELCITY (TO/Y)

DECAY CONSTANT (S) $(1 / Y)$ 5.6353E-02

RETARDATION FACTOR(S) (SATURATED) $1.0000 E+00$

RETARDATION EACTOR .UNSATURATEDI $1.0000 E+00$

SOWBILITY LIMITEO MASS (mg) $1.5780 E+14$

SOUBILITY LIMITED ACTIVITY (Ci) $1.5301 \mathrm{E}+15$

TRANSIT TIME IN UNSAT ZONE (years) $1.7241 \mathrm{E}-34$

FRACTIOH DECAYED DURING UNSAT TRANSPORT $9.7161 \mathrm{E}-196$

COMAITTED EFFECTIVE DOSE gQUIVALENT JALCU:ATION

COMPOSITE DOSE CONVERSION FACTOR (rөm/Ci): 6.30E+01

LIMITING GROUNDAATER CONCENTRATION, Ci/Ll: $2.27 \mathrm{E}-09$

MAXIMUM GH CONCENTRATION FOR MBR 11: 2.97E-04 $C_{1} / L$ CEDE - :.37E+01 rem

MAXIMIM CEDE $1.37 E+01$ rem

PEAK TIME $(Y)$ :

PEAK TIME (Y):

$\quad 4.572 \mathrm{E}-06$

LIMITING SOIL CONCENTRATICN (Ci/kg): $\quad 2.858 \mathrm{E}-09$

LIMITING INVENTORY IN SOIL :Ci,: $\quad 2.586 E+00$

LIMITING INVENTORY IN SOIL (mg): $2.667 E-01$

SPECIFIC ACTIVITY $\left(C_{2} / g\right): 9.690 \mathrm{E}+03$

EXECUTION TIME IsecondsI 0

TIME OF RUN 10:39:51.5

DATE OF RUN $11 ; 05 / 96$

$\gg$ TITLE OF PROISC:

$1-i 29$

:PAPA REV 2 T:ASS AB AASTEDOW0000010001000000

10........

GAUSSIAH ZUADRATJRE SOLUT:OH

MODEL OPTIONS

IMODE: 1

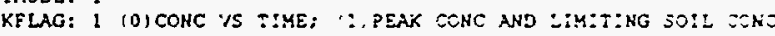

IHODEL: 1 (1) SURF ZR SURIED SCURCE: 2:PCHD SCLRCE: I TABULAEDD SCURCE SUHCT:ON

IEYPE:0 O) VERT AVG; (! YCN-VERT AVG; Z) AVG ERCM O :O

$\gg$ INPUT DATA

NUMBER OF RADIOACT:US PRCGEHY

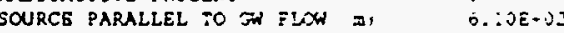

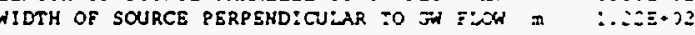

THICKNESS OF SQURCE (m)

PERCOLATION RATE (darey vel $\mathrm{m} / \mathrm{y}$ ) $5.30 \overline{\mathrm{e}}-\mathrm{jl}$

VOLUMETRIC WATER CONTEHT IN SCURCE

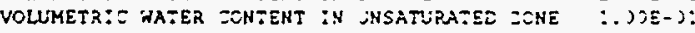

BULK DERS:TY AT S YURCE $g /=\pi \cdots 31$

SORPTIOY TCEFFIC:ENT AT SCLRCE II, 31

AT SCLRE

$\therefore .5,3 E+30$

UNSATJRATED ZCNE Th. SNESS MI

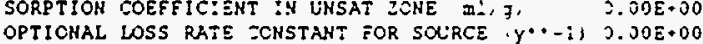

INITIAL MASS OR ACTIVITY img OR $\left.C_{2}\right)$,

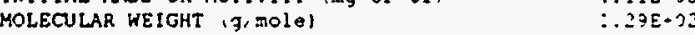

SOWBILITY LIKIT , Jg/L)

HALF-LIFE(S) OF CONTAMINANT AND PROGENY (Y) $1.57=+07$

BULK DENSITY OF ACU!FER $(\mathrm{g} / \mathrm{Cm} \cdots 3) \quad$ :. $30 E+00$

POROSITY OF AOUIFER

POROSITY OF AQUIFER
SORPTION COEFFICIENT(S) IN AQUIFER (ml/g) 


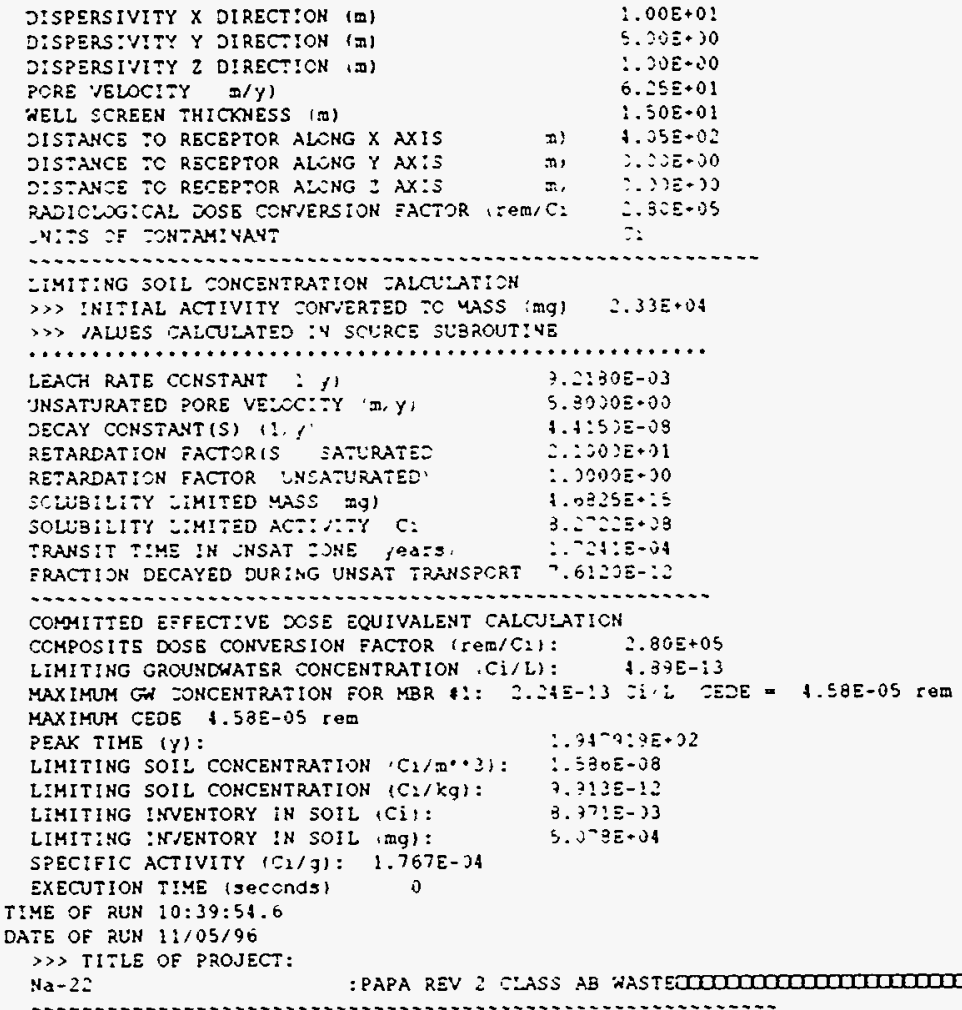




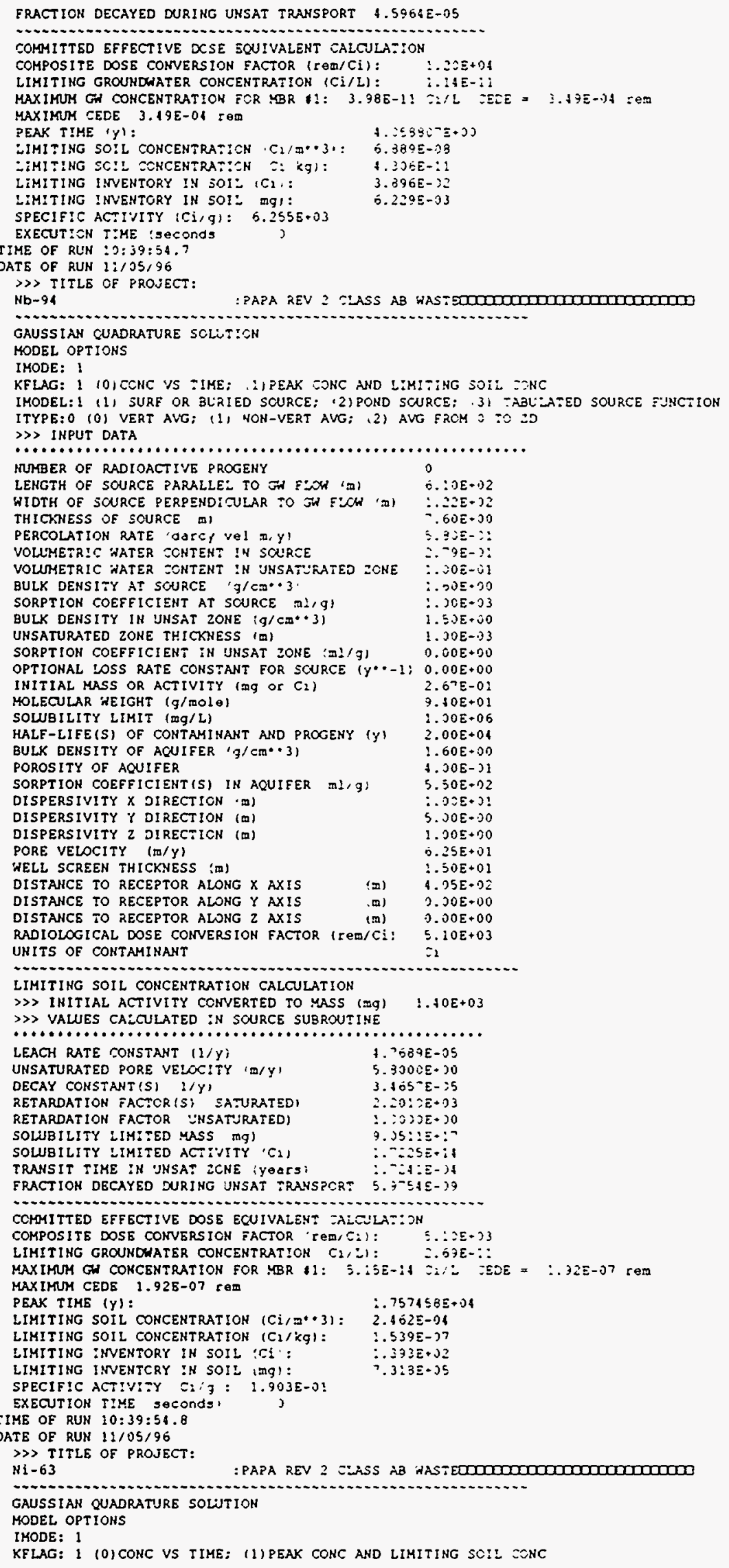


IHODEL: 1 ( $)$ SURE OR BURIED SOURCE; (2) POND SOURCE; 13; TABULATED SOURCE EUNCTION ITYPE:0 i0 VERT AVG: (1) NON-VERT AVG; .2: AVG FRCM $\%$ TO 20

$\because$ :NPLT SATA

\begin{tabular}{|c|c|}
\hline NURBER OF RADIOACTIVE PROGENY & \\
\hline EENGTH OF SCLRCE PARALLEL :O GN FLW $\mathrm{ml}$ & 6.:JE-G2 \\
\hline 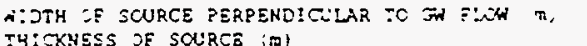 & $\begin{array}{l}\therefore: \pm E+32 \\
-.00 E+30\end{array}$ \\
\hline ERCOLATION RATS darCY vel w/YI & $5.30 \mathrm{E}-31$ \\
\hline HOLMETA:Z NATER SONTENT :N SUURCE & $\therefore-35-9:$ \\
\hline SCLUMETRI G WTER CONTSNT ON UNSATURATED ZCNE & $\therefore 30 \mathrm{~B}-31$ \\
\hline ULK DENSITY AT SCURCE $\left(g / \mathrm{CM}^{\prime} \cdot 3\right)$ & $\therefore .00$ \\
\hline SRPT:ON CCEEFICIENT AT SCURCE (ML/A) & $\therefore 30 \Xi+02$ \\
\hline 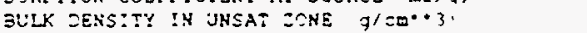 & $\therefore \quad 53 \pm+20$ \\
\hline UNSATURAIED ZONE -H:XNESS & $\therefore . \partial 0 E-33$ \\
\hline SORPTION COEFFICIENT IN UNSAT ZONE w2, J' & $3.30 E+00$ \\
\hline OPTICNAL WOSS RATE CONSTANT FOR SZUREE,$\cdots-!$ & $3.30 E-09$ \\
\hline INITEAL YASS OR ACTIVITY Tg Or $Z 1$, & $5.33 \approx+01$ \\
\hline MCEECULAR WEIGHT g/mOLe) & $3.30 \mathrm{E}+22$ \\
\hline SOUUBILITY :IMIT $(m g / L)$ & $\therefore .30 \varepsilon+06$ \\
\hline HALF-LISEIS: CE TONTAMINANT ANO PROGENY & $\therefore 30 \equiv-02$ \\
\hline 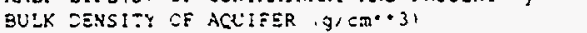 & $\therefore O \partial E+\supset 0$ \\
\hline OSITY OE AQUSEER & $+.30 E-01$ \\
\hline SORPTION COEFFICIENE (S) IN AGUIFER & \\
\hline DISPERSIVITY X OIRECT:ON $(\mathrm{m})$ & $\therefore .30 \Omega+01$ \\
\hline DISPERSIVITY Y DIRECT:ON $(\mathrm{m})$ & $5.20 \varepsilon+00$ \\
\hline DISPERSTVITY 2 SIRECT:ON $(2)$ & \\
\hline PORE VELOCITY $(\square / Y)$ & $0.25 E+01$ \\
\hline WELL SCREEN THICKNESS (m) & $\therefore .50 E+01$ \\
\hline DISTANCE TO RECEPTOR ALNG $X$ AXIS & 4. $35 E+02$ \\
\hline ISTANCE TO RECEPTOR ALONG Y AXIS & 2.209 \\
\hline DISTANCE TO RECEPTOR ALCNG $Z$ AXES & ). $20 E+20$ \\
\hline $\begin{array}{l}\text { RADIOLOGICAL DOSE CCNVERICN FACTOR }\left(\mathrm{rem} / C_{2}\right) \\
\text { UNITS OF CONTAMINANT }\end{array}$ & $\begin{array}{l}\vdots .+0 E-01 \\
\vdots 2\end{array}$ \\
\hline
\end{tabular}

LIMITING SO:L CONCENTRATION CALCULATION

$\Longrightarrow$ INITIAL ACTIVITY CONVERTED TO MASS mq $3.368+02$

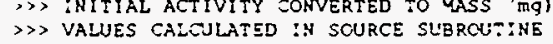

..............................

UNSATURATED PORE VELOCITY $(a / Y)$

DECAY CONSTANT (S) $(1 / Y)$

RETARDATION FACTOR(S) SATLRATEDI $\quad: 20: 2 E-03$

SOLUBILIT: TYITEO MASS (NG)

SOLUB:T-

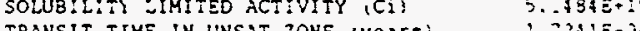

FRACTION DECAYED DURING UNSAT TRANSPORT $\quad:, 2,: 35:=36$

COMIITED EFFECTIVE DOSE EQUIVALENT CALCNAATION

TCMPOSITE DOSE CONVERSION FACTOR ( $\left.\mathrm{rem} / \mathrm{C}_{2}\right)$ : $5.40 E+0$

LIMITING GRUUNDWATER CONCENTRATION $(\mathrm{CI} / \mathrm{L})$ : $2.5+\mathrm{E}-0$

MAXIMUM GN CONCENTRATION FOR MBR $11: 4.48 E-16 \mathrm{CI} / \mathrm{L}$ CEDE $=1.77 \mathrm{E}-11$ rem

MAXIMUM CEOE 1.775-11 TED

PEAK TIME (Y):

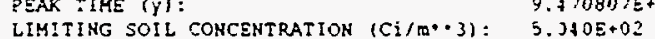

$\begin{array}{ll}\text { LIMITING SOIL CONCENTRATION }\left(\mathrm{Ci} / \mathrm{m}^{*} \cdot 3\right): & 5.3405+02 \\ \text { LIMITING SO:L CONCENTRATION }\left(\mathrm{C}_{1} / \mathrm{kg}\right): & 3.50 \mathrm{l}-0 \mathrm{i}\end{array}$

$\begin{array}{ll}\text { LIMITING SO:L CONCENTRATION }\left(\mathrm{C}_{2} / \mathrm{kg}\right): & 3.250 \mathrm{E}-3 \mathrm{i} \\ \text { LIMITING INVENTORY IN SOIL }(\mathrm{Ci}): & 2.851 \mathrm{l}+08\end{array}$

$\begin{array}{ll}\text { LIMITING INVENTORY IN SOIL }(\mathrm{C} i): & 2.351 E+08 \\ \text { LIMITING INVENTORY IN SOIL }(\mathrm{mg}): & 5.220 \Sigma+09\end{array}$

SPECIEIC ACTIVITY $(2 \mathrm{i} / 9): \quad 5.679 \mathrm{9}+01$

EXECUTION TIME iseconds) 0

TIME OF RUN 10:37:55.

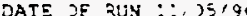

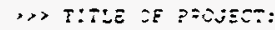

$4:-53$

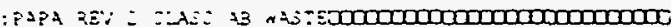

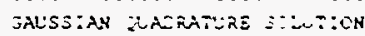

HCDE: ZPT:ZNS

YCLE:

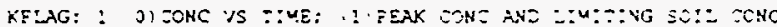

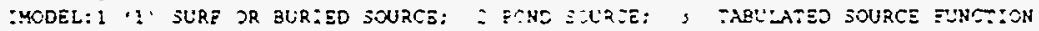

ITYPE:0 OI VERT AVG; :: YON-VERT AVG: : A'G ERCM J TO 20

$$
\rightarrow>\text { ZNPUT EATA }
$$

MURBER OF RAOIOACT:VE ?ROGEHY

LENGTH OF SOURCS PARALLEL TO

WIDTH OF SOURCE PERPENDICULAR TO FW ELOK mI

THICKNESS OF SOURCE (B)

PERCOLAT:CN VATE darCY Vel M/Y

VOUMETR: W WATER TONTSNT IN SUURCE

VCZMETRIC RATER EONTSNT ON UNSATURATED ZONE

BL:K ZENS:TY AT ECLRZE

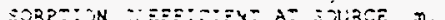

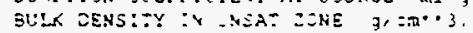

INSATURATES ZONE T+TKNESS MI

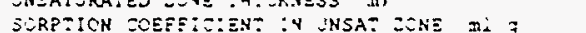

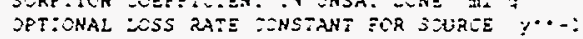

INIT:AL MASS JR ACTIY:TY 'mg Or $:=1$

MOLECULAR HE:OHT g/MOle

SOLUBILITY LIMIT :OG/I

HALF-LLEE(S) OE CONTAMINANT AND PROGENY (Y1

BULK DENSITY OF AQUIEER $(\mathrm{g} / \mathrm{cm} \cdots 3)$

POROSITY OE ACUIFER
5. $: 2 E+02$

$\therefore .22 \Xi+02$

$7.60 E+00$

$5.305-2:$

$\because-\because 2 z-3:$

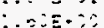

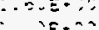

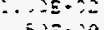

$\therefore: 3 E-j 3$

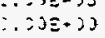

$\therefore 305+20$

$\vdots .33 \varepsilon-01$

$5.33 \mathrm{E} \cdot 0:$

1. $30 E+06$

$-.3 \mathrm{CE}+04$

$\therefore-00 E+00$ 


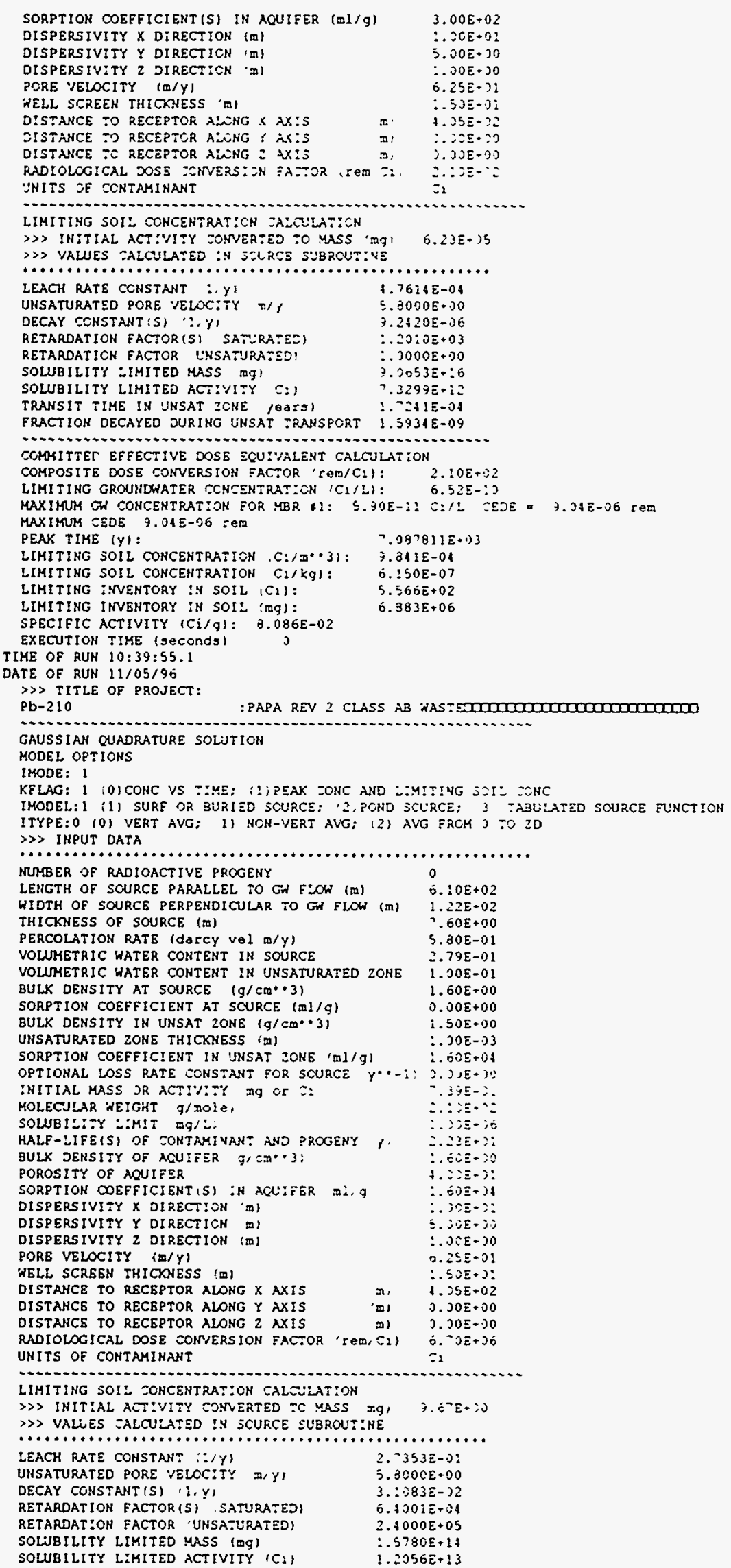




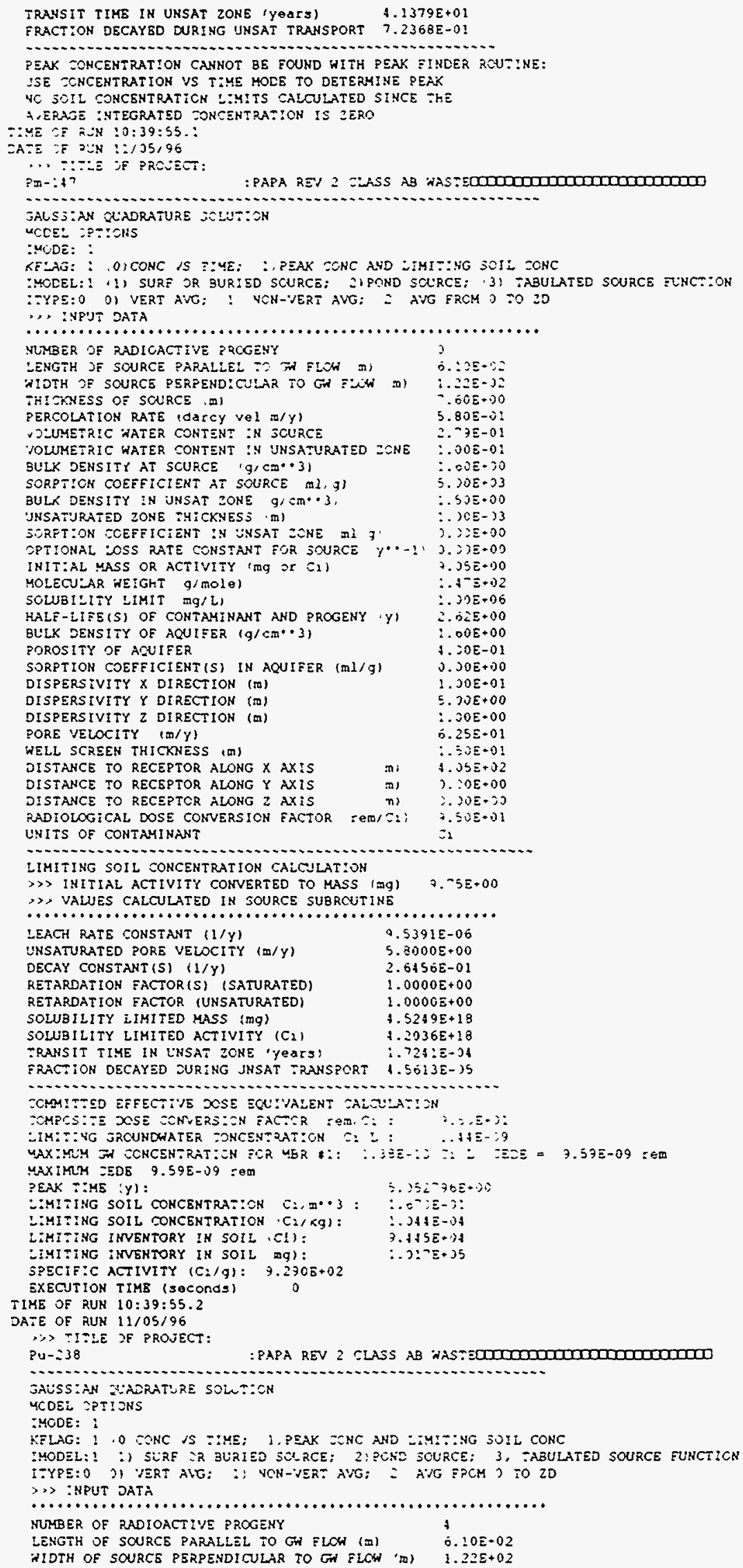




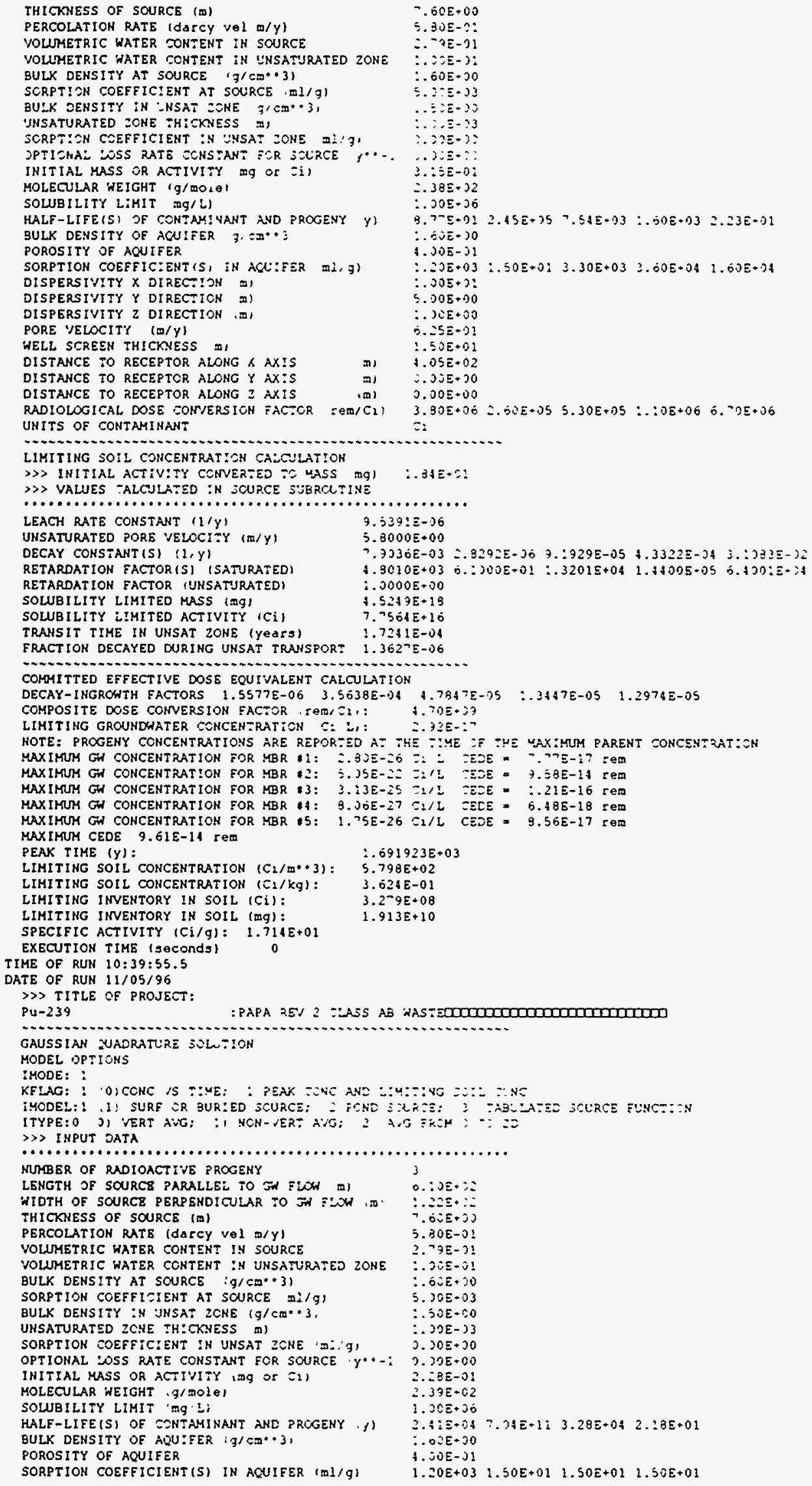




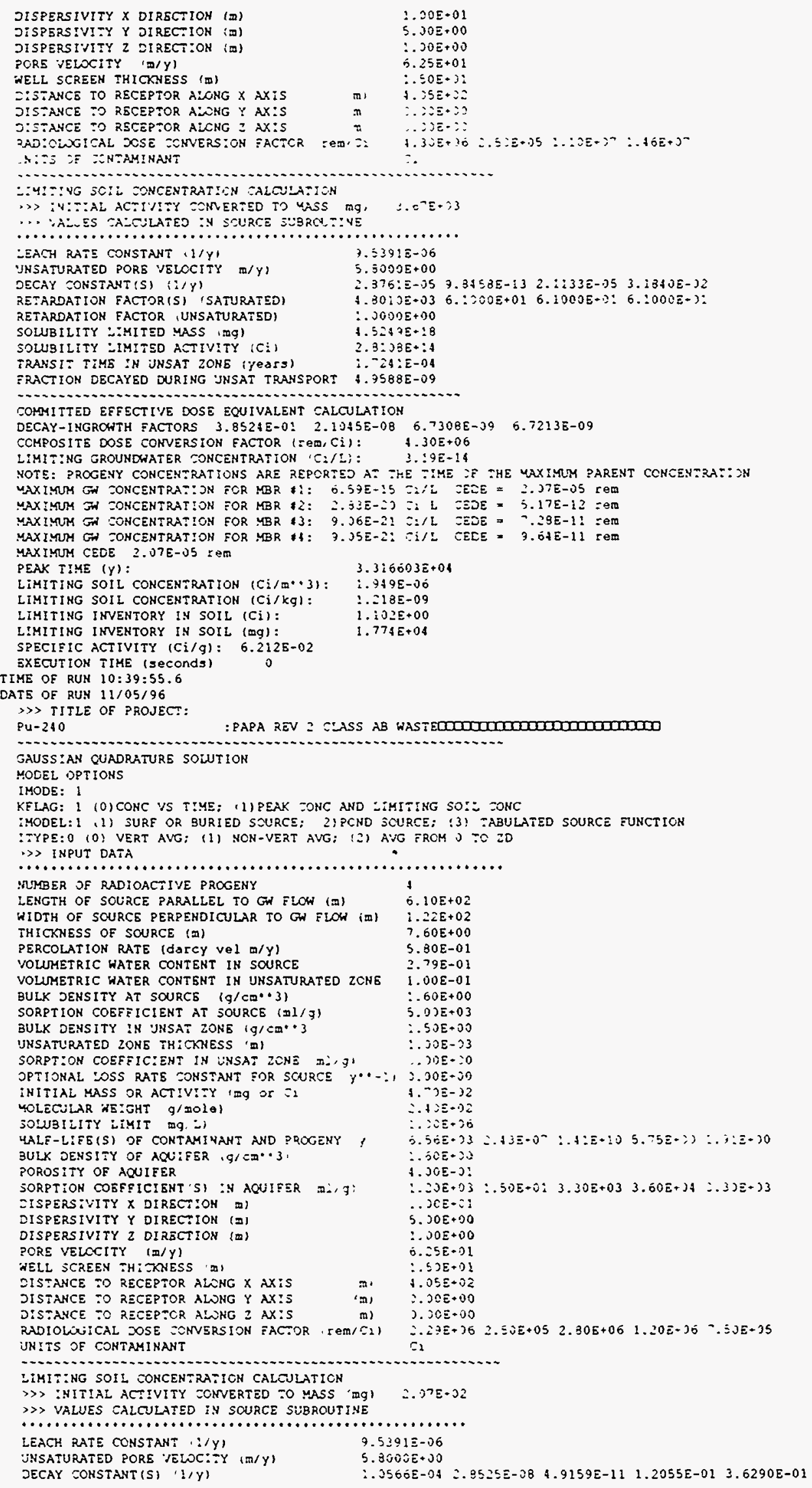




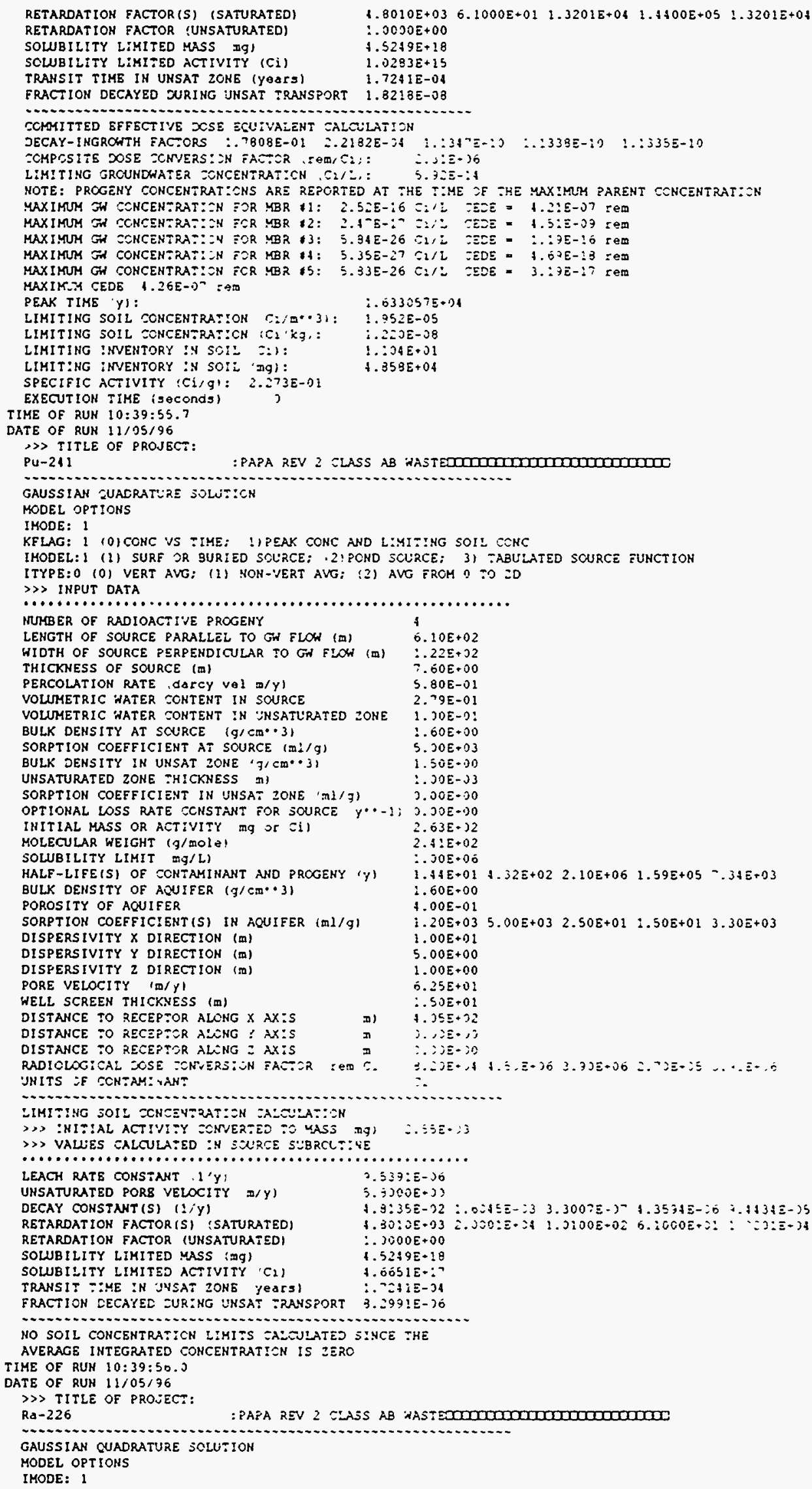


KFLAG: 1 (O)CONC VS TIME; (1)PEAK CONC AND LIMITING SOIL CONC IMODEL: 1 (1) SURF OR BURIED SQURCE; (2) POND SCURCE; (3) TABULATED SOURCE FUNCTION :IYPE:0 (0) VERT AVG: (1) NON-VERT AVG; (2) AVG FROM 0 IO ZD

\> INPUT DATA

\begin{tabular}{|c|c|}
\hline RBER OF RADIOACTIVE PROGEMY & 1 \\
\hline ENGTH OF SOURCE PARALLEL TO GW FLOW & 5.:2E+02 \\
\hline AIDTH OF SOURCE PSRPENDICULAR TO FH E:LW & $\because .225+02$ \\
\hline $\begin{array}{l}\text { THICKNESS OF SOURCE }(m) \\
\text { PERCOLAT:SN RATE darCy vel m,y\} }\end{array}$ & $.605+00$ \\
\hline $\begin{array}{l}\text { PERCOLAT:OA RATE dOTCY VEL DYY } \\
\text { VOLUMETRIC HATER DONTSHT IN SCURCE }\end{array}$ & 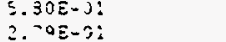 \\
\hline VOLUMETRIC HATSR CONTENT IV UNSATURATED IONE & $\therefore 205-01$ \\
\hline BULK JENSITY AT SCURCE (q, (m) $\cdots 3)$ & $\therefore .00 \Xi+90$ \\
\hline SRPTION COEFEIC:ENT AT SOLRCE DL/G) & $\therefore 3 \varepsilon \mathrm{E}+02$ \\
\hline JLK DENSITY :N ONSAT ZSNE $\quad 3=\pi \cdots 3$ & $\therefore \equiv 0 \Omega+20$ \\
\hline JNSATURATED ZONE THICWNESS (m) & $\therefore .005-03$ \\
\hline SORPTION COEFEICIENT IN UNSA: ZONE ME, gl & $3.00 \mathrm{E}+04$ \\
\hline PTIONAL LOSS RATE CONSTANT SOR SCLRCE YY $\cdots-$ : & $0.005+00$ \\
\hline INITIAL MASS OR ACTIVITY img or $=: 1$ & $.405-03$ \\
\hline MOLECULAR WESGHT (g/TOLO) & $2.25 \Sigma+02$ \\
\hline OWBILITY IIMIT (mg/L) & $1.005+06$ \\
\hline LF-EIEE (S) OF CONTAMENANT AND PRDGENY ( $i$ ) & $\therefore .60 E+03 \quad 2.23 E+01$ \\
\hline BULK DENSITY OF AQUIFER $\left(g / \mathrm{cs}^{*} 3\right)$ & $1.60 E+00$ \\
\hline DROSITY OF AQUIFER & 4. $00 E-01$ \\
\hline RPTION COEFEICIENT(S) IN AQUIFER & $3.60 \mathrm{E}+04 \quad 1.60 \mathrm{E}+04$ \\
\hline OISPERSIVITY $X$ DIRECTION ( $(0)$ & $1.00 E+01$ \\
\hline ISPERSIVITY Y DIRECT:ON (m) & $5.00 E+00$ \\
\hline ISEERS:VITY Z DIRECTION (D) & $1.20 \equiv=00$ \\
\hline PORE JELOCITY \{́⿴囗Y' & $0.25 E+0 !$ \\
\hline ELL SCREEN THICKNESS (m) & $\therefore 50 \Sigma+1) !$ \\
\hline ISTAMCE TO RECEPTOR ALONG $X$ AXIS & $4.255+32$ \\
\hline DISTANCE TO RECEPTOR ALUNG Y AXIS & $3.205-00$ \\
\hline DISTANCE TO RECEPTOR ALLNG I AXIS & $3.30 E \cdot 00$ \\
\hline $\begin{array}{l}\text { PADIOLOGICAL DOSE CONVERSION FACTCR , EEMSC:, } \\
\text { JNITS OF CONTMMINANT }\end{array}$ & $\begin{array}{l}1 .: 3 E+06 \quad 6 .-0 E+06 \\
\sigma_{2}\end{array}$ \\
\hline
\end{tabular}

JNITS JF CONTAMINANT

WMIT:NG SOIL CONCENTRATION CALCULATION

$\gg$ INITIAL ACTIVITY CONVERTED TO MASS $\mathrm{mgl}-.+8 \varepsilon+00$

$\rightarrow>$ VALUES CALULATED IN SOURCE SUBRCUT:ME

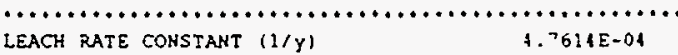

UNSATURATED PORE VELOCITY $(\mathrm{m} / \mathrm{Y}) \quad 5.3000 E+00$

.. $3322 E-04 \quad 3.1083 E-02$

RETARDATION EACTOR(S) (SATURATED) $\quad$ i. $1400 E+056.10018+04$

RETARDATION FACTOR (UNSATURATED) $\quad$..9000E+05

SOLUBILITY LIMITED MASS $(\mathrm{mg}) \quad 7,3653 E+16$

SOLUILITY LIMITED ACTIVITY (CI)

TRANSIT TIME IN UNSAT ZONE (YearS) $3.3: 04 E+01$

FRACTIOA DECAYED DURING UNSAT TRANSPORT 3.7531E-02

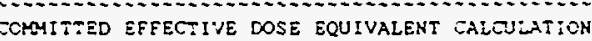

DECAY-I.HGRCHTH EACTORS T.2306E-08 $7.33: 8 E-08$

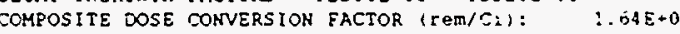

LIMITING GROUHDWATER CONCENTRATION (C:/L): $3.36 \mathrm{E}-15$

NOTE: PROGENY CONCENTRATIONS ARE REPORTED AT THE T:ME OF THE MAXIMUM PARENT CONCENTRATION

MAXIMUM GN CONCENTRATION FOR MBR $: 2: 3.73 E-30$ GLL CEDE $=7.36 E-21$ rem

MAXIMM GR CONCENTRATION FOR MBR 2: 2.01E-29 CI'L CEDE = 9.81E-20 TOM

MAXIMUM CEDE $1.05 E-19$ TOD

LIMITING SOLL CONCENTRATION $\left(\mathrm{C}_{2} / \mathrm{m} \cdot+3\right)$ : $1.245 E+07$

LIMITING SOIL CONCENTRATION $\left(\mathrm{C}_{2} / \mathrm{Xg}\right): \quad 7.778 E+03$

LIMITING INVENTORY IN SOIL (Ci):

LIMIEING INVENTCRY IN SOIL $\mathrm{mg}$ : : -.:LE+is

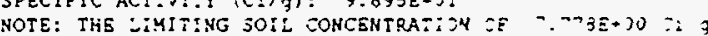

$$
\text { EXEEESS THE SPECIFIC ACTIVITY OF THE TUC:OSE. }
$$

EXEOUT:- Th THE saconds.

TIME OF RUN :3:39:56.3

CATS OF RUN $11 / 05 / 96$

\> T:T:E JF PRQUECT:

$\mathrm{Sb}-125$

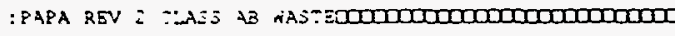

GAUSS:AN WJADRATURE SOLT:ON

MODEL OPTIONS

:MOOE: 1

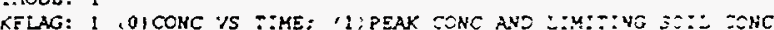

IMODEL: 1 II SURF OR SURIEO SOURCE: ZOND SOLKCE; 3 : ABULATEO SOURCE EUNCE:ON

ITYPE:0 (0) VERT AVG: (1) NON-VERT AVG: 2; A'O ERCM 0 TO 20

$\gg$ INPUT DATA

NUMBER OF RACICACT:VE PROGENY

LENGTH OF SCURCE PARALLE: TO FN FLW TI

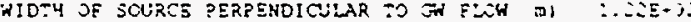

THICNSSS OF SOURCS

PERCOLATION RATE (dATCY Vel m/Y)

2.79E-01

VOUMETRIC HATER CCNTENT IN JYSATURATED ZONE $: J \geqslant E-0 !$

BULK DENSITY AT SOURCE (G/CM*3)

SORPTION COEEEICIENT AT SOURCE (MI/g)

BULK DENSITY IN UNSAT ZONE $\left(\mathrm{g} /=\mathrm{m}^{*} \cdot 3\right) \quad 1.50 \mathrm{~g}+00$

UNSATLRATED ZONE THTCKNESS (a)

SORPTION COEFEICIENT IN UNSAT ZONE (m1/g) $0.00 E+00$

ORTIONAL LOSS RATE CONSTANT FOR SOURCE $\left(y^{*}+1\right) 0.00 E+00$ 


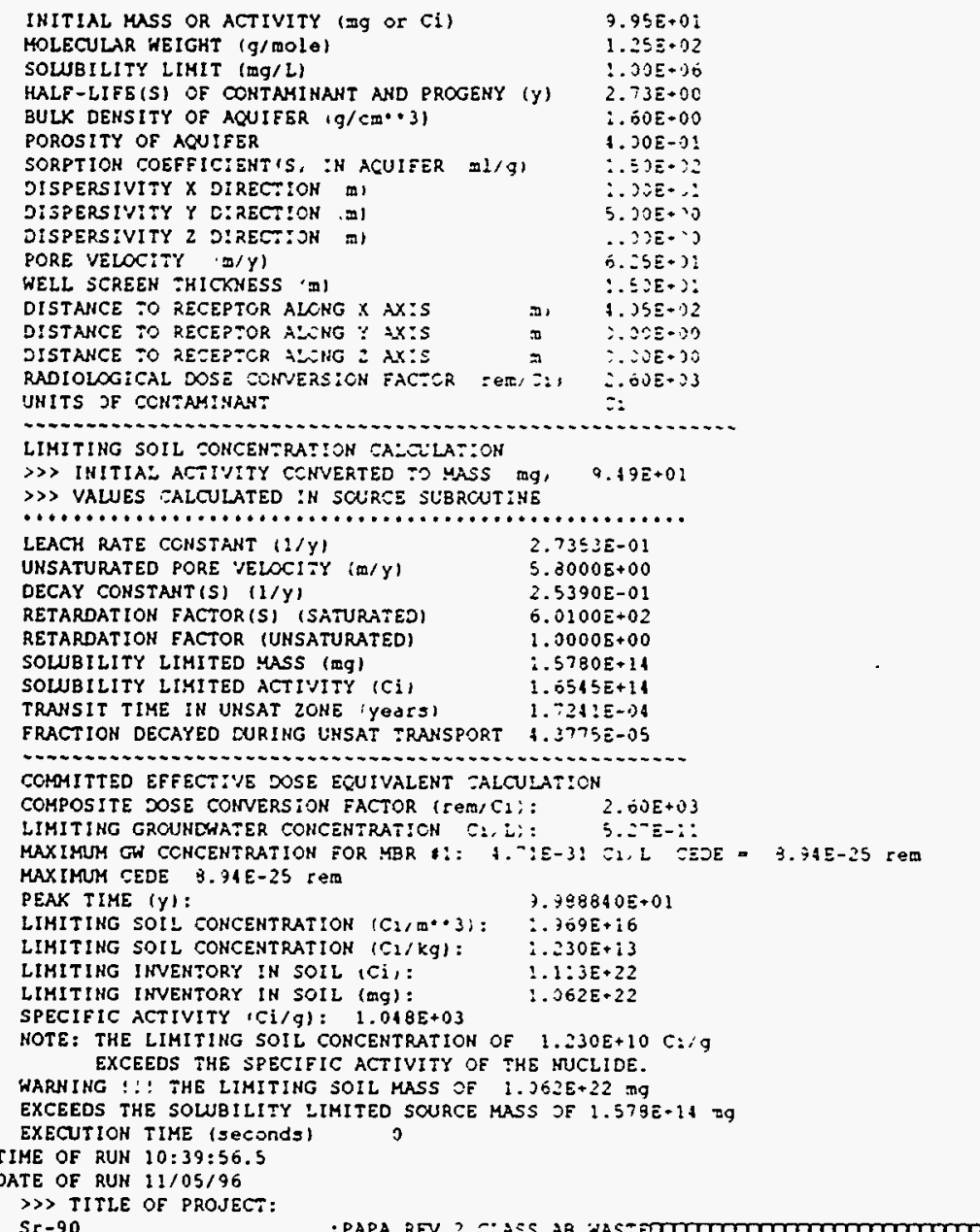

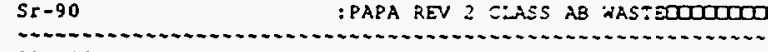

GAUSSIAN JUADRATURE SCLUTION

MODEL OPTIONS

IMODE: 1

KFLAG: 1 (0)CONC VS TIME: (1)PEAK CONC AND :TMITING SOIL FONC

IMODEL: 1 (1) SURF OR BURIED SOURCE: 21 POND SOURCE; $(3$, TABULATED SOURCE FUNCT ION ITYPE:0 (0) VERT AVG; (1) HON-VERT AVG: (2) AVG FROH O TO 20 $\gg$ IMPUT DATA

MURE of

LENGTH OF SOURCE PARALLEL TO GR FLOW (m)

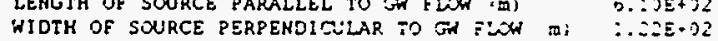

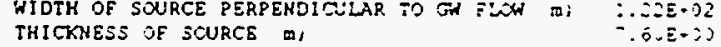

PERCOLATION RATE darCY ve: m/Yl

VCUMETR:C WATER CONTENT IN SCLRCE

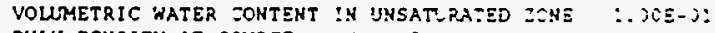

BULK DENSITY AT SCURCE $g / \mathrm{Cm}^{-\cdots 31} \quad: .2 \mathrm{SE} \cdot 32$

SORPTIOH COEFFICIEHT AT SCURCE ml.31

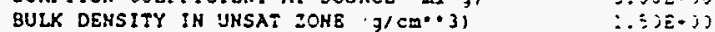

UNSATURATED 2 CHE THiCKNESS m, $m$,

SORPTIOH COEFFICIEHT IN JHSAT ZCNE mI 3 , $; .325+3$

OPTIONAL LOSS RATE CONSTANT FOR SCURCE,$\cdots-: \quad 3.202-20$

INITIAL YASS OR ACTIVITY IDg or $=2:$ :

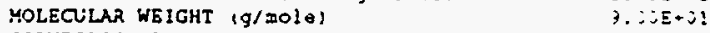

SOLUBILITY LIMIT (Mg/L)

HALE-LIFE(S) OF CONTAMINAMT AND PROGENY ! $: 9: E+3:$

BULK DENSITY OF ACUIFER, $9 / \mathrm{cm}^{*} \cdot 31 \quad \therefore .00 E+3 \hat{U}$

POROSITY OF ACUIEER $1.30 \mathrm{E}-31$

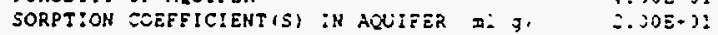

DISPERSIVITY $X$ OIREC::ON Wi

SISPERSIVITY 1 OIRECTION (m) $\quad 5.30 E+2 j$

DISPERSIVITY Z JIREC:!ON DI

D. $25 E-2:$

WELL SCREEN THICKNESS 'm? $\quad \therefore 50 E+01$

DISTANCE TO RECEPTOR ALONG $X$ AXIS $\mathrm{mI} \quad$ t.OSE+ 22

I) $3.30 \mathrm{E}+30$

DISTANCE TO RECEPTOR ALONG 2 AXIS mi $0.30 E+90$

RADIOLOGICAL DOSE CONVERSION FACTOR (rem/ $\mathrm{C}$ ) $\quad: .30 E+05$

UHITS OF CONTAMINANT

LIMITING SOIL COMCENTRATION CALCULATION 


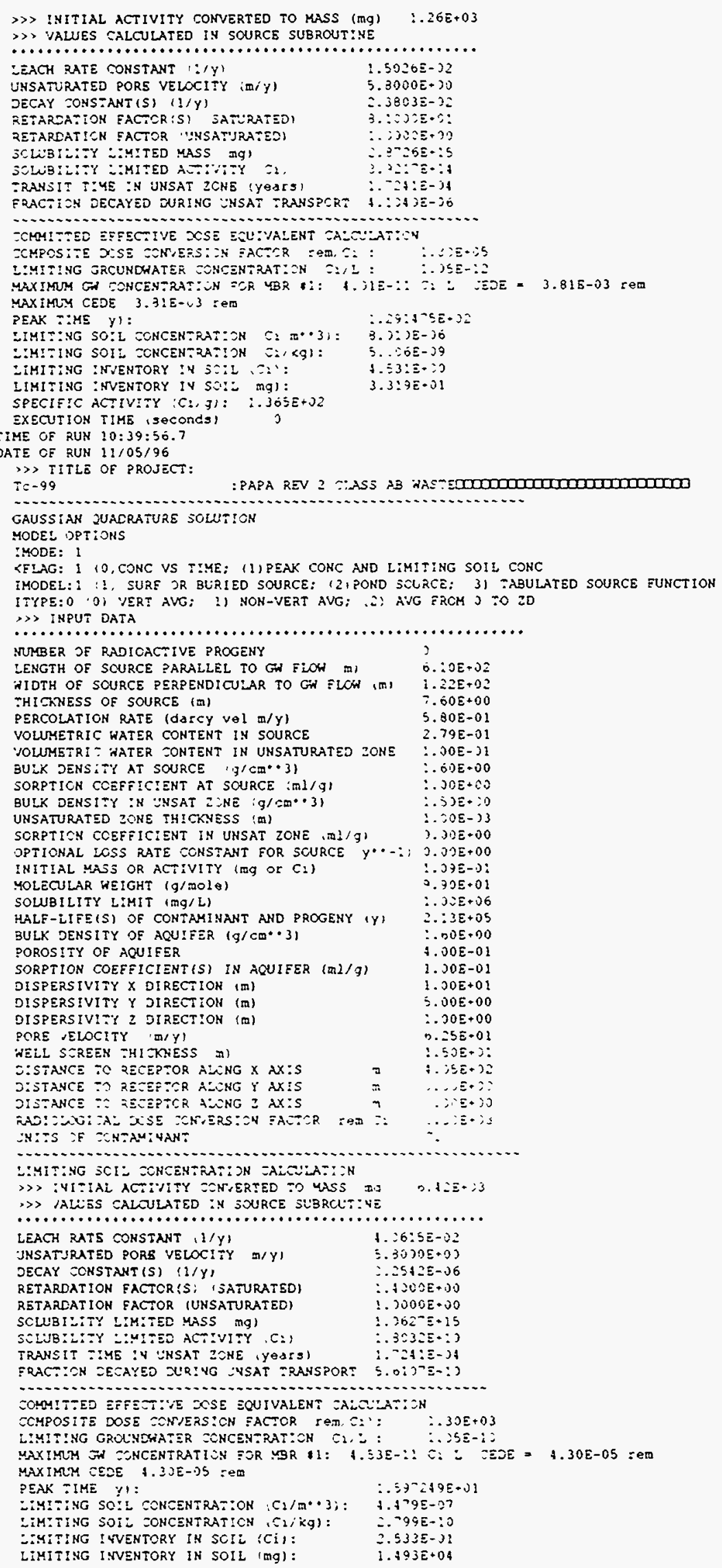




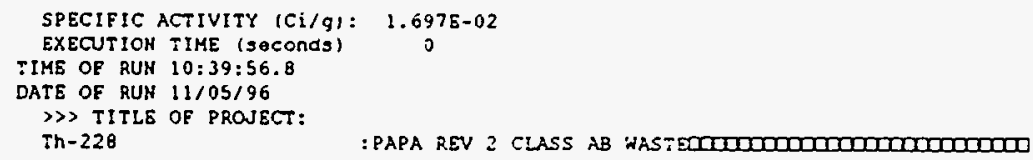




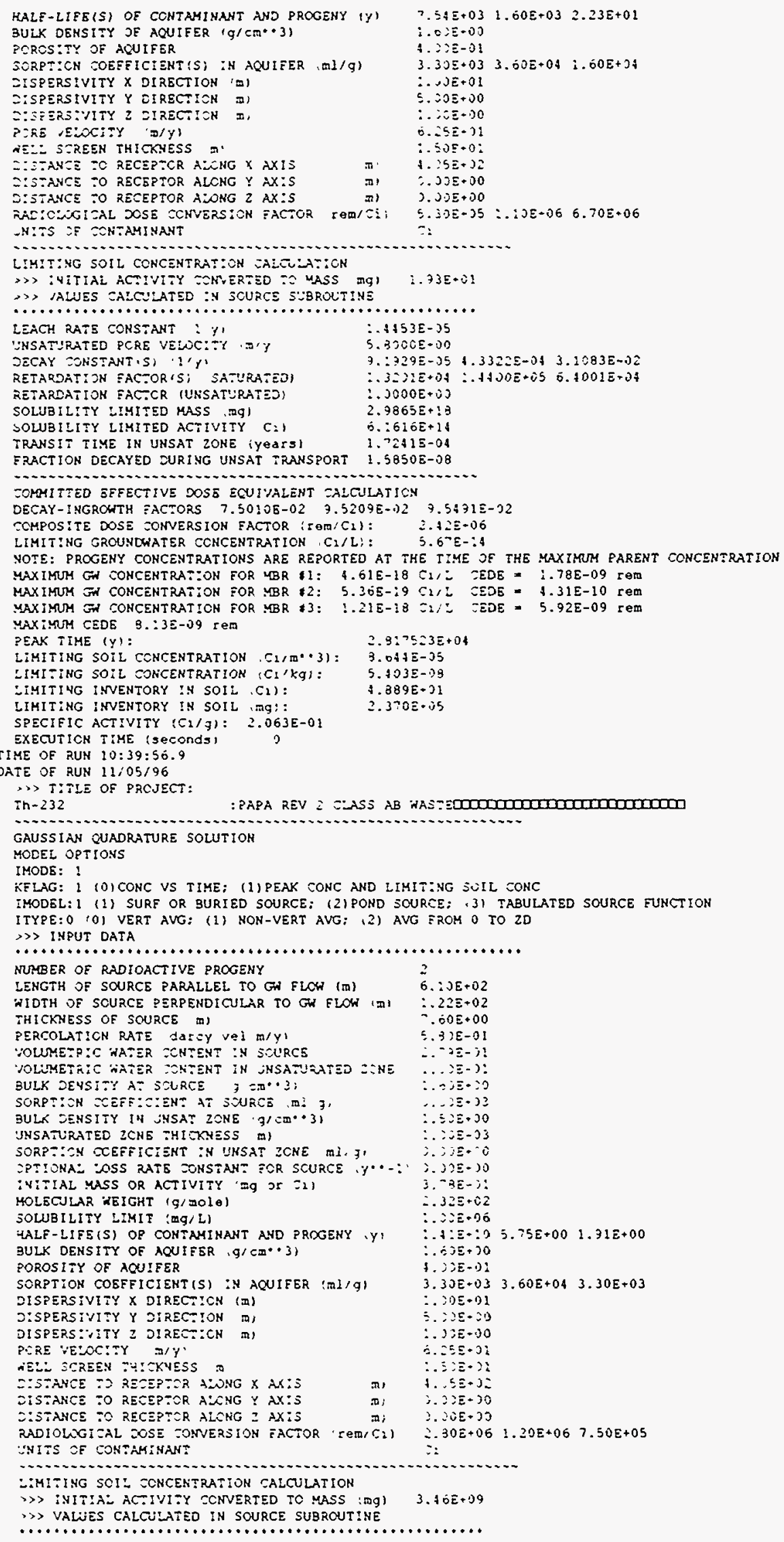




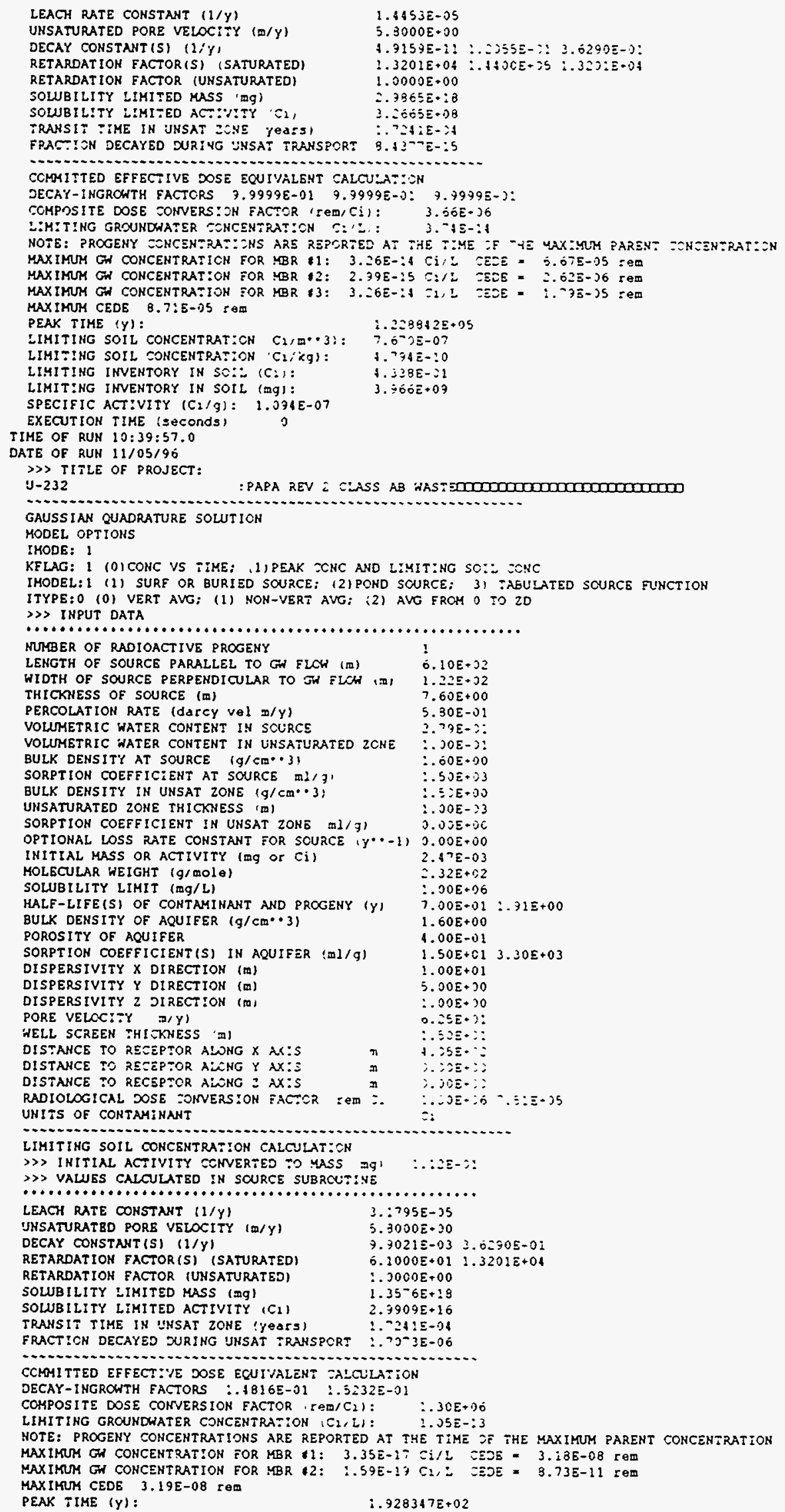




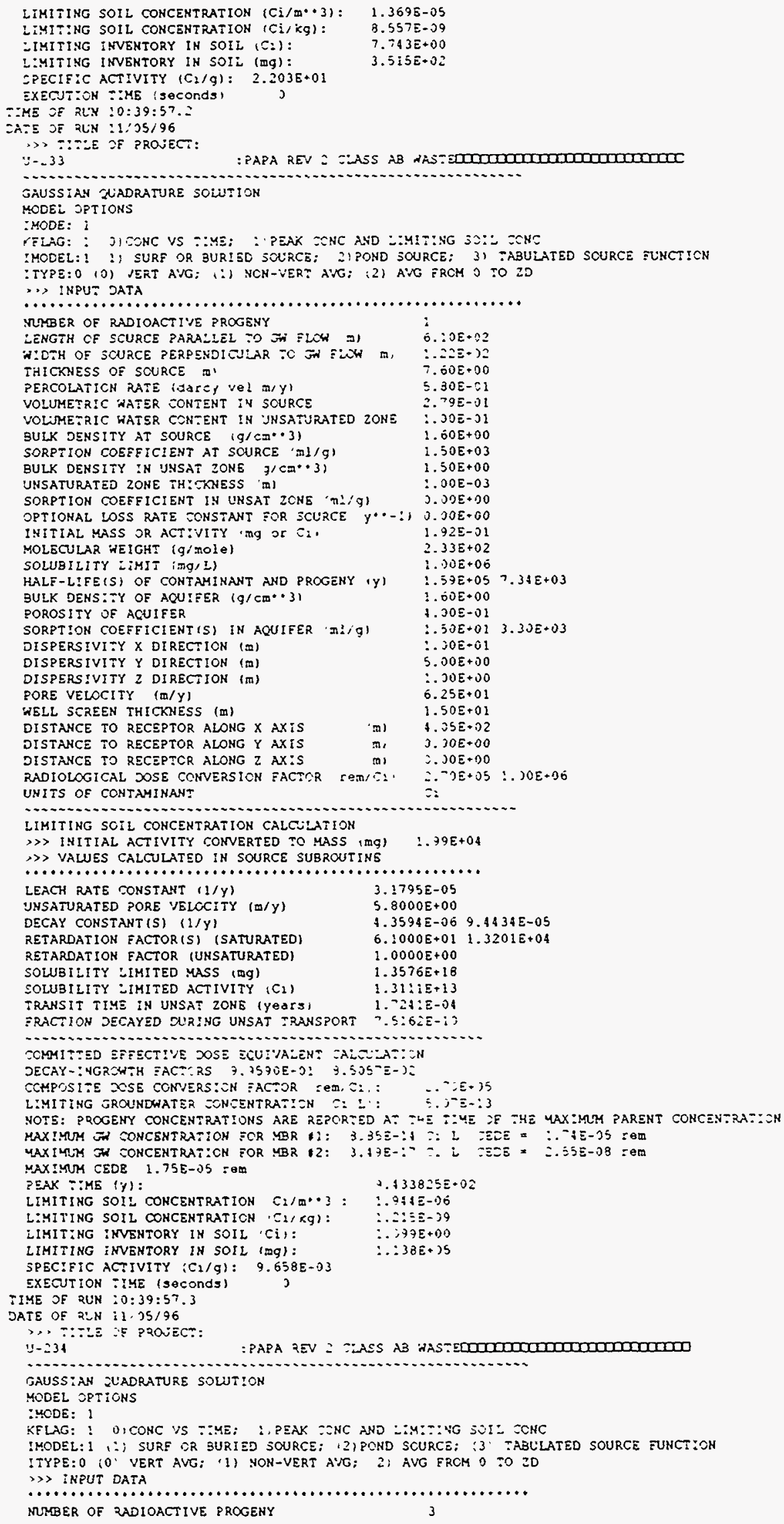




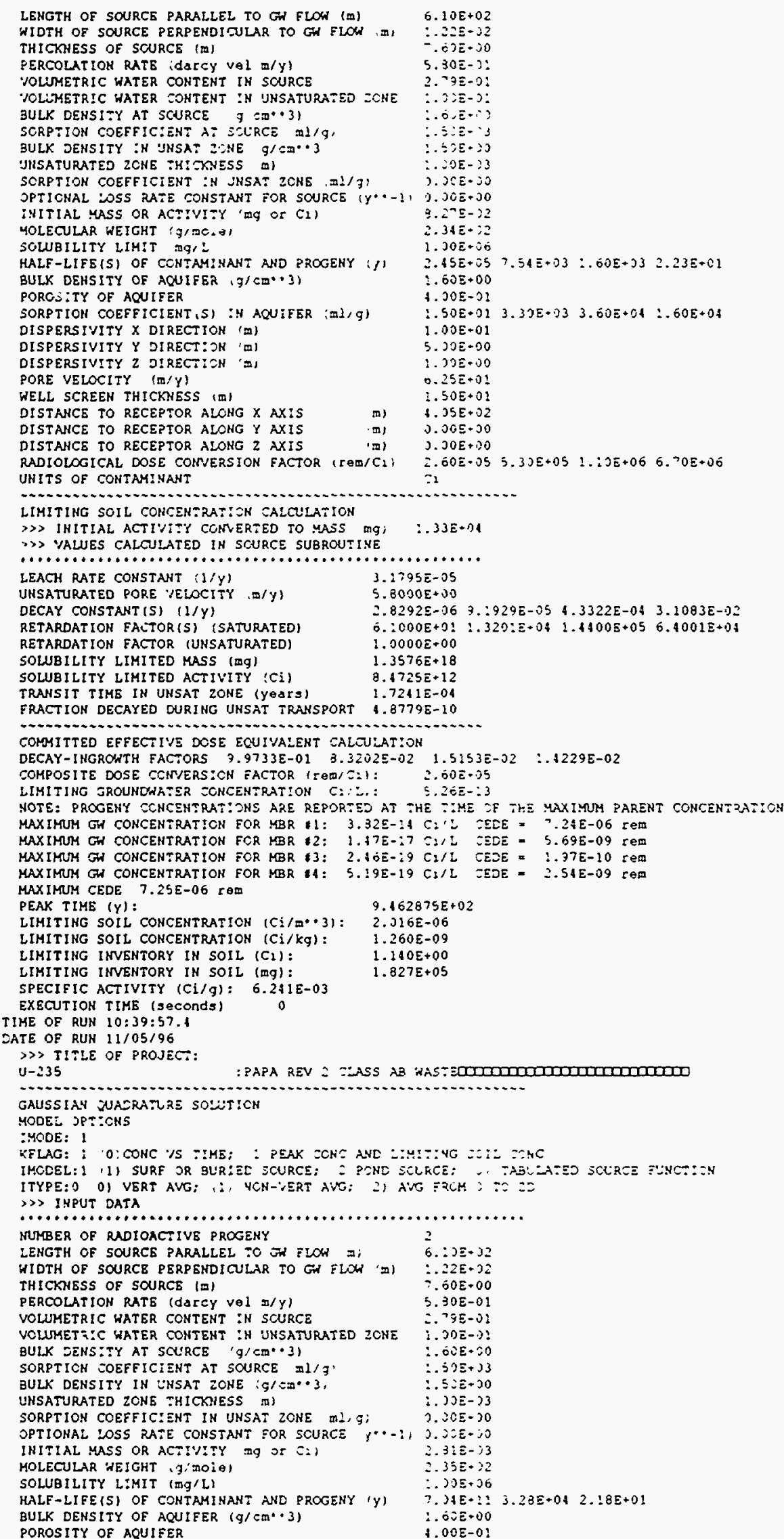




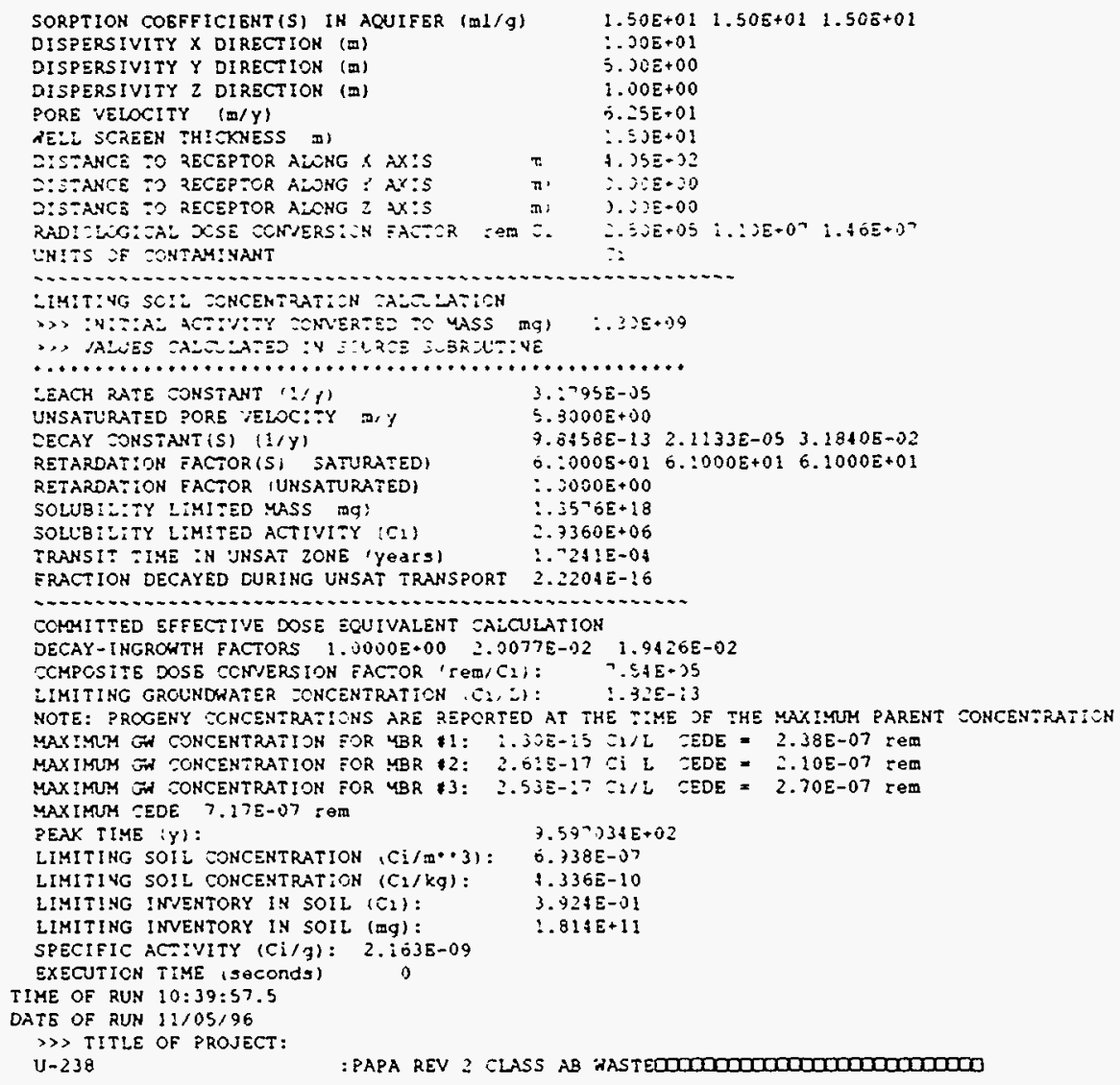




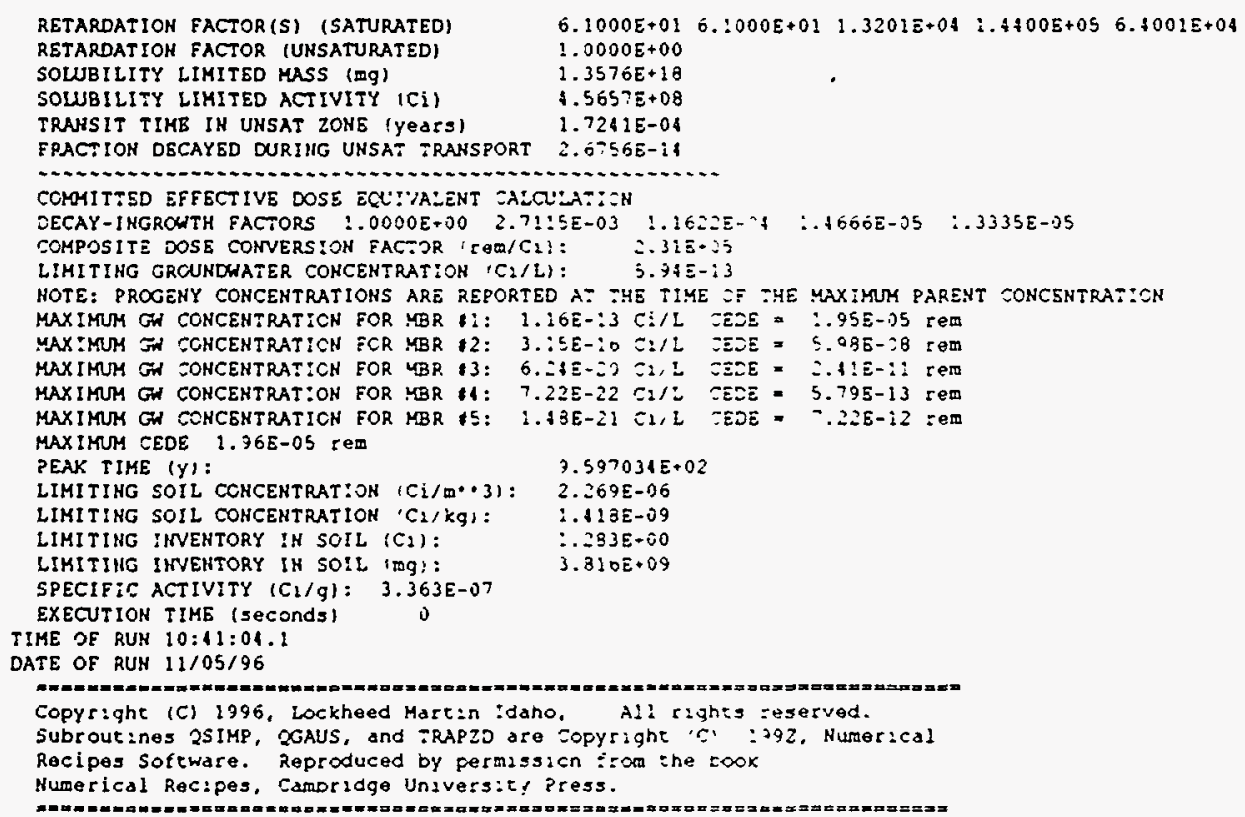




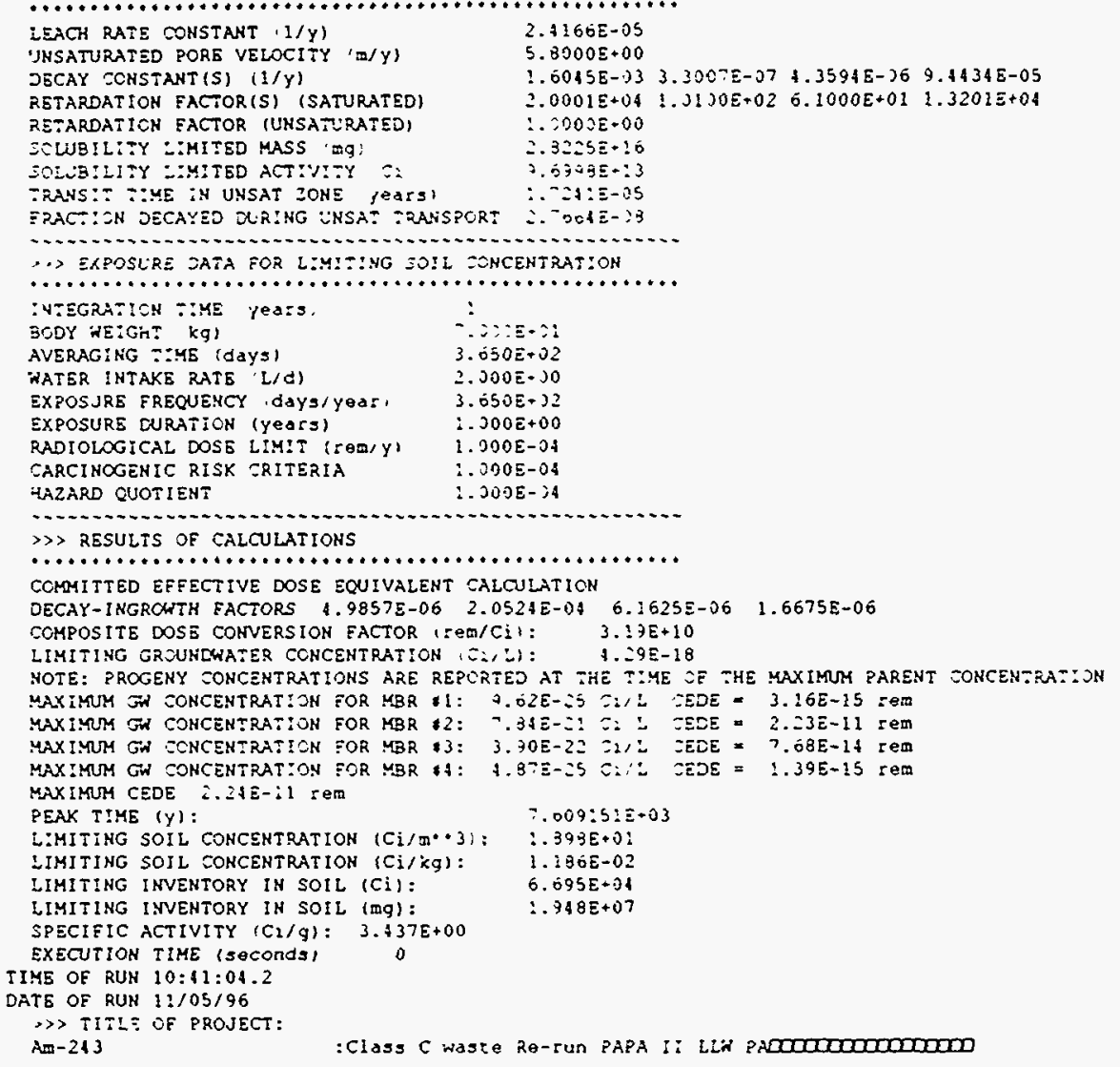




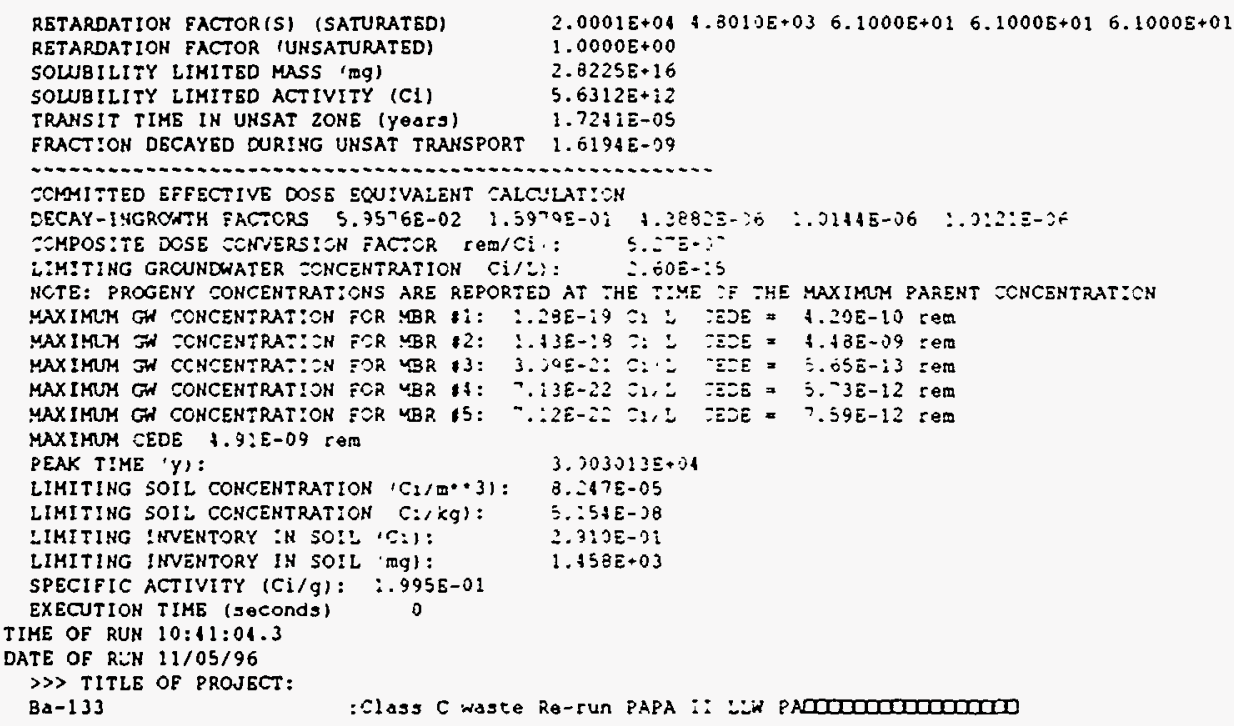

C........

DEITING SOIL CONCENTRATION CALCULATION

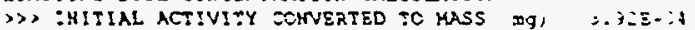

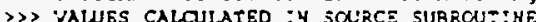




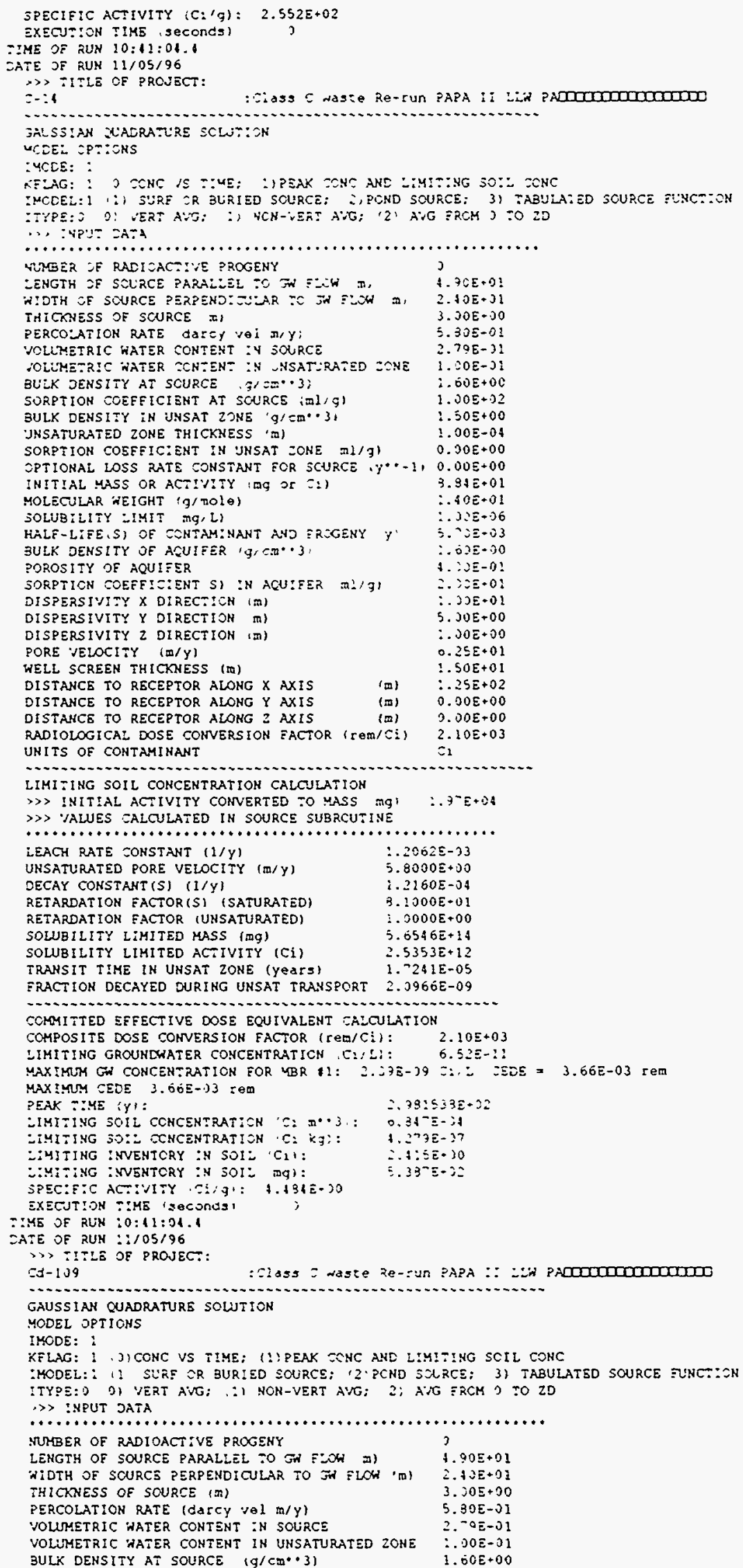




\begin{tabular}{|c|c|}
\hline SORPTION COEFFICIENT AT SOURCE $(\mathrm{ml} / \mathrm{g})$ & $2.00 \mathrm{E}+02$ \\
\hline BULX DENSITY IH UNSAT ZONE $(\mathrm{g} / \mathrm{cm} \cdots 3)$ & $1.508+00$ \\
\hline UHSATURATSD ZONE THICXNESS (D) & $1.205-24$ \\
\hline SORPTIOH COEFFICIENT IN UNSAT ZONE (ml/g) & $0.20 E=00$ \\
\hline OPTIONAL LOSS RATE CONSTANT FOR SCURCE $\left(\mathrm{Y}^{*}+1\right)$ & $0.00 E+00$ \\
\hline INITIAL MASS OR ACTIVITY img OS C: & $-.63 E-34$ \\
\hline YOLECULAR YEIGHT $(\mathrm{g} / \mathrm{mOl} \theta \mathrm{I}$ & $\therefore .27 \Xi-92$ \\
\hline SOUUBILITY LIMIT img/L) & $\therefore 305+30$ \\
\hline HALF-LIFE(S) OF CENTAMYNANT WND PRCGENY (Y' & $\therefore:=E-i J$ \\
\hline BULK JENSITY JE ACUIFER $\quad g^{\prime} \in \mathrm{En} \cdots 3$ & $\therefore 0: \Sigma-: 0$ \\
\hline POROSITY OF AQUIEER & 4. $20 \varepsilon-31$ \\
\hline SORPTICN COEFFICIENT(S) IN ACU:FER & 1. $00 \mathrm{E}-0$ ! \\
\hline JISPERSIVITY X OIRECTIOH 'WI & $\therefore 30 \mathrm{E}+2 !$ \\
\hline OISPERSIVITY Y SIRECTION mI & $5.20 E+20$ \\
\hline OISPERSIV:TY I JIRECT:ZN & $\therefore 33 E+22$ \\
\hline PORE VELOCITY II & $0.25 \varepsilon+3$ ? \\
\hline NELL SCREEN THICKNESS (MI & $\therefore 520-2:$ \\
\hline DISTANCE TO RECEPTOR ALCNG $X$ iX:S & $\therefore: \leq \varepsilon+22$ \\
\hline DISTAHCE TO RECEPTOR ALONG $Y$ AX:S & $3.308 \cdot 30$ \\
\hline DISTANCE TO RECEPTOR ALOHG $Z$ AX:S & $2.20 E-20$ \\
\hline $\begin{array}{l}\text { RADIOLOG:CAL DOSE CONVERSICN FACTAR rOT/SI, } \\
\text { UHITS OF COHTAYIHANT }\end{array}$ & $\therefore 23 E-34$ \\
\hline
\end{tabular}

LIMITING SOLL COHCENTRATION JALCLLAT:ON

$\gg$ IN!TIAL ACTIVITY CONVERTED TO MASS (mg) $2.74 E-04$ $\gg$ VALUES CALCULATED IN SOURCE SUBROUTINE

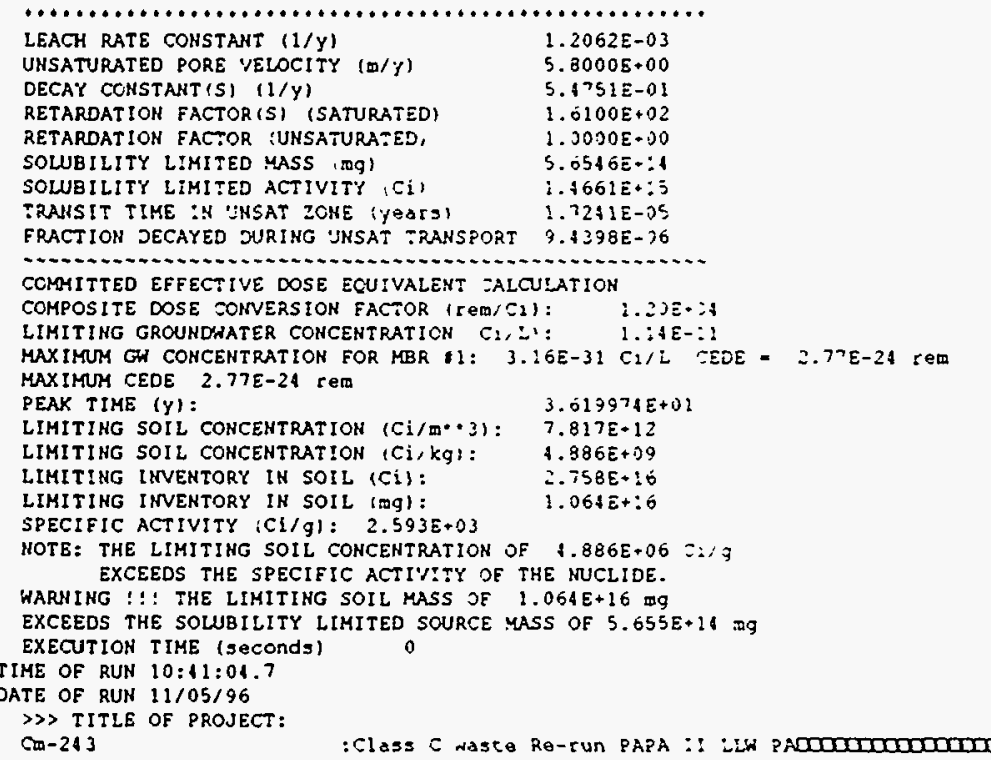

G

GAUSSIAN OUADRATURE SOLUTION

MODEL OPTIONS

IMODE: 1

KFLAG: I (0)CONC VS TIME: (1IPEAK CONC AWD LIMITING $30 ! \mathrm{L}$ CONC

IMODEL: 1 (I) SURF OR BURIED SOURCE: ¿iPCND SCLRCE: 2 TABULATED SOURCE EUNCTION

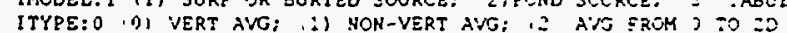

$\gg 1$ INPUT EATA

MUMBER OF RAD: JaCT:*E PROGENY

LENGTH OF SOLRCE PAFALLE: TO FW FLW nI

WIDTH OF SCURCE PERPENDICULAR TC 3 W ICH mi $2 .+3 E+3:$

THICKNESS OF SOURCS

PERCOLAT:ON RATS , dAICY VEl W/Y!

VOLMETRTC WATER COTTEN

IM SCLRCE $:-9 \equiv-j:$

VOUMETRIC WATER CONTENT IN JNSATJRATED ZENE $\quad 3$ JEE-;:

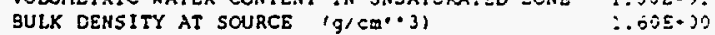

SORPTION COEFFICIENT AT SOURCE Wl'gl

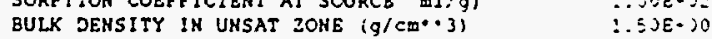

INSATURATSD ZONE THICKNESS (II)

SORPTION COEFEICISNT IN SNSAT ZONE IMI/g), $30 E+30$

OPTIONAL LOSS RATE CONSTANT FOR SOURCE (Y $\cdots-1), 30$ ) 30

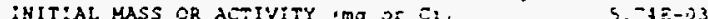

MOLECULAR WETGH' $9 /$ TOlOI

MOLECULAR WE:GHZ ig/TOLOU

SOLUBILITY L:MIT MG/D)

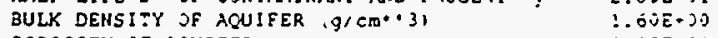

POROSITY OF AQUIFER

SORPTION COEFFICIENTISI :N ACUIFER a.J.

DISPERSIVITY $X$ OIRECTION $(\mathrm{m}) \quad$ O.JOE+

DISPERSIVITY $Y$ DIRECTION DI $5.00 E+00$

DISPERSIVITY Z DIRECTION (m)

WELL SCREEN THICNNES (m) $1.25 E+91$

WELL SCREEN THICNNESS (m) $\begin{array}{lll} & 1.50 E+01 \\ \text { DISTANCE TO RECEPTOR ALONG X AXIS (m) } 1.25 E+02\end{array}$

DISTANCE TO RECEPTOR ALONG $Y$ AXIS $\quad(\mathrm{m}) \quad 0.00 E+00$ 


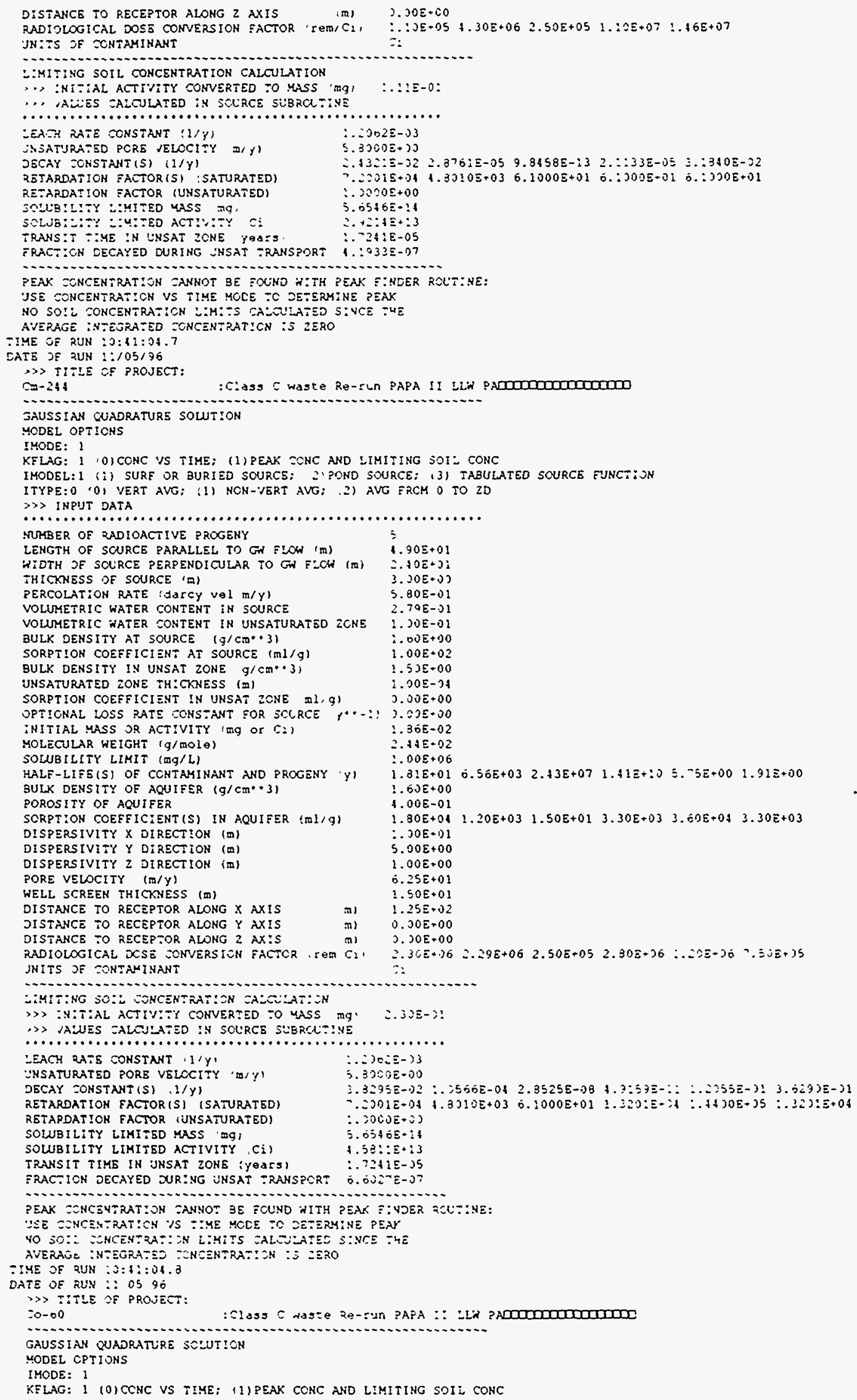


IHODEL: 1 (1) SURF OR BURIED SOURCE; (2) POND SOURCE; 3; TABLLATED SOURCE FUNCTION ITYPE: 0 (0) VERT AVG; (1) NON-VERT AVG; 121 AVG FRCM 0 TO $2 D$ \\ INPUT DATA

\begin{tabular}{|c|c|}
\hline UMBER OF RADIOACTIVE PROGENY & J \\
\hline HOTH OF SOURCE PARALLEL TO GN ELOW (m) & $1.305+91$ \\
\hline IDTH OF SOURCE PERPENDICULAR IO FN ELW & $=10 \equiv \cdot 3:$ \\
\hline IICKNESS OF SCURCE : & (.X:三*;) \\
\hline ERCOLAT:ON RATE (daTCY VeI $\pi / y)$ & $\vdots .3 \vdots \equiv-?$ \\
\hline WLLMETRIC WATER SCNTENT IN SOURCE & $\ldots-\lambda \equiv-:$ \\
\hline VOLUKETRIC HATER CCNTENT IN UNSATERATED ICNE & $\therefore ; \vdots \equiv-i:$ \\
\hline BULK DENSITY AT SOURCE $(\mathrm{g} / \mathrm{cm} \cdots 3 ;$ & $\therefore 0 \hat{0} \equiv+20$ \\
\hline SORPTION COEFFICIENT AT SCURCE $=1, \exists$ I & $\therefore 3,2=32$ \\
\hline BULK DENSITY IN JNSAT ZONE + $91=3^{\prime} \cdot 3^{\prime}$ & $1.52 E \cdot \div 0$ \\
\hline UHSATURATED ZCNE TH:ZKNESS ' & i. ว?E-24 \\
\hline SORPTIOH COEFFICIIHT :N UNSAT ZCNE ML/GI & $2.23 E+30$ \\
\hline OPTIOHAL LOSS RATE CONSTANT FOR SCURCE $y^{*}-1$, & $3.008+30$ \\
\hline IHIT:AL MASS OR ACTIVITY Ing OI $: 2$. & $-\because 5 \Sigma \cdot 35$ \\
\hline MOLECULAR NEIGHT $g /$ mOIOI & $0.005-1:$ \\
\hline SOUBILITY LIMIT (mg/L) & $\therefore: Y E \cdot 96$ \\
\hline HALF-LIFE(S) OF EONTAMYNANT AHE PROGENY & $5.75 E+20$ \\
\hline BULK DENS:TY OF AQUIFER $(j,=\mathrm{m} \cdots 3)$ & $\therefore 60 \mathrm{E} \cdot 2 \mathrm{~J}$ \\
\hline POROSITY OF AQUIFER & 4.JPE-): \\
\hline SOKPTION COEFFICIENT(S, :K ACLIFER (TI/g) & $1.30 E+03$ \\
\hline DISPERSIVITY $X$ DIRECTION $\{=1$ & $\therefore .305+01$ \\
\hline DISPERSIVITY Y DIRECTION (⿴) & $5.20 E+00$ \\
\hline OISPERSIVITY 2 DIRECTION (D) & $\therefore .00 E+00$ \\
\hline PORE VELOCITY $(m / Y)$ & $6.25 E+0:$ \\
\hline WELL SCREEN THICONESS .II & $: .5 \supset E+01$ \\
\hline DISTANCE TO RECEPTOR AWHG $X$ AX:S & $\therefore .25 E \cdot 32$ \\
\hline DISTAMCE TO RECEPTOR ALONG Y AX:S & $0.20 E+30$ \\
\hline DISTANCE TO RECEPTOR ALUNG I AK:S & $3.30 \bar{\varepsilon}+33$ \\
\hline RADIOWGICAL DOSE CONVERSION FACTOR & $2.00 \mathrm{E}+04$ \\
\hline UNITS OF CONTAMINANT & $c:$ \\
\hline
\end{tabular}

LIMITING SOIL CONCENTRATION CALCJLAT:ON

$\gg$ INITIAL ACTIVITY CONVERTED TO MASS (mg) $7.17 E+35$ $\gg$ VALUES CA:CULATED IN SCLRCE SUBREUTINE

$\gg$ VALUES CALCULATED IN SCLRCE SUBRLUTIN

\begin{tabular}{|c|c|}
\hline $\begin{array}{l}\text { LEACH RATE CONSTANT }(1 / Y) \\
\text { UNSATURATED PORE VELOCITY (m/Y) }\end{array}$ & $\begin{array}{l}1.2062 E-03 \\
5.8000 E+00\end{array}$ \\
\hline DECAY CONSTANT (S) $(! / Y)$ & $i .2055 E-01$ \\
\hline RETARDATIOH EACTOR(S) (SATURATSD) & $5.20108+03$ \\
\hline RETARDATION FACTOR (UNSATURATED) & $1.0000 E=00$ \\
\hline SOWBILITY LIMITED MASS (mg) & $5.6546 E+14$ \\
\hline SOWBILITY LIMITED ACTIVITY (CI) & $5.8643 E+11$ \\
\hline TRANSIT TIME IN UNSAT ZONS yearSI & $1.7241 E-35$ \\
\hline FRACTION DECAYED DURING UNSAT TRANS & $2.0784 E-36$ \\
\hline
\end{tabular}

SRACTION DECAYED DURING UNSAT TRANSPCRT $2.0781 E-36$

PEAK CONCENTRATION CANNOT BE FCUND HITH PEAK FINDER RCLT:NE:

USE CONCENTRATION VS TIME MODE TO DETERMIME PEAK

HO SOIL CONCENTRATION LIMITS CALCUIATED SINCE THE

AVERAGE INTEGRATED CONCENTRATION IS ZERO

TIME OF RUM 10:41:04.8

DATE OF RUN $11 / 05 / 96$

\ TITLE OF PROJECT:

Cs -134

:Class C waste Re-run PAPA 1i ïn PAD0000000000000

GAUSSIAN QUADRATURE SOLUTION

MODEL OPTIONS

IMODE: 1

KFLAG: 1 (0) CONC YS TIME: 11)

IMODEL: 1 (1) SURF OR BURIED SCURCE; 2) POND SOURCE; 3, TABULATED SOURCE FUNCTION

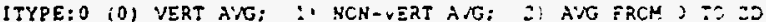

$\gg$ INPUT DATA

MUMBER JF RADIOAC:.... PROGENY

LENGTH OF SCLREE PARALESL :O GN FLOA $x$,

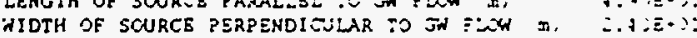

THICKMESS OF SCURCE (m)

PERCCLATION RATE, dATCY VOl III

VOUMETRIC HATER CONTENT IN SQRRE

VOLUMETRIC WATER CONTENT IN JNSATURATEO IJNE $: . V E-;$

BULK DENSITY AT SOURCE $, g / \mathrm{cm} \cdots 31 \quad \vdots 0: E-2 J$

SCRPTIOH COBFFICIEHT AT SOURCE (mi/g)

SORPTIOH COBFE ICIBHT AT SOURCE 'GOI/g)

$\therefore .5, \Sigma+30$

UNSATURATED ZOHB IHICKNESS (OO)

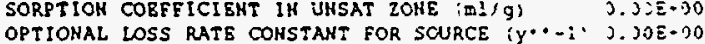

INITIAL YASS OR ACTIVITY :Ing OI $C_{2}$ )

MOLECU:AR WEIGHT (g/mOL)

SOLUB:L:TY LIMIT, $\mathrm{gg} / \mathrm{L}$

HALE-LIFE,S) OF EONTAMIHANT AND RROGENY Y1

BULK DENSITY OF ACUIFER $/ g / \mathrm{cm}^{*} \cdot 3, \quad: .00=20$

POROSITY OF ACUIFER T.JOE-01

POROSITY OF ACUIFER

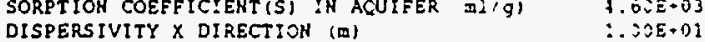

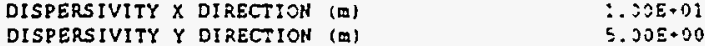

DISPERSIVITY $Z$ DIRECTION (m)

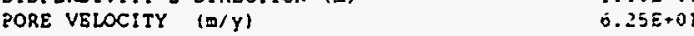

HELL SCREEN THICKNESS (W) 1.5$) E+01$

DISTANCE TO RECEPTOR ALOHG X AXIS (II) $1.25 E+02$

DISTANCE TO RECEPTOR ALONG Y AXIS (m) $0.00 E+00$ 


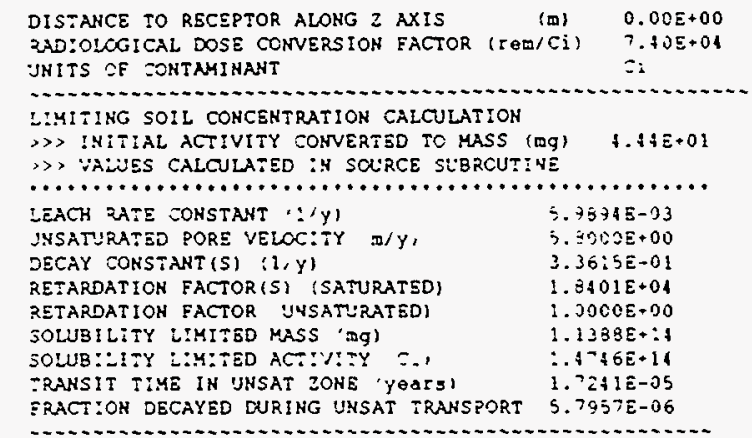

PEAK CCNCENTRATION GANHOT BE ECUHD WITH PEAK I:NCER ROUTINE:

JSE CONCENTRATION IS TIME MCDE TO VETERMINE PEAK

NO SO:L CONCENTRATION LiMITS CALCJLATEO SINCE THE

AVERAGE INTEGRATSO CONCENTRATION IS ZERO

T:ME OF RUN 10:41:04.9

SATE OF RUN ::105/96

\ IITLE OE PROJECT:

$23-137$

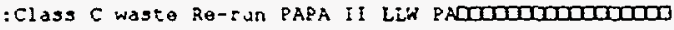

GAUSS:AN GUAORATURE SOUTION

MODEL OPTIONS

IMODE: :

KELAG: I (0)CONC VS TIME; (1) PEAK CONC AND LIMITING SOIL CONC

IMCDEL: 1 (i) SURF OR BURIED SOURCE: 2; POND SCURCE: 3) TABULATED SOURCE EUNCTION

ITYPE:0 (0) VERT AVG: (1) NCN-VERT AVG; (2) AVG FRCM $O$ TO $Z D$

$\gg$ INPUT DATA

MURBER OF RADIOACTIVE PROGENY

IENGTH OF SOURCE PARALLEL TO GN FLOW (II) I.

WIDTH OF SOURCE PERPENDICULAR TO TU ELOH (A) $2.40 E+01$

WIOTH OF SOURCE PERPENDICUI

PERCOLATION RATE (dATCY VEl TR/Y) $5.30 E-01$

VOLLMETRIC NATSR CONTEHT IN SCURCE $2.79 E-01$

VOLUMETRIC WATER CONTENT IN UNSATURATED 2OHE 1.DOE-01

BULX DENSITY AT SOURCE $(\mathrm{g} / \mathrm{CR} \cdots 3) \quad 1.60 E+00$

SORPTION COEFFICIENT AT SOURCE $(\mathrm{ml} / \mathrm{g}) \quad 2.00 E+01$

BULK DENSITY IN UNSAT ZOHE ( $q /=2 \cdots 3$ ) $\quad: .50 E+00$

UNSATURATED ZONE THICKNESS (m) $2.00 E-94$

SORPTION COEFFICIENT IN UNSAT ZONE $\mathrm{ml/g}$ ' $0.00 E+00$

OPTIOHAL LOSS RATE CONSTANT GOR SOURCE $Y \cdots-1 ; 0.00 E+00$

INITIAL MASS OR ACTIVITY img Or $C_{11}$, $1.23 \equiv+02$

$\begin{array}{ll}\text { INITIAL MASS OR ACTIVITY img OF C1) } & 1.23 \Xi+02 \\ \text { MOLECULAR HEIGHT (g/mole) } & 1.375+02\end{array}$

$\begin{array}{ll}\text { MOLECULAR HEIGHT }(\mathrm{g} / \mathrm{mole}) & 1.37 \mathrm{l}+02 \\ \text { SOLUBILITY LIMIT }(\mathrm{ag} / \mathrm{L}) & \vdots .008+06\end{array}$

SOLUBILITY LIMIT (Rg/L)
HALE-LIFE(S) OF CONTAMINANT AND PROGENY (Y) $3.00 E+01$

BULK DENSITY OF AQUIFER $(\mathrm{g} / \mathrm{cm} \cdots 3) \quad 1.60 \mathrm{E}+00$

POROSITY OE AQUIFER

SORPTION COEFFICIENT(S) IN AQUIFER (MI/g) $4.60 E+03$

DISPERSIVITY X DIRECTION (m) $1.00 E+01$

DISPERSIVITY $Y$ DIRECTION (D) $5.00 E+00$

DISPERSIVITY 2 OIRECTION (m) $1.00 E+00$

PORE VELOCITY ( $\mathrm{m} / \mathrm{Y}) \quad 6.25 E+01$

WELL SCREEN THICKNESS (⿴) $1.50 E+01$

DISTANCE TO RECEPTOR ALONG $X$ AXIS (i) $1.25 E+02$

DISTANCE TO RECEPTOR ALONG $Y$ AXIS in $0.00 E+00$

JISTANCE TO RECEPTOR ALONG $Z$ AXIS m) $0.0 \mathrm{JE}+00$

PADIOLOGICAL DOSE CONVERSION FACTOR :

ZN:TS OF EANTAMINANT

$\therefore$

IIMIT:NG SOI: ECNCENTRAT:JN GALOLIAT:YH

\> INITIAL ACIVITY CONVERTED TO MASS Tg! $4.35 E+23$

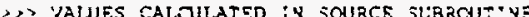

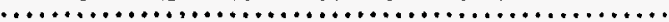

LEACH RATE CONSTAHT .1/YI $5.9894 E-33$

JNSATURATED PORE VELOCITY $: m / Y, \quad 5,30305 \cdot 20$

DECAY CONSTANT(S) (1/Y) $\quad \because 3: 0 S E-02$

RETARDATION FACTOR(S) ISATURATSO)

PETAPJATION FACTOR IUNSATURATEDI $\quad \therefore 3003 E+30$

SOLUBILITY LIMITED MASS (Mg) $: 2388 E+14$

SOUBIL:TY LIMITED ACTIVITY (Ci) $7.9137 E+i 2$

TRANSIT TIME IN UNSAT ZONE (Y9arS) 1.7241E-J5

SRACTION OECAYED DURING UNSAT TRANSPCRT $3.0836 \mathrm{E}-\mathrm{Q}$ ?

PEAK COHCENTRATICN CANNOT 3 E FOUND AITH PSAK F.NDER RCLTINE

JSE CONCEYTRATICH VS TIME MODE TO IETEPM:NE PSAK

MC SO:L GENCENTRATEON Z:MITS CAUCULATED S:NCE THE

AVERAGE VNIEGRATES IENCENTRAT:ZN :S ZERO

T:ME OF RUN :0:4::04.3

DATE OF RUY $: 2,35,96$

P TIT:E OF PROJECT:

Fe-55

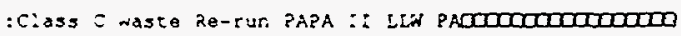

GAUSSIAN OUADRATURE SOLUTION

MODEL OPTIONS

IMODE: 1

KFLAG: 1 (0) CONC VS TIME: (1)PEAK CONC AND LIMITING SOIL CONC 
IMODEL: 1 (1) SURF OR BURIED SOURCE; (2) POND SOURCE: (3) TABUEATED SOLRCE FUNCTION ITYPE:0 (0) VERT AVG: (1) NON-VERT AVG: 121 AVG FRCM $0: 0=0$ \IINPLT DATA

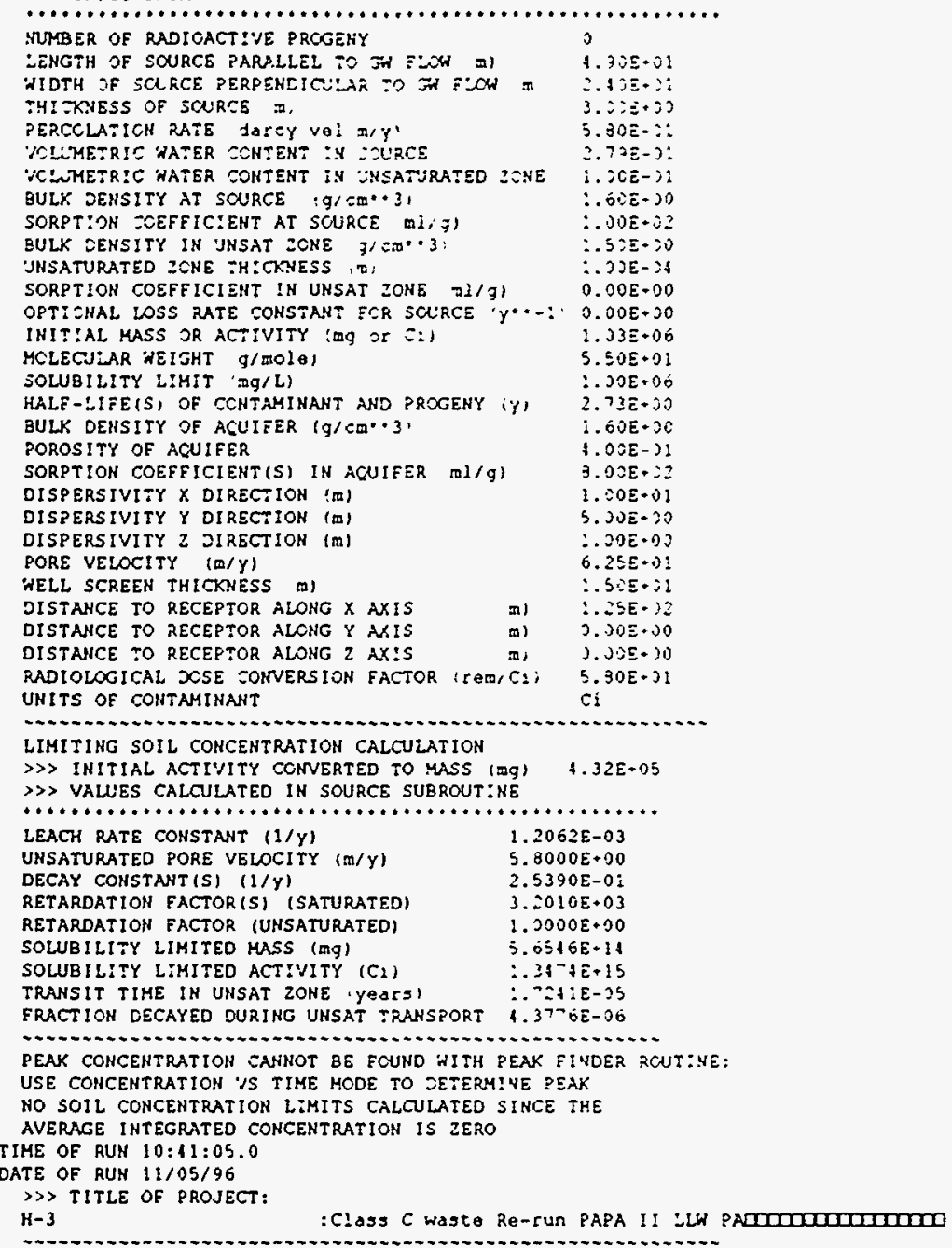

GAUSSIAN QUADRATURE SOWTION

MODEL OPTIOHS

IMODE : 1

KFLAG: 1 (0)CONC VS TIME; (1) PEAK CONC AND :IMITING SOIL CONC

IMODEL: 1 (1) SURF JR BURIEO SOURCE; (2) ROND SOURCE; (3) TABULATED SOURCE FUNCT:ON ITYPE:0 (D) VERT AVG: (1) WON-VERT AVG; il AVG FRCM $): 0$ :0

$\gg$ INPUT $2 A: A$

NUMBER SE RADIOACT:VE PROGENY

LEMGTH OF SOURCE PARALLEL TO TN FLZW O

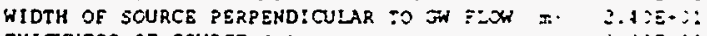

THICKNESS OF SOURCE (m)

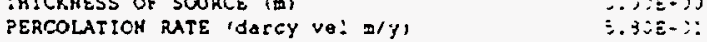

PERCOLATIOH RATE 'dATCY ve: m/Yl
YOLLMETRIC WATER TONTEN: :N SOLRCE

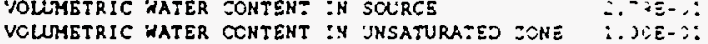

BULK DENSITY AT SOURCE $(g / 20 \cdots 3)$ O.

SORPTION COEFF:CIENT AT SOURCE MI'gl ;.3LE.?

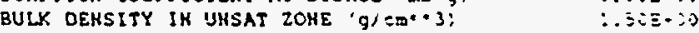

UNSATURATED ZONB THICKNESS (m)

SORPTION COEFPICIENT IH UNSAT ZONE (mi/g) $3.03 E+100$

OPTIONAL LOSS RATE CONSTANT FOR SOURCE $(\mathrm{y} \cdots-1) \quad 0.02 E-50$

INITIAL MASS OR ACTIVITY ing or CE,

MOLECULAR NEIGHT $(\mathrm{g} /$ MOlel

SOUB:L:TY LIMIT MG/LI

HALE-LIEE,SI OE CONTAMINANT AND PROGENY $Y ; \quad: 23 \bar{z}+2:$

BULK DENSITY OF AQUIFER $(\mathrm{g} / \mathrm{cm} \cdots 3) \quad \vdots 00 E+j)$

POROSITY OF AQUIFER

SORPTION COEFEIC:ENT(S) :N ACUIFER $\mathrm{ml} / \mathrm{g}$ ) $0.20 E \cdot 30$

OISPERSIVITY $X$ DIRECTION - Mा

DISPERSIVITY Y DIRECT:ON III

DISPERIVITY Y DIRECT:ON $3.3 \mathrm{~mL}=20$

$\therefore 33 E+30$

PORE VELOCITY (W/Y)

WELL SCREEN THICKNES (m)
OISTANCE TO RECEPTOR ALONG X AXIS m) $1.5 \vdots+01$
DISTANCE TO RECEPTOR ALOHG Y

$\begin{array}{llll}\text { DISTANCE TO RECEPTOR ALONG } X \text { AXIS } & \text { m) } 1.25 E+02 \\ \text { DISTANCE TO RECEPTOR ALONG } Y \text { AXIS } & \text { m) } 0.00 E .00\end{array}$ 


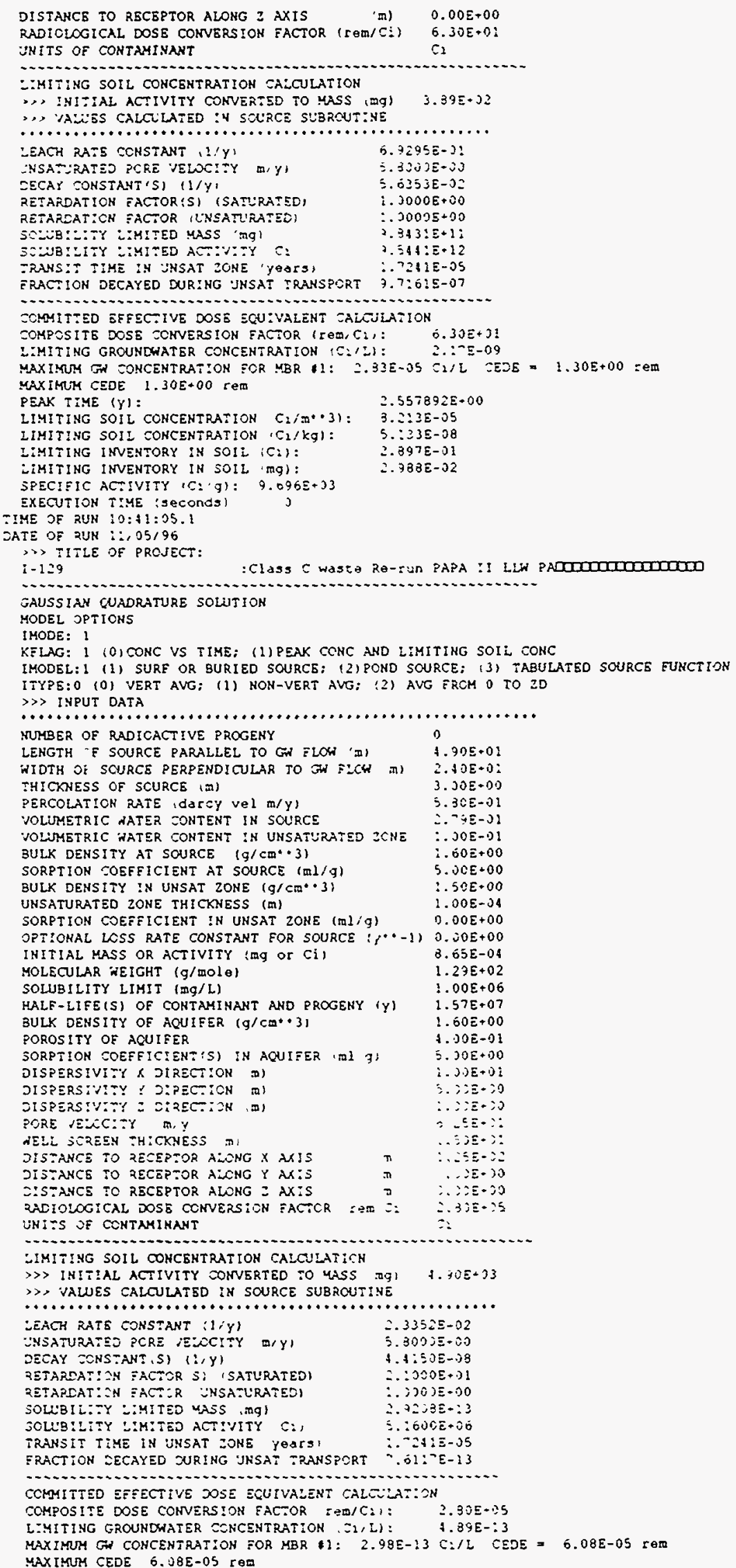


PEAK TIME $(y)$ :

\$.23:E-07

LIMITING IMVENTORY IN SOIL (Ci):

DIMITING ITVEHTORY IN SOIL (mg):

SPECIFIC ACTIVITY (C), g1: :-767E-04

SPECIFIC ACTIVITY $\left(C_{2}, g 1:\right.$ : ${ }^{-7} 67 E-04$
EXECUT:CH TIME seCONOS,

TIME JF RLH $13: 1:: 05.2$

DATE SF RUN $11,05 / 96$

$\gg$ TITLE OF PRCIEC.

Nb-9i

GAUSSIAH OUADRATURE SOUUT::H

MODEL JPT:ONS

IMODE: 1

KFLAG: 1 (0) CONC IS TIME; 11PEAK CCMC AMD :TMITING SOIL COHC

IMODEL: 1 (1) SURF OR BURIED SCURCE: (2) RCND SOURCE: (3) TAGULATSD SOURCE FUNCTION

ITYPE:O (D) VERT AVG: : , MON-VERT ANG; 21 AVG ERCM $O$ TO $2 D$

\INPUT DATA

WUMBER OF RADIOACTIVE PROGENY

LENGTH OF SCURCE PARALDEL TO GN ELOW 'ml

WIDTH OF SCURCE PERPENDICULAR TO GH FLON (m) 2.40E-0!

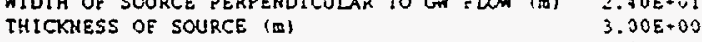

PERCOLATION RATE (dATCY $/ O 1 \mathrm{~m} / \mathrm{Y}$ )

VOLUMETRIC WATER CONTENT IN SOURCE

BULK DENSITY AT SOLRCE $\mathrm{g} / \mathrm{em} \cdots 3) \quad: .00 \mathrm{3}+00$

SORPT:ON COEFFICIENT AT SCURCE LI,,$\quad$ i. $30 E-03$

BULK DENSITY IN UNSAT ZCNE $(g / \in \mathbb{C} \cdots 3)$

UNSATURATED ZOHE TH:CXNESS 'TI

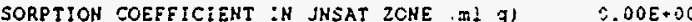

LOSS RATS CONSTANT FOR SCURCE $(Y \cdots-1) \quad 0.00 E * 00$

INITIAL MASS OR ACTIVITY (ROg OF Cil

MOLECULAR KEIGHT (g/mOle) $3.10 E+0$ ?

SOLUBILITY LIKIT ' $\mathrm{gg} / \mathrm{L}$ ) $\quad$. J0E.06

HALF-LIFE (S) OF CONTAHINANT AND PROGENY (Y) $2.00 E+04$

BULK DENSITY OF ACUIFER : $g / \mathrm{CM}^{*+3)} \quad 1.00 E+00$

POROSITY OE AQUIEER

SORPTION COEFFICIENT (S) IN ACUIFER (ml/q) $5.50 E+02$

DISPERSIVITY $X$ DIRECTION (m)

OISPERSIVITY Y OIRECTION (m)

DISPERSIVITY Z DIRECTION (m) $1.00 E+00$

PORE VELOCITY $(\mathrm{m} / Y) \quad 6.25 E+21$

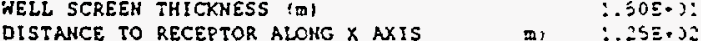

$\begin{array}{lll}\text { DISTANCE TO RECEPTOR ALONG X AXIS } & \text { m, } & 325 \equiv+32 \\ \text { DISTAHCE TO RECEPTOR ALONG Y AXIS } & \text { (m, } 30 E+10\end{array}$

OISTANCE TO RECEPTOR ALONG 2 AXIS mI $3.30 E+30$

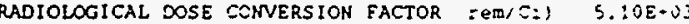

UNITS OF CONTAMINANT

LIMITING SOIL CONCENTRATION CAIOUITTIOH

$\gg$ INITIAL ACTIVITY CONVBRTED TO MASS (mq) B.31E+03

$\gg$ VALUES CALCULATED IN SOURCE SUBRCUTINE

......

LEACH RATE CONSTANT $(1 / Y) \quad 1.2081 E-01$

UNSATURATED PORE VELOCITY (m/Y) $5.8000 E+00$

DECAY CONSTANT (S) $(1 / Y)$

RETARDATION FACTOR(S) (SATURATED) 2.2010E+03

RETARDATION FACTOR (UNSATURATSD)

SOWBILITY LIMITED MASS (mg) $5.6458 E+15$

SOWBILITY LIMITED ACTIVITY (Ci) $\quad: .0745 E+12$

TRAHSIT TIME IN UHSAT ZOHE (YeACS) $\quad: .724: E-25$

FRACTICH DECAYED DURING UNSAT TRANSPORT $5.9754 \mathrm{E}-:$ :)

COMMITTED EFFECTIVE DOSE EQU:VALENT ZALZUAT:DH

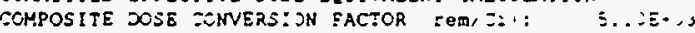

IIMIT!NG TRCUNDWATER ECNCENTRAT:CH $G: 21:$ :

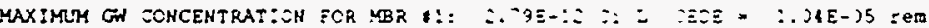

MAXIMUM CECE 1. 34 B-JS =E

PEAK TIME $(y)$ : $\quad 2.4-650: 3+;$

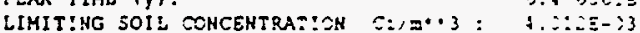

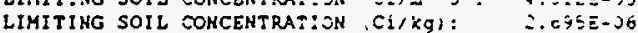

LIMITING INVENTORY IN SOIL (Ci):

LIAITING :NVEHTORY IN SOIL Dgl:

SPECIFIC ACTIVITY (C1/g): $1.903 E-01$
EXECUTION TIME (seCONds)

TIME OF RUN 10:11:05.2

DATE OF RUM $11 / 05 / 96$

$\gg$ PITLE OF PROJECT

$\mathrm{Hi}-5 \mathrm{i}$

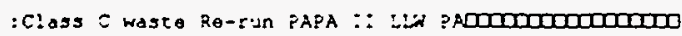

GAUSSIAN ZUADRATURE SOLT:ON

GAUSSIAN ZUADRA

IMODE: 1

KFLAG: 1 (0) CONC VS TIME: (1, PEAK CCNC ANE LIMITIMG SOIL COHC

IMODEL: 1 (1) SURF OR BURIED SOURCE: 2) POND SOURCE: (31 TABUIATED SOURCE FUNCTION

ITYPE: 0 10, 'JERT AVG; (1) NON-VERT AVG; 21 AVG FRCM ; :O 20

$\gg$ INPUT DATA

MUMBE⿱一⿻上丨.

MUMBER OF RADIOACTIVE PROGENY

$\begin{array}{ll}\text { LENGTH OF SOURCE ?ARALLEL TO GN FLOW (IM) } & 4.30 E+01 \\ \text { HIDTH OF SOURCE PERPENDICULAR TO GH FLOW (m) } & 2.40 E+01\end{array}$

了 


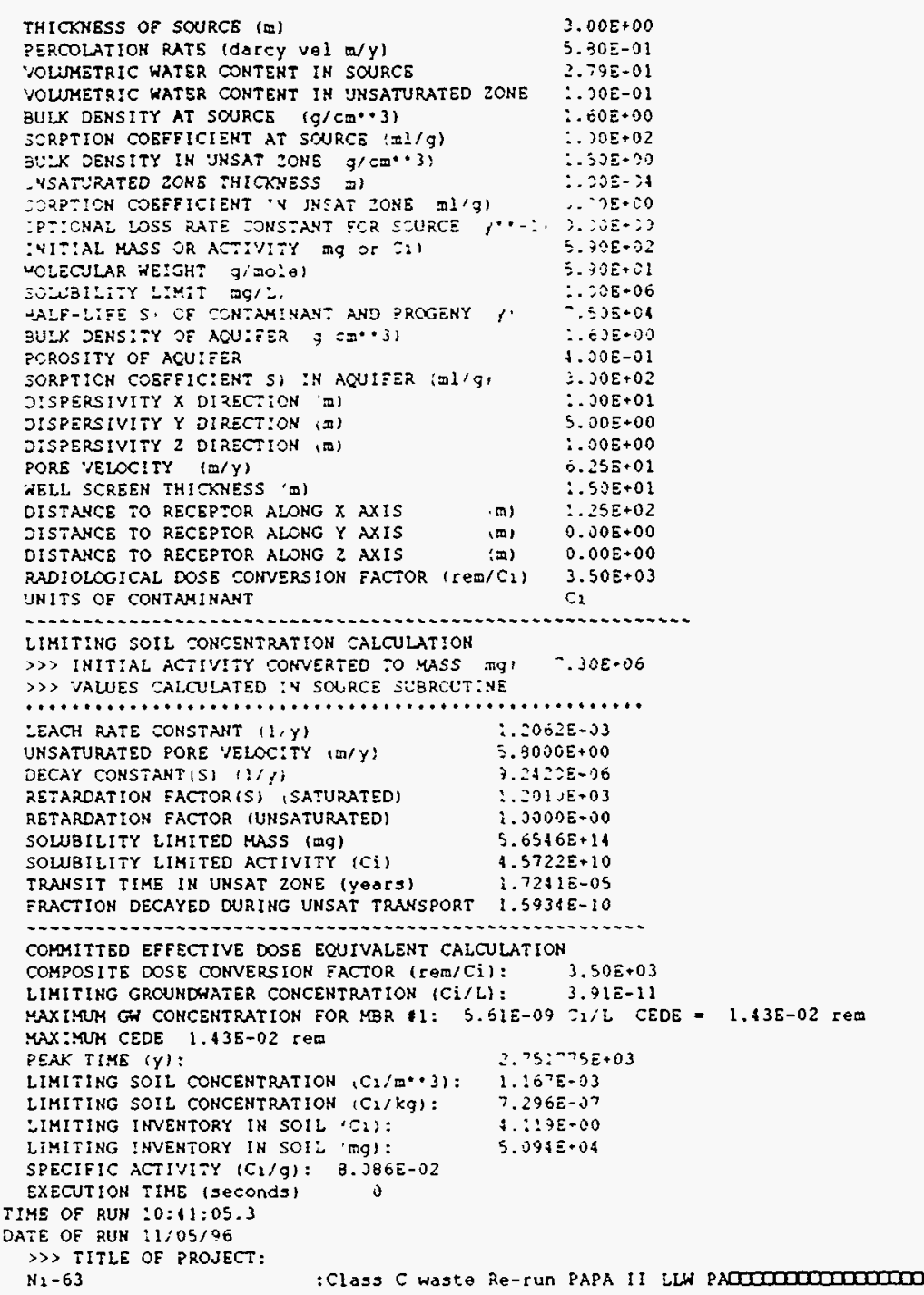

GAUSSIAN QUADRATURE SOLUTION

MODEL OPTIONS

IMODE: 1

KFLAG: 1 10,CONC VS TIMS: (1)PSAK EONC AND LIM:TING SOIL CONC

IKODEL: 1 (:) SURF OR BURIED SOURCE; (2) POND SZURCE: (3) TABULATED SOLRCE FUNC:ICN

ITYPE:0 (0) VERT AVG; (1) NCN-VERT AVG: 2) AVG FRCM O TO 20

\INPUT DATA

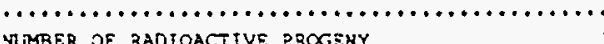

LENGTH OF SCURCE PARALEE: TO GN ELOH MI TI T. JOE+0

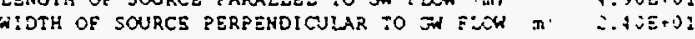

TH:CKNESS OF SOURCE (II)

PERCOLATION RATE : DATCY VEI IIYY

YOUMETRIC WATER CONTENT IN SOLRCE

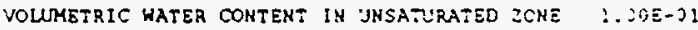

$\begin{array}{ll}\text { BULK DENSITY AT SOURCE } g / \mathrm{cm} \cdots 31 & \vdots .00 \mathrm{3}+00 \\ \text { SORPTION COEFFICIBNT AT SOURCE 'ml/g) } & \vdots .00 \mathrm{0}+02\end{array}$

SORPTION COEFFICIBNT AT SOLRCE 'ml/g)

BULK DENSITY IN UNSAT ZONE $\left(g / \mathrm{CM}^{\circ} 3\right) \quad \vdots 30 E+00$

UHSATURATED ZONS THICKNESS (m)

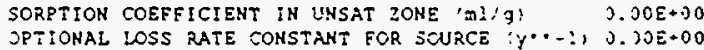

INITIAL MASS OR ACTIVITY II OF Ci)

MOLECJLAR WE:GHT (g/EOLO) D. $30 E+0$

SOUBILITY L:MIT (mg/L)

HALF-LIFE:SI JE CONTAMINANT AND PROGENY Y

BULK DENSITY OF AQUIFER $(\mathrm{g} / \mathrm{cm} * 3) \quad \vdots 50 \mathrm{c}+30$

POROSITY OF AQUIFER 1. OOE-O

SORPTION COEFFICIENT(S) IN ACUIFER $(\mathrm{ml} / \mathrm{g})$ 3.00E+02

$\begin{array}{ll}\text { DISPERSIVITY X DIRECT:ON }(\mathrm{m}) & 500 E+01 \\ \text { JISEERSIVITY Y OIRECTION }(\mathrm{m}) & 5.30 \mathrm{E}+00\end{array}$

$\begin{array}{ll}\text { DISPERSIVITY Y OIRECTION (m) } & 5.30 E+00 \\ \text { DISPERSIVITY Z DIRECTION (m) } & 1.005+00\end{array}$

PORE VELOCITY (ID/Y)

WELL SCREEN THICKNESS (m) i.5OE+0

DISTANCE TO RECEPTOR ALONG $X$ AXIS in $1.25 E+02$ 


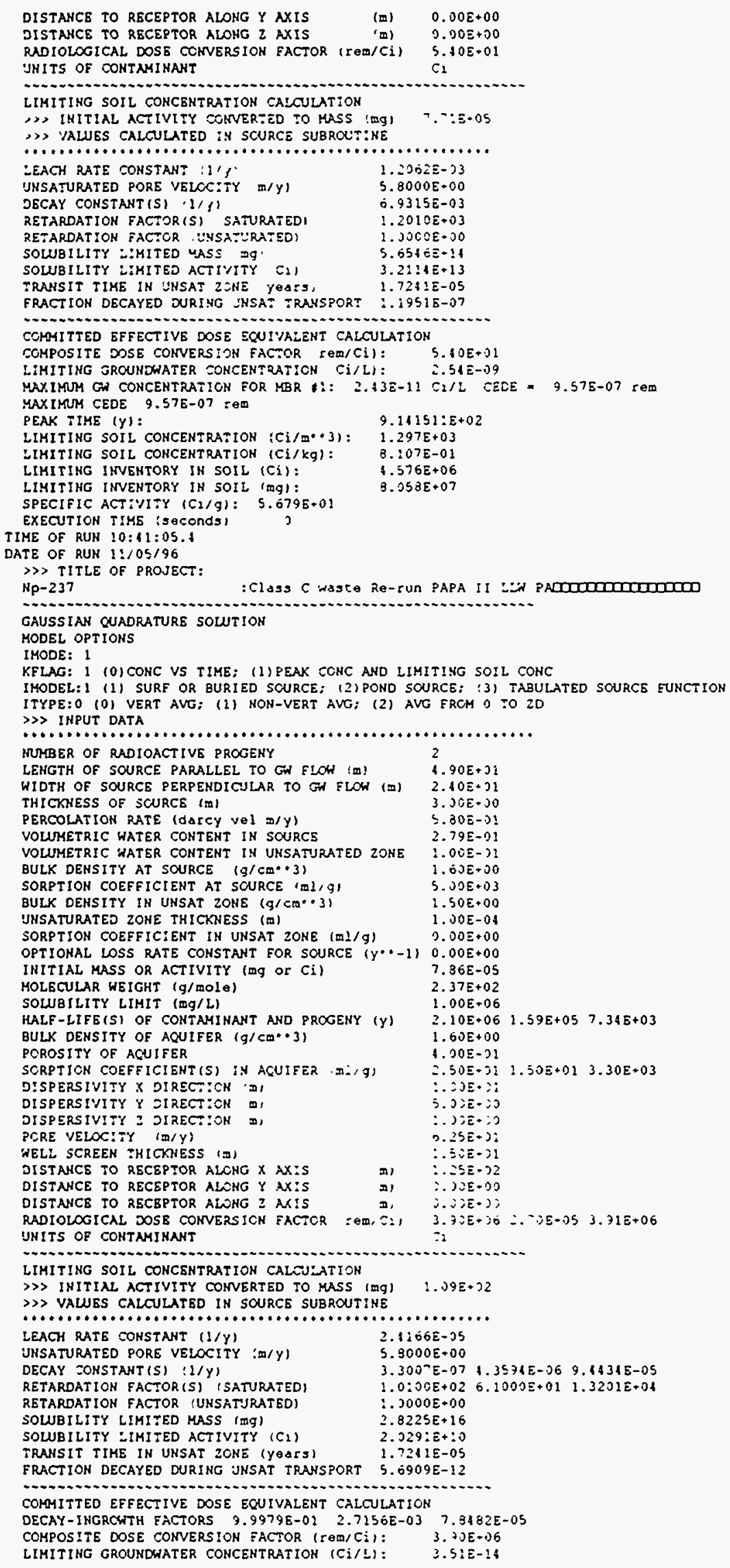


NOTE: PROGENY CONCENTRATIOHS ARE REPORTED AT THE TIME OF THE MAXTMUM PARENT CONCENTRATICN HAXIMAM GN COHCENTRATION FOR MBR $11: 5.46 E-17$ : 1 / CESE = $1.55 \mathrm{i}-07$ reW HAXIMUM GN COMCENTRATION FOR MBR 12: 2.45E-: 21,1 EECE $=1.34 \mathrm{E}-11$ rem YAX:MUM JT CONCEMTRATION FOR MBR $3: 3.28 E-23$ C2/2 EEDE $=9.35 E-14$ rem YAX:MUM $Z E D E$ I.55E-07 reD

PENK T:KE $(Y)$ :

PEAK T:KE (Y):

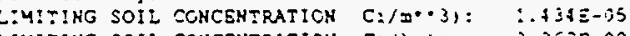

ZM:TIYG SOIL CONCENTRATION, $C: / \mathrm{kgl:} \quad 3.76: E-79$

EMTT:NG INVENTORY IN SOIL (CI): $5.35 \times \bar{E}-1,2$

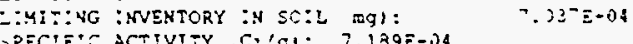

SPECIF:Z ACTIVITY C C:/GI: $7.289 \mathrm{E}-34$

EXECUT:ON TIME , Soconas!

T:YE JE RUN $10: 11: 05$.

SATE OE $2 \cup N::, 95,96$

, T:ZEE JE PROJECT:

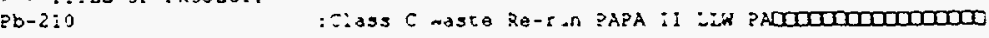

GAUSSIAN TUADRATURE SOUTION

GAUSSIAN TUADRA OPT:OKS

IHOOE: 1

KFLAG: : 0, CONC VS TIME: 11 PEAK CONC AND ZIMITING SO:L CONC

IMCDE::! 1: SURE OR BUR:SD SOLRCE; '2: PCND SOURCE; '3; :ABULATED SOURCE FUNCT:CN

:TYPE:0 (0) VERT AVG; 111 NON-VERT AVG; , 21 AVG EROM 0 TO 20

$\gg$ INPUT DATA

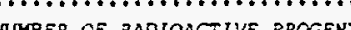

DTOACIVE PROGENY

PARALLEL TO GI FLOW 'MI

WIOTH OF SOURCE PERPENDICULAR TO GN FLOW (m) $2.40 E+01$

THIOWESS OF SOURCE (m)

PERCOLATICN RATE IdATCY vel $a / Y$;

VOLUMETRIC WATSR SCNTENT IN SOLFCE

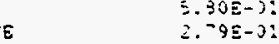

IN UNSATURATED ZCNE $\because 3 O E-J i$

BULK DENSITY AT SCURCE (G/CRM3)

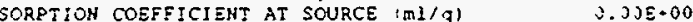

BULK DEHSITY IN UESAT ZONE $\left(9 / \mathrm{CT}^{\prime} \cdot 3\right)$

UNSATURATED ZONE THICKNESS III

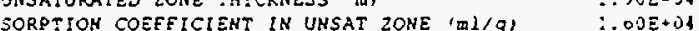

OPTIONAL LOSS RATE CONSTANT FOR SOURCE, . - -1, $0.005 \cdot 00$

OPTIONAL LOSS FATE CONSTANT FOR SOURCE Y Y $\cdots-110.30 E+00$

INITIAL MASS OR ACTIVITY img Or C2) $\quad 2.00 E-1) 1$

$\begin{array}{ll}\text { MOLECULAR WEIGHT ' } g / \text { mOLO) } & 2.10 E+02 \\ \text { SOUBILITY LIMIT (mg/L) } & 1.00 E+06\end{array}$

SOUBILITY LIMIT (mg/L)
HALE-LIFE (S) OF CONTAMINANT AND PROGENY (y) $1.00 E+06$
$2.23 E+01$

HALE-LIFE(S) OF CONTAMINANT AND PROGENY (y) $2.23 E+01$

POROSITY OF AQUIFER

SORPTION COSFFICIENT(S) IN AQUIFER $(\mathrm{m} 1 / \mathrm{g})$

DISPERSIVITY X DIRECTION (to) $1.00 E+01$

DISPERSIVITY Y DIRECTION (a) $5.00 E+00$

DISPERSIVITY 2 OIRECTION (m)

PORE VELOCITY $(\mathrm{m} / Y)$ (D)

WELL SCREEN THICKNESS (m)

DISTANCE TO RECEPTOR ALONG $X$ AXIS (a) $\quad: 25 E+02$

DISTANCE TO RECEPTOR ALONG Y AXIS (m) $3.00 \varepsilon+00$

OISTANCE TO RECEPTOR ALONG $z$ AXIS 'mi $0.00 E+00$

RADIOLOGICAL DOSE CONVERSION EACTOR, rem/C1I D. $70 E+06$

UN:TS OF CONTAMINANT

$\mathrm{C}_{2}$

LIMITING SOIL CONCENTRATION CALCULATION

$\gg$ INITIAL ACTIVITY CONVERTED TO MASS (mg) $2.62 \mathrm{E}+00$

\> VALUES CALCULATED IN SOURCE SUBROUTINE

UNSATURATED PORE VELOCITY (m/Y) $5.8000 E+00$

DECAY CONSTANT (S) ' $1 / Y$ Y

RETARDATION FACTOR(S) ISATURATED)

RETARDATICH FACTCR UNSATURATEDI

JOUUBILITY OMITED UASS MOI

SOLUDILTY DUITEO ACTIVTY CIT

TRANSIT TIME IH JNSAT ZONE (YOATS)

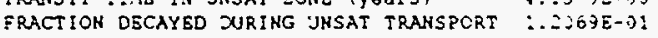

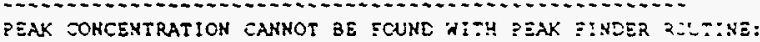

JSE CONCENTRATION 'US TIME MCLE :O JETERM:NE ?EAK

NO SOIL CCNCENTRAT!ON LIM:TS GALOULATED S:ACE THE

AVERAGE INTEGRATED COHCENTRATICN IS ZERO

TIME OF RUN 10:41:OS.

DATE OF RUN $11 / 05 / 96$

$\gg$ TITLE OF PROTECT:

$\mathrm{Pu}-238$

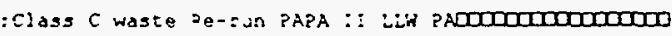

GAUSSIAN CUACRATURE SOUTION

MODEL OET:ONS

IYCDE: ?

KFLAG: : MCONC IS T:ME: 11PSAK CONC AND ZIHIT!NG SO:L TONC

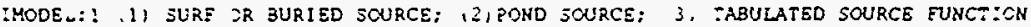

ITYPE:0 (0) VERT AVE: (1) NON-VERT AVG: 2) AVG FRCM 0 TO 20

$\gg$ INPUT DATA

MTMER OE RADTOACTIVE PROGEMY

LENGTH OF SOURCE PARALLEL TO GN FLON (m) $1.90 \mathrm{~m}+1$

WIDTH OF SOURCE RERPENDICULAR TO GW F:OW (a) $2,120+01$

THICKNESS OF SOURCE (a)

PERCOLATION RATE idaTCY vol $\mathrm{m} / \mathrm{y}$ I

VOUMETRIC WATER CONTENT IN SOURCE $2.79 \mathrm{E}-0$ ! 


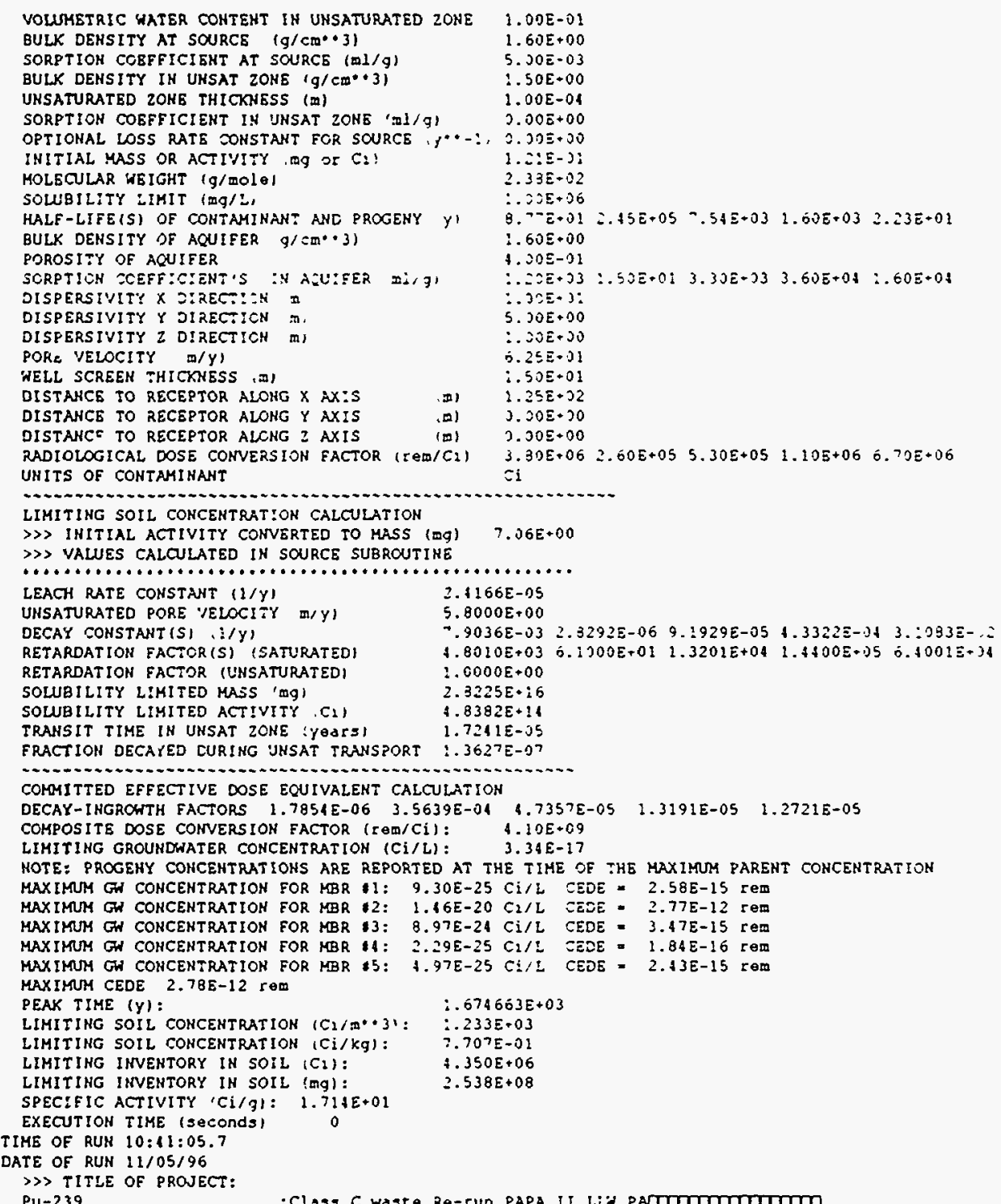




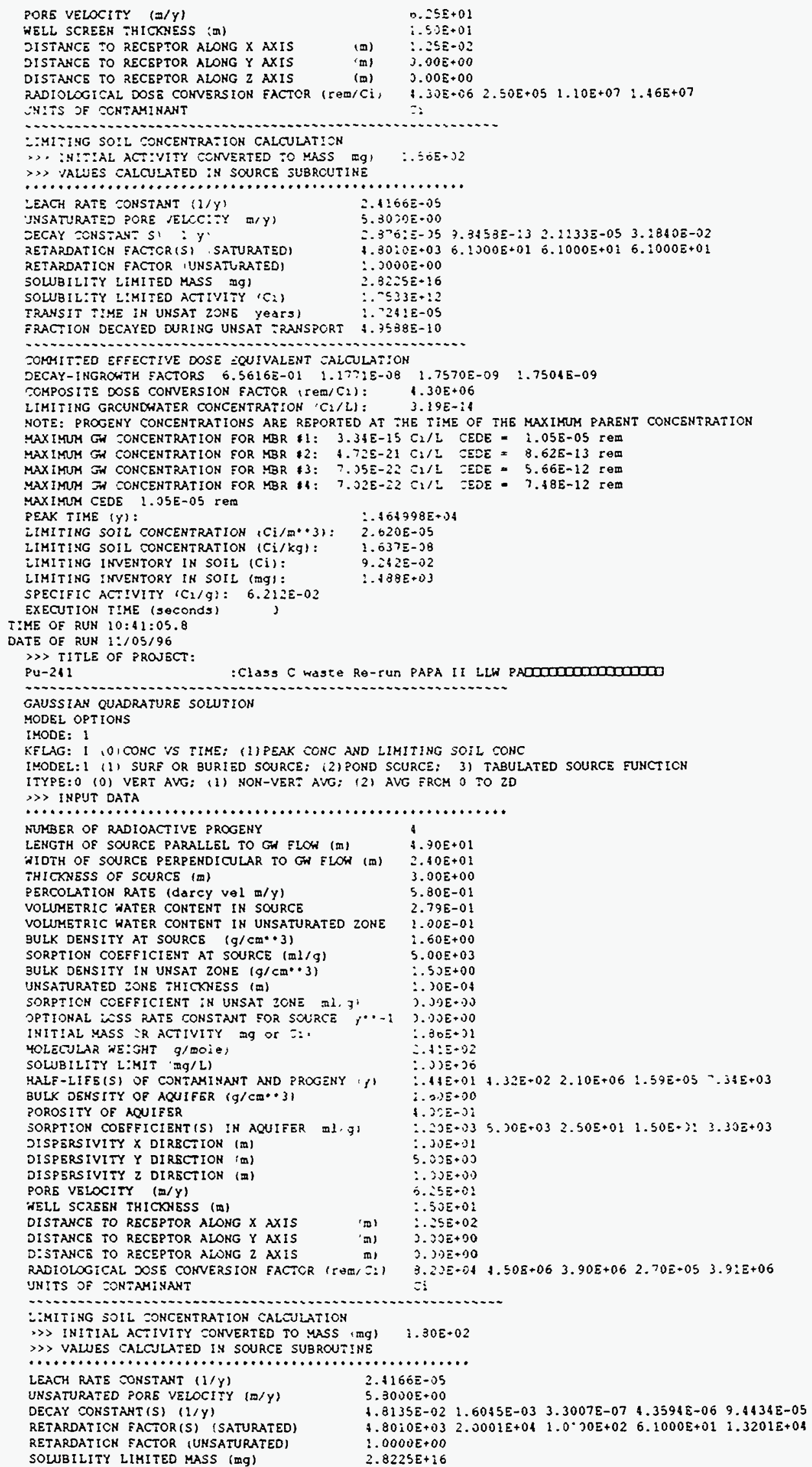




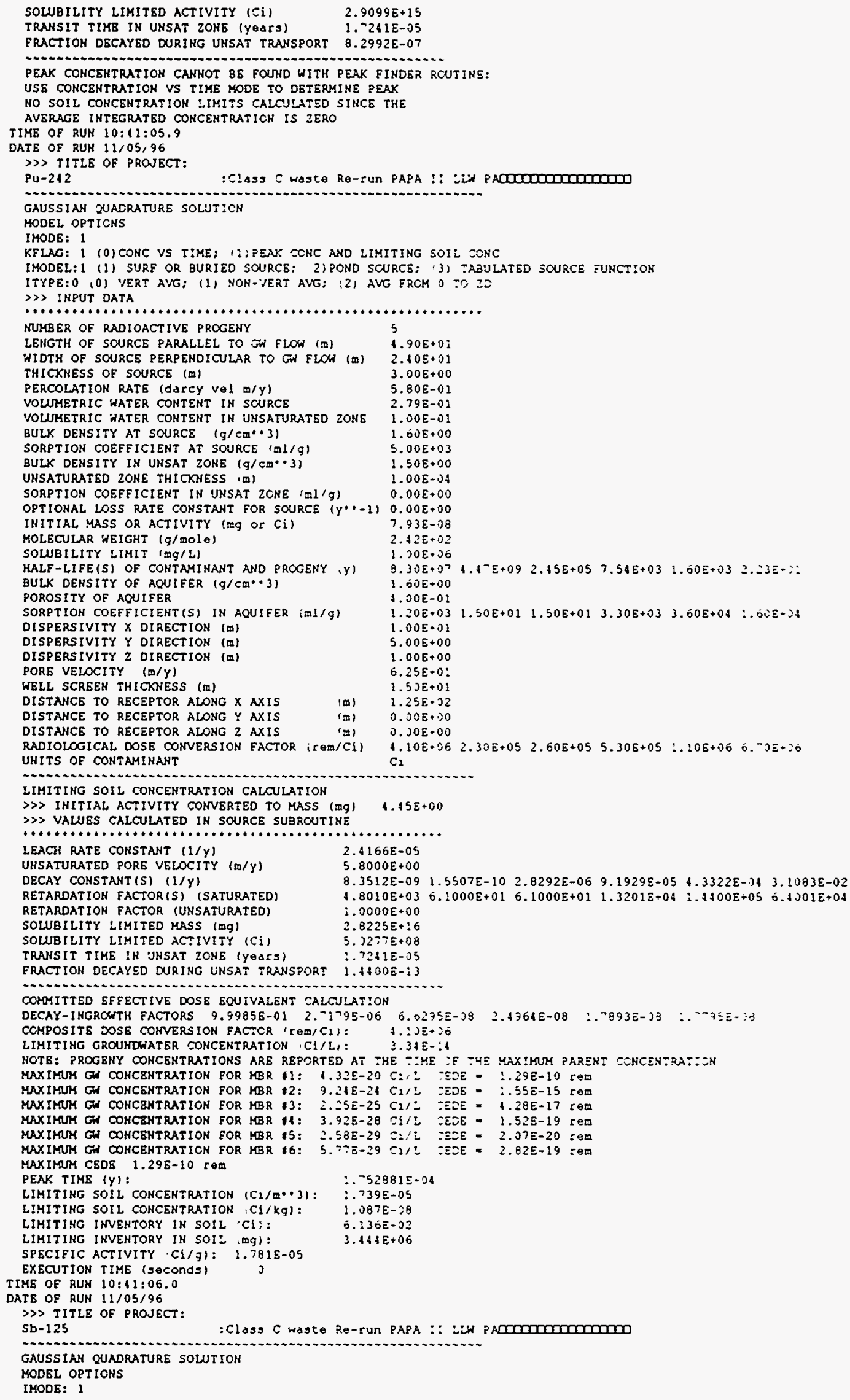


KELAG: 1 (0) CONC VS TIME: (1) PEAK CONC AND LIMIIING SOIL CONC

IMODEL: I (1) SURE OR BURIEO SOURCE; (2) POND SOURCE; 3, TABULATED SOURCE FUNCTION :TYPE:0 (0) VERT AVG: (1) NON-VERT AVG: (2) AVG ERCM 3 TO $2 D$ $\gg$ INPUT DATA

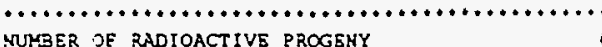
LENGT4 OF SCURCE PARALLEL TO GN ELAA (D) WIDTH OF SOURCE PBRPENDICULAR TO GN ELA THICKNESS OF SOURCE $\mathrm{fm}$

PERCOLATION RATE (dATCY VOl m/Y)

YCUMETRIC WATER CONTENT IN SOURCE

WOUMETRIC WATER CONTSHT IH UNSATURATED ZOHE

BULK DENSITY AT SOURCE $(\mathrm{g} / \mathrm{CM} \cdots 3)$

SORPT: TH TOEFETCTENT AT SOURCE

SULK JENS:

UNSATURATED ZONE THICKNESS (IN)

OPT:CNAL LOSS RATS CONSTANT FOR SOURCE,$\gamma^{*+-1)} 0.00 \mathrm{E}+00$

:NITIAL MASS OR ACTIVITY ( $m$ or $C i$ ) $3.55 E+02$

MOLECULAR WE:GHT $(\mathrm{g} / \mathrm{mOl}$ (e)

SOWBILITY : :MIT (mG/L)

HALF-LIES(S) OF CONTAMINANT AND PROGENY Y) 2 ' $3 E+00$

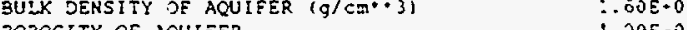

POROSITY OF ACUIEER

SORPTION COEFFICIENT(S) IN AQUIEER (ml/g) $1.50 E+02$

DISPERSIVITY $X$ PIRECTION (D) $1.00 E+01$

DISPERSIVITY Y DIRECTION (⿴囗十) $5.00 E+00$

DISPERSIVITY $Z$ SIRECTION (⿴囗十) $1.00 E+00$

$0.25 \Xi+01$

WELL SCREEN THICKNESS (D)

DISTANCE TO RECEPTOR ALONG $X$ AXIS

DISTANCE TO RECEPTOR ALONG Y AXIS A) $0.00 E+00$

DISTANCE TO RECEPTOR ALONG $Z$ AXIS (m), $0.00 E+00$

RADIOLOGICAL DOSE CONVERSION FACTOR ( $\left.\mathrm{rem} / \mathrm{C}_{1}\right) \quad 2.60 \mathrm{f}+0$

UNITS OF CONTAMINANT

LiMITING SOIL CONCENTRATION CALCULATION

\> INITIAL ACTIVITY CONVERTEO TO MASS (mg) $3.39 E+02$

\> VALUES CALCULATED IN SOURCE SUBROUTINE

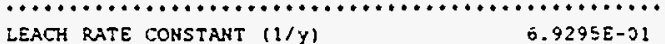

UNSATURATED PORE VELOCITY (II/Y) $5.3000 E+00$

DECAY CONSTANT(S) (1/Y)

RETARDATION FACTOR(S) (SATURATED) O.J100E+02

i. $2000 \mathrm{0}+00$

SOWBILITY LIMITED MASS (mg) 9.2431E+1

SOLUILITY EIMITED ACTIVITY (C2) $1.0320 \mathrm{C} \cdot 12$

TRANSIT TIME IN UNSAT ZONE (Yearsi $\quad .724: 2-05$

ERACTION DECAYED DURING UNSAT TRANSPORT $\$ .3776 \mathrm{~F}-106$

COMGITTED EFFECTIVE DOSE EQUIVALENT CALOULATION

COMPOSITE DOSE CONVERSION EACTOR (rem/C1): $2.60 E+03$

LIMITING GROUNDWATER CONCENTRATIOH $(\mathrm{Ci} / \mathrm{L}): \quad 5.27 \mathrm{E}-11$

MAXIMUM GN CONCENTRATION FOR MBR 1: $9.22 E-29$ C2/L CEDE $=1.75 E-22$ rem

MAXIMUM CEDE $1.75 E-22$ rex

PEAK TIME $(y)$

LIMITING SOIL CONCENTRATIOH (Ci/m.03I:

LIMITING SOIL CONCBNTRATION $(\mathrm{Ci} / \mathrm{m} \cdots 3): 5.7545+16$

LIMITING INVENTORY IN SOIL (Ci): $\quad 2.030 E+20$

SPECIFIC ACTIVITY (C:I/q): $1.048 \mathrm{~B}+03$

NOTE: THE LIMITING SOIL CONCENTRATION OF $3.596 \mathrm{E}+10 \approx 1 / 9$

EXCEEDS THE SPECIEIC ACTIV:TY OF T4E 4 UC: :ZE.

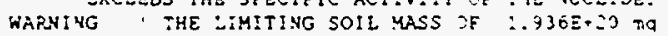

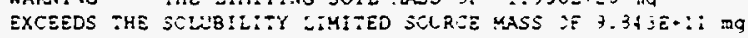

EXECUT:ON :ZME seconds,

T:ME OF RUN :0:41:06.3

DAT5 DF REN $11,05 / 96$

\> T:TLE OF PROJECT:

Sr -90

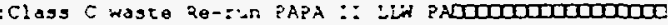

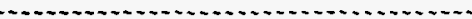

GAUSSIAK SUADRATIRE SOLUTIOH

MODEL OPTICHS

IMODB: 1

KELAG: : (0)CONC VS TIMB: (1) PEAK CONC AND ZIMITING SOI $\mathrm{L}$ LCNC

:MODEL: 1 (1) SURF OR BURIED SOURCE: (2) POND SOURCE; (3) TABULATSD SOURCE FUNCT:CN

ITYPE:0 (0) VERT AVG: (1) NON-VERT AVG: (2) AVG FROM O TO ZD

$\gg$ INPUT DATA

U.

EENGTH JF SOURCE PARALLEL TO GN FLON (m)

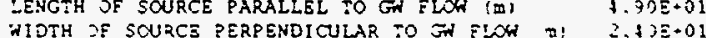

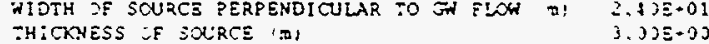

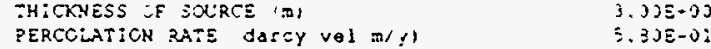

VOUHETRIC WATER CONTENT ON SOLRCE

VOLUMETRIC AATER CCNTENT IH UNSATURATED ZONE $: .20 E-02$

BULK ZENSITY AT SOURCE $G / C D \cdots 31 \quad: .00=00$

SORPTICN COEFE?CIENT AT SOURCS ML/g)

BULK DENSITY IN UNSAT ZONE $(g / \mathrm{CM} \cdots 3) \quad 3.50 E+00$

UNSATURATED ZONE TH:CKNESS $(\mathrm{m}) \quad$.JOE-06

SORPTION COEFFICIENT IN UNSAT ZONE (m!/g) $0.00 E+00$

OPTIONAL LOSS RATE CONSTANT FOR SCURCE ( $y-1) 0.00 E+00$

INITIAL MASS OR ACTIVITY (Dg OI Ci) $1.30 \mathrm{E}+00$ 


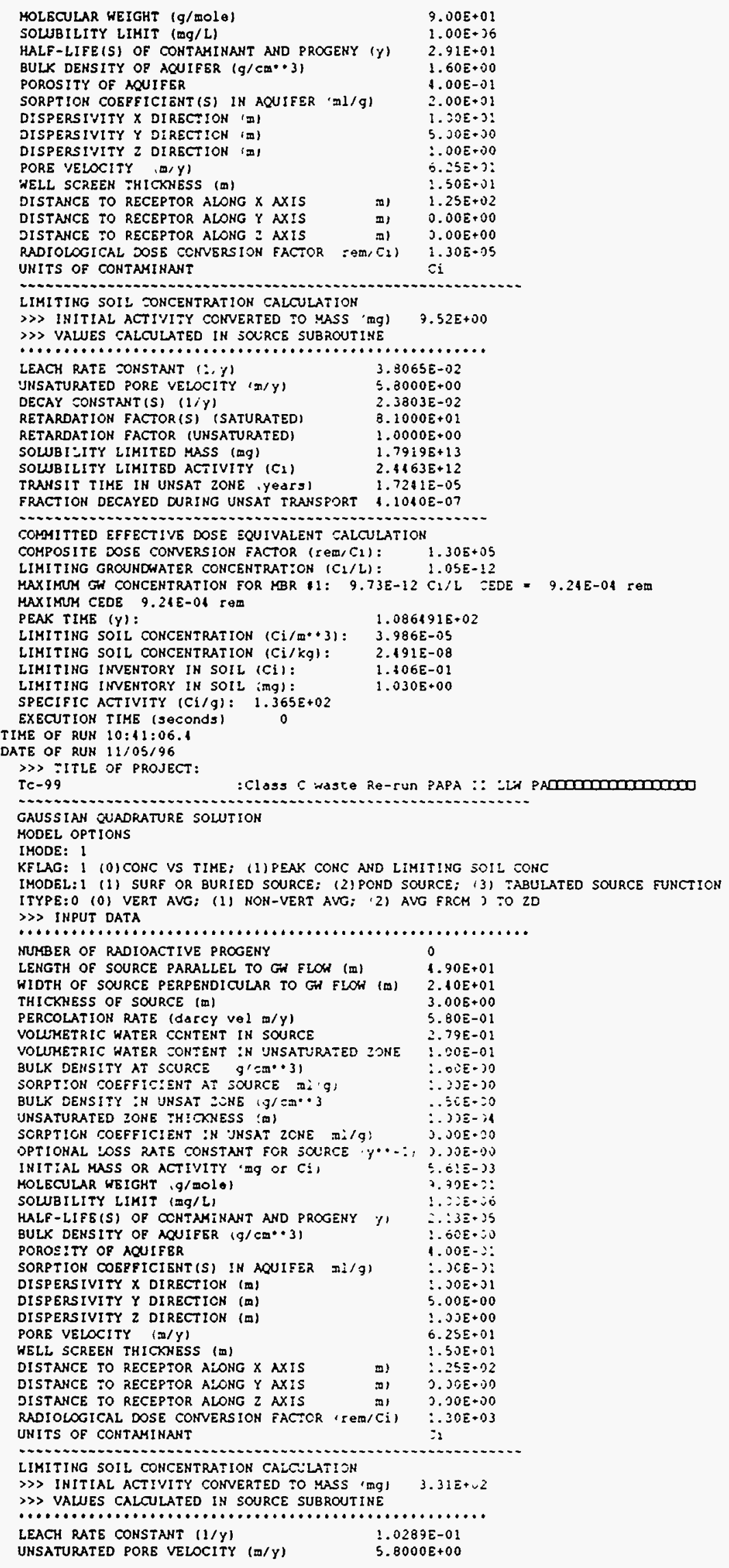


DECAY CONSTANT (S) $(1 / Y)$

RETARDATION EACTOR (S) (SATURATED) $\quad: .10008+00$

RETARDATION FACTOR (UNSATURATED)

SOLUB!LITY LIMITED MASS (mg) $\quad 0.0291 \mathrm{E}+12$

SOUUBIEITY LIMITED ACTIVITY (Ci)

TRANSIT TIHE IN UNSAT ZONE (YOAIS)

FRACTION DBCAYSD DURING UNSAT TRANSPORT S.6:? P'E-1:

COMITTSD SFFECTIVS NCSE SCUIVALENT CALCJLATION

COMPOSITS DOSE CONVERSION EACTOR IOE/C2,: :.32E.0

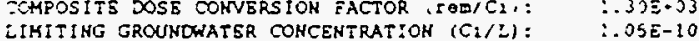

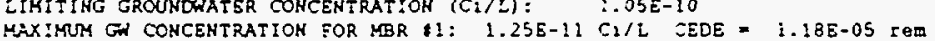

MAXIMUM GA CONCENTRATION FOR MBR $11:$ 1.25E-11 EI/L
MAXIMM CEOS $1.18 E-05$ rem
PEAK

PEAK TIME $(y)$

GIMIT:ING SOIL CONCENTRAT:CN $C_{2}$ m+31: $\quad: 345 E-25$

G̈MITING SOIL CONCENTRATION (CI/KG):

LIMIT:NG :NVENTORY IN SOIL $\left(\mathrm{C}_{2}\right)$ :

EIMITING INVEMTORY IN SOIL RQ):

SPECIFIC ACTIVITY $\left\{C_{1 / 3}\right): \quad: 697 E-02$

EXECUT:ON TIME (seconds)

$2.776 E+03$ 